\title{
SALIENT Keypoint DETECTORS AND COMPACT FEATURE DESCRIPTORS FOR 3D PERCEPTION
}

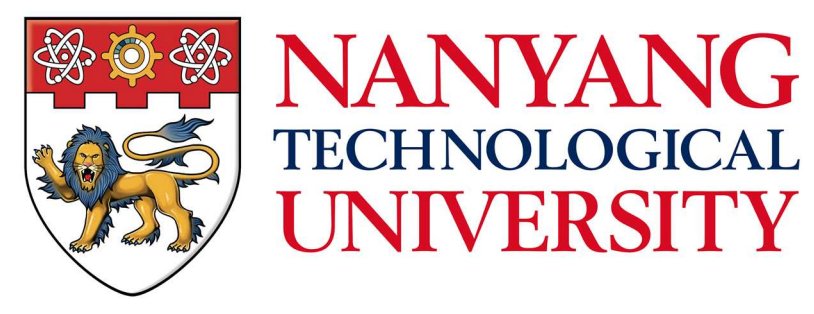

\author{
A thesis submitted to \\ the School of Computer Science \& Engineering \\ Nanyang Technological University
}

by

Sai Manoj Prakhya

in fulfillment of the requirements

for the degree of Doctor of Philosophy

December 2016 


\section{Acknowledgements}

At the end of my undergraduate studies, I had two choices, one is to take up a job as a software engineer, while the other is to pursue PhD at SCSE, NTU, Singapore. I chose to pursue doctoral studies at NTU. One of the most successful Indian businessmen, Ratan Tata once said, 'I don't believe in taking right decisions, I take decisions and make them right'. And now, I can say with absolute confidence that I took the decision to pursue $\mathrm{PhD}$ at NTU and made it right.

Behind this confidence lies many people who have meticulously guided and helped me throughout my $\mathrm{PhD}$ life. This journey would not be possible without them. I would like to sincerely thank

Prof. Weisi Lin for accepting me as his $\mathrm{PhD}$ student, allowing me to pursue my research interests and helping me in making right decisions. I still remember the very first discussion we had where he instilled the confidence in me to pursue $\mathrm{PhD}$ in the field of robotics that I am inclined towards. At the end of two years, when I was struggling with my research, he gave his complete time by engaging in long discussions during the weekends or even while travelling in local transport. He helped me focus on a single topic so that the efforts I put in are magnified and can help in producing publishable works. His critical view on writing papers, format and grammar, conducting right experiments and making right claims in the paper have been greatly helpful and I will cherish this knowledge throughout my life. A simple 'Thank you' or for that sake any word or deed can never equate the way he had nurtured me throughout my $\mathrm{PhD}$ life and more.

Dr. Bingbing Liu While Prof. Weisi gave me the freedom to work on robotics, Dr. Bingbing provided personal guidance and resources to make this journey fruitful. I still remember the first conversation that we had while he drove me to A*STAR. He said, "Sai, remember one thing, there is no free lunch in Singapore". His stern actions when I was taking research easily had kept me on toes and pursue higher goals. In the first two years, he made himself available everyday by sitting next to me in the robotics lab at A*STAR. 
That was very much needed and helpful as I was a fresh graduate coming out of university with absolutely no idea. I am fortunate to have a resourceful person to help all the time and get hands-on experience with expensive sensors which are available only at handful places all over the world. I still remember the very first report that Dr. Bingbing helped in correcting the content and grammar for more than an hour. I had to revisit my high school grammar to get things right. Later, his push to only publish at the very best robotic conferences such as ICRA and IROS are the most valued assets that I portray. His support and confidence in me have always made me feel better in tough times.

Dr. Vijay Chandrasekhar and Dr. Jie Lin For being very supportive and helpful in refining my work and pinpointing its direction. They offered in-depth technical understanding and guidance on few topics which helped me churn two publishable works in very short time. Their positive outlook during our interactions has instilled confidence and their extremely critical reviews have aided in conducting right experiments and writing good reports. It has been a great pleasure working with them and I look forward for more.

Dr. Usman Qayyum Though I worked with Usman for a short time, his help in developing my first work is highly commendable. I had complete freedom with him in asking even a silly doubt to engaging in long discussions on what experiment I should perform next.

I2R staff Yan Rui, Yuan Wei, Wu Xiaojun, Albertus, Zhang Qun, Chi Yuan, Wu Pengfei, Shuangli Du, Wang Bin, Xiaoli, Pan Yu, Ben, Tai Wen, Boon Siew, Tee Keng Peng, Anthony, Dilip and Somchaya for engaging in technical discussions, attending my presentations, and for those awesome lunches that we used to go everyday.

Groupmates Sharath Chandra, Vinit, Yang Huan, Yuming, Issac, Yuan Yuan, Yabin, Wentao, Li Qiaohang, Tra Trung Anh, Xiaolong Bai, Xu Long, Manish, Jinjian, Gu and Dong Lu for maintaining extremely conducive environment during the group meetings, help in reviewing the papers and engaging in fruitful discussions.

Lab Staff Ms. Chua Poo Hua and Ms. Ng-Siom Siew Ling for their help in resolving computer issues when my PC broke down and help in printing a poster.

SCSE, NTU for offering financial support and the opportunity to pursue $\mathrm{PhD}$.

My Parents and brother for their unconditional love, support and trust. 


\section{Contents}

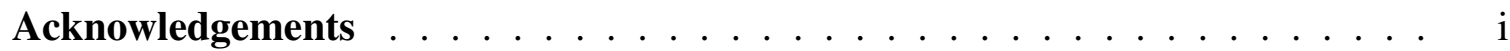

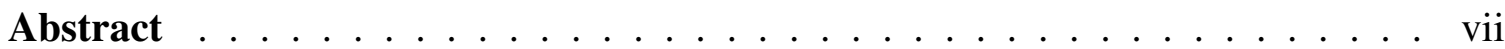

Publications 1

1 Introduction 3

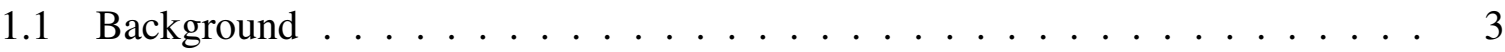

1.2 Challenges in 3D Keypoint Detection . . . . . . . . . . . . . 6

1.2.1 Keypoint Clusters for 3D Object Recognition . . . . . . . . . . . . 6

1.2.2 Distinctive Keypoints for Pose Estimation ～. . . . . . . . . . . . 7

1.2.3 Unreliable Planar Keypoints . . . . . . . . . . . . . . . . . 7

1.3 Limitations of 3D Feature Descriptors . . . . . . . . . . . . . . . . 8

1.4 Thesis Structure: Motivation \& Contributions . . . . . . . . . . . . 8

1.4.1 Salient 3D Keypoint Detection . . . . . . . . . . . . . . . . . . 9

1.4.2 Compact 3D Feature Description . . . . . . . . . . . . . . . . . . 12

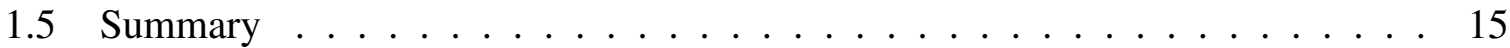

2 Literature Review $\quad 17$

2.1 Modules in 3D Perception Applications . . . . . . . . . . . . . 17

2.2 Related Work . . . . . . . . . . . . . . . . . . . . . . . . . . . . 19

2.3 3D Keypoint Detectors . . . . . . . . . . . . . . . . . . . . . . . 19

2.4 RGB-D Camera based Odometry . . . . . . . . . . . . . . . . . . 21

2.4.1 Sparse methods that use RGB and depth information . . . . . . . . 21

2.4.2 Dense methods that use RGB and depth information . . . . . . . . . 22

2.4.3 Dense methods that use depth information alone . . . . . . . . 22

2.5 3D Feature Description . . . . . . . . . . . . . . . . 23

2.5.1 FPFH: Fast Point Feature Histograms _ . . . . . . . . . . . . 23

2.5.2 SHOT: Signatures of Histograms of OrienTations . . . . . . . . . . . . 24 
2.5.3 RoPS: Rotational Projection Statistics . . . . . . . . . . . . . . 25

2.5.4 Other Real Valued 3D Feature Descriptors _ . . . . . . . . . . 25

3 Histogram of Normal Orientations (HoNO) based Keypoint Detector 28

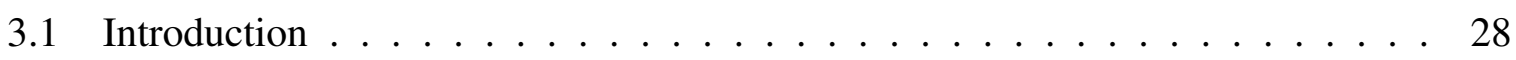

3.2 The Proposed Keypoint Detector . . . . . . . . . . . . . . . . . . . . . . . . . 29

3.2.1 HoNO: Histogram of Normal Orientations . . . . . . . . . . . . . . . 29

3.2 .2 Keypoint Detection . . . . . . . . . . . . . . . . . . . . . 30

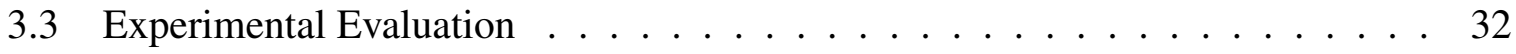

3.3 .1 Datasets . . . . . . . . . . . . . . . . . . 32

3.3.2 Evaluation Metric: Repeatability . . . . . . . . . . . . . 33

3.3 .3 Parameters . . . . . . . . . . . . . . . . . 34

3.3 .4 Repeatability Results . . . . . . . . . . . . . . . . . . 36

3.3.5 Computational Requirements _. . . . . . . . . . . . . 40

3.3.6 HoNO Keypoint sets + SHOT Feature Descriptor . . . . . . . . . . . 41

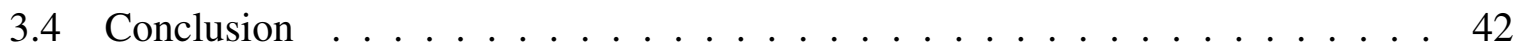

4 Sparse Depth Odometry: Keypoint based Pose Estimation from Dense Depth Data 43

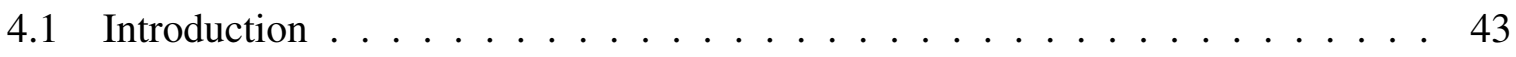

4.2 Sparse Depth Odometry . . . . . . . . . . . . . . . . . . 45

4.2 .1 Voxel Grid Filtering . . . . . . . . . . . . . . . . 46

4.2 .2 Keypoint detection . . . . . . . . . . . . . . . . . 46

4.2.3 Uncertainty Modelling of 3D Keypoints . . . . . . . . . . . . . 46

4.2.4 Frame to Model based Tracking . . . . . . . . . . . . . . . . 47

4.2 .5 Model Update . . . . . . . . . . . . . . . . . . . . . . . . . 48

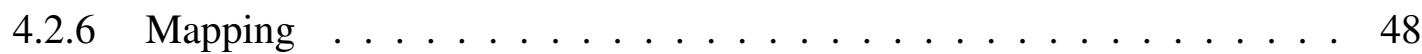

4.3 On Selecting SURE and NARF Keypoint detectors $\ldots \ldots \ldots \ldots \ldots$

4.3 .1 Neighbourhood Variance Test . . . . . . . . . . . . . . . . . . . 49

4.3.2 Neighbourhood Structure Test . . . . . . . . . . . . . . . . . . . 49

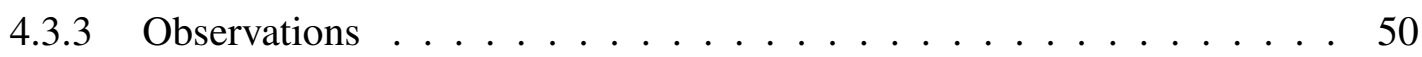

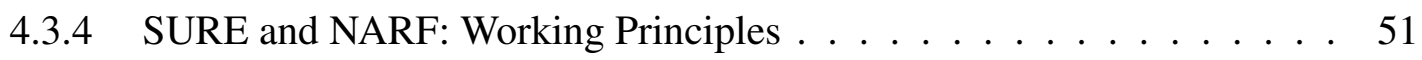

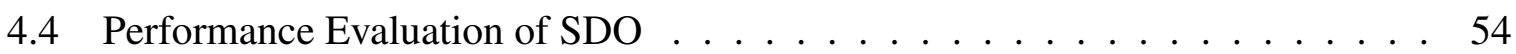

4.4.1 Computational Requirements _. . . . . . . . . . . . . . . . 54

4.4.2 Accuracy and Robustness of Sparse Depth Odometry . . . . . . . . . 56

4.4 .3 3D Mapping with SDO . . . . . . . . . . . . . . . . . . 59

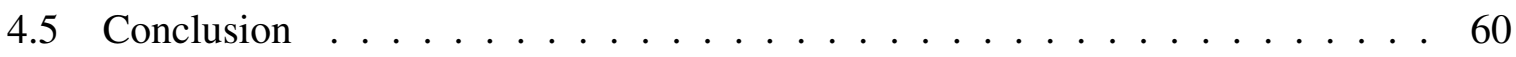


5 3DHoPD: A Fast Low Dimensional Real-valued 3D Feature Descriptor

5.1 Introduction . . . . . . . . . . . . . . . . . 62

5.2 3DHoPD: The Proposed 3D Feature Descriptor . . . . . . . . . . . . . . . 64

5.2.1 Construction ........................ 64

5.2 .2 Matching .......................... 68

5.3 Experimental Evaluation $\ldots \ldots \ldots \ldots$

5.3.1 Evaluation of the 3D Keypoint Transformation Technique . . . . . . . 68

5.3.2 3DHoPD Descriptor Evaluation . . . . . . . . . . . . . 71

5.4 Observations and Discussions $\ldots \ldots \ldots \ldots \ldots$

5.5 Conclusion . . . . . . . . . . . . . . . . . 79

6 B-SHOT: A Binary 3D Feature Descriptor 8

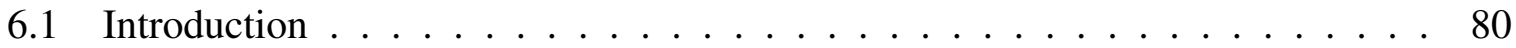

6.2 Related Work . . . . . . . . . . . . . . . . . . . . . . 81

6.2 .1 2D Binary Feature Descriptors . . . . . . . . . . . . . . 82

6.3 The Proposed Technique to Binarize SHOT . . . . . . . . . . . . . . . 83

6.4 Experimental Evaluation . . . . . . . . . . . . . . . . . . . 88

6.4 .1 Experimental Setup . . . . . . . . . . . . . . . . . 88

6.4.2 On Selecting B-SHOT over B-RoPS and B-FPFH . . . . . . . . . . . . 89

6.4.3 Robustifying Recognition Rate for 3D Descriptor Comparison . . . . 94

6.4.4 Comparison of B-SHOT with SHOT, RoPS and FPFH . . . . . . . . 98

6.5 Parameter Evaluation . . . . . . . . . . . . . . . . . . . . . 102

6.6 Computational \& Memory Requirements . . . . . . . . . . . . . . 103

6.6.1 Comparing the Computational Requirements of B-SHOT \& SHOT . . . 104

6.7 Conclusion . . . . . . . . . . . . . . . . . . 106

7 Low Bit-rate 3D Feature Descriptors with Lattice Quantization 107

7.1 Introduction . . . . . . . . . . . . . . . . . . . . . 107

7.2 Related Work . . . . . . . . . . . . . . . . . . . . 108

7.2.1 Compression Techniques in 2D Domain . . . . . . . . . . . . . . . 109

7.2.2 Compressed 3D Feature Descriptors . . . . . . . . . . . . . . 110

7.3 Lattice Quantization . . . . . . . . . . . . . . . . . 111

7.3.1 Lattice Quantization to Compress 3D Feature Descriptors . . . . . . 111

7.3.2 Compressed Domain Matching . . . . . . . . . . . . . . . 114 
7.3.3 Double Stage Lattice Quantization . . . . . . . . . . . . . . . . . 115

7.4 Experimental Setup . . . . . . . . . . . . . . . . . . . 117

7.4.1 Evaluation Methodology . . . . . . . . . . . . . . . . 117

7.5 Compressing Various 3D Feature Descriptors . . . . . . . . . . . . 118

7.5.1 Compressing SHOT Feature Descriptor . . . . . . . . . . . . . 118

7.5.2 Compressing RoPS Feature Descriptor . . . . . . . . . . . . . . 122

7.5.3 Compressing FPFH Feature Descriptor . . . . . . . . . . . . . . . . . 124

7.5.4 Comparing the Compressed Variants of SHOT, RoPS and FPFH . . . 125

7.6 Computational Requirements . . . . . . . . . . . . . . . . . . 126

7.7 Conclusion . . . . . . . . . . . . . . . . . . 128

8 Conclusion and Future Work 129

8.1 Conclusion . . . . . . . . . . . . . . . . . . . . . . . 129

8.2 Future Research Directions . . . . . . . . . . . . . . . . . . . . 131

8.2.1 3D Keypoint Detection: . . . . . . . . . . . . . . . . . . . . 131

8.2.2 RGB-D Odometry Methods: . . . . . . . . . . . . . . . 132

8.2 .3 3D Feature Description: . . . . . . . . . . . . . . . . 133

$\begin{array}{ll}\text { References } & 134\end{array}$ 


\section{Abstract}

3D depth data acquisition has become extremely easy and affordable with the availability of hand-held depth sensors such as Microsoft Kinect, Intel RealSense Camera and Google Tango. Moreover, with the surge in smartphones equipped with depth sensors such as Lenovo Phab2Pro and Asus Zenfone AR, it is quintessential to develop 3D perception applications that are accurate, and run with low memory, computational and bandwidth requirements.

The first two steps of various 3D perception applications, such as Simultaneous Localization and Mapping (SLAM), 3D object recognition, retrieval and 3D reconstruction, are

1. 3D Keypoint Detection - Detect meaningful 3D points of interest that can efficiently represent the input 3D point cloud.

2. 3D Feature Description - Represent the 3D neighbourhood of the detected keypoints with a multi-dimensional vector to determine keypoint correspondences.

The first part of the thesis focuses on 3D keypoint detection, in which, firstly a highly repeatable salient 3D keypoint detection algorithm is proposed. Next, we consider a specific 3D perception application, SLAM with an RGB-D camera, and propose a new 3D keypoint detection module that works best for it. The second part of the thesis focusses on 3D feature description, in which, we firstly propose a fast real valued low dimensional 3D descriptor, then the first binary 3D descriptor in literature and lastly, a set of even lower bitrate 3D descriptors, which are extremely fast to compute, match yet still offer better performance.

Existing 3D keypoint detectors sometimes detect keypoints on non-salient regions/planar regions or, detect noise and glitches as keypoints. In contrary to the existing norm of having distinct keypoints, we propose to detect salient and highly repeatable keypoint sets(groups of keypoints). Towards this, we propose Histogram of Normal Orientations (HoNO) to detect salient regions and effectively remove planar regions by thresholding the kurtosis of HoNO calculated at every point in the point cloud. Then, the final keypoint sets are detected by evaluating the properties of HoNO and neighbourhood covariance matrix. 
Next, we consider a 3D perception problem, SLAM with an RGB-D camera by solely relying on depth data. As a solution, we propose Sparse Depth Odometry (SDO), in which the main contribution lies in the proposal of a new 3D keypoint detection module. The new keypoint detection module comprises of two existing keypoint detectors, SURE and NARF, and is designed based on extensive theoretical and experimental analysis. The proposed keypoint detection module finds reliable keypoints that work well with nearest neighbour association and represent the scene comprehensively while working in real time, which are the key requirements of SDO. SDO powered with the proposed keypoint detection module, estimates the ego-motion of an RGB-D camera solely from its depth data and runs online without a GPU.

As for 3D feature description, existing real valued 3D descriptors are either high dimensional or demand immense computational time for their extraction and matching. Hence we propose 3DHoPD, a new low dimensional 3D feature descriptor that is extremely fast to compute. The novelty lies in compactly encoding the 3D keypoint position by transforming it to a new 3D space, where the keypoints arising from similar 3D surface patches lie close to each other. Then we propose Histograms of Point Distributions (HoPD) descriptor to capture the neighbourhood structure, thus forming 3DHoPD (3D+HoPD). We propose a tailored feature descriptor matching technique, where in, the search space for each keypoint match is reduced by $90 \%$, and then the exact match is found using the proposed HoPD descriptor.

There are several real valued 3D descriptors, but there is no binary 3D descriptor for 3D keypoint matching. Binary descriptors are known for their low memory footprint and fast matching via Hamming distance. Hence, we introduce the first binary 3D descriptor, B-SHOT, by proposing an adaptive binarization technique that converts a real valued vector to a binary vector. We apply this method on a state-of-the-art 3D feature descriptor, SHOT, and create a new binary 3D descriptor. B-SHOT requires 32 times lesser memory for its representation while being 6 times faster in feature descriptor matching, when compared to the SHOT.

Finally, for the applications that require online transfer of 3D descriptors over a network, there is a need to develop compressed 3D descriptors with even lower memory footprint, i.e., bandwidth and yet have high descriptiveness. Therefore, we propose to employ lattice quantization to efficiently compress 3D feature descriptors. These compressed low bitrate 3D descriptors can be directly matched in compressed domain without any need for decompression, hence drastically reducing the memory footprint and computational requirements for matching. We also propose double stage lattice quantization to achieve even more compression in the case of SHOT descriptor. We provide a spectrum of possible bitrates and achievable keypoint matching performance for three state-of-the-art 3D feature descriptors, so that it can aid users to choose the apt one based on the memory, bandwidth and performance requirements. 


\section{Publications List}

\section{Journals}

1. S. M. Prakhya, B. Liu, W. Lin, "Detecting Keypoint Sets on 3D Point Clouds via Histogram of Normal Orientations", Pattern Recognition Letters, Volume 83, Part 1, 1 November 2016, Pages 42-48, ISSN 0167-8655.

- Contributes to Chapter 3: HoNO based Keypoint Detection.

2. S. M. Prakhya, J. Lin, V. Chandrasekhar, W. Lin, B. Liu, “3DHoPD: A Fast Low Dimensional 3D Descriptor”, IEEE Robotics and Automation Letters, Jan 2017.

- Contributes to Chapter 5: 3DHoPD - A Fast Low Dimensional 3D Descriptor.

3. S. M. Prakhya, B. Liu, W. Lin, V. Jakhetiya, S. C. Guntuku, "B-SHOT: A Binary 3D Feature Descriptor for Fast Keypoint Matching on 3D Point Clouds", Autonomous Robots, Available online 30 December 2016, DOI: 10.1007/s10514-016-9612-y .

- Contributes to Chapter 6: B-SHOT: A Binary 3D Descriptor.

4. S. M. Prakhya, W. Lin, V. Chandrasekhar, B. Liu, J. Lin, "Low Bit-rate 3D Feature Descriptors for Depth Data from Kinect-style Sensors", Signal Processing: Image Communication, Available online 27 November 2016, ISSN 0923-5965.

- Contributes to Chapter 7: Low Bit-rate 3D descriptors with Lattice Quantization.

\section{Conference Proceedings}

5. S. M. Prakhya, B.Liu, W. Lin and U. Qayyum, "Sparse Depth Odometry: 3D Keypoint based Pose Estimation from Dense Depth Data," IEEE International Conference on Robotics and Automation (ICRA), Seattle, WA, 2015, pp. 4216-4223.

- Contributes to Chapter 4: Sparse Depth Odometry. 
6. S. M. Prakhya, B. Liu and W. Lin, "B-SHOT: A Binary Feature Descriptor for Fast and Efficient Keypoint Matching on 3D Point Clouds," IEEE/RSJ International Conference on Intelligent Robots and Systems (IROS), Hamburg, 2015, pp. 1929-1934.

- Contributes to Chapter 6: B-SHOT: A Binary 3D Descriptor.

7. S. M. Prakhya, B. Liu, Y. Rui and W. Lin,"A Closed-form Estimate of 3D ICP Covariance," 14th IAPR International Conference on Machine Vision Applications (MVA), Tokyo, 2015, pp. 526-529.

\section{Technical Reports}

8. S. M. Prakhya, B.Liu and W. Lin, "On Creating Low Dimensional 3D Feature Descriptors with PCA," Research Gate (Available Online).

- Contributes to Chapter 5: 3DHoPD - A Fast Low Dimensional 3D Descriptor. 


\section{Chapter 1}

\section{Introduction}

\subsection{Background}

Traditional 2D cameras provide only a projective 2D representation of the real world that exists in three dimensions. During this projective transformation, depth information is lost, which causes a common problem in 2D computer vision domain known as scale ambiguity. Hence, to perceive the world in three dimensions as it is, 3D depth sensors are being developed. Moreover, 2D camera based computer vision applications such as image/object matching, recognition and segmentation assume that there is sufficient illumination and enough texture in the environment. Lack of illumination or texture render these systems impractical for any real world applications. Hence, 3D depth sensors that provide structure/depth information act as an alternative solution that resolves these challenges.

3D depth sensors estimate depth based on infrared (IR) or Time-of-flight (ToF) technologies. IR depth sensors have an IR projector that projects a dot-pattern, and an IR sensitive camera to capture this pattern. The depth is estimated from the displacement of this pattern based on the fact that the density of the pattern decreases as the distance increases. ToF sensors estimate the depth by calculating the time difference between the sent and the received signals. ToF sensors are relatively more expensive as they require high precision hardware to accurately predict the time difference between the sent and the received signals.

Examples of these depth sensors include Microsoft Kinect, Asus Xtion Pro Live, Google Tango [1], Structure sensor [2], Intel RealSense, laser scanners/ lidar sensors, Velodyne [3] and so on. Some of these sensors such as Microsoft Kinect and Structure sensors are aimed at indoor environments and provide dense depth data while others such as Velodyne are aimed for outdoors that work for long ranges, provide relatively sparse depth data and withstand severe weather conditions. Some of these depth sensors are shown in Fig. 1.1. 

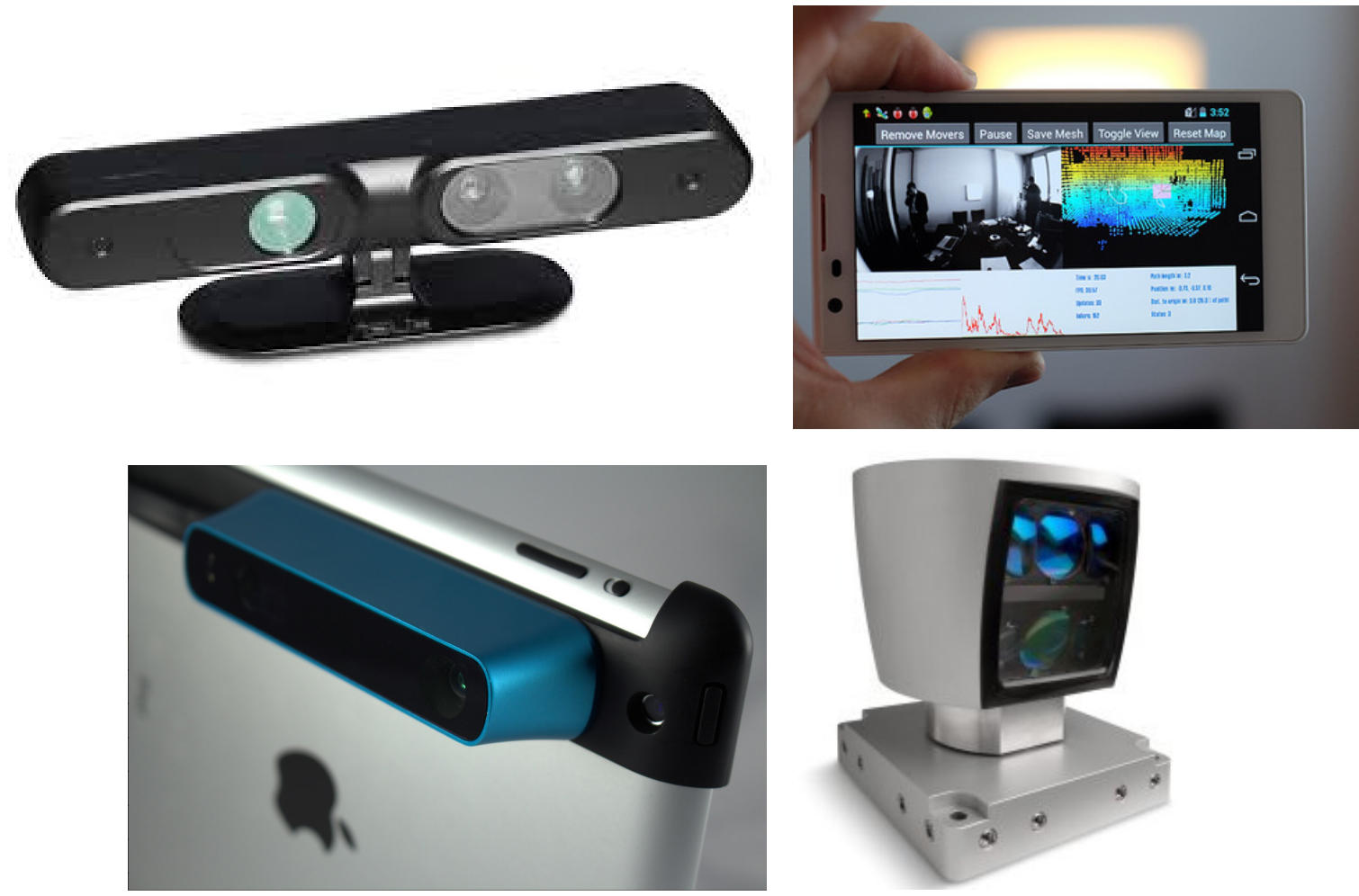

Figure 1.1: Various depth sensors are shown in this figure. Starting from top left, they are Asus Xtion Pro Live (top left), Google Tango (top right), Structure sensor (bottom left), Velodyne lidar (bottom right).

These depth sensors, such as Microsoft Kinect and Asus Xtion Pro Live are widely used in various computer vision [70] and robotic applications in indoor environments such as KinectFusion [103] and RGB-D SLAM [43], while lidar sensors such as Velodyne have played a keyrole in the success of self-driving cars [3].

$3 \mathrm{D}$ perception includes various applications such as 3D object recognition [63, 8], Simultaneous Localization and Mapping (SLAM)[44, 103, 43], Sparse Depth Odometry [111], 3D shape retrieval [129] and point cloud registration [65, 112]. In this thesis, we consider 3D perception applications that can run on mobile devices with these 3D depth sensors in indoor environments. Hence, it is extremely important to develop applications that are have both low computational requirements and low memory footprint.

In all these applications, the first step is $3 \mathrm{D}$ keypoint detection while the second step is $3 \mathrm{D}$ feature description.

3D Keypoint Detection: In this step, for example, an input point cloud from Microsoft Kinect consisting of three million points, is reduced to few hundreds or couple of thousands of keypoints. Hence, it is important that these 3D keypoints describe salient regions of the 
scene, as the accuracy of the next steps in the target application highly depends on this step. Therefore, efficient salient 3D keypoint detection is a prerequisite for developing accurate, computationally cheap and low memory demanding 3D perception applications.

3D Feature Description: Once the 3D keypoints are detected, the second step is to extract 3D feature descriptors. They essentially encode the neighbourhood surface patch consisting of few thousands of $3 D$ points into a multidimensional vector. 3D feature descriptors capture distinctive neighbourhood surface characteristics and hence can be matched using a distance metric such as Euclidean distance or Hamming distance to determine the patch similarity.

The following are the traits of a good 3D feature descriptor:

- It should encode the surface variantions/charactersistics in best possible way to make the keypoints distinguishable from the ones arising from non-matching surface patches but should be similar enough to match with the ones coming from same surface characteristics.

- It should be rotational invariant and to achieve this, a robust local reference frame is created and the neighbourhood patch is alighned to it.

- It should have low dimensionality (memory) and can be should be computed fast (computational).

- It should be robust to noise and existing works emply histogram based representations or signatures to represent spatial grids to achieve this.

More importantly, feature descriptor extraction and matching are some of the most computationally intensive and memory demanding steps in any target applications. While the memory footprint and the descriptor matching complexity scales accordingly with the descriptor's dimensionality, the descriptor extraction complexity depends on it's construction principles. Therefore, it of paramount importance to create extremely fast low dimensional real valued descriptors or even low bit-rate binary descriptors for mobile device applications.

After keypoint detection and feature description, in general, the extracted 3D feature descriptors are used to determine 3D keypoint correspondences based on a distance metric. Then the found matches are further processed to either remove false positives using RANSAC [53] or in the case of 3D object recognition, global hypothesis verification [7] is applied and so on. Various post processing steps are applied based on the target application.

In this thesis, we focus on the first two steps, i.e., 3D kepyoint detection and 3D feature description, and design salient 3D keypoint detectors and compact 3D descriptors that offer high performance while requiring lower memory and computational requirements. 


\subsection{Challenges in 3D Keypoint Detection}

As many 3D perception applications have keypoint detection and feature description as their first steps, these two modules greatly impact the accuracy, robustness, memory and computational footprints. Specifically, keypoint detection module reduces a few million points to a few hundred points, lessening the computational load on the subsequent modules as they only have to deal with those detected keypoints. However, it is extremely important to detect keypoints on meaningful and informative areas considering the target application.

\section{What is a keypoint cluster/set and how is it different from a distinct keypoint ?}

All the existing 3D keypoint detection methods detect distinctive keypoints. In these methods, a single keypoint is detected to represent a curved region. More importantly, every keypoint is separated from each other atleast by a distance of the radius used for non maxima suppression, i.e., every keypoint is distinctive and represents a curved region in the point cloud.

A keypoint set/cluster is a group of keypoints representing a highly curved region or a place, rather than a precise location. Instead of representing a curved region with a single keypoint whose position can vary drastically with noise, we represent the curved region with a group of keypoints. And these keypoint clusters/sets or groups of points collectively can be used to represent a point cloud in a comprehensive manner and provide a semantic structure.

It can happen that a specific application can find detection of keypoint clusters, which represent interesting regions as a whole, to be more meaningful, while another application may find distinctive keypoints, each one separated from the other by a certain distance to be more logical. Let us see some examples where keypoint clusters are more suitable over distinctive keypoints and vice versa.

\subsubsection{Keypoint Clusters for 3D Object Recognition}

For example, consider the application of 3D object recognition where we have a database of objects and the task is to match the incoming object model to the ones present in the database for recognition. In this scenario, graph matching between the input object model and all the database models can be a good solution, where the graph roughly describes the shape of the object. The task then boils down to creating a graph from the detected keypoints to efficiently describe the shape of the object. It is easy to create a shape graph if the object point cloud has keypoint clusters detected at highly curved regions on the object, rather than having distinctive keypoints that may arise from noise. Once we have cluster of keypoints at highly curved regions, then a graph can be easily created by considering the centroids of each cluster as nodes 
of the object shape graph. The advantage of a keypoint cluster representing a single node of the graph is that, even if a keypoint does not get detected, the centroid still remains close enough to enable graph based matching. However if a single keypoint misses out in the case of distinctive keypoints scenario, then the whole structure of the graph gets distorted. As 3D perception applications and specifically 3D object recognition in this case deals with noisy depth data, it of paramount importance to detect 'highly repetable' clusters of keypoints representing curved regions over distinctive keypoints that may sometimes vary with noise.

\subsubsection{Distinctive Keypoints for Pose Estimation}

In the previous example, we have seen that keypoint clusters can be a good option for 3D object recognition. However, let us consider an other general 3D perception application of pose estimation between two 3D point clouds. In this scenario, we have a source point cloud and a target point cloud and the task is to estimate the 3D transformation matrix between them, which is extremely relevant in applications such as $3 \mathrm{D}$ point cloud registration, alignment and odometry estimation. Here, the keypoints are either matched using 3D feature descriptors or just by nearest neighbour search for real time applications. In such kind of scenarios where keypoint matching happens with nearest neighbour search, distinctive keypoints offer accurate matching rather than keypoint clusters. The keypoint clusters detected on noisy point clouds provide slightly innacute matches when compared to disctinctive keypoints that are at a certain distance from each other, because of approximation to centroid and missing of few keypoints in the keypoint clusters. While keypoint clusters preserve the shape of the graph, distictive keypoints preserve the accuracy. Therefore, in the case of pose estimation, distinctive keypoints that lie at a certain distance from each other are more reliable and offer accurate matching.

\subsubsection{Unreliable Planar Keypoints}

Next, it is extremely important that the detected keypoints do not lie on planar regions because it is not possible to match planar keypoints from one point cloud to the planar keypoints on the other. This is because, no neighbourhood surface characteristics can be used to distinguish one from another which makes it impossible to find the exact match for a source keypoint from a set of target planar keypoints. Therefore it is extremely important that detected keypoints lie only on regions where there is some kind of neighbourhood surface variations that can be used to distinguish one keypoint from the another. 


\subsection{Limitations of 3D Feature Descriptors}

3D feature descriptors are multidimensional vectors that encode the neighbourhood surface charateristics around a detected keypoint. They essentially encode the information around the keypoint into a vector in a meaningful way that makes each keypoint highly distiguishable. Every keypoint consists of a single feature descriptor that is extracted around it and these feature descriptors are matched to find the keypoint correspondences. 3D feature descriptors impact the memory footprint of the target application as every keypoint has a single descriptor associated with it. Specifically, the dimensionality of the extracted descriptors dictate the memory footprint and the descriptor matching complexity while the hand crafted extraction method decides the extraction complexity.

Lack of Fast and Low Dimensional Real Valued 3D descriptors: A good number of 3D feature descriptors are already available in literature and Guo et. al [64] have performed a comparitive evaluation on them. The lowest dimensional 3D feature descriptor that is available in the literature is FPFH [124] which has 33 dimensions, where each dimension is a floating point value. Hence, it requires 33 x $4=132$ bytes or 1056 bits of memory. However, the biggest drawback of FPFH is that the extraction complexity shows an exponential rise with the increase in the density of the point cloud. Therefore, there is a need to develop 3D feature descriptors that are extremely fast and have much lower dimensionality to reduce the memory footprint.

Need to Create Low Bitrate 3D Descriptors: To further reduce the memory footprint and the computational requirements, binary descriptors are invented in 2D image domain. However, no binary $3 \mathrm{D}$ feature descriptors were created and main reasons could have been the fact that there is no equivalent attribute in $3 \mathrm{D}$ to intensity in $2 \mathrm{D}$, and the noisy $3 \mathrm{D}$ depth data. This highlights a good research direction of creating binary and lower bitrate 3D feature descriptors that can offer great reduction in computational and memory footprints.

\subsection{Thesis Structure: Motivation \& Contributions}

The thesis outline is shown in Fig. 1.2. The first part of the thesis consisting of Chapters 3 and 4 presents our work on 3D keypoint detection while the second part consisting of Chapters 5, 6 and 7 presents our work on 3D feature descriptors.

\section{D Keypoint Detection:}

- In Chapter 3, we propose a salient and highly repeatable 3D keypoint clusters detection algorithm that has well defined steps for salient region detection (planar keypoint removal) and pruning. 
- In Chapter 4, we consider a 3D perception problem, SLAM with an RGB-D camera and propose a new 3D keypoint detection module for it. Capitalizing on this new 3D keypoint detection module (for distinctive keypoints), we develop Sparse Depth Odometry (SDO) to estimate the ego-motion of an RGB-D camera solely from its depth data that can run in real time without a GPU.

\section{D Feature Description:}

- In Chapter 5, we propose a low dimensional, real valued, 3D feature descriptor, that is extremely fast to compute. At its core lies a novel 3D keypoint transformation technique based on local reference frame.

- In Chapter 6, we present the first binary 3D feature descriptor, B-SHOT, that can be used for generic 3D keypoint matching on 3D point clouds.

- In Chapter 7, we propose to employ lattice quantization and double stage lattice quantization to create even lower bitrate 3D descriptors than B-SHOT and yet offer significantly higher performance.

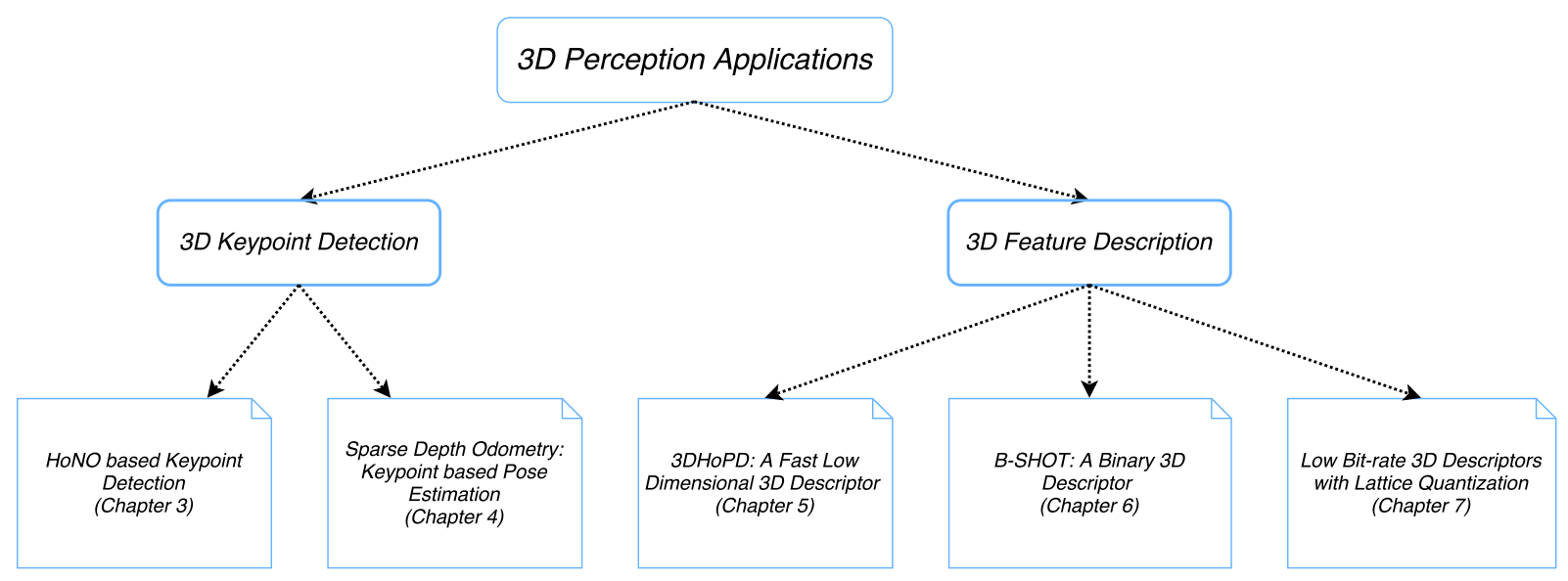

Figure 1.2: Thesis Outline

\subsubsection{Salient 3D Keypoint Detection}

In the first part of the thesis on 3D keypoint detection, we present a generic 3D keypoint detector that detects keypoint sets/clusters on 3D point clouds based on Histogram of Normal Orientations (HoNO) in Chapter 3. Next in Chapter 4, we consider a specific 3D perception 
application, Simultaneous Localization and Mapping (SLAM) with an RGB-D camera and propose a new 3D keypoint detection module that detects distinctive keypoints (aimed at nearest neighbour matching) and represents the scene comprehensively for pose estimation. Capitalizing on this new keypoint detection module, we present Sparse Depth Odometry (SDO) to estimate the ego-motion of a depth camera solely from its depth data and then create 3D maps of the environment.

\subsubsection{Histogram of Normal Orientations (HoNO) based Keypoint Detection}

In Chapter 3, we examined existing 3D keypoint detectors and found that they are either not robust enough to remove planar regions or do not detect highly repeatable keypoints. Hence, we propose a well defined two step 3D keypoint detection algorithm. In the first step, we remove all the non-salient regions from the input point cloud and pass only the salient or curved regions to the next step. In the second step, highly repeatable 3D keypoints are detected from those salient regions.

\section{Motivation: To detect highly repeatable keypoint clusters in salient regions.}

A 3D keypoint detector generally has two steps, firstly pruning and secondly non-maxima suppression (NMS). In the pruning step, most of the ineffective information is discarded while in the NMS step, yet another test is performed and detect the local maxima/minima as the final keypoints. Firstly, some of the existing 3D keypoint detectors do not have clearly defined pruning and NMS steps or integrate both into a single step, resulting in detecting less repeatable 3D keypoints. Secondly, these pruning and NMS steps are not robust enough and detect noise and glitches as keypoints. This happens because a less robust pruning step passes non-salient or planar regions to the NMS step. And as NMS is a locally defined criterion and checks for distinctiveness, a noisy point or glitch can thus get detected as a keypoint.

\section{Contributions: Robust Criterion for salient region detection and efficient pruning.}

In order to overcome the above mentioned drawbacks, we propose well-defined two step algorithm for keypoint detection.

- In the first step called as salient region detection, we propose to employ Histogram of Normal Orientations (HoNO), which is calculated at every point in the point cloud to avoid planar regions and successfully detect salient regions.

Next, as it is better to have more keypoints at high curvature or informative areas rather than having distinctive keypoints that may sometimes arise due to noise, we propose to find keypoint sets that are distinctive, depending on the target scene. 
- In the second step, which we call as pruning, the final keypoint sets/clusters are detected from the salient regions by evaluating the properties of both the HoNO and the neighbourhood covariance matrix.

\subsubsection{Sparse Depth Odometry: 3D Keypoint based Pose Estimation}

While in Chapter 3, we aimed at proposing a generic 3D keypoint sets detector, in Chapter 4, we look at a specific 3D perception application, SLAM with an RGB-D camera and propose a keypoint detection module for distictive keypoints. Capitalizing on the proposed 3D keypoint detection module, we developed sparse depth odometry to estimate the ego-motion of a depth camera solely from its depth data in real time without any use of GPU.

Motivation: To estimate the ego-motion of depth camera in real time without GPU.

With the availability of afforadable RGB-D cameras such as Microsoft Kinect, Asus Xtion Pro Live, there has been a proliferation of visual odometry algorithms [37, 137, 44, 155, 71] to estimate the ego-motion and create a dense 3D map of the environment. But these visual odometry methods fail to work if there is not sufficient and invariant illumination in the working environment. This is possible in long-term localization applications, disaster hit areas, human abandoned/dangerous places, tracking and mapping during night time, etc. As an alternative to visual odometry methods, there are methods that work with depth information alone, such as KinectFusion [103] and others [21, 17]. However, all of the depth alone methods follow the same trend of employing a signed distance function to represent the scene, which is computationally expensive and has a high memory footprint.

Challenges in the existing visual and depth based RGB-D odometry algorithms:

- Visual odometry algorithms require illumination and enough texture for their operation.

- Existing depth alone odometry methods such as KinectFusion and others employ Truncated Signed Distance Function (TSDF) to represent the scene, making the systems highly memory and computationally intensive, rendering them impractical to work without a GPU in real time.

Contributions: Sparse Depth Odometry which runs online without a GPU. At its core lies a novel 3D keypoint detection module that represents the scene comprehensively.

To overcome these drawbacks, we present Sparse Depth Odometry (SDO) that works with depth information alone, does not employ a signed distance function and runs online on a normal laptop with no GPU usage. SDO is inspired from visual odometry methods such as RGB-D 
SLAM [43] and CCNY RGB-D [37] that estimate ego-motion from 2D image keypoints such as SIFT and SURF detected in the scene.

- At its core, SDO is powered by a new keypoint detection module that comprises of two exisitng keypoint detectors SURE [51] and NARF [134].

We design neighbourhood structure test and neighbourhood variance test to evaluate how well different keypoint detectors represent a scene.

- Through this extensive evaluation and with theoretical justfication from the construction principles of SURE and NARF, it was shown that the proposed keypoint detection module comprising of SURE and NARF offers reliable keypoints that describe the scene more comprehensively, compared to others.

\subsubsection{Compact 3D Feature Description}

3D feature descriptors are the first resort to find keypoint correspondences between two arbitrarily oriented 3D point clouds. Moreover, 3D feature description is one of the most memory demanding and computationally expensive steps in most of the 3D perception applications. With the advent of mobile devices equipped with depth sensors, such as Google Tango and Lenovo Phab2 Pro, it is the need of the hour to develop fast (low computational) and less memory intensive $3 \mathrm{D}$ feature descriptors to power mobile applications.

Therefore, in this second part of the thesis, comprising of chapters 5, 6 and 7, we focus of developing compact 3D feature descriptors with low memory and computational requirements. Firstly, the Chapter 5 presents 3DHoPD, a fast real valued low dimensional descriptor, while the next one, Chapter 6 presents the first binary 3D descriptor B-SHOT. Lastly, Chapter 7 presents even lower bitrate and high performing compressed 3D descriptors than B-SHOT by applying lattice quantization on state-of-the-art real valued $3 \mathrm{D}$ descriptors.

\subsubsection{3DHoPD: A Fast low dimensional real valued 3D descriptor}

In the literature, there are either high dimensional 3D feature descriptors $[128,146,143,57,66]$ which require more memory or low dimensional descriptors [124] which require significantly higher computational time on dense $3 \mathrm{D}$ point clouds. Hence to cater for online and mobile device applications that have lower memory and power constraints, we develop a 18-dimensional 3DHoPD descriptor, that is extremely fast to compute.

Motivation: To develop a fast (less computations) and low dimensional 3D descriptor. 
The dimensionality of the 3D descriptor dictates the memory footprint and the descriptor matching complexity, while the descriptor extraction complexity depends on the hand crafted design of the descriptor.

- FPFH [124], being a 33 dimensional descriptor, has significantly higher extraction time when compared to other high dimensional descriptors on dense 3D point clouds because of its construction principles.

- On the other hand, SHOT (352 dim) and USC (1980 dim) are relatively faster in descriptor extraction compared to FPFH but demand higher memory footprint and matching complexity because of their dimensionality.

- More importantly, surface normal computation takes up most part of the computational power in the case of SHOT descriptor with 352 dimensions or other descriptors that employ it.

\section{Contributions: Proposal of, a new 3D keypoint transformation technique, a HoPD} descriptor and a tailored descriptor matching to remove upto $90 \%$ false positive matches.

To tackle the above challenges, we propose a low dimensional (18-dim) 3DHoPD that is extremely fast to compute and offers stable and competitive performance.

The novelty lies in the design of 3DHoPD (3D+HoPD):

- 3D: A new 3D keypoint transformation technique based on local reference frame is proposed to transform 3D keypoints to a new 3D space, where keypoints arising from similar 3D surfaces lie close to each other.

Hence capitalizing on the properties of this new 3D space, we propose a tailored descriptor matching technique in which, for a given source keypoint, a list of probable keypoint matches with high recall can be retrieved by a simple radial search. This reduces the search space by $90 \%$, removes false positive matches and aids in descriptor matching.

- HoPD: A fast HoPD (Histogram of Point Distributions) descriptor that does not involve surface normal computation is then proposed to find the exact match from this set of highly probable keypoint matches.

With this combination of a novel 3D keypoint transformation technique, HoPD descriptor, we propose 3DHoPD, a fast and low dimensional (18-dim) real valued descriptor. 


\subsubsection{B-SHOT: A Binary 3D Descriptor}

There are ample number of real valued 3D feature descriptors but there is no binary 3D descriptor for keypoint matching on 3D point clouds. Binary 3D descriptors can be matched extremely fast with Hamming distance while requiring extremely low memory. Hence, in Chapter 6, we present the first attempt in the literature to create a binary 3D descriptor.

\section{Motivation: To develop a binary 3D descriptor for generic 3D keypoint matching.}

- This high memory footprint in the case of real valued 3D descriptors arises from the fact that a single floating point value requires 4 bytes or 32 bits of memory according to IEEE floating point standards.

And that would require 1408 bytes of memory to store a single 352 dimensional SHOT descriptor. In applications that require storage of large number of descriptors, there would be a great surge in memory allocation.

- Next, matching the real valued descriptors with Euclidean distance also requires greater descriptor matching time, which is proportional to their dimensionality.

\section{Contribution: Adaptive binarization technique to create the first binary 3D descriptor.}

In order to cope with the above challenges, we propose an adaptive binarization technique to create the first binary 3D descriptor, B-SHOT, from a state-of-the-art real valued 3D feature descriptor, SHOT. The advantages of B-SHOT over the traditional SHOT feature descriptor are as follows.

- Firstly, SHOT, which is of 352 dimensions (requiring 1408 bytes) is reduced to 352 bits of binary data, therefore reducing the memory footprint, by 32 times.

- Secondly, keypoint matching can be performed much faster with Hamming distance metric when compared to real valued 3D feature descriptors (Experiments show a 6x improvement).

\subsubsection{Low Bit-rate 3D Descriptors with Lattice Quantization}

As mentioned before B-SHOT requires much lesser memory and computational power, however, B-SHOT offers relatively lesser keypoint matching performance when compared to SHOT. In this chapter, we propose to employ lattice quantization to create compressed 3D descriptors that require even less number of bits than B-SHOT and offer higher performance. 
Motivation: To compress even more than B-SHOT and yet improve the performance. In applications that require an input point cloud to be matched with a set of database point clouds present on a remote server, it is preferable to compress and transfer 3D feature descriptors online, rather than compressing and transferring the whole input point cloud. This is because the former would require much lesser bandwidth and does not require feature extraction on the server.

- Existing real valued 3D feature descriptors that offer good keypoint matching performance require higher bandwidth for their transfer over the network.

- On the other hand, the existing binary 3D feature descriptor, B-SHOT, requires relatively less bandwidth but offers reduced keypoint matching performance.

Hence, we propose to apply lattice quantization to compress state-of-the-art 3D descriptors.

Contributions: Apply lattice quantization to compress three state-of-the-art 3D descriptors and propose double stage lattice quantization to compress SHOT even more.

We propose to employ lattice quantization to compress three state-of-the-art real valued 3D feature descriptors, namely, SHOT [128], RoPS [66] and FPFH [124] and present their achievable keypoint matching performance. We perform compressed domain matching, where the compressed feature descriptors are matched by querying a pre-computed look up table, which is significantly faster than the widely used kd-tree [126] based matching technique. We also propose a double stage lattice quantization to compress the SHOT feature descriptor even further.

\subsection{Summary}

Keypoint detection and feature description are the two most important steps in most of the 3D perception applications. Moreover, these two steps greatly impact the accuracy, memory and computational requirements of the target application. With the advent of affordable depth sensors and mobile devices equipped with them, it is of paramount importance to have salient 3D keypoint detectors and compact 3D feature descriptors. Hence, in the first part of the thesis consisting of Chapters 3 and 4, we have focussed on developing salient 3D keypoint detection algorithms, while in the second part consisting of Chapters 5, 6 and 7, we have developed memory and computationally efficient 3D feature descriptors.

Salient 3D keypoint detection: Firstly in Chapter 3, we presented a generic 3D keypoint detector that detects salient and highly repeatable keypoint sets on $3 \mathrm{D}$ point clouds based on 
Histogram of Normal Orientations (HoNO). Next in Chapter 4, we considered a specific 3D perception application, SLAM with an RGB-D camera and proposed a new 3D keypoint detection module that represents the scene comprehensively for pose estimation. Capitalizing on this new keypoint detection module we presented Sparse Depth Odometry (SDO) to estimate the ego-motion of a depth camera solely from the depth data, without any need for RGB information, which can run in real time without GPU.

Compact 3D Feature Description: In Chapter 5, we presented 3DHoPD, a fast, real valued, low dimensional 3D descriptor. Next in Chapter 6, we proposed an adaptive quantization technique to create the first binary 3D descriptor B-SHOT. Lastly, in Chapter 7, we employed lattice quantization and proposed double stage lattice quantization to create even lower bitrate and high performing 3D descriptors than B-SHOT and presented a spectrum of possible bitrates and achievable performances.

Then, we concluded the thesis highlighting possible future directions of research. 


\section{Chapter 2}

\section{Literature Review}

\subsection{Modules in 3D Perception Applications}

In general a 3D perception application consists of various modules as shown in Fig. 2.1, such as 3D keypoint detection, 3D feature description, RANSAC, iterative closest point (ICP) algorithm and other post-processing steps.

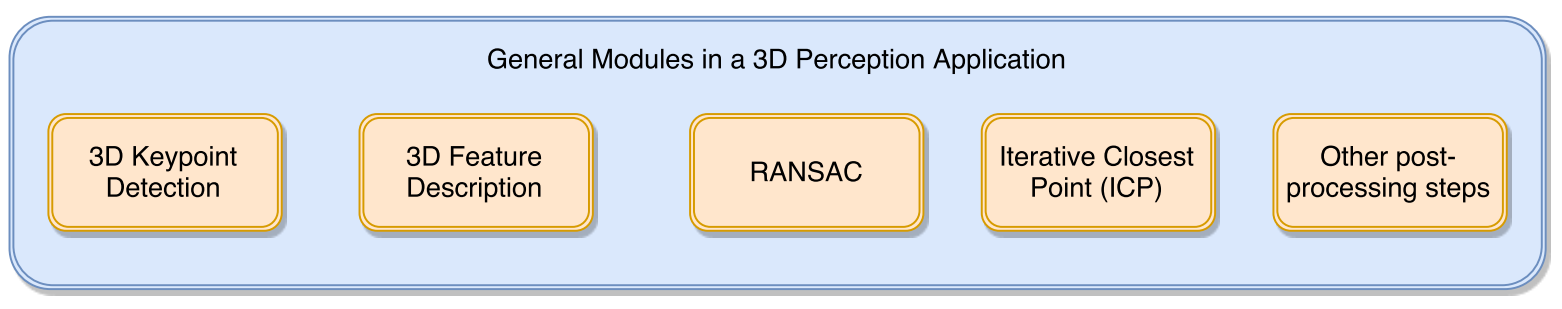

Figure 2.1: Various modules in 3D perception applications.

3D Keypoint Detection: Multiple points of interest are detected to represent salient and meaningful regions in the input point cloud. 3D keypoint detection reduces computational requirements of the following modules in a $3 \mathrm{D}$ perception application and determines its accuracy, as this step reduces a point cloud with millions of points to hundreds of keypoints.

3D Feature Description: In 3D feature description, multi-dimensional vectors (feature descriptors) are calculated to represent the neighbourhood surface characteristics around the detected keypoints. As these descriptors have to be computed for every detected keypoint, this step influences the computational requirements of a 3D perception application. Next, as these descriptors have to be stored for matching keypoints between a source and a target point cloud, they greatly influence the memory footprint of the 3D perception application as well.

The first part of this thesis presents salient 3D keypoint detection techniques while the second part presents compact and fast 3D feature description algorithms. 
RANSAC: RANdom SAmple Consensus (RANSAC) [53] is an iterative algorithm to robustly fit a mathematical model to a set of data containing outliers. It is also seen as an outlier removal method. The conventional RANSAC algorithm has two assumptions. First, there are few missing data, i.e., the inlier data points are good enough to estimate the underlying mathematical model. Second, there are few outliers, i.e., the outliers do not consistently agree with one mathematical model. The steps include, selection of a random set of data points based on which a model is estimated. The remaining data points are tested to check their consistency with the estimated model. If they agree with the estimated model within a tolerance level, then they form the consensus set. The estimated model is considered as a good model, if the consensus set has sufficiently large number of data points. Then a refined model is estimated using all the data points in the consensus set. These steps are interated multiple times and the mathematical model estimated from the largest consensus set is considered as the robust fit to the given set of data points. RANSAC acts an outlier removal method where the consensus set forms the inlier set of data points without any outliers. Various improvements $[114,30]$ have been proposed to cope with the assumptions and challenges of the traditional RANSAC.

In this thesis, we employ RANSAC algorithm to remove false feature descriptor correspondences and estimate the 3D transformation matrix from the inliers between two point clouds. 3D feature descriptor based keypoint matching generates some false correspondences, and RANSAC is heavily employed in computer vision domain to remove those false correspondences and return an inlier set to robustly estimate the mathematical model.

Iterative Closest Point (ICP): ICP [13] algorithm is employed to finely register a source point cloud to a target point cloud. Traditional ICP [13] algorithm assumes that both the point clouds are closely aligned. In many practical applications, the point clouds are first aligned based on the 3D transformation estimated from RANSAC, and then ICP algorithm is employed to accurately register both the point clouds. ICP finds a nearest point in the target point cloud for every point in the source point cloud. Then based on these correspondences, the 3D transformation (both rotation and translation) between the point clouds is estimated based on an error metric. The source point cloud is then transformed based on the found estimate and the process is iterated until the alignment error is less than a threshold or for a fixed number of iterations. Various error metrics [123] have been proposed to suit different target applications [108]. Recently with the proposal of Go-ICP [156], the prerequisite for traditional ICP that the point clouds need to be closely aligned has been solved.

Other post-processing steps: These mainly include various voting schemes [62, 152, 14] and hypothesis verification [7] techniques that vary depending on the target $3 \mathrm{D}$ perception applications. 


\subsection{Related Work}

As described in the previous chapter, the first part of this thesis is on 3D keypoint detection, while the second is on $3 \mathrm{D}$ feature description.

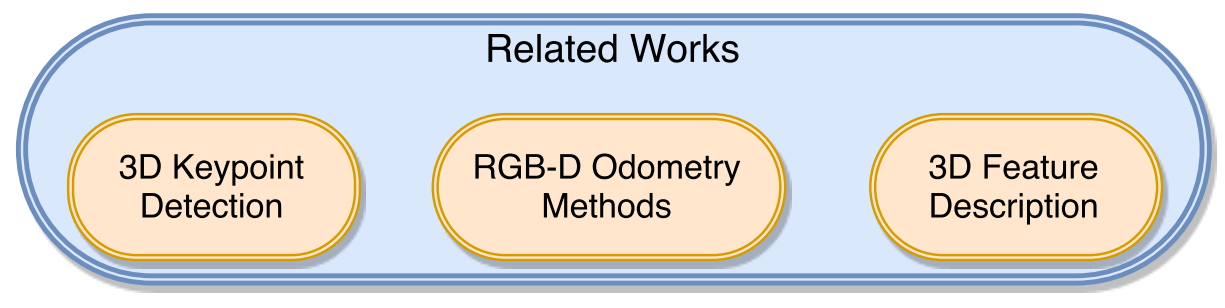

Figure 2.2: Outline of related works presented in this section.

In this thesis, we firstly propose a general purpose salient 3D keypoint detection algorithm that finds repeatable keypoint sets.

- Hence, we first present the existing works on 3D keypoint detection, as shown in Fig. 2.2.

Next, in the thesis, we consider a specific 3D perception application SLAM with an RGB-D camera and propose a solution, Sparse Depth Odometry (SDO), where the main contribution lies in the proposal of new keypoint detection module for this application.

- Therefore, as shown in Fig. 2.2, we present related works on RGB-D camera based odometry in the second part of this chapter.

Finally, this thesis focusses on presenting our attempts towards creating compact 3D descriptors, with the proposal of, a fast low dimensional real valued 3D descriptor, the first binary 3D descriptor and even low bitrate 3D descriptors that offer better performance.

- Therefore, as shown in Fig. 2.2, we discuss existing 3D feature descriptors in the last part of this chapter.

\subsection{D Keypoint Detectors}

A keypoint detector reduces an input point cloud to fewer number of keypoints, which helps in improving the accuracy and reducing the computational requirements of the target application. While there are a few works on comparitive evaluation of 3D keypoint detectors $[49,48,145,48]$, the work by Tombari et. al [145] offers a more comprehensive performance evaluation compared to others. Existing 3D keypoint detectors can be classified either as fixed 
scale $[25,162,96,140]$ or adaptive scale $[150,160,22,72]$ keypoint detectors. A fixed scale keypoint detector works on a constant scale whereas an adaptive scale keypoint detector generates multiple scales of the input point cloud and detects keypoints on various scales.

Adaptive scale 3D keypoint detectors are motivated by the success of the SIFT keypoint detector ([90]) in the 2D image domain. It is to be noted that generating a scale space representation of a 3D point cloud deforms the 'structure' of the input point cloud. A fixed scale keypoint detector operates on a local neighbourhood of radius $\epsilon$ at a constant scale. It can also be seen that, varying the parameter $\epsilon$ would enable a fixed keypoint detector to detect keypoints at various scales. Our proposed keypoint detection algorithm is a fixed scale one and hence we review the existing fixed scale 3D keypoint detectors.

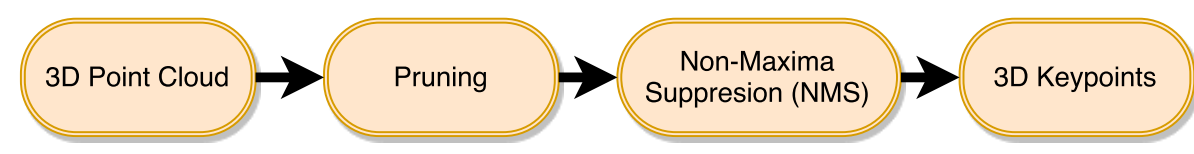

Figure 2.3: Pipeline of a fixed-scale 3D keypoint detector.

Fixed scale 3D keypoint detectors: As mentioned by [145], fixed-scale keypoint detectors $[25,162,96,140,51,134]$ generally comprise of two steps. First one is a pruning step and the second one is a non-maxima suppression (NMS) step, as shown in Fig. 2.3. In the pruning step, most of the ineffective information is discarded based on some criteria and the informative points are passed to the next stage. In the NMS step, yet another test is performed in a radius of $R_{n m s}$ over the informative points to detect the final keypoints that are distinctive and are at least a distance of $R_{n m s}$ away from each other.

Local Surface Patches (LSP) [25] employed Shape Index metric (SI)([35]) to prune noninformative points and find the salient points. In the NMS step, only those salient points, which attain either a local maxima or minima in terms of SI are labelled as keypoints. Intrinsic Shape Signatures (ISS) $[162,145]$ keypoint detector uses the two successive eigenvalues of the neighbourhood covariance matrix for pruning while considering the smallest eigenvalue for NMS. KeyPoint Quality (KPQ) [96] detector employed a relaxed pruning step to detect more salient points when compared to ISS keypoint detector, and used curvatures in the NMS step to detect final keypoints. SURE [51] keypoint detector works by calculating the entropy of surface normal histograms and their variance in a local neighbourhood to detect keypoints.

NARF [134] keypoint detector generates a range image and finds keypoints at stable and curved regions by moving the keypoints away from the object boundaries present in the scene. SDTP keypoint detector [153] calculates interest values by employing signed distances on a 
tangent plane and a surface curvature based NMS to detect keypoints. While the research is heading towards developing more robust and repeatable keypoints, interestingly, [6, 5] have showed that by employing game-theoretic matching of simple surface 3D keypoints, a robust surface registration can be achieved even without any initial estimate, which is pre-requisite for ICP $[123,148]$ algorithm.

\subsection{RGB-D Camera based Odometry}

In this section, we present a review of existing RGB-D camera based odometry methods.

Almost all the notable works on RGB-D camera based odometry can be classified into two categories, as shown in Fig 2.4. Firstly $R G B+$ Depth, methods that use both RGB and depth data. Secondly Depth alone, ones that use depth data alone for pose estimation. Furthermore, all the works in $R G B+$ Depth can be divided into two sub-groups, namely sparse methods and dense methods. Sparse methods [44, 71, 37, 76], as will be discussed in Section 2.4.1, extract keypoints on RGB images and track them across frames. Dense methods [79, 155] that use all of the available information, as will be discussed in Section 2.4.2, minimize the photometric error on RGB and depth images between consecutive frames for pose estimation. In simple words, sparse methods reduce the RGB and depth images to a few keypoints, while dense methods use all of the available RGB and depth information for pose estimation. Coming to the Depth alone category, all the existing works use all of the available depth information by employing SDF (signed distance function), thus falling under the Dense Methods category.

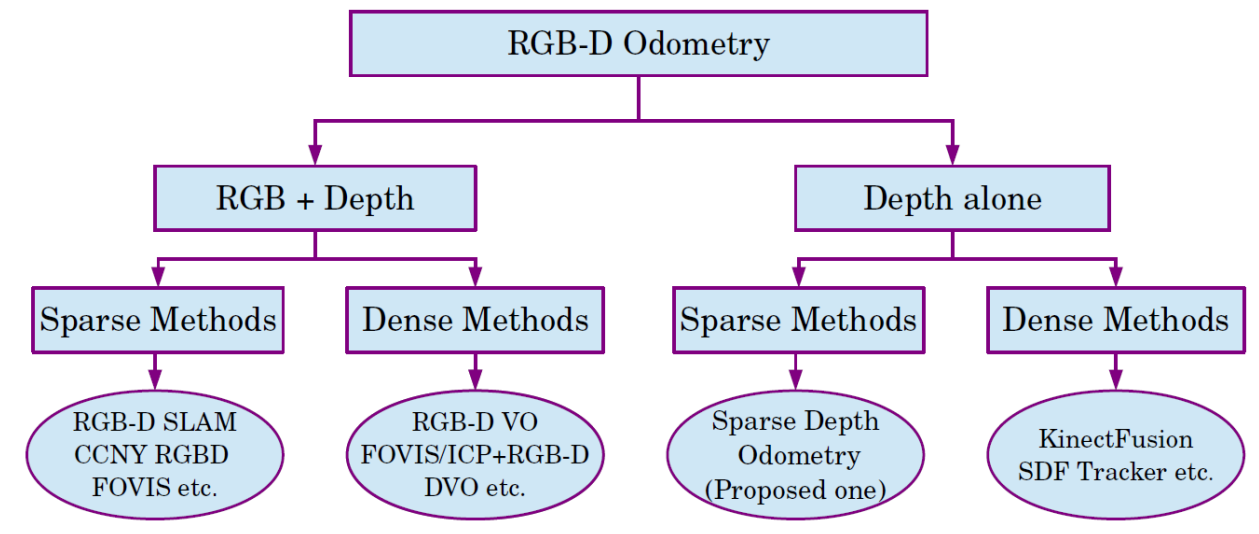

Figure 2.4: General Classification of RGB-D Camera Based Odometry Methods

\subsubsection{Sparse methods that use RGB and depth information}

The first step of all the works in Sparse Methods under RGB+Depth as shown in Fig. 2.4 is to extract keypoints such as SIFT [90], SURF [11], FAST [120], ORB [122] on RGB images. 
Endres et al. [44, 43] used feature descriptors to match keypoints, removed false correspondences using random sample consensus (RANSAC) and estimated the pose of the sensor. Loop closures were detected and the pose graph was optimized to build a 3D map. Henry et al. [71] developed a similar algorithm, except that they used transformation estimated from the matched keypoints to initialize the ICP (iterative closest point) algorithm and employed sparse bundle adjustment instead of graph optimization. Huang et al. [76] also used keypoint detection, then computed a graph of consistent feature matches and used a greedy algorithm to approximate the maximal clique in the graph to detect inliers. Dominguez et al. [34] designed a filter that keeps track of stable and reliable features after feature matching. Dryanovski et al. [37] extracted keypoints on RGB-D images and used ICP to register them with a model of keypoints. Their main contribution lies in the design of a Gaussian kernel to represent the noise in depth images more accurately at the edges and corners present in the scene.

\subsubsection{Dense methods that use RGB and depth information}

Here, we discuss the algorithms that fall in Dense Methods under RGB+Depth as shown in Fig. 2.4. Stuckler et al. [137] integrated depth and color cues into a multi-resolution surfel map representation (MRS). They built MRS maps from RGB-D data, considering the measurement noise characteristics, and employed an ICP variant to register these maps. Steinbruecker et al.[135] formulated the maximization of photo-consistency between two frames as a nonconvex problem. They linearized the non-convex problem and solved it by sequential convex optimization. Meanwhile, Kerl et al. [79] minimized the photometric error between consecutive RGB-D frames and estimated pose accordingly. Whelan et al. [155] implemented Steinbruecker's method [135] on a GPU and fused it with FOVIS [76] and Kintinuous [154]. The aim behind fusing these works was to handle the problem of Kintinuous [154] failing in environments with less structure information.

\subsubsection{Dense methods that use depth information alone}

The existing works in Dense Methods under Depth alone as shown in Fig. 2.4, use the truncated signed distance function (TSDF) to represent the underlying structure of the scene. The first work by Newcombe et al. [103] called KinectFusion, represented the scene using a TSDF model. A point cloud was generated from the TSDF model and was registered with the incoming point cloud in a course-to-fine manner using ICP. One of the limitations of KinectFusion is that it can only operate in small workspaces. This issue was solved in [154] and [121] by 
dynamically modifying the TSDF model, hence enabling it to work in large scale. Unlike the above approaches where TSDF was used only to represent the scene, [21] and [17] estimated the pose directly by minimizing the error on depth images using TSDF.

Our proposed method SDO works by extracting keypoints on dense depth data from Kinectlike sensors, thus falling under Sparse Methods in the Depth alone category of Fig. 2.4. SDO is inspired by existing sparse $\mathrm{RGB}+\mathrm{D}$ methods and our main contribution lies in the design of an efficient keypoint detection module that when combined with SDO, offers performance comparable to the state-of-the-art.

\subsection{D Feature Description}

In this section, we review the existing 3D feature descriptors.

Guo et al. [64] have performed a comprehensive evaluation of existing 3D feature descriptors and highlighted three state-of-the-art 3D feature descriptors, namely SHOT [128], RoPS [66] and FPFH [124] with the following findings:

- For time-crucial and memory constrained applications, FPFH [124] is the best option on low density point clouds, as it is only of 33 dimensions and offers fair feature descriptor matching. However, on high density point clouds, the computational requirements of FPFH show an exponential rise [128], which is not desirable.

- SHOT [128], a 352 dimensional feature descriptor, is the best option on high density point clouds, as it offers good trade-off between keypoint matching accuracy and computational efficiency.

- RoPS [66], a 135 dimensional descriptor, is the best option if the characteristics of the input point cloud are unknown, as it has offered stable performance in all test settings.

We extensively use, modify and compare our proposed compact 3D descriptors with SHOT, RoPS and FPFH descriptors. Hence, next, we briefly present the construction principles of these three state-of-the-art descriptors.

\subsubsection{FPFH: Fast Point Feature Histograms}

FPFH [124] was proposed as an improvement to PFH [125] feature descriptor, wherein the computational complexity in computing a single descriptor was reduced from $O\left(n^{2}\right)$ to $O(n)$, 
with $n$ representing the number of points in the support region. In FPFH, firstly a simple point feature histogram (SPFH) is computed for all the keypoints, based on the Darboux frame estimated between the considered keypoint and its $n$ neighbourhood points from the support region. Then, the final FPFH descriptor at a keypoint $q$ is calculated based on weighted summation of the SPFH values of the $n$ neighbourhood points, i.e.,

$$
F P F H(q)=S P F H(q)+\frac{1}{n} \sum_{k=1}^{n} \frac{1}{\alpha_{k}} \cdot \operatorname{SPFH}\left(q_{k}\right)
$$

where, $\alpha_{k}$ is the distance between the considered keypoint $q$ and its neighbourhood points $q_{k}$ with $k=\{1,2, \ldots, n\}$. While PFH [125] used joint histogram representation, FPFH employed separate histograms for each feature dimension to decorrelate them, resulting in a 33 dimensional vector. Though FPFH requires less computations as compared to PFH, it effectively uses neighbourhood points that lie outside the support region, upto $2 \mathrm{x}$ of the defined support radius. Hence, with an increase in the support radius or the density of the point cloud, FPFH shows a drastic rise in its computational demands.

\subsubsection{SHOT: Signatures of Histograms of OrienTations}

SHOT [128] is a hybrid feature descriptor that leverages on the advantages of both signature and histogram based methods [128]. It creates a 3D spatial grid with 32 partitions arising from 8 azimuth, 2 elevation and 2 radial divisions, as shown in Fig. 2.5 (note that only 4 azimuth partitions are shown for better visibility) around a keypoint and then represents the variations of surface normals present in each spatial grid by a histogram.

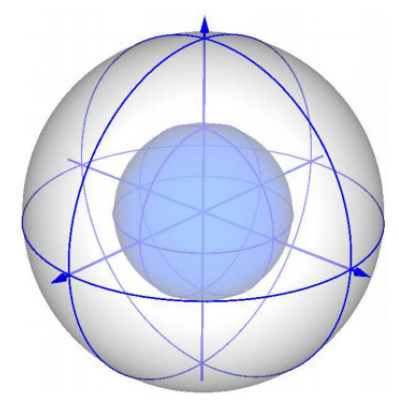

Figure 2.5: Representative spherical Grid employed in SHOT [128] descriptor.

Firstly, affine invariance is achieved by creating a local reference frame from the eigenvectors of the modified neighbourhood covariance matrix $C$. The sample points $q_{i}$ that lie in the 
support region of radius $\mathbf{r}$ are weighed based on their distance from the considered point $\mathbf{q}$, as shown in Eqn. 2.2.

$$
\mathbf{C}=\frac{1}{\sum_{i: d_{i} \leq \mathbf{r}}\left(\mathbf{r}-d_{i}\right)} \sum_{i: d_{i} \leq \mathbf{r}}\left(\mathbf{r}-d_{i}\right)\left(\mathbf{q}_{\mathbf{i}}-\mathbf{q}\right)\left(\mathbf{q}_{\mathbf{i}}-\mathbf{q}\right)^{\mathbf{T}}
$$

where $d_{i}=\left\|q_{i}-q\right\|_{2}$.

Secondly, the histograms representing each of the 32 spatial grids, are created by binning the cosine of the angle between the estimated local reference frame and the surface normals of the points that lie in the considered spatial grid. Moreover, uniform binning of the cosine angle is equivalent to applying a coarse to fine binning over the range, hence making the feature descriptor robust to small variations in surface normals due to noise. To cope with the boundary effects arising from histogram based binning and small perturbations in local reference frame, quadrilinear interpolation technique is employed. Finally the descriptor is normalized to make it robust against point cloud density variations.

\subsubsection{RoPS: Rotational Projection Statistics}

RoPS [66] capitalizes on creating a robust local reference frame based on the eigenvalue decomposition of the neighbourhood covariance matrix, by incorporating the additional mesh connectivity information. And the rotational invariance is achieved by aligning the local surface patch to the estimated local reference frame. Then the local surface patch is rotated around the $x, y, z$ axes while projecting the points onto $y z, z x$ and $x y$ planes. From each projection, a distribution matrix that captures the point distributions is constructed and its statistics, such as central moments for descriptiveness and Shannon entropy are calculated. Subsequently, these statistics arising from all the rotations around the $x, y, z$ axes and projections onto $y z, z x$ and $x y$ planes are concatenated to create a 135 dimensional RoPS descriptor.

\subsubsection{Other Real Valued 3D Feature Descriptors}

Point Signatures [29] inserts a 3D sphere at a keypoint $p$ and estimates the curve $C$ that crosses the $3 \mathrm{D}$ surface. The descriptor is created from the signed distance profile that arises by moving the tangential plane at the point $p$ in the direction of its normal. In [104], the authors use exponential mapping to map the neighbourhood points to a $2 \mathrm{D}$ plane and then create a feature descriptor by encoding the surface normals in the support region. In LD-SIFT [33], a SIFT descriptor [90] is computed on a 2D array representing the distance of each point in the support region to the locally fitted plane. SURF descriptor [11] from 2D image domain is also extended 
to $3 \mathrm{D}$ by calculating Haar wavelet responses on voxelized 3D meshes and is dubbed as 3D SURF [81]. Spin Image descriptors [78] are created by counting the number of points that lie in the radial and vertical grids of the cylindrical volume around the considered point. This cylindrical volume is aligned with the point normal while weighing and interpolation schemes are employed for further enhancement. Local Surface Patch [25] feature descriptors capture the surface type, centroid of the considered patch and a histogram of shape indexes [36] vs. neighbourhood normal angles with respect to the center point normal, in its descriptor.

3D Shape Context [57] feature descriptor creates histograms from spatial grids in the 3D spherical volume around the considered point and weighs them based on the grid volume and local point density. Unique Shape Context [143], enhances 3D Shape Context by employing a local reference frame created from the eigenvalue decomposition of the neighbourhood covariance matrix and aligning the neighbourhood points accordingly to achieve rotational invariance. The Radius based Surface Descriptor (RSD) [93, 92] calculates the distance between the keypoint and its neighbourhood points and the angle between their normals. From these, the ones with the maximum and minimum radii are considered to create the descriptor.

THRIFT [55, 54] creates a histogram of angle differences between the surface normals at the keypoint and the neighbourhood points, and the weights of each neighbourhood point depends on the density and its distance from the keypoint. Tri-Spin-Image (TriSI) [67, 68] combines RoPS [66] and Spin Image [78] descriptors. Firstly, rotational invariance is achieved by transforming the local patch based on the estimated local reference frame and then three spin image descriptors are estimated by considering the $x, y$, and $z$ axes as the local reference axes. These three spin images are concatenated to form the TriSI descriptor. However, as it uses the same local reference frame estimation as in RoPS, it requires mesh connectivity information as well for its application. There is another interesting biologically inspired keypoint detection method, BIK-BUS [50] that employs a computationally intensive method of visual saliency detection. The saliency maps are estimated in a bottom-up fashion and is data driven to move towards most interesting/salient points in the map. Though it offered good performance in combination with various 3D feature descriptors, its computational cost makes its deployment in real time applications almost impossible. However, if accuracy is the highest priority compared to real time operation, then BIK-BUS [50] is one of the good options.

The chapters $6 \& 7$ present our works on binary and compressed 3D descriptors. The relevant $2 \mathrm{D}$ binary descriptors and compression techniques are reviewed at that point, in those chapters. 
With this, we conclude this chapter on reviewing relevant work on 3D keypoint detection, RGB-D camera based odometry methods and 3D feature descriptors. In the next section, we present our first work, Histogram of Normal Orientations (HoNO) based keypoint detection algorithm to detect salient and repeatable keypoint sets on 3D point clouds. 


\section{Chapter 3}

\section{Histogram of Normal Orientations (HoNO) based Keypoint Detector}

\subsection{Introduction}

In this chapter, we propose a fixed-scale keypoint detection algorithm to detect salient and repeatable keypoint sets on 3D point clouds. The relevant related work on 3D keypoint detection was reviewed in Sec. 2.3 of the previous chapter. A comprehensive performance evaluation of 3D keypoint detectors was performed by Tombari et. al [145], moreover, they also provide the experimental framework through which, new 3D keypoint proposals can be compared against the existing ones.

In general, a fixed-scale keypoint detector generally comprises of two steps. First one is a pruning step and the second one is a non-maxima suppression (NMS) step, as shown in Fig. 2.3. In the pruning step, most of the ineffective information is discarded based on some criteria and the informative points are passed to the next stage. In the NMS step, yet another test is performed in a radius of $R_{n m s}$ over the informative points to detect the final keypoints that are distinctive and are at least a distance of $R_{n m s}$ away from each other. The NMS step works locally and detects the local minima or maxima based on the method's criteria as the final keypoints. If non-salient or less informative regions are passed to the NMS step, then noise or glitches in 3D data may get detected as keypoints, because they tend to exhibit distinctive characteristics locally, which is not desirable.

Existing keypoint detectors as presented in Sec. 2.3, either do not detect salient regions or they do not employ robust criteria for pruning and NMS. Later in this chapter, in Fig. 3.3 and Fig. 3.4, it was shown that ISS and KPQ keypoints are not robust and the detected keypoints lie on planar regions. Therefore, we propose a new 3D keypoint detector that decouples salient region detection and pruning, and provide robust criteria for both. The proposed salient region 
detection step efficiently removes planar or non-informative regions from the input point cloud, while the pruning step condenses the salient regions to sets of keypoints. Moreover, it is better to have more keypoints at high curvature or informative areas rather than having distinctive keypoints that may sometimes arise due to noise, because NMS step works locally as mentioned before. Hence, contrary to the existing norm that each and every keypoint should be distinctive, we propose to find keypoint sets (groups of keypoints) that are distinctive, depending on the target scene. We show that the detected keypoint sets are more repeatable than the relevant existing ones on 3D keypoint detection benchmark datasets.

The detected keypoints essentially represent informative 'places' and 'points' present in the scene. This also opens a new research direction that aims at establishing accurate 'place' matches by developing feature descriptors that employ information from neighbouring keypoint sets. In the next section, we describe the proposed method for 3D keypoint sets detection.

\subsection{The Proposed Keypoint Detector}

There are two main steps in the proposed keypoint detector. Firstly, salient region detection that removes planar regions and retains only the curved/informative regions in the input point cloud. Secondly, a pruning step that reduces the salient regions to final keypoint sets. In these two steps, we employ a technique called HoNO (Histogram of Normal Orientations), which we will first discuss, and later explain the two steps in detail. Though similar techniques to HoNO were used for creating 3D descriptors ([128, 146, 144]), our novelty lies in employing them for 3D keypoint detection by estimating their peakedness from the kurtosis of the histogram.

\subsubsection{HoNO: Histogram of Normal Orientations}

At every point $p_{i} \in P$ (input point cloud), a normal $N_{i}$ is calculated by approximating it to the smallest eigenvector of the neighbourhood covariance matrix $C_{i}$ calculated from the points $p_{j}$, where $\left\|p_{j}-p_{i}\right\|<R_{\text {normal }}$, and $R_{\text {normal }}$ is the radius used for normal computation. After computing the normals $N_{i} \forall p_{i}$, a histogram $H_{i}$ that represents the orientations of neighbourhood normals $N_{j}$ with respect to $N_{i}$ is calculated for every point $p_{i}$, where $N_{j}$ are the normals that belong to the neighbourhood points $p_{j}$, such that $\left\|p_{j}-p_{i}\right\|<R_{H o N O}$, while $R_{H o N O}$ is the radius used for HoNO computation. The orientation of a specific normal $N_{q}$ with respect to $N_{i}$, where $N_{q} \in N_{j}$, represented by an angle $\theta_{\text {deg }}$ is calculated as shown in Equation 3.1.

$$
\theta_{\text {deg }}=\left(\arctan \frac{\left\|N_{i} \times N_{q}\right\|}{N_{i} \cdot N_{q}}\right) * \frac{180}{\pi}
$$


where $\times$ represents the cross product and $\cdot$ represents the dot product between two vectors.

The angle $\theta_{\text {deg }}$ lies between 0 to 180 as $N_{i} \cdot N_{q}$ is always non-negative. The angle $\theta_{\text {deg }}$ is used to fill a histogram $H_{i}$ of 18 bins with each bin size of 10 degrees. After binning the angles of all neighbourhood normals, the histogram is normalized to make it independent of neighbourhood density. Please note that each point $p_{i} \in P$ will have an histogram $H_{i}$ created from its neighbourhood normal orientations. Hence we call the histogram $H_{i}$ as HoNO (Histogram of Normal Orientations). Each bin of $H_{i}$ has a width of 10 degrees and gives a count of neighbourhood normals that fall into a specific bin.

In short, this HoNO $H_{i}$ at every point $p_{i}$ represents the spatial diversity of normals that are present in the neighbourhood of $p_{i}$. It can be easily seen that a point with a planar neighbourhood will have a histogram whose bin values will be high in the first bin and a zero value in rest of the bins. In the same way, a highly curved area will have most of the bins occupied in its histogram as it will have diversely oriented normals in its neighbourhood. To quantitatively measure the peakedness of HoNO, which represents the neighbourhood normals' spatial diversity, we employ the kurtosis of an histogram as shown in Equation 3.2 ${ }^{1}$.

$$
\begin{aligned}
\operatorname{Kurt}(H) & =\frac{\sum_{k=1}^{N}\left(H_{k}-\bar{H}\right)^{4}}{N S^{4}}-3 \\
S & =\sqrt{\frac{\sum_{k=1}^{N}\left(H_{k}-\bar{H}\right)^{2}}{N}}
\end{aligned}
$$

where $H$ is the histogram under consideration, $\mathrm{N}=18$ in our case as the considered histogram has 18 bins, $H_{k}$ represents the count in the $k^{\text {th }}$ bin of $H, \bar{H}$ represents the mean of all the bins in $H$ and $S$ represents the standard deviation of histogram bins, as shown in Equation 3.3.

\subsubsection{Keypoint Detection}

In this section, we describe the salient region detection step and the pruning step that employ HoNO and some neighbourhood properties, to detect keypoints on the input point cloud.

Lets represent the input point cloud as $P$. We first compute normals and calculate HoNO's for each point $p_{i} \in P$. As explained in Section 3.2.2.1, salient regions in the input point cloud $P$ are detected and those salient points that lie closer to boundaries are removed. Finally, as explained in Section 3.2.2.2, a pruning step is applied on the detected salient regions to find keypoint sets. These two steps, namely salient region detection and pruning are illustrated in Fig. 3.1 on a scene scene_005 from the Kinect dataset. The detected salient regions are visualized in Fig. 3.1(b) while the final keypoint sets after pruning are shown in Fig. 3.1(c).

\footnotetext{
${ }^{1}$ We use the 'excess kurtosis' formula of an histogram that reduces the kurtosis of a standard normal distribution to zero.
} 


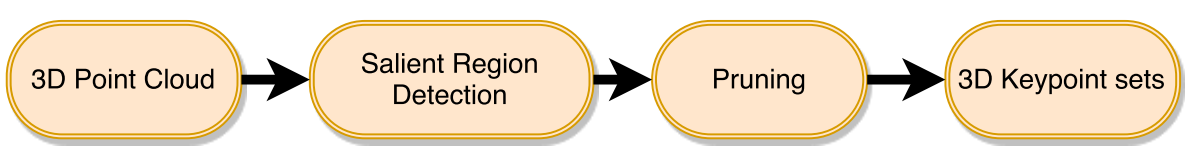

The proposed pipeline for keypoint sets detection.

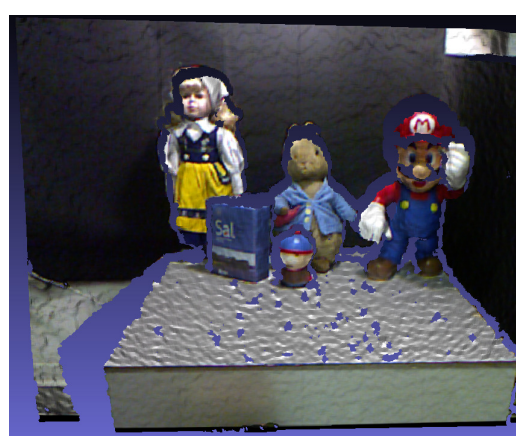

(a) Input Point Cloud

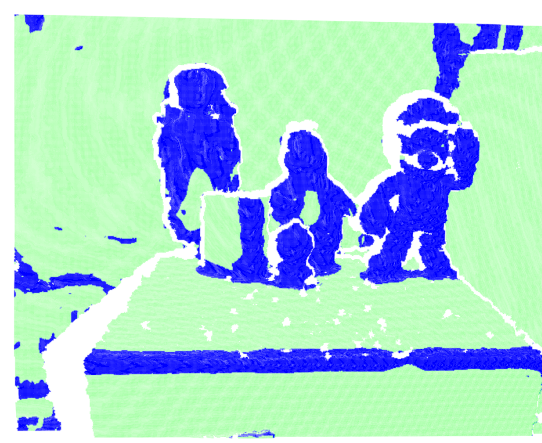

(b) Salient Region Detection(c) Final Keypoint Sets after Pruning

Figure 3.1: Keypoint sets detected by the proposed approach on scene_0 05 from the Kinect dataset, which is proposed by [145].

\subsubsection{Salient Region Detection}

We first compute normals $N_{i} \forall p_{i}$ and calculate a HoNO $H_{i}$ for each point $p_{i} \in P$ (input point cloud). The input point cloud is scanned to remove planar or less curved regions as it is unreliable to extract keypoints in those areas. This is performed by thresholding the kurtosis of HoNO at every point. The points in the point cloud whose histograms have a value of kurtosis higher than a threshold $T h_{K}$ are not passed to the next stages because they represent planar or flat regions. In our case, we are interested in the regions that are more curved in nature, hence we are looking for histograms that have a wider spread, or in other words, less-peaked histogram. A less-peaked histogram is constructed from a neighbourhood that has more diversely oriented surface normals, which represents a curved surface. The crafted technique of using the kurtosis of HoNO, fares well with the noisy data provided by Kinect-style depth cameras and it removes noisy planes and unreliable surfaces on the objects.

The salient regions that lie close to the boundaries are also removed because the data provided by various sensors is extremely noisy in boundary regions. The boundary extraction routine is implemented as mentioned in the PCL library ([126]). A parameter called boundary radius $R_{B}$ is used to determine if any point from the salient region lies close to a boundary. If yes, then that point is discarded from the salient region. 


\subsubsection{Pruning Step}

Let $d_{i}$ represent the set of points from salient regions, which are passed from the previous step. For every point $d_{i}$, we check for two conditions to label it as a keypoint or not. The first condition is that in the neighbourhood of radius $R_{d}$ around $d_{i}, d_{i}$ should have the lowest value of the kurtosis of HoNO. It means that at the current point $d_{i}$, the change in normals reaches a local maximum. Next, in the second condition, we check if $d_{i}$ has the strongest normal, i.e., if $d_{i}$ has the largest value for the smallest eigenvalue of the neighbourhood covariance matrix $C_{d i}$, compared to all the points that lie in radius $R_{d}$. If any of these two conditions hold, when compared to all the neighbourhood points, then $d_{i}$ is labelled as a keypoint.

One way of looking at our proposed methodology is that we employed a robust criterion for saliency detection and used a relaxed non-maxima suppression by adding an extra condition. To not confuse the readers with the taxonomy of the pruning step and the saliency based NMS, as used in [145] for fixed-scale keypoint detectors, we have named our final step as pruning step. But it can also be viewed as a relaxed NMS step as well. The reason for naming it as pruning step is that the output of this step is not always a set of distinctive keypoints and according to [145], NMS step is aimed at producing distinctive keypoints.

Pseudocode of the proposed salient region detection is presented in Algorithm 1, while Algorithm 2 presents for the pruning step. In Algorithm 2, $E_{3}$ represents the smallest eigenvalue, $g_{i}$ represents the nearest neighbours of $d_{i}, C_{d i}$ represents the covariance matrix of the points $d_{i}$ and $C_{g i}$ represents the covariance matrix of the points $g_{i}$. The source code of the proposed method is available at https://sites.google.com/site/honokeypointsets/

\subsection{Experimental Evaluation}

\subsubsection{Datasets}

We evaluate and compare the proposed keypoint detector with other state-of-the-art keypoint detectors using the standard 3D keypoint detection benchmark ${ }^{2}$ datasets provided by Tombari et al. [145]. They provide five datasets, namely, Kinect, SpaceTime, UWA, StanfordViews and Retrieval, the evaluation methodology and scripts to compare a new keypoint detector with existing ones on these datasets. Kinect and SpaceTime datasets have planar backgrounds present in the scene whereas other datasets do not have any background. Retrieval and StanfordViews are synthetic datasets and we have used the datasets with Gaussian noise of 0.1x mesh resolution for evaluation purposes.

\footnotetext{
${ }^{2}$ All the datasets and evaluation scripts are available at http://vision.deis.unibo.it/keypoints3d/?page_id=2
} 


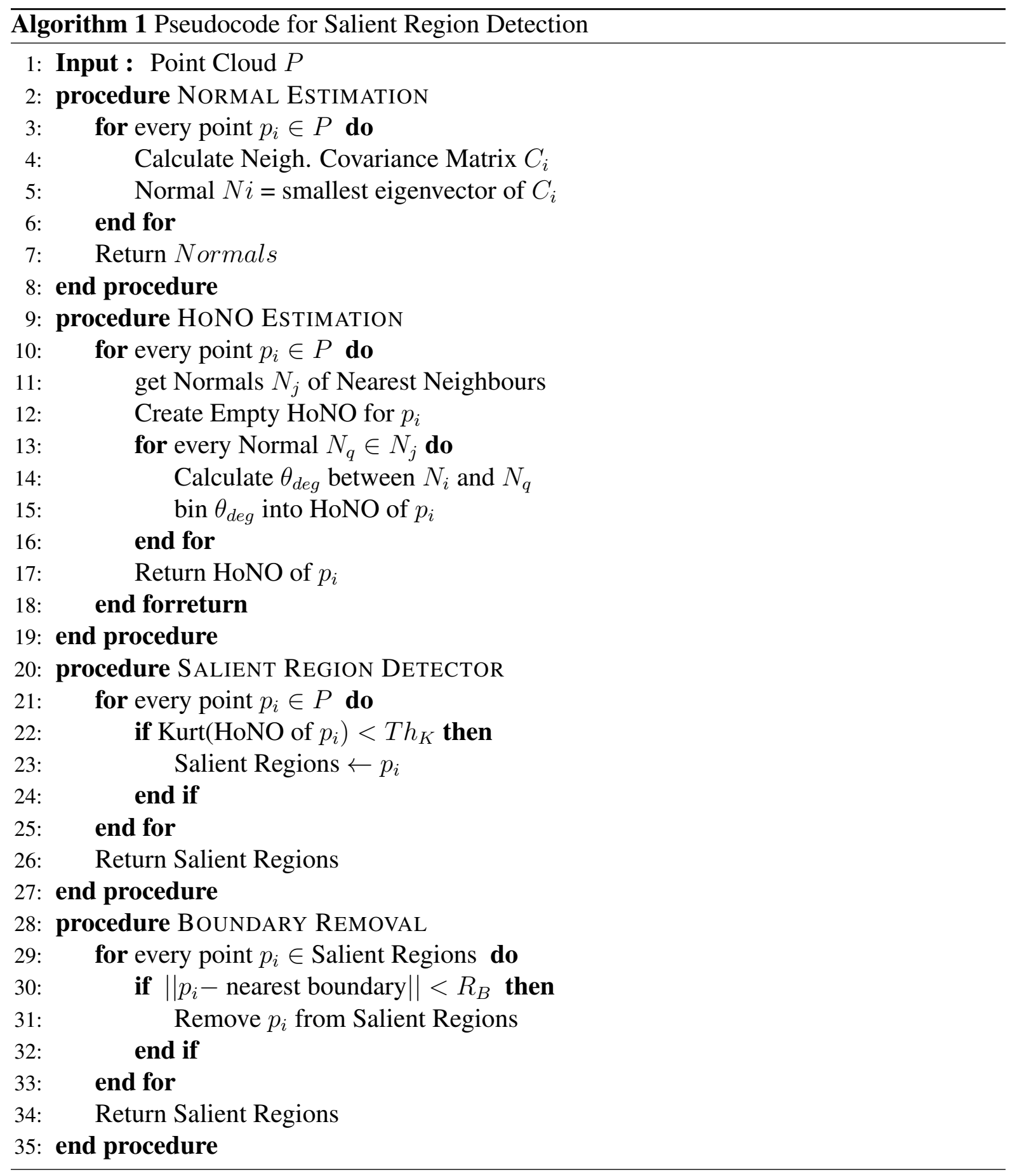

\subsubsection{Evaluation Metric: Repeatability}

An important characteristic of a keypoint detector is its repeatability, which evaluates if the keypoint detector is able to find the keypoints at the same locations on an object from different views, in the presence of background, partial occlusion, clutter, etc. To capture the repeatability of a keypoint detector, the following tests as mentioned in $([6,148,145])$ are performed. Lets consider a scene and an object model with known ground truth transformation between 


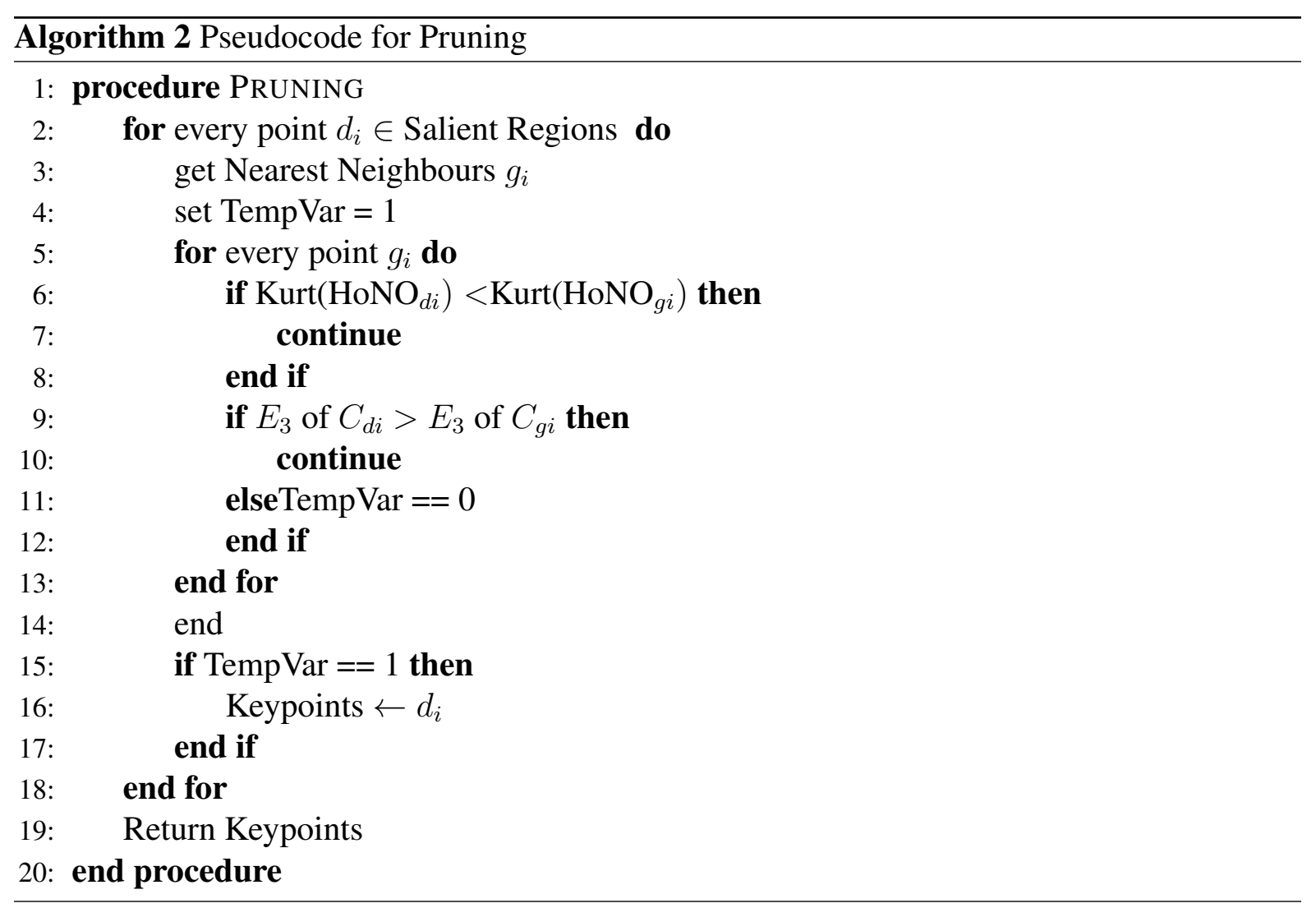

them. A keypoint detector under consideration detects keypoints on both scene and model. Absolute repeatability ${ }^{3}$ calculates the number of true keypoint matches between a scene and a model. Relative repeatability is obtained as the ratio of absolute repeatability and the number of keypoints detected on the model that are not occluded in the scene.

\subsubsection{Parameters}

All the radius parameters, namely, $R_{\text {normal }}, R_{H o N O}$ and $R_{d}$ are set to the same value as the scale used for experimentation. For boundary extraction, we approximately used $4 \mathrm{x}$ mesh resolution. Finally, $T h_{K}$, the threshold to detect salient regions is set to $6,13,8,12$ and 12 for Kinect, UWA, SpaceTime, Retrieval and StanfordViews datasets respectively. These values were chosen so as to detect similar number of keypoints as detected by other keypoint detectors in the comparative evaluation performed by Tombari et al. [145].

\footnotetext{
${ }^{3} \mathrm{~A}$ small note on calculating absolute and relative repeatability, one can also look at [145] for explanation. http://pointclouds.org/blog/gsoc12/gballin/repeatability.php
} 

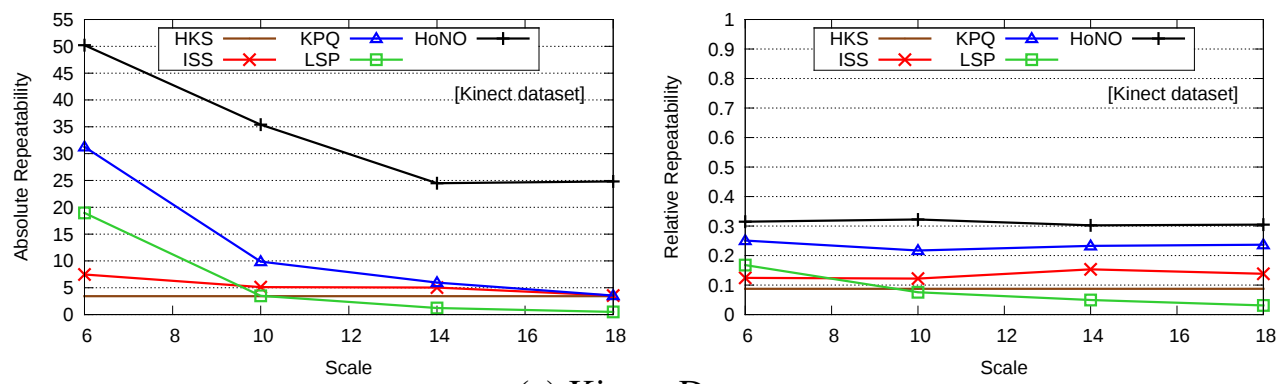

(a) Kinect Dataset
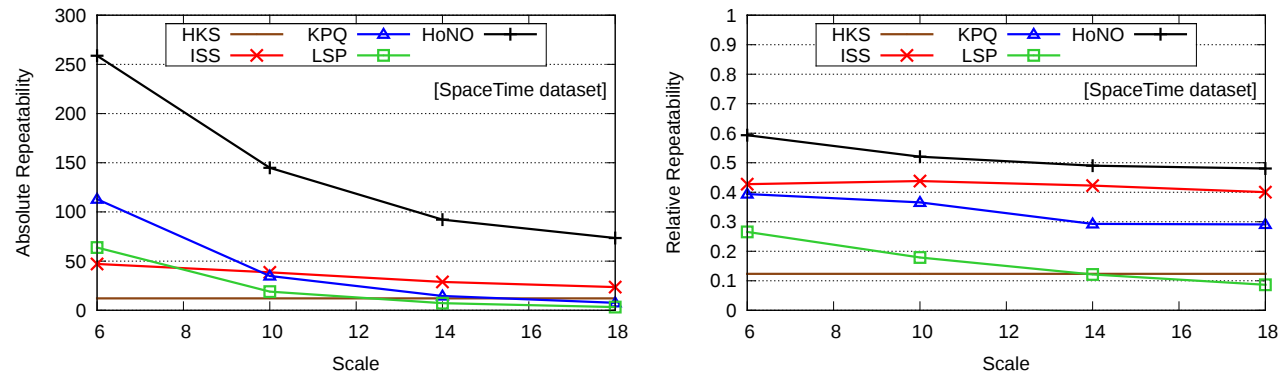

(b) SpaceTime Dataset
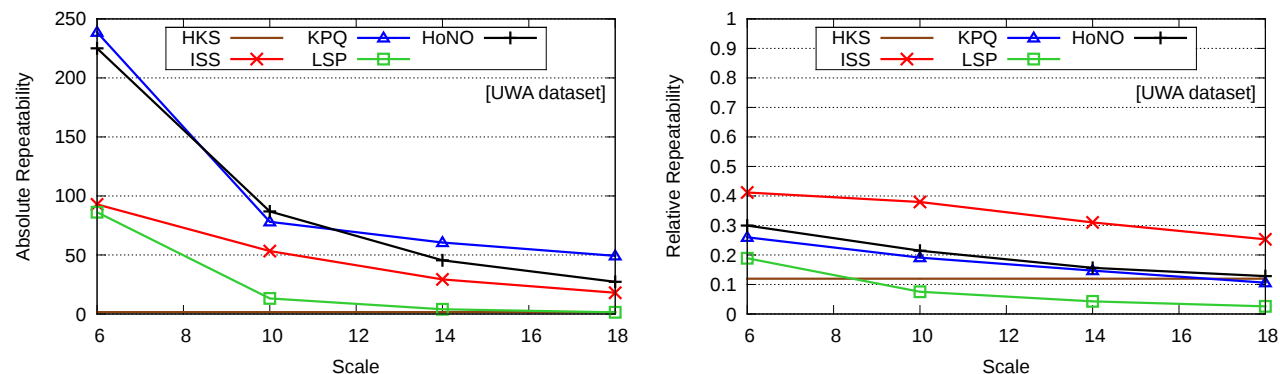

(c) UWA Dataset
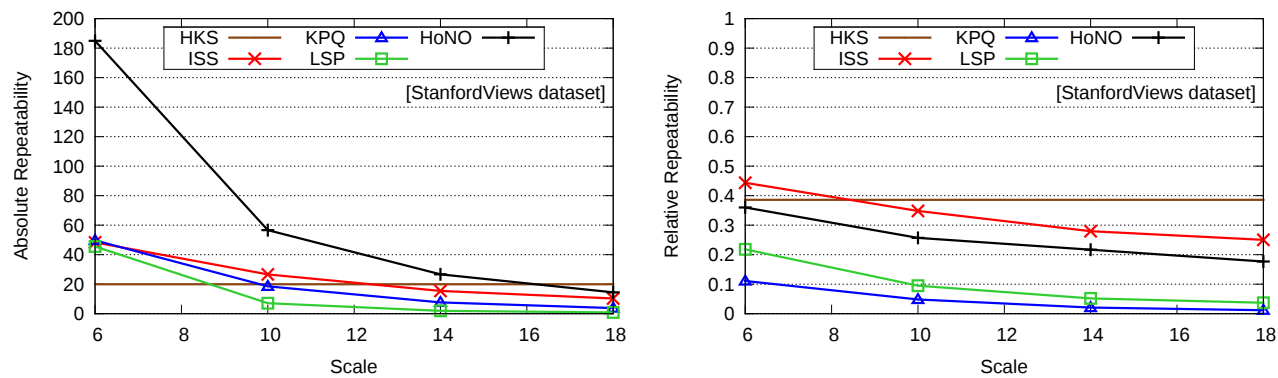

(d) StanfordViews Dataset
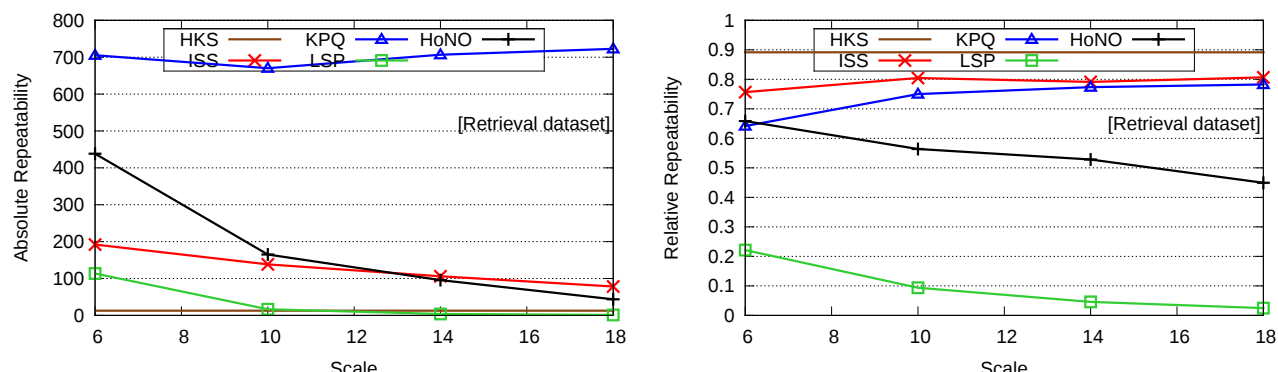

(e) Retrieval Dataset

Figure 3.2: Absolute and Relative Repeatability Tests on Kinect, SpaceTime, UWA, StanfordViews and Retreival datasets. 


\subsubsection{Repeatability Results}

Absolute and relative repeatability of the proposed keypoint detector, HoNO, in comparison with the existing ones, namely, ISS [162], LSP [25], KPQ [96] and HKS [140] keypoint detectors on these five datasets is shown in Fig. 3.2.

- It can be seen from Fig. 3.2(a) and Fig. 3.2(b) that HoNO outperforms other keypoint detectors, by offering high absolute and relative repeatability, on both Kinect and SpaceTime datasets.

- On UWA dataset, HoNO offers similar performance as KPQ and second-best performance for relative repeatability after ISS, as can be seen from Fig. 3.2(c).

- Fig. 3.2(d) and Fig. 3.2(e) show that HoNO keypoints offer second best relative repeatability on StanfordViews and third best on Retrieval datasets.

- ISS and KPQ keypoint detectors may seem to perform well on UWA, StanfordViews and Retrieval datasets. But they have a serious drawback of detecting keypoints on planar regions as explained below.

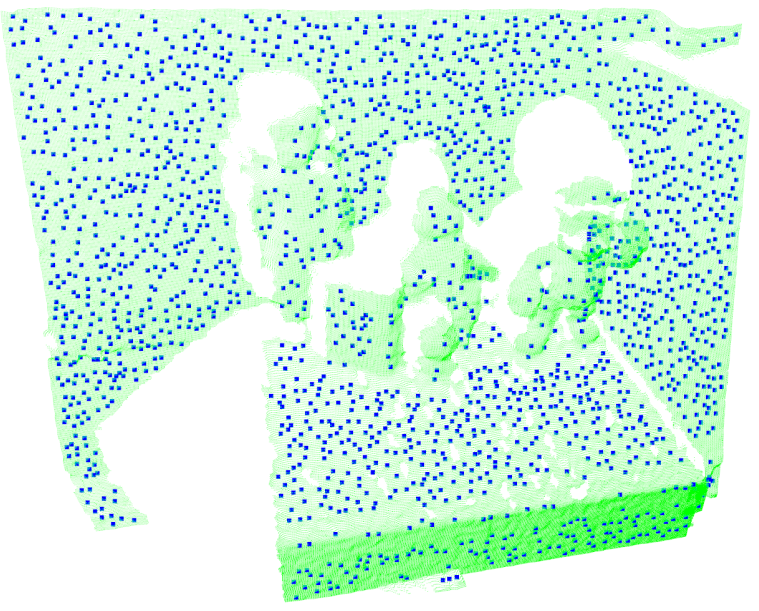

(a) ISS Keypoints $\left(T h_{21}=T h_{32}=0.975\right)$

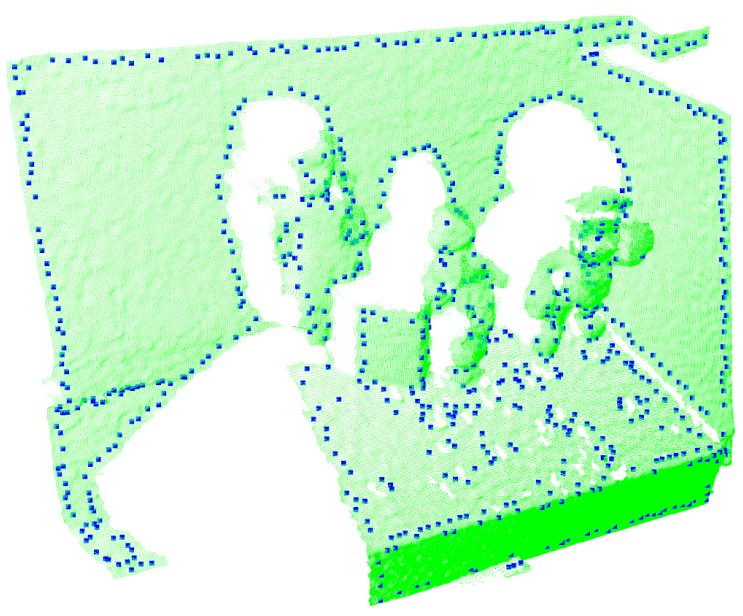

(b) ISS Keypoints $\left(T h_{21}=T h_{32}=0.75\right)$

Figure 3.3: ISS keypoints detected on scene_0 05 ( from Fig. 3.1 ) with two different settings of $T h_{32}$ and $T h_{21}$ parameters. 


\subsubsection{Drawback of ISS and KPQ keypoint detectors}

ISS keypoint detector calculates the eigenvalues $E_{1}, E_{2}$ and $E_{3}$ (in decreasing order) of the neighbourhood covariance matrix $C$ for every point $p_{i} \in P$, where $P$ is the input point cloud. Then it checks if $\frac{E_{2}}{E_{1}}<T h_{21}$ and $\frac{E_{3}}{E_{2}}<T h_{32}$ for pruning and the NMS is performed based on the smallest eigenvalue $E_{3}$. It can be noticed that $T h_{21}$ and $T h_{32}$ are the parameters that influence the keypoint positions. In Fig. 3.3, we illustrate the ISS keypoints detected with two different parameter settings on the scene scene_0 05 (shown in Fig. 3.1) from Kinect dataset.

ISS keypoints detected on scene_005 with $T h_{21}=T h_{32}=0.975$, are shown in Fig. 3.3(a). It can be noticed that the keypoints are evenly spread all over the scene and even lie on the planar background. This is because, the used parameters for comparative evaluation in [145], $T h_{21}=T h_{32}=0.975$, do not prune planar/less informative areas and the final keypoints are detected by NMS step alone. ISS keypoint detector with $T h_{21}=T h_{32}=0.975$ parameter setting, labels the considered point as a keypoint if it has the strongest normal in a radius of $R_{n m s}$, which might sometimes arise due to noise. Hence, ISS keypoints detected with these parameters can lie on planar regions, which is not desirable, because feature descriptor based keypoint matching would become highly ambiguous.

We experimented by decreasing the value of $T h_{21}$ and $T h_{32}$ parameters of ISS keypoint detector and it was observed that with proper parameters, planar regions can be avoided and keypoints lie in more informative areas. ISS keypoints detected on scene scene_005 with $T h_{21}=T h_{32}=0.75$ are shown in Fig. 3.3(b). It can be seen that the detected keypoints do not lie on planar regions as they are discarded in the pruning step. However, most of the remaining keypoints, as can be seen from Fig. 3.3(b), lie near boundaries which is not desirable. We evaluated the ISS keypoint detector on UWA dataset by slowly decreasing the thresholds $T h_{21}$ $=T h_{32}=[1,0.9,0.8,0.7]$, and the relative repeatability in comparison with HoNO keypoint detector is shown in Fig. 3.5. Setting the thresholds $T h_{21}$ and $T h_{32}$ to 1 means that there is no pruning at all and the keypoints are detected by NMS step alone. It can be noticed from Fig. 3.5 that there is significant decrease when $T h_{32}$ and $T h_{21}$ are changed from 1 to 0.9 and HoNO keypoint detector outperforms ISS in terms of relative repeatability. Hence, it can be claimed from Fig. 3.3(a), Fig. 3.3(b) and Fig. 3.5 that HoNO keypoints are more meaningful and offer better repeatability than ISS keypoints.

Even the KPQ keypoint detector has the same problem of detecting keypoints on planar regions as it employs similar pruning method ([145]) as in ISS and because of the parameters used in evaluation. One can see that KPQ keypoints also lie on planar regions as shown in Fig. 8 of Tombari et al. [145]. We reproduced the Fig. 8 from the paper [145], here in Fig. 3.4. 


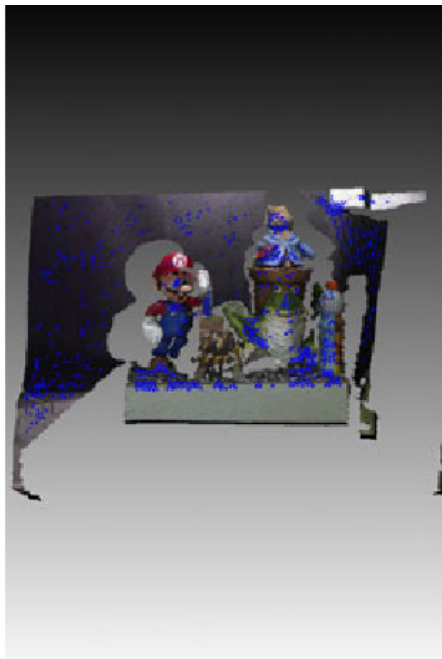

(a) without border points

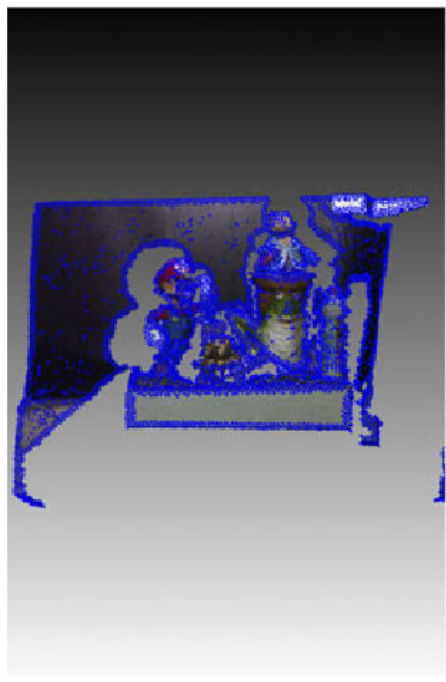

(c) with border points

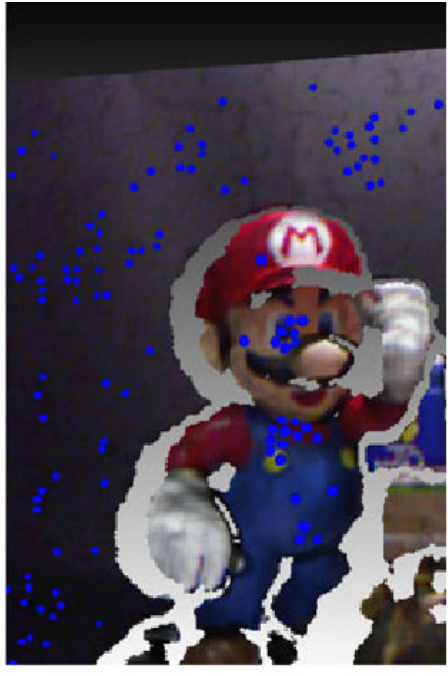

(b) without border points

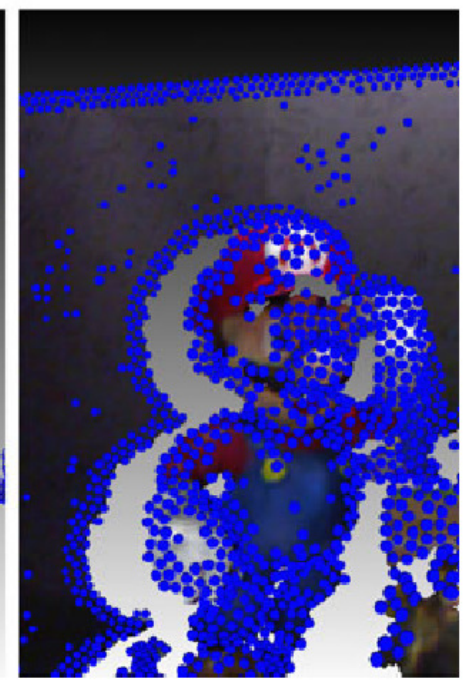

(d) with border points

Figure 3.4: KPQ keypoints detected on a scene from Kinect dataset. This figure is reproduced from Fig. 8 of Tombari et al. [145]. This shows that even KPQ keypoint detector has the drawback of detecting keypoint on planar regions (blue keypoints on black planar backgrounds), which is not desirable because 3D feature descriptors can not find any meaningful keypoint matches in such scenarios.

In short, ISS and KPQ keypoint detectors with the employed parameters in [145], whose repeatability is shown in Fig. 3.2, do not detect meaningful and useful keypoints as most of them lie on planar regions. Moreover, by changing the parameters to detect informative keypoints on curved regions, there is significant drop in their performance and offer lower repeatability than the proposed HoNO keypoints. 


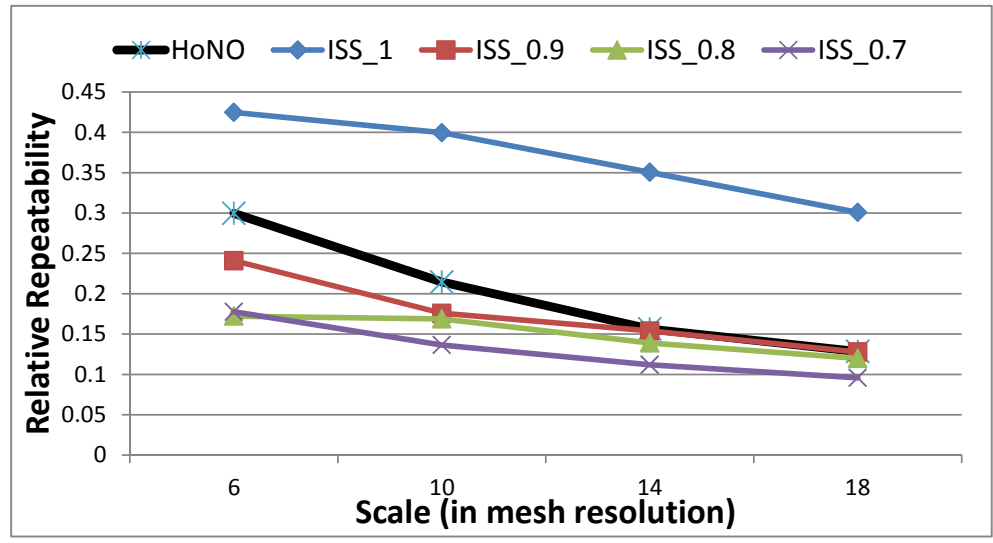

Figure 3.5: Performance of ISS keypoint detector with varying $T h_{32}$ and $T h_{21}$ in comparison with HoNO. ISS_0.9 represents that $T h_{32}=T h_{21}=0.9$.

\subsubsection{Other Keypoint Detectors}

The HKS keypoint detector offered good relative repeatability on StandfordViews and Retrieval datasets but its absolute repeatability highlights that it detects less number of keypoints and do not perform well on Kinect and SpaceTime datasets. As mentioned in [145], on a computer with 6 GB RAM, HKS can only run on meshes with a maximum of 30,000 vertices, which highlights its need for high computational resources.

SURE, NARF and SDTP keypoint detectors detect much lesser number of keypoints and their repeatability on these datasets is low. We tested with various parameters and it turned out that SURE keypoint detector detects $\sim 100$ keypoints on scenes from Kinect dataset and has relative repatability less than 0.1. Wang et al. [153] have shown that SDTP outperforms NARF in relative repeatability on Kinect and SpaceTime datasets. However, SDTP's realtive repeatability is still lower than the proposed HoNO keypoint detector, which can be seen by from their published results [153] on Kinect and SpaceTime datasets.

One question may arise in the reader's mind that the proposed approach may detect more number of keypoints when compared to others and that might be the reason for high performance in repeatability tests. We address this question by quantitatively showing in Fig. 3.6 that our method detects almost the same number or some times lesser number of keypoints than other keypoint detectors. Specifically, in Fig. 3.6, we show the number of keypoints detected on a scene0 05 from Kinect dataset and their variation with the scale.

In any real world application, it is highly possible to have scenes with some kind of planar background. The keypoint detectors should not detect keypoints on planar surfaces as feature descriptor based keypoint matching would become highly ambiguous or in other words, 3D feature descriptors can not find and encode any distinctive information from keypoints on planar 


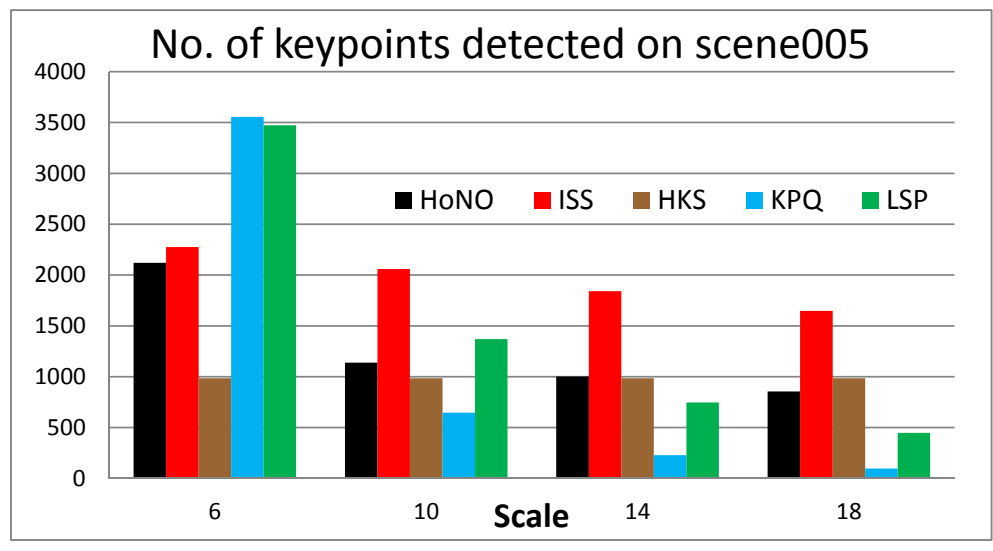

Figure 3.6: Number of keypoints detected on scene005 (shown in Fig. 3.1) by different keypoint detectors and their variation with scale.

regions, hence they can not find any keypoint correspondences. From the datasets provided by Tombari et al. [145], Kinect dataset is closer to real world applications as it has noise that arises directly from Kinect sensor and planar backgrounds that are quite common. The proposed HoNO keypoint detector outperforms other keypoint detectors on this dataset. Moreover it can be claimed from Fig. 3.2, Fig. 3.3, Fig. 3.4 and Fig. 3.5 that HoNO offers more salient, reliable and repeatable keypoints than other existing keypoint detectors.

\subsubsection{Computational Requirements}

The proposed method takes around 6-8 seconds to detect keypoints on a Kinect point cloud, which contains about 3 million points. The results from the seminal work by Tombari et al. (2013), which compared various 3D keypoint detectors showed that other 3D keypoint detectors take around 5 seconds on a point cloud with 1 million points. Hence in terms of computation, our approach is comparable or even better than other works. However, as we did not implement the other keypoint detection algorithms that we compared with, on our system, and as the system configuration can affect the computational requirements, we refrain from direct comparison, as it would not be fair.

We compared our proposed keypoint detection algorithm with other methods based on the datasets and evaluation scripts provided by Tombari et al. (2013). These evaluation scripts makes it easier to compare a new keypoint detection method directly with existing works, without any need to implement all the methods and vary their parameters. The source code of the proposed method is available at https ://sites.google.com/site/honokeypointsets/. The provided implementation can be further optimized by parallelizing the HoNO estimation for every point in the point cloud, as these HoNO computations are independent. This can 


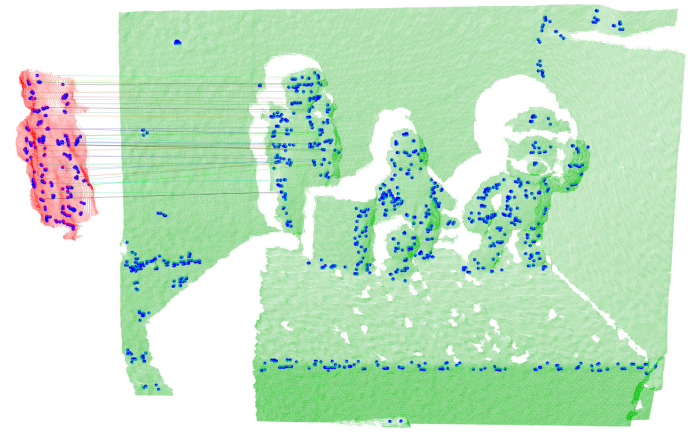

(a) Object: Dol1018 and Scene: scene_005

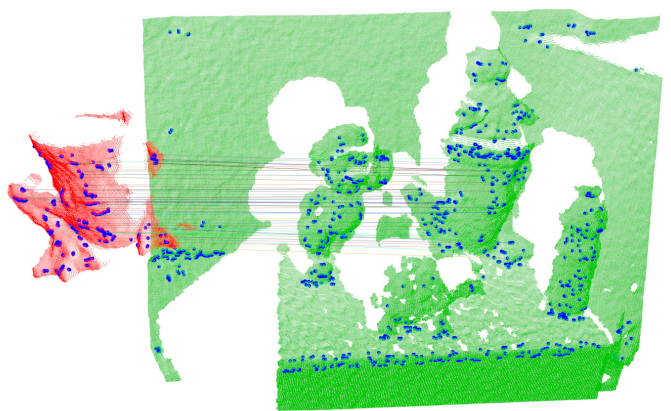

(c) Object: Frog013 and Scene: scene_019

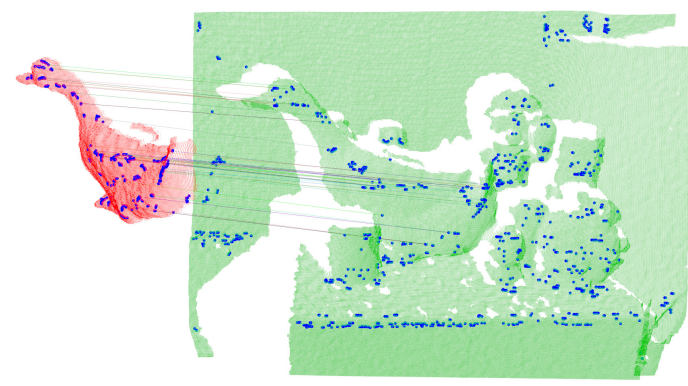

(b) Object: Duck009 and Scene: scene_014

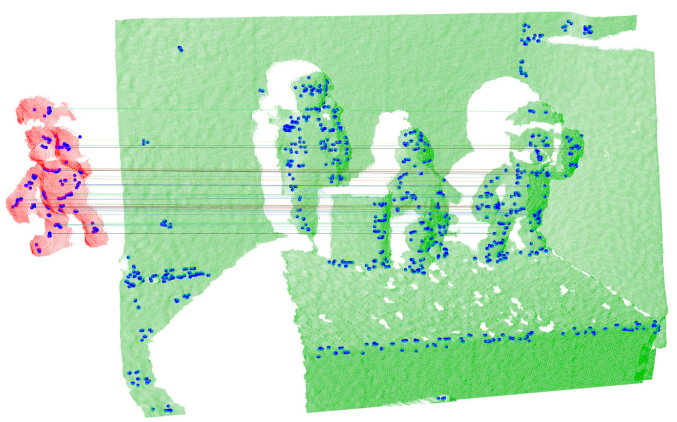

(d) Object: Mario000 and Scene: scene_005

Figure 3.7: HoNO keypoints are detected on Doll, Duck, Frog and Mario object models from the Kinect dataset and they are matched with SHOT feature descriptor to the keypoints detected in various scenes. In the figure, object models are shown in red, scenes are shown in green while the HoNO keypoints are shown in blue.

further reduce the computational time. Finally, we would like to emphasize that the main aim of this work is to propose a simplistic yet effective keypoint detection method that offers better repeatability.

\subsubsection{HoNO Keypoint sets + SHOT Feature Descriptor}

Finally, we combine HoNO keypoint sets with the SHOT [128] feature descriptor and illustrate few results on the Kinect dataset in Fig. 3.7. We consider various object models from the Kinect dataset such as Doll, Duck, Frog and Mario and scenes that contain them. HoNO keypoints are detected with a scale of $10 \times$ mesh resolution $\approx 0.02 m$, which are then matched with SHOT feature descriptors constructed with a support size of $0.08 m$ and RANSAC [53] was used to remove false correspondences. It can be seen from Fig. 3.7 that the keypoints from the model are matched with the relevant keypoints in the scene successfully. Once again, it can be seen from Fig. 3.7 that the detected HoNO keypoints do not lie on planar regions, but only lie on curved and informative regions of the scene point cloud. 


\subsection{Conclusion}

In this chapter, a new method to detect 3D keypoint sets on point clouds was proposed by leveraging on histogram of normal orientations (HoNO) that is calculated at every point. The proposed keypoint detection method independently tackles each of the steps, salient region detection and pruning, based on HoNO and local properties of the neighbourhood covariance matrix. Compared to existing 3D keypoint detectors, the detected keypoints are highly repeatable, avoid planar regions effectively and lie on informative regions of 3D point clouds. The detected keypoints lie in groups and these groups are distinctive.

The detected keypoints essentially represent informative 'places' and 'points' present in the scene. This opens a new research direction that aims at establishing accurate 'place' matches by developing feature descriptors that employ information from neighbouring keypoint sets.

While in this chapter, we proposed a generic 3D keypoint detector that offers high repeatability and salient keypoint sets, in the next chapter, we consider a specific 3D perception application and propose a new keypoint detection module based on its requirements. 


\section{Chapter 4}

\section{Sparse Depth Odometry: Keypoint based Pose Estimation from Dense Depth Data}

\subsection{Introduction}

Specifically, considering a 3D perception application, Simultaneous Localization and Mapping (SLAM), which requires ego-motion estimation of a camera as it moves in the environment, RGB-D cameras have helped in solving the scale ambiguity which is imminent with monocular cameras. With the availability of RGB-D cameras, various algorithms were proposed to perform SLAM, which are reviewed in Chapter 2. Existing SLAM proposals with RGB-D cameras can be classified into two categories, firstly $R G B+$ Depth methods and secondly, Depth-alone methods. $R G B+$ Depth methods employ both RGB and depth information for ego-motion estimation while Depth-alone methods rely only on depth information alone.

Motivation: Depth-alone methods have an edge over RGB+Depth methods because they do not require the environment to be illuminated. Moreover, Depth-alone methods can be combined with $R G B+$ Depth to make them fail-proof in low lighting conditions and when there is no texture in the scene. However, all the existing Depth-alone methods fall in the Dense methods category as explained in Sec. 2.4 of Chapter 2. Essentially, existing Dense Depth-alone methods employ all the depth information to estimate the ego-motion, employ memory and computationally intensive truncated signed distance function (TSDF), rendering them impractical to work in real time without a GPU.

Hence, in contrast to existing Depth-alone methods which are all Dense methods, we propose a Sparse Depth-alone method, which we call as Sparse Depth Odometry (SDO). Sparse Methods as mentioned in Sec. 2.4 capitalize on extracting keypoints from the input data, thus all the consecutive steps in the algorithm just work on few hundreds to thousands of keypoints, rather than working with millions of raw points. Hence, the task boils down to extracting 
keypoints that represent the scene comprehensively, which work well with rest of the steps in SDO.

Contribution: In this chapter, we consider a specific 3D perception application, SLAM with an RGB-D camera and provide a solution, Sparse Depth Odometry (SDO). SDO differs from the existing Depth-alone odometry methods as it does not employ memory and computationally intensive truncated signed distance function (TSDF), and hence SDO can run in real time without a GPU. At its core, SDO is powered by a new 3D keypoint detection module that represents the scene comprehensively to enable efficient ego-motion estimation. The proposed new 3D keypoint module comprises of two existing 3D keypoint detectors, SURE [51] and [134]. We show both theoretically and experimentally that the combination of these two keypoint detectors, represents the scene comprehensively and works best with SDO. From top view, our contribution is the proposal of a Sparse Depth-alone odometry method SDO, and the actual novelty lies in the proposal of a new keypoint detection module.

\section{Why can't we use an existing keypoint detector that offers high repeatability ?}

A point to note is that the detected keypoints play a crucial role in pose estimation accuracy, and selecting an appropriate keypoint detector is not a trivial task. This is because the existing 3D keypoint performance evaluation methods Tombari et. al [147], namely the absolute repeatability test and relative repeatability test, assume that the transformation between the scene and the model is known and then try to find the number of keypoint matches, whereas in pose estimation applications, such as SDO, the aim is to find the accurate transformation between two point clouds with uncertain keypoint matches. In repeatability tests, model comprises of the object point cloud alone, without any background, while scene has various objects and a background. But this is not the case with pose estimation between two frames as both frames will have a background and the outliers that may arise from the background should be considered for evaluation. Hence, a new approach that has visual, theoretical and experimental analysis of keypoint neighbourhoods and their performance with SDO has been used here to evaluate various keypoint detectors. This extensive analysis has led to the development of a new 3D keypoint detection module that employs a combination of two keypoint detectors and finds reliable keypoints that offer better performance with SDO than other keypoint detectors.

\section{Is it possible to employ HoNO based keypoint detection method for SDO ?}

It is not feasible to employ HoNO based keypoint detection algorithm for SDO because, firstly it detects keypoint sets and not distinctive keypoints, secondly, HoNO keypoint detection does not work real time, and finally, the crucial aspect of SDO is that, we do not employ 3D feature 
descriptors to find keypoint matches but assign nearest neighbours as the keypoint matches to initialize ICP algorithm. Therefore, HoNO keypoint sets is not suitable for SDO.

Following in this chapter, we first explain the complete pipeline of SDO in Sec. 4.2. Second, we present the analysis performed on various keypoint detectors to design the new keypoint detection module and present the reasons for selecting SURE and NARF keypoint detectors in Sec. 4.3. Finally, an extensive evaluation of SDO with the proposed keypoint detection module on publicly available datasets is presented and compared with state-of-the-art odometry methods in Sec. 4.4.

\subsection{Sparse Depth Odometry}

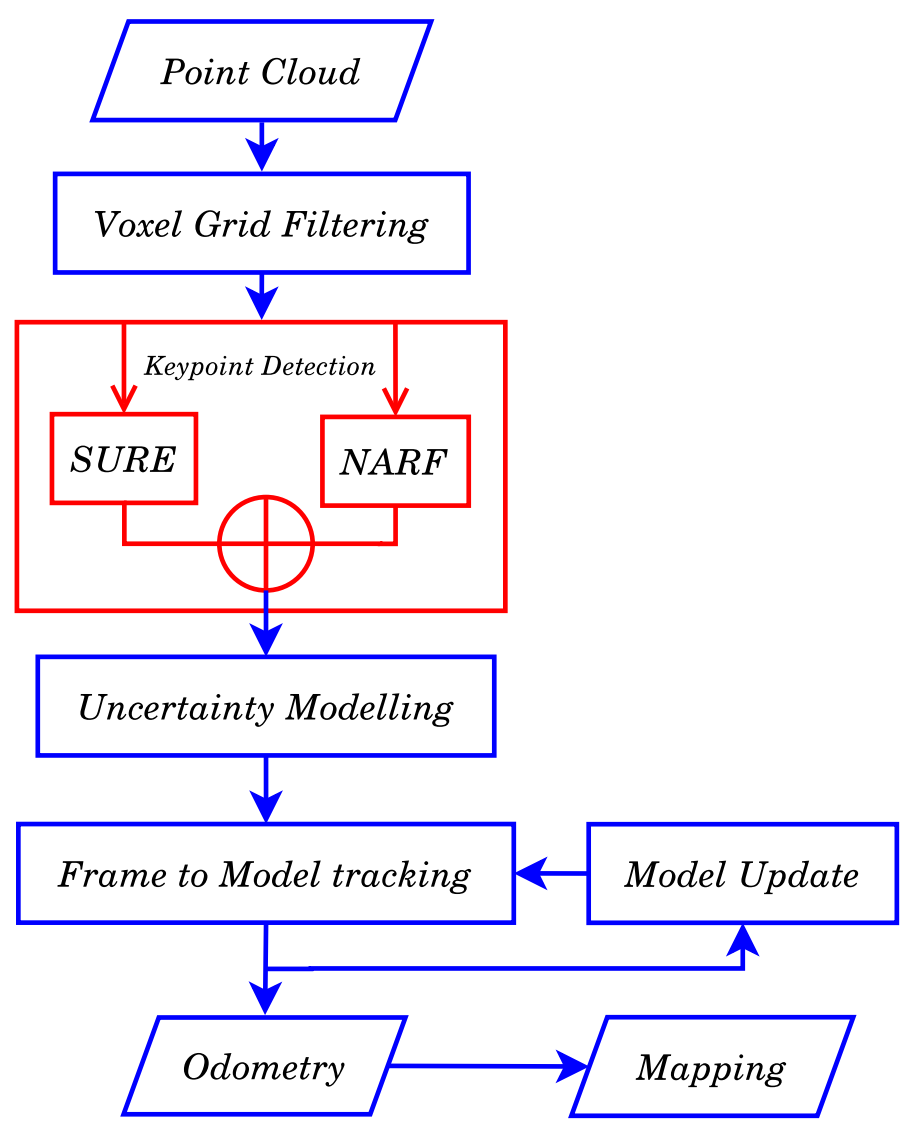

Figure 4.1: Sparse Depth Odometry

SDO, as illustrated in Fig. 4.1, down samples the input point cloud, finds keypoints, models their position uncertainty, estimates the odometry with a frame to model based registration and creates a 3D map. 


\subsubsection{Voxel Grid Filtering}

A voxel grid filter approximates all points in a voxel with its centroid, hence decreasing the point cloud size considerably, while effectively removing some noise. A voxel grid filter with a leaf size of 0.01 metres was used in our implementation, which played a key role in reducing computational requirements in the following steps of SDO, hence making it real time.

\subsubsection{Keypoint detection}

The proposed keypoint detection module comprises of SURE [51] and NARF [134] keypoint detectors. It concatenates the keypoints from both of them to form a final set of keypoints. Our main contribution lies in the way we select these two keypoint detectors from the available ones and concatenate them to achieve better performance with SDO. The theoretical and experimental analysis performed to design the new keypoint detection module is explained in Sec. 4.3.

\subsubsection{Uncertainty Modelling of 3D Keypoints}

As detected keypoints on depth data from Kinect-style sensors are noisy, we follow [37] and model the varying positions of 3D keypoints as multi-variate Gaussian distributions.

Depth cameras like Microsoft Kinect and Asus Xtion Pro Live provide depth images, which can be converted to $3 \mathrm{D}$ point clouds of the form $[x, y, z]$ in the camera coordinate frame using standard mathematical model of a depth camera as explained in detail by Khoshelham and Elberink [80] or succintly by Dryanovski et al. [37]. The depth measurement $z$ estimated by Kinect-like depth cameras has a tolerance of $0.002 \mathrm{~m}$ at $1 \mathrm{~m}$ distance to $0.07 \mathrm{~m}$ at $5 \mathrm{~m}$ distance. Khoshelham and Elberink [80] estimated the uncertainty in $z$ by considering $d$, disparity in image space, as a random variable with standard deviation $\sigma_{d}$. The standard deviation $\left(\sigma_{z}\right)$ of $z$ from the standard camera model [37] turns out to be

$$
\sigma_{z}=\frac{1}{f b}\left(\sigma_{d} \mu_{z}^{2}\right)
$$

where $b$ is the base length between the IR camera and IR projector and $f$ is the focal length of the IR camera. The value of $\sigma_{z}$ using the calibrated parameters was found by Khoshelham and Elberink [80] to be $1.45 * 10^{-3} \mu_{z}^{2}$. Each keypoint's $z$ coordinate is modelled as a Gaussian distribution with the depth value from the sensor as its mean and estimated $\sigma_{z}$ as its standard deviation. Finally every keypoint $[x, y, z]$ is modelled as a multivariate Gaussian distribution with mean $\boldsymbol{\mu}=\left[\mu_{x}, \mu_{y}, \mu_{z}\right]$ and covariance matrix $\sum$ (Please refer to [37] for equations to calculate 
covariance matrix). This modelling of keypoints as Gaussian distributions is an approximation as there can be some position uncertainty induced by the keypoint detection process, which is not considered in this current modelling and this can be a reason that simple Gaussian model offers higher accuracy than the Gaussian kernel proposed in [37].

\subsubsection{Frame to Model based Tracking}

This module estimates the pose of the depth camera incrementally by registering the keypoints on the current point cloud with a model of keypoints estimated from the previous point clouds. We employ a frame to model based registration framework, as Newcombe et al. [103] and Dryanovski et al. [37] have demonstrated in their works that frame to model based tracking offers higher accuracy than frame to frame matching.

Let's call the keypoints on the current point cloud as data $D$ and the keypoints from past frames as model $M$. Keypoints from the data $D$ can be registered with the model $M$ and relative transformation can be estimated in the following different ways.

First Compute and match the feature descriptors of keypoints in $D$ and $M$ and estimate the transformation accordingly.

Second Nearest neighbor based correspondence estimation, with false correspondence rejection using RANSAC and transformation estimation using SVD (singular value decomposition) or the LM (Levenberg-Marquardt) method.

Third Simple nearest neighbor association and iterative transformation estimation using SVD.

Fourth Semi-probabilistic nearest neighbor correspondences and transformation estimation using SVD, as mentioned by [37].

The first option was left out as it is time consuming to compute and match the $3 \mathrm{D}$ feature descriptors in their high dimensional spaces and remove the outliers. The second option mentioned above was implemented using the Point Cloud Library [126]. In the third option, a kd-tree representation of the model $M$ was created with nearest neighbours as associations and an SVD based method [10] was employed to estimate the transformation iteratively. The fourth option is a semi-probabilistic way to do correspondence estimation. In this method, a kd-tree representation of the model $M$ is created. For every key point in the data $D, k$ nearest neighbours from the model $M$ are found by Euclidean distance measurement. The one with the least Mahalanobis distance in the $k$ nearest neighbours is considered as a correspondence 
if the distance between them is lower than a threshold. The transformation is estimated by an SVD based method [10] iteratively with the found correspondences. We have tested the accuracy of SDO with the second, the third and the fourth options on TUM-RGBD datasets with ground truths. The third option out-performed the others in terms of computational efficiency and accurate pose estimation.

\subsubsection{Model Update}

As discussed before, the correspondences are estimated between data $D$ and model $M$ using the simple nearest neighbour approach and the transformation is estimated using SVD. This module of updating the model of keypoints by a Kalman filter is inspired from Dryanovski et al. [37]. Initially, the model $M$ is empty and keypoints are added to it in the first iteration. From the second iteration on-wards, the key points in the model $M$ are updated using Kalman filter framework with data $D$ as the observation. In the Kalman filter, the predicted position of a key point is its current position while the observation is the estimated correspondence. If there is no correspondence then it is added as a new point into the model $M$. The model $M$ has a fixed size, thus when the model is full, the older keypoints are replaced by the newer ones.

\subsubsection{Mapping}

Keyframes are extracted for a certain change in the estimated pose of the sensor. These keyframe point clouds, along with their poses, are processed by OctoMap [74] library to create a dense 3D voxel map of the environment. The resultant OctoMap representations of various scenes are shown later.

\subsection{On Selecting SURE and NARF Keypoint detectors}

The keypoint detection module plays a vital role in SDO as it condenses an input point cloud to a small number of keypoints. As mentioned in Section 4.1, repeatability tests based evaluation may not highlight which keypoint detector works well for pose estimation applications such as SDO. Hence we performed a series of tests on various keypoint detectors and finally designed a keypoint detection module that offers reliable keypoints and represents the scene holistically.

We propose and perform two tests that evaluate various keypoint detectors on two important characteristics. Firstly, the keypoints should have neighbourhoods with high variance thus coming from regions that have high surface variations. Secondly, the detected keypoints should 
represent the scene comprehensively. In order to find a keypoint detector that has the desired properties, two tests that quantify the variance and the structure of the keypoint's neighbourhood are designed and described below.

- The Neighbourhood Variance Test calculates the variance of the neighbourhood of the detected keypoints; i.e., it checks if the keypoints come from a varying neighbourhood.

- The Neighbourhood Structure Test determines if the neighbourhood of the keypoint is planar, spherical or in the shape of a spindle.

\subsubsection{Neighbourhood Variance Test}

This test quantifies the variance of the keypoint neighbourhoods in a scene. The inputs for this test are the point cloud of the scene and the keypoints detected on it by a specific keypoint detector. For every keypoint, a covariance matrix is estimated from the sample points that lie in its neighbourhood of radius $r$. This value of the radius $r$ is set to be the same as the support radius that the keypoint detector uses to find the keypoints in the scene. Once the covariance matrix of every keypoint in the scene is calculated, the trace of each covariance matrix is summed up and divided by the number of keypoints detected in that specific scene. In short, this test provides an estimate of the average variance of the neighbourhood of the keypoints detected by a keypoint detector in a specific scene.

\subsubsection{Neighbourhood Structure Test}

This test classifies the keypoints based on the structure of their neighbourhoods into either spindle, planar or spherical shaped ones. It is performed based on the eigen values of a keypoint's neighbourhood covariance matrix. Let the estimated eigen values be $\lambda_{1}, \lambda_{2}$ and $\lambda_{3}$, where $\lambda_{1}$ $\leq \lambda_{2} \leq \lambda_{3}$. The shape of the covariance matrix can be classified as

- Spindle shaped if $\frac{\lambda_{2}}{\lambda_{3}} \leq T_{e}$;

- Planar shaped if it's not spindle shaped and $\frac{\lambda_{1}}{\lambda_{2}} \leq T_{e}$; and

- Spherical shaped if its neither spindle shaped nor planar shaped,

where $T_{e}$ was set to 0.10 in all our experiments.

We considered five publicly available keypoint detectors namely, SURE [51], NARF [134], ISS [162], SIFT [126] and HARRIS [126], for comparison. More test results and the parameters used for evaluation can be found at https://sites.google.com/site/3dkeypoints/. In order to 


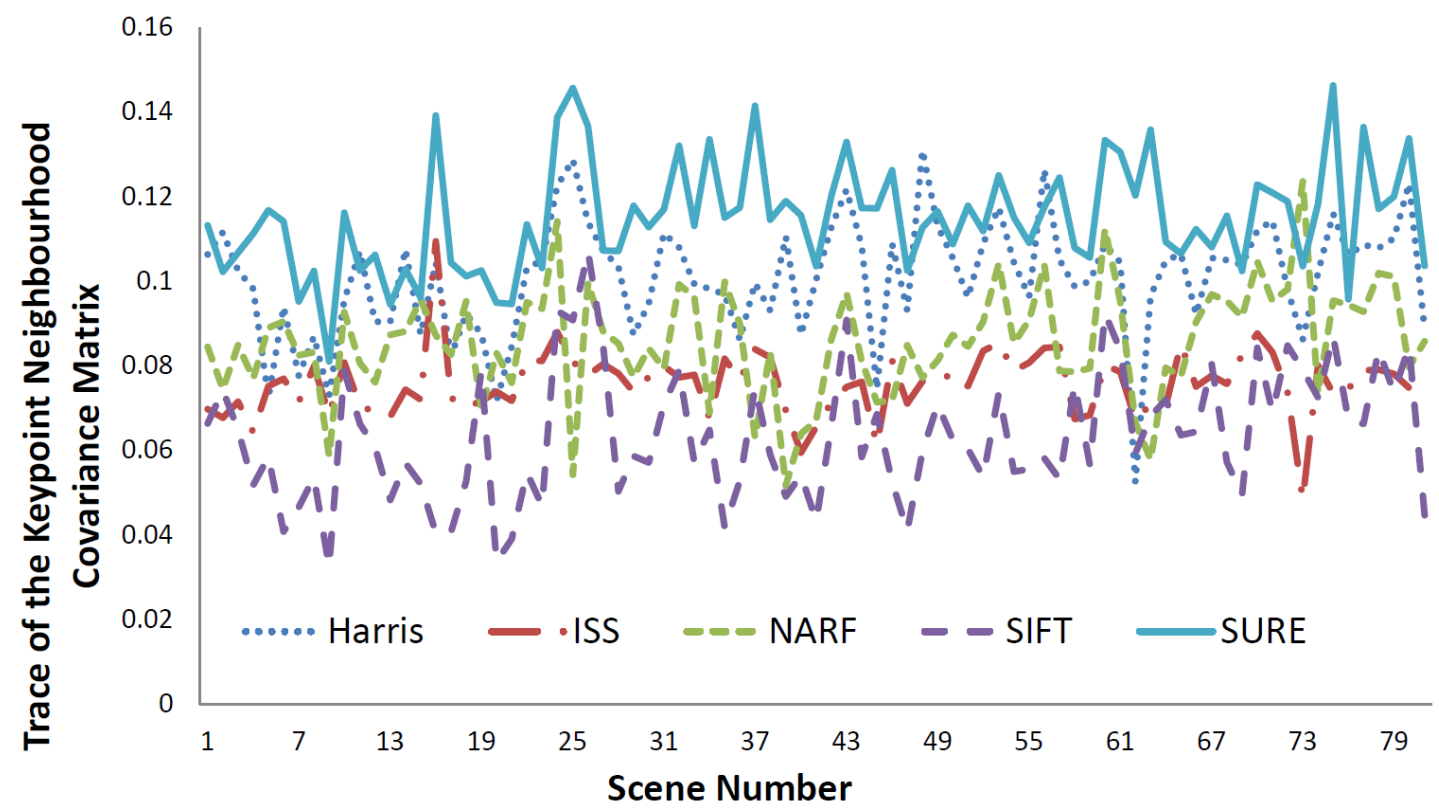

Figure 4.2: Neighbourhood Variance Test on 80 different scenes. It shows the average variance of a keypoint neighbourhood detected by various keypoint detectors in each of the 80 scenes.

evaluate the 3D keypoint detectors, 80 scenes from typical indoor environments, such as office, home, lab and workshop, were captured. On each scene, the keypoints detected by various keypoint detectors are classified into one of the three classes, as mentioned under Neighbourhood Structure Test and the average variance of the keypoints detected by a keypoint detector is calculated, as described under Neighbourhood Variance Test.

\subsubsection{Observations}

- The results from the Neighbourhood Variance Test shown in Fig. 4.2 highlight that the SURE [51] keypoint detector offers keypoints whose neighbourhoods have higher variance compared to the other keypoint detectors.

- From the Neighbourhood Structure Test, it is observed that all the keypoint detectors offer zero or a negligible number of keypoints from spindle neighbourhoods. Next, almost all of the SURE keypoints are from spherical neighbourhoods. As shown in Fig. 4.3, the NARF [134] keypoint detector offers the highest ratio of planar keypoints to total number of keypoints when compared to the other keypoint detectors. Different neighbourhood structures essentially means that the keypoints come from various parts of the scene.

- Hence in order to have keypoints that come from varying neighbourhoods and describe the scene comprehensively, a combination of the SURE and NARF keypoint detectors turns out to be a better choice than selecting only one keypoint detector. 


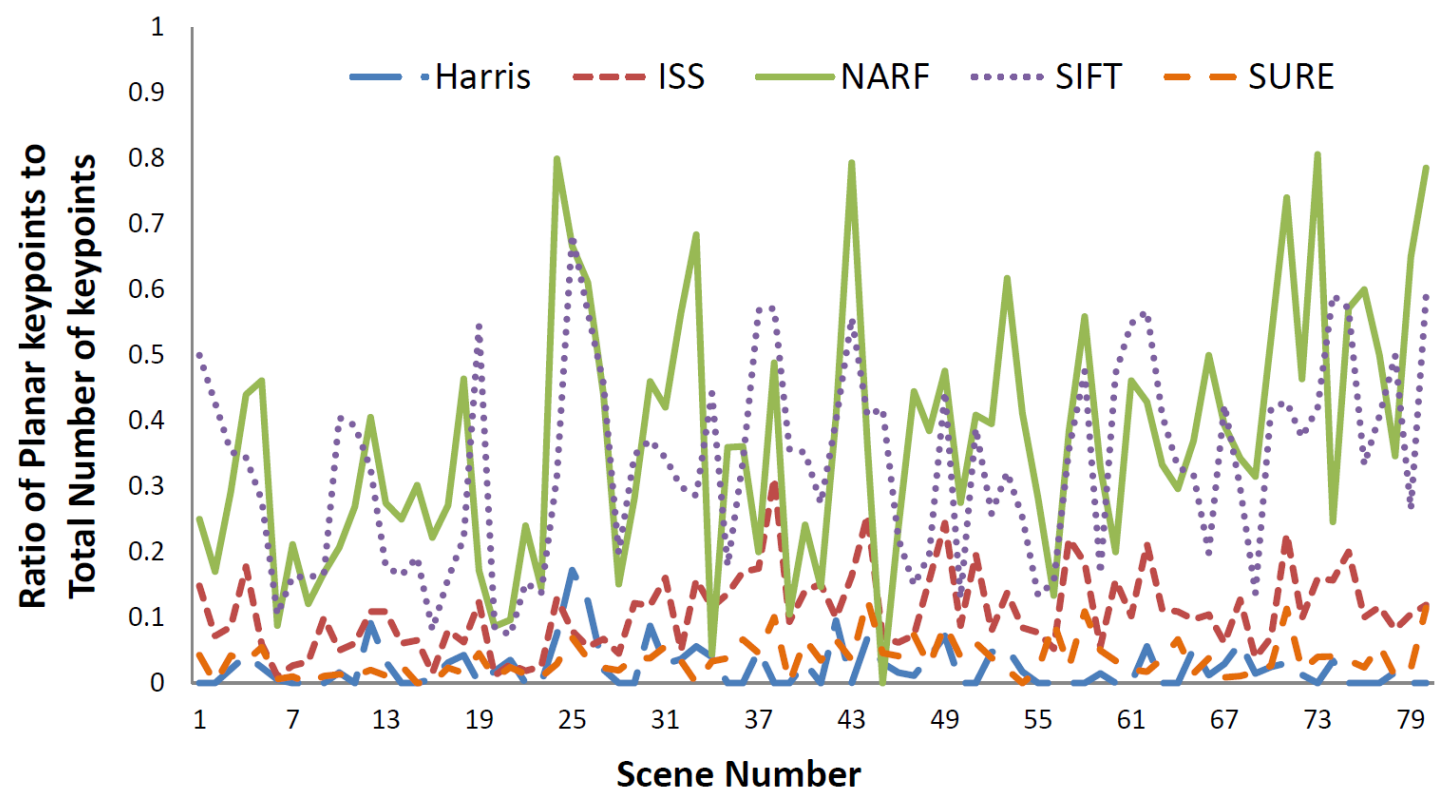

Figure 4.3: Neighbourhood Structure Test on 80 different scenes. It shows the ratio of keypoints with planar neighbourhoods to total number of keypoints that are detected by various keypoint detectors in each of the 80 scenes.

The above observations can be bolstered by the working principles behind the two keypoint detectors SURE and NARF.

\subsubsection{SURE and NARF: Working Principles}

The SURE [51] keypoint detector estimates surface normals at each point in the input point cloud. It then creates a histogram of normal orientations from the neighbourhood defined by the support radius $r$. A keypoint is extracted when the entropy of this histogram reaches a local maximum. Their algorithm measures the variation of surface normal orientations and key points are extracted where the computed surface entropy $H\left(X_{\varepsilon}\right)$ attains a local maxima. Surface entropy is calculated by $H\left(X_{\varepsilon}\right)=-\sum_{x \in X_{\varepsilon}} p(x) \log p(x)$, where $X_{\varepsilon}$ is a random variable which characterizes the distribution of surface normal orientations in a support range $\varepsilon \in R^{3}$. The SURE keypoint detector has outperformed others in the Neighbourhood Variance Test for the following reasons.

- It capitalizes on the fact that the entropy of a uniform distribution is the highest than all other distributions.

- A histogram of normal orientations in the shape of a uniform distribution resembles a keypoint neighbourhood that has diversely oriented normals. 
- Hence, in order to find the local maximum of such a histogram, the entropy based criterion provides the best results, highlighting those that show maximally and widely varying neighbourhoods, when compared to the criteria employed by other keypoint detectors.

The NARF [134] keypoint detector converts the input point cloud to a range image and finds the border pixels in it. Later, for every point other than the border points, it finds the principal curvature vector $v_{i}$ and the largest eigen value $\lambda$ of the curvature ${ }^{1}$. A weight $w$ is assigned to all points and the value of $w$ is set to 1 for the border points and $1-(1-\lambda)^{3}$ for non-border points. It considers that high values of $w$ correspond to unstable regions and defines a metric $I_{1}(p)$ for surface stability. Another metric $I_{2}(p)$ for surface variation is defined based on the largest difference between $v_{i}$ of a pair of points that lie in the support radius $r$. Based on these metrics, $I_{1}(p)$ and $I_{2}(p)$ for surface stability and variation, the NARF keypoint detector finds the keypoints that maximize their dot product.

- The surface stability metric $I_{1}(p)$ ensures that the keypoints lie on stable surfaces by monitoring the values of $w$ in the vicinity $r$ and hence moves the keypoints away from the borders. This is the reason for detecting keypoints from planar neighbourhoods, as they have stable surfaces and hence perform well in the Neighbourhood Structure Test.

- The surface variation metric $I_{2}(p)$ takes the largest difference between a pair of principal curvature vectors in the considered support radius to ensure that there is strong surface variation in the vicinity of keypoints. This is the reason for its fair performance in the Neighbourhood Variance Test.

The SURE and NARF keypoint detectors complement each other in finding keypoints from high variance and stable neighbourhoods and hence represent the scene in an effective and comprehensive manner. From now on, SURE+NARF represents the concatenation of keypoints from both the keypoint detectors, and this forms the new 3D keypoint detection module that was finally employed in SDO.

To illustrate how the SURE and NARF keypoints complement each other, a scene, as shown in Fig. 4.4, was captured and SURE, NARF and SURE+NARF keypoints were detected on it. The first row in the figure shows an RGB and depth image and the second row shows SURE keypoints (left), NARF keypoints (middle) and SURE+NARF keypoints (right) detected on the

\footnotetext{
${ }^{1}$ The principal curvature vector and the associated eigen values of the curvature are calculated by finding the largest eigen vector and eigen values of the covariance matrix of the normals that correspond to the sample points in the support radius.
} 


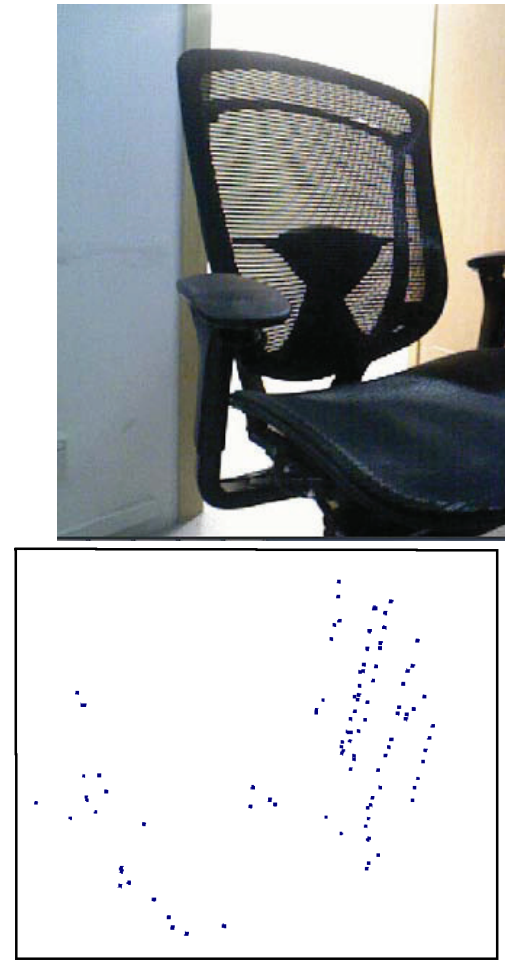

(a) SURE
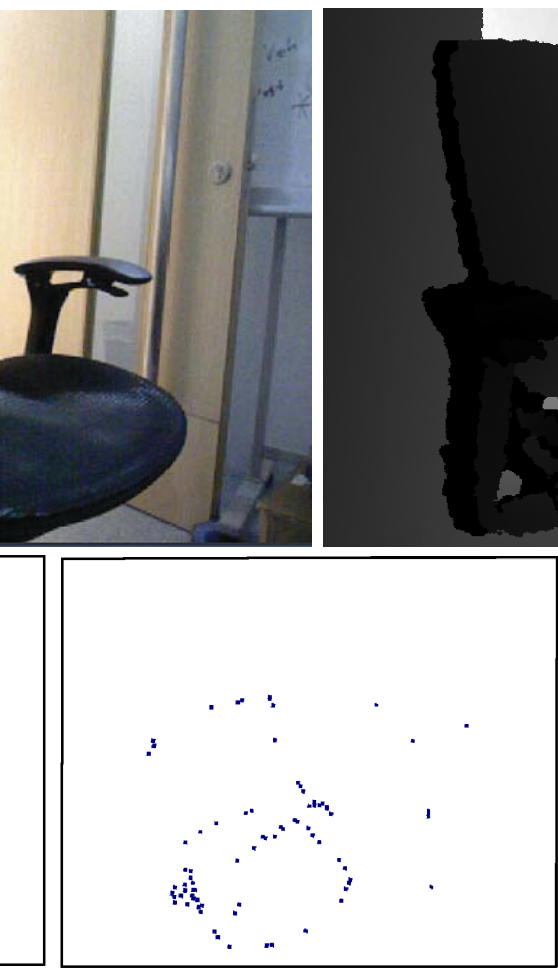

(b) NARF
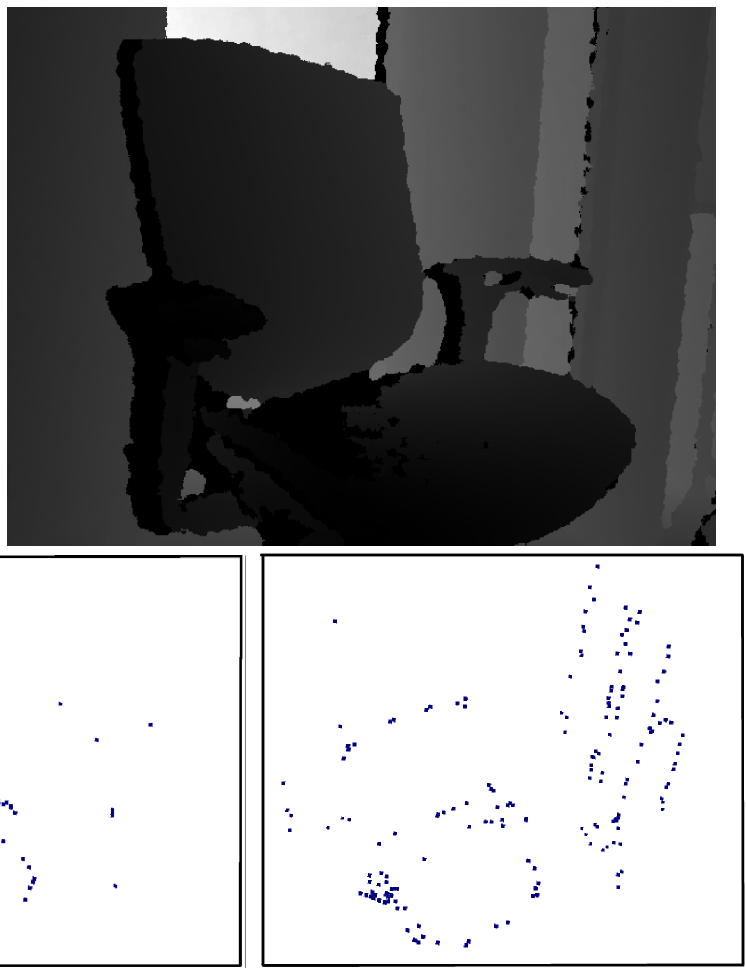

(c) SURE+NARF

Figure 4.4: The first row shows an RGB and a depth image of a scene. The second row shows (a) SURE keypoints, (b) NARF keypoints and (c) SURE+NARF keypoints extracted on the above scene. This illustrates that SURE+NARF represents the scene more comprehensively than either one of them alone.

captured scene. Most of the SURE keypoints lie on the rods and edges that are present on the door, which offers high curvature for the used support radius. NARF keypoints lie on the chair, and to be more precise, they do not lie on the borders, but lie a bit further into the center of the chair, where the surface is stable and yet has some curvature. Visually it is evident that SURE+NARF represents the scene more comprehensively than either one of them alone.

We also evaluated the performance of the considered keypoint detectors with SDO on TUM RGB-D [138] datasets, as shown in Table. 4.1. It can be seen that SURE+NARF offers better results than the others or either one of them alone.

Finally, from theoretical observations, experiments and visual analysis, it can be said that

- The SURE keypoint detector offers keypoints that come from places that have higher variance than others but the shape of their neighbourhood is mostly spherical.

- The NARF keypoint detector provides keypoints that come from both planar and spherical neighbourhoods; i.e., they represent different places that are not described by SURE. 
Table 4.1: Various Keypoint Detectors' Performance with SDO on TUM RGB-D [138] datasets. RMSE values of Absolute Trajectory Error(in metres) was used for comparison.

\begin{tabular}{ccccc}
\hline \hline Keypoint Detector & $f r 1$ xyz & frl rpy & frl desk & fr2 desk \\
\hline SURE & 0.024 & 0.138 & 0.255 & 0.143 \\
NARF & 0.031 & 0.052 & 0.506 & 0.065 \\
ISS & 0.030 & 0.135 & 0.346 & 0.234 \\
HARRIS & 0.093 & 0.467 & 0.297 & 1.379 \\
SIFT & 0.050 & 0.367 & 0.307 & 1.231 \\
SURE+NARF & $\mathbf{0 . 0 2 3}$ & $\mathbf{0 . 0 3 9}$ & $\mathbf{0 . 0 4 9}$ & $\mathbf{0 . 0 5 4}$ \\
\hline
\end{tabular}

- To represent a scene comprehensively, both of the above characteristics are required, and hence combining the SURE and NARF keypoint detectors is a better option than just choosing one of them.

We opted for comparative evaluation on debugging datasets of TUM-RGBD [138] such as frl $x y z, f r 1$ rpy, fr2 $x y z$ and fr2 rpy and visual inspection, as shown in Fig. 4.4, for different feature detectors, as the proposed evaluation criteria do not have any theoretical guarantees for highest performance. Our initial results on selecting keypoints from SURE+NARF by thresholding the variance and selecting the neighbourhood structure from which they come, did not show improvement in the performance. At times, thresholding SURE+NARF based on structure and variance has showed a slight decrease in the performance as a lesser number of SURE+NARF keypoints were passed on to the next stages. Yet, it is an interesting problem to explore a way to adaptively select 'good' keypoints from the combination of various keypoint detectors, which represent the scene comprehensively and offer good performance with SDO.

\subsection{Performance Evaluation of SDO}

\subsubsection{Computational Requirements}

For any odometry estimation algorithm, real time operation is a pre-requisite. The methods that use depth information alone $[17,103,154,121,21]$ employ a signed distance function (SDF) which demands a lot of computational power and a high memory footprint. Except SDF Tracker [21], all the other methods based on depth data alone need a GPU to cope with the computational requirements. SDO does not require a GPU and can run real time at $15 \mathrm{~Hz}$ (i.e., 15 frames per second) on a consumer laptop with an Intel core i7-2860QM CPU @2.50 GHz x 8 and 8 GB RAM. It has a very low memory footprint as it deals only with the extracted keypoints on the current frame and a model $M$ of keypoints with a fixed buffer size. 


\subsubsection{Effect of Various Modules on Operation Speed}

The real time operation of SDO at $15 \mathrm{~Hz}$ was only possible through various optimizations in different modules in the SDO pipeline. The Kinect works at $30 \mathrm{~Hz}$, i.e., in every second, 30 point clouds, each with roughly 3 million points are being given as input to SDO. Here, we deal with point clouds acquired by Kinect in indoor environments.

Voxel Grid Filtering: Firstly, the input point cloud of 3 million points is downsampled to roughly about 30,000 points and voxel grid filter also has denoising effect that inherently comes with centroid based approximation in each voxel grid. This drastic reduction in point cloud size enables SURE and NARF keypoint detectors to work at $20 \mathrm{~Hz}$. In the case where an input cloud of 3 million points is directly passed to SURE and NARF detectors, then they work at around $3 \mathrm{~Hz}$ and moreover, there was a slight reduction in the performance as noisy point clouds are directly sent to keypoint detectors. Any other denoising methods can be employed, but considering our real time requirements, voxel grid filter seemed to be the best option, where it both downsamples and filters and not just filtering alone. Moreover, voxel grid filter runs at $30 \mathrm{~Hz}$ offering no lag in the whole system pipeline.

SURE and NARF Keypoint Detectors: On the input point cloud of roughly 30,000 points from the voxel grid filter, SURE and NARF keypoint detectors, run at around $20 \mathrm{~Hz}$ and each of them detect roughly 200 keypoints each. This essentially equates to reduction of 3 million points to 400 key points considering the voxel grid filtering as a pre-processing step.

Uncertainity Modelling: This essentially converts each keypoint into a Gaussian distribution with a mean and convariance and does not add any computational lag, effectively working at $20 \mathrm{~Hz}$ as provided by the keypoint detection module.

Frame to Model Based Tracking: This is the key module that does data association and transformation estimation between model $M$ and data $D$ keypoints and constraints the overall operation speed of SDO, as it roughly works at $15 \mathrm{~Hz}$. As explained in Sec. 4.2.4, we tried various ways of data association and transformation estimation methods. In this module, it is important to hit an optimal trade-off between the computational load and the SDO's accuracy. We had to reject the possibility of employing 3D feature descriptors for matching as descriptor extraction and matching require high computational power and do not meet real time operation speeds. The other possibility was nearest neighbour based association but that was offering slightly reduced performance compared to the finally employed semi-probabilistic way of data association, which offered operation speed of $15 \mathrm{~Hz}$.

Model Update : This module does not offer any computational lag, however, the size of the Model $M$ greatly impacts the previous module and we found that employing a model $M$ 
with a size of 3000 points offered good trade-off between accuracy and speed. Increasing the model $M$ 's size to 5000 points demanded more computational power as it meant an increase in the load on frame to model based tracking module. Thus, an optimal model size of 3000 points was selected to hit a good trade-off between performance and accuracy.

Scene Complexity: Irrespective of the complexity of objects present in the input scene, voxel grid filter almost always produces roughly 30,000 points as this is mostly influenced by the Kinect point cloud's resolution. However, if the scene contains mostly planar regions, then SURE and NARF keypoint detectors work at about $25 \mathrm{~Hz}$ as they do not have much computations to perform in their non-maxima suppresion steps. However, if the input point cloud contains lot of surface variations, it runs at a lower operation speed of $18 \mathrm{~Hz}$.

\subsubsection{Accuracy and Robustness of Sparse Depth Odometry}

\subsubsection{Datasets}

There are standard, most widely used TUM RGB-D datasets provided by Sturm et al. [138] to estimate the accuracy of RGB-D camera based odometry and SLAM algorithms. Each dataset comes with an accurate ground truth trajectory captured by motion capture systems. We use these datasets to analyze SDO and compare it with the state-of-the-art. The datasets come with evaluation routines to estimate two error metrics, namely Absolute Trajectory Error (ATE) and Relative Pose Error (RPE), based on the odometry/SLAM method's trajectory and the ground truth of the dataset. It was also mentioned by Strum et al. [138] that both the error metrics are highly correlated and ATE provides results which can be visually inspected. Though ATE takes account of only translational errors, any rotational error during the motion automatically shows up as translational errors in the later frames.

\subsubsection{Results}

Fig. 4.5 shows a 2D projection of the ground truth and estimated trajectory by SDO on four datasets, namely fr 1 desk, fr3 long household and fr2 desk and fr2 desk with person. In the dataset fr 2 desk with person, a person changes the objects in the scene dynamically, whereas the other datasets have static environments and that is the reason why there is a small jitter in the estimated trajectory.

Table 4.2 provides extensive performance evaluation of SDO on the TUM RGB-D datasets. The RMSE and maximum values of the ATE and RPE error metrics are provided. The RPE values give an estimate of drift in the unit of metres/sec for translational errors and degrees/sec 

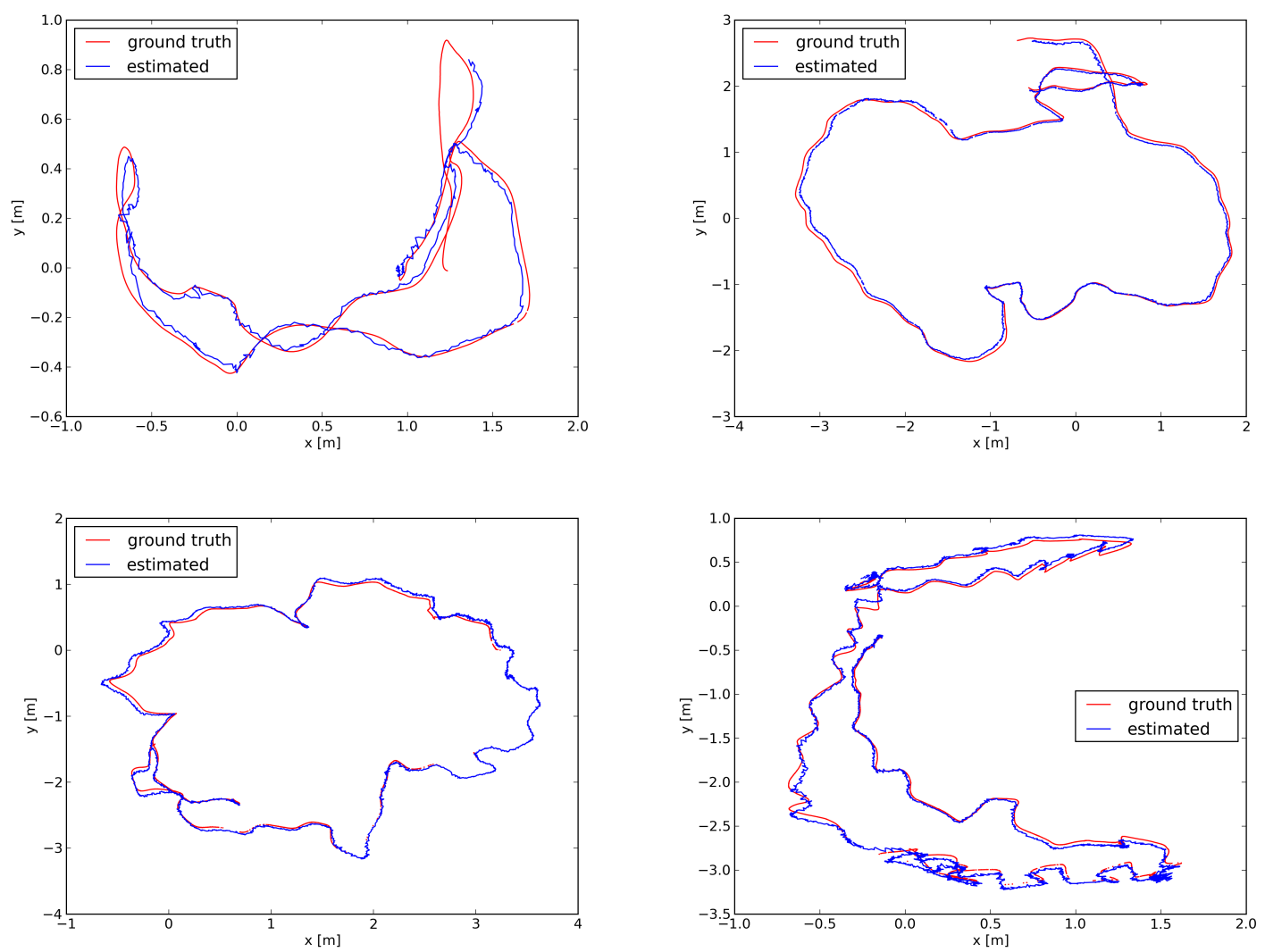

Figure 4.5: Ground truth vs. SDO's trajectory for frl desk (top left), fr3 long household (top right), fr2 desk (bottom left) and fr2 desk with person (bottom right) datasets.

in the case of rotational ones. As can be seen from Table 4.2, SDO estimates the trajectory very close to the ground truth. The average RMSE of the ATE translational error is $0.0548 \mathrm{~m}$ and RPE rotational error is $3.0782 \mathrm{deg} / \mathrm{sec}$. A point to note is that in the frl room dataset, the Kinect encounters planar surfaces without any structures in its field of view for some time; thus the error is comparatively higher than that on other datasets. To evaluate the robustness of SDO in dynamic environments, we present the performance on the datasets with dynamic objects. SDO performs well on the fr3 sitting sequences, which can be seen in Table 4.2 with datasets highlighted as dynamic. But SDO does not work on the fr3 walking sequences, where most of the foreground/scene is dynamic and only a small part of the scene/background is static.

In Table 4.3, SDO is compared with the state-of-the-art $R G B+D e p t h$ odometry methods, i.e., methods which use both RGB and depth information. Most of the published SLAM methods do not provide the error values before loop closure and graph optimization; thus their odometry accuracy is unknown. Hence to have a fair comparison, we have only used odometry methods for comparison. From the work by Whelan et al. [155], we obtain the results of the FOVIS[76], RGB-D VO[135] and FOVIS/ICP + RGB-D [155] odometry methods. As can be 
Table 4.2: Extensive Evaluation of SDO on TUM RGBD Datasets

\begin{tabular}{|c|c|c|c|c|c|c|}
\hline $\begin{array}{c}\text { Dataset } \\
\text { s-static } \\
\text { d-dynamic }\end{array}$ & $\begin{array}{l}\text { ATE } \\
\text { (in m) } \\
\text { Transl. } \\
\text { RMSE }\end{array}$ & $\begin{array}{c}\text { ATE } \\
\text { (in m) } \\
\text { Transl. } \\
\text { Max }\end{array}$ & $\begin{array}{c}\text { RPE } \\
\text { (in m/s) } \\
\text { Transl. } \\
\text { RMSE }\end{array}$ & $\begin{array}{c}\text { RPE } \\
\text { (in m/s) } \\
\text { Transl. } \\
\text { Max }\end{array}$ & $\begin{array}{c}\text { RPE } \\
\text { (deg/s) } \\
\text { Rot. } \\
\text { RMSE }\end{array}$ & $\begin{array}{c}\text { RPE } \\
\text { (deg/s) } \\
\text { Rot. } \\
\text { Max }\end{array}$ \\
\hline frl xyz (s) & 0.023 & 0.059 & 0.038 & 0.083 & 2.510 & 7.189 \\
\hline frl rpy $(\mathrm{s})$ & 0.039 & 0.170 & 0.058 & 0.200 & 7.638 & 17.350 \\
\hline frl desk (s) & 0.049 & 0.159 & 0.058 & 0.201 & 4.878 & 18.583 \\
\hline fr1 desk2 (s) & 0.071 & 0.208 & 0.073 & 0.209 & 6.715 & 14.284 \\
\hline frl room $(\mathrm{s})$ & 0.229 & 0.502 & 0.073 & 0.268 & 5.722 & 17.962 \\
\hline frl plant (s) & 0.060 & 0.131 & 0.057 & 0.157 & 4.339 & 13.554 \\
\hline fr2 $x y z$ & 0.021 & 0.110 & 0.017 & 0.114 & 0.839 & 3.943 \\
\hline fr2 rpy (s) & 0.021 & 0.070 & 0.017 & 0.077 & 1.022 & 3.950 \\
\hline fr2 desk (s) & 0.054 & 0.120 & 0.028 & 0.097 & 1.601 & 4.738 \\
\hline fr3 long household (s) & 0.057 & 0.136 & 0.026 & 0.084 & 1.553 & 5.174 \\
\hline fr2 desk with person (d) & 0.047 & 0.133 & 0.031 & 0.177 & 1.515 & 7.331 \\
\hline fr3 sitting static $(\mathrm{d})$ & 0.018 & 0.060 & 0.025 & 0.069 & 0.984 & 3.136 \\
\hline fr3 sitting $x y z(\mathrm{~d})$ & 0.044 & 0.128 & 0.039 & 0.152 & 1.661 & 4.755 \\
\hline fr3 sitting rpy $(\mathrm{d})$ & 0.041 & 0.170 & 0.053 & 0.237 & 4.041 & 17.242 \\
\hline fr3 sitting halfsphere (d) & 0.049 & 0.156 & 0.053 & 0.184 & 4.156 & 17.193 \\
\hline Avg. Error & 0.0548 & 0.1541 & 0.0430 & 0.1539 & 3.0782 & 10.4256 \\
\hline
\end{tabular}

Table 4.3: Comparision of Median and Maximum values of Absolute Trajectory Error (in metres) with $R G B+$ Depth odometry algorithms

\begin{tabular}{|c|c|c|c|c|c|c|}
\hline \multirow{2}{*}{ Algorithm } & \multicolumn{2}{|c|}{ frl desk } & \multicolumn{2}{c|}{ fr2 desk } & \multicolumn{2}{c|}{ frl room } \\
& Median & Max & Median & Max & Median & Max \\
\hline SDO & $\mathbf{0 . 0 4 1}$ & 0.159 & $\mathbf{0 . 0 4 9}$ & $\mathbf{0 . 1 2 0}$ & 0.171 & 0.502 \\
\hline FOVIS [76] & 0.221 & 0.799 & 0.112 & 0.217 & 0.238 & 0.508 \\
\hline RGB-D VO [135] & 0.094 & $\mathbf{0 . 1 3 8}$ & 0.132 & 0.372 & 0.447 & 0.872 \\
\hline FOVIS/ICP + RGB-D [155] & 0.068 & 0.231 & 0.118 & 0.346 & $\mathbf{0 . 1 5 2}$ & $\mathbf{0 . 4 1 9}$ \\
\hline
\end{tabular}

seen from Table 4.3, SDO is more accurate than the state-of-the-art on the frl desk and fr2 desk datasets. However on the frl room dataset, it does not give the best performance because in this dataset, the Kinect encounters planar surface where there is no structure information.

In Table 4.4, SDO is compared to the state-of-the-art Depth alone methods [103, 17, 21]. The main difference between these methods and SDO is that all of them are dense methods, i.e., they use all of the depth data for odometry estimation, and SDO is a sparse method which only uses a few key points for odometry estimation. All these dense methods employ SDF (signed distance function) and have high memory footprint. Except SDF Tracker [21], the 
Table 4.4: Comparison of RMSE values of Absolute Trajectory Error( in metres) on TUM RGB-D datasets with Depth alone methods

\begin{tabular}{ccccc}
\hline \hline Dataset & $\begin{array}{c}\text { SDO } \\
\text { no GPU }\end{array}$ & $\begin{array}{c}\text { KinFu LS } \\
{[103]} \\
\text { (Res. 256) } \\
\text { needs GPU }\end{array}$ & $\begin{array}{c}\text { SDF method } \\
{[17]} \\
\text { (Res. 256) } \\
\text { needs GPU }\end{array}$ & $\begin{array}{c}\text { SDF Tracker [21] } \\
\text { no GPU }\end{array}$ \\
\hline frl xyz & 0.023 & 0.026 & 0.021 & $\mathbf{0 . 0 1 4}$ \\
frl rpy & $\mathbf{0 . 0 3 9}$ & 0.133 & 0.047 & 0.136 \\
frl desk & 0.049 & 0.057 & 0.038 & $\mathbf{0 . 0 3 3}$ \\
fr1 desk2 & 0.071 & 0.420 & $\mathbf{0 . 0 6 1}$ & 0.230 \\
frl room & 0.229 & failed & $\mathbf{0 . 1 2 1}$ & 1.111 \\
fr1 plant & 0.060 & 0.598 & $\mathbf{0 . 0 4 7}$ & 0.756 \\
fr2 xyz & $\mathbf{0 . 0 2 1}$ & - & $\mathbf{0 . 0 2 1}$ & 0.122 \\
fr2 rpy & $\mathbf{0 . 0 2 1}$ & - & 0.024 & 0.110 \\
fr2 desk & $\mathbf{0 . 0 5 4}$ & - & - & 0.662 \\
fr3 long household & 0.057 & 0.064 & $\mathbf{0 . 0 3 9}$ & 0.960 \\
\hline Avg. Error & 0.0624 & 0.216 & 0.0465 & 0.4134 \\
\hline
\end{tabular}

other two methods rely on a GPU to meet their computational requirements. The results of KinFu LS [103] and the SDF method [17] are taken from Bylow et al. [17]. The results of SDF Tracker [21] were generated by running their open source implementation on the datasets. SDO outperforms the state-of-the-art on $f r 1$ rpy, fr2 $x y z, f r 2$ rpy and $f r 2$ desk datasets. It can be seen from Table 4.4 that SDO's performance is comparable to the state-of-the-art, with deviation being less than $0.02 \mathrm{~m}$, as shown by the average error taken over the datasets for which the results are available. SDO can also work on the fr2 desk dataset for which SDF method [17] can not be evaluated.

\subsubsection{D Mapping with SDO}

A stair-case can be encountered in indoor environments and the wheel odometry from mobile robots is not reliable. Thus to show the effectiveness of depth odometry, we create a map of indoor stair-case in Fig. 4.6 (a). Figure 4.7 shows multiple views of the octomap with 0.01 metre resolution created by depth odometry on fr3 long household dataset. A coloured mesh presentation of the same dataset created with the help of volumetric mapping [136] is shown in in Fig. 4.6(b). The coloured mesh is shown to create a visually appealing representation, but we do not use colour for pose estimation. 


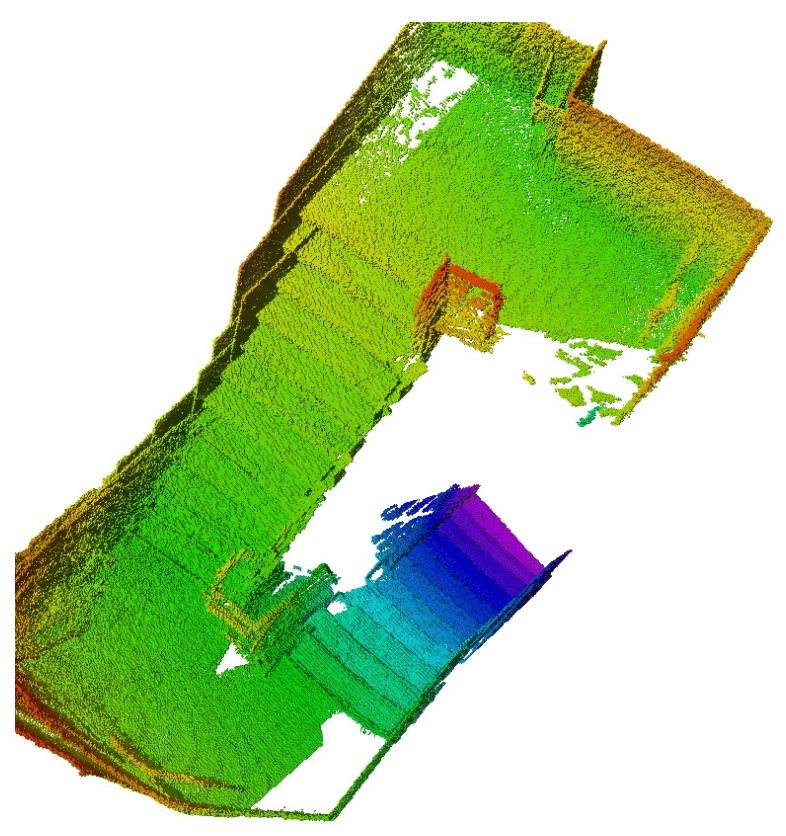

(a) Stair-case

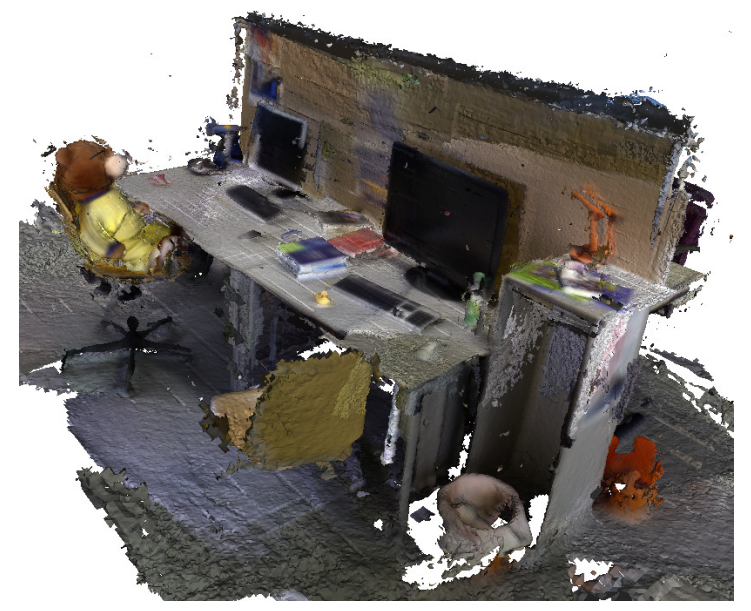

(b) 3D reconstruction of $f r 3$ long household dataset

Figure 4.6: An octomap of a stair-case and a mesh created using volumetric mapping [136] with SDO are shown in this figure.

\subsection{Conclusion}

In this chapter, we presented Sparse Depth Odometry (SDO), an incremental pose estimation method that works by extracting 3D keypoints on depth data from Kinect-like sensors. The reason behind accurate pose estimation and real time execution without a GPU, is the proposed 3D keypoint detection module. The proposed keypoint detection module comprises of two keypoint detectors, SURE and NARF. An extensive neighbourhood structure and variance analysis was performed on various existing keypoint detectors before choosing SURE and NARF. It was found that SURE keypoints came from highly varying regions while NARF keypoints came from both curved, slightly planar and stable regions that SURE can not represent. These both keypoint detectors complemented each other and effectively represented the scene in a more comprehensive manner, that works well with nearest neighbour association as employed for real time operation in SDO. Finally, SDO powered with the proposed keypoint detection module, has the qualities of running online without a GPU, a smaller memory footprint and is structurally different (as it does not use a signed distance function) when compared to other Depth-alone odometry methods. SDO can also be used to augment the existing RGB-D methods that fail to work in places with no illumination.

This concludes the 3D keypoint detection part of this thesis, which dealt with the proposal 

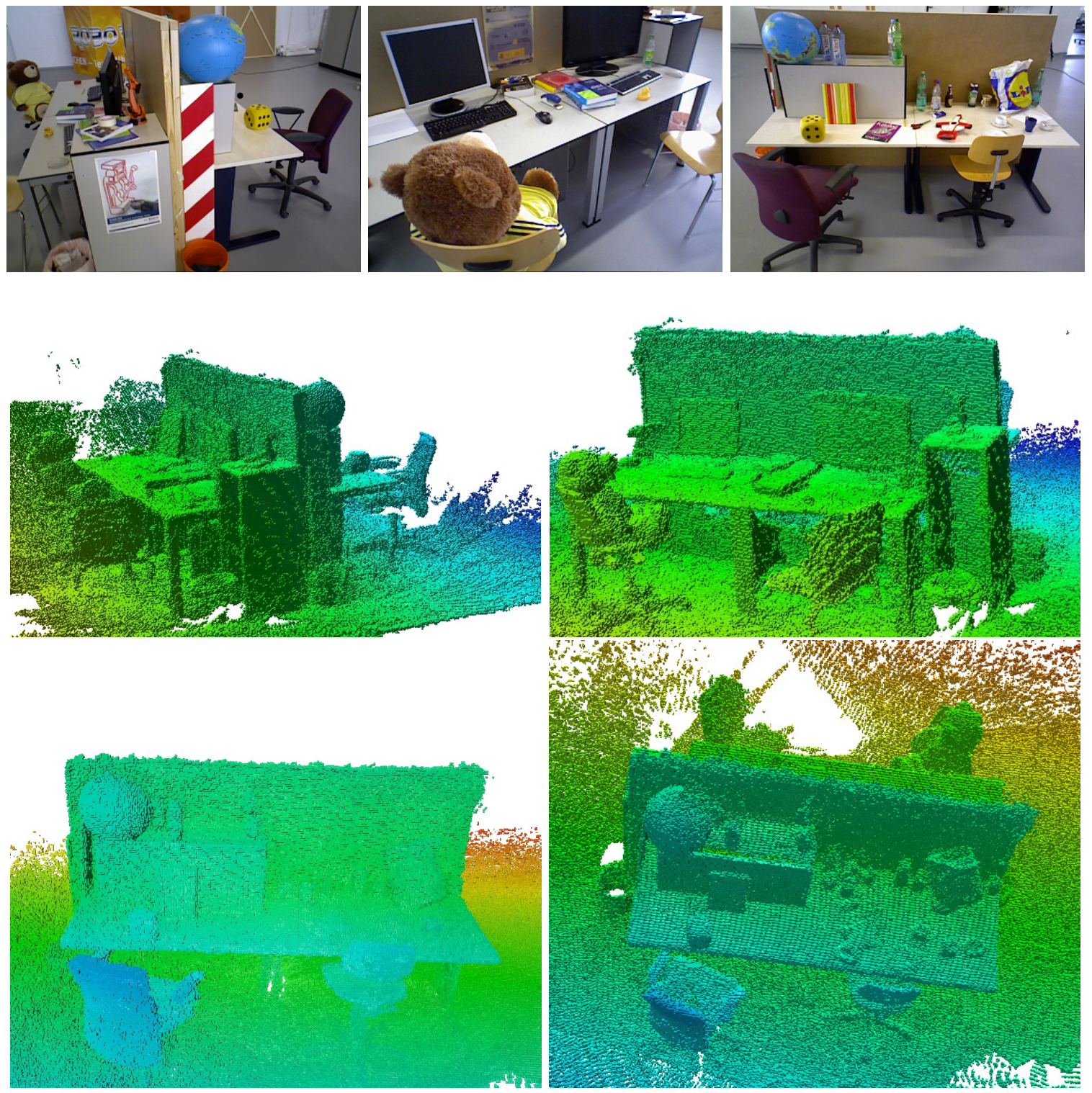

Figure 4.7: Multiple views of octomap with $0.01 \mathrm{~m}$ resolution created using depth odometry on fr3 long household dataset. Three RGB images from the fr3 long household dataset are shown to illustrate how the environment looks.

of a generic 3D keypoint detector and a specific keypoint detection module for a 3D perception application. In the next part of the thesis, we present our works on $3 \mathrm{D}$ feature description. 


\section{Chapter 5}

\section{DHoPD: A Fast Low Dimensional Real-valued 3D Feature Descriptor}

\subsection{Introduction}

Starting from this chapter, we focus on 3D feature description and present new proposals that are computationally and memory efficient. Feature descriptors essentially encode the characteristics of point distributions around the keypoints into a multidimensional vector. In most of the 3D perception applications such as 3D object modelling [65], 3D object recognition [7, 63], partial 3D object retrieval [129, 47] and point cloud registration [126, 112], 3D feature descriptor based keypoint matching plays a crucial role by determining the accuracy, memory and computational requirements. And with mobile devices equipped with depth sensors such as Lenovo Phab 2 Pro smartphone and Google Tango, there is a need to develop applications that are computationally efficient and have low memory footprint. Therefore, reducing both the memory required for 3D feature descriptors and their computational needs will greatly impact the overall memory and computational footprints of the target application.

\section{Relevant state-of-the-art 3D descriptors employed for comparative evaluation}

Existing 3D feature descriptors were reviewed in Chapter. 2, and here we briefly present those state-of-the-art 3D descriptors that we employ for comparative evaluation, which comprise of SHOT [128], FPFH [124], 3DSC [57], USC [143] descritpors. Signature of Histograms of normal OrienTations (SHOT) descriptor tries to mimic SIFT [90] descriptor from 2D image domain and hence, employs a histogram of gradients (surface normals in 3D domain) to represent the point distributions in each partition of the superimposed 3D spherical grid. FPFH descriptor is proposed as an improved version of PFH [125], which is based on Darbaux frame calculated between the keypoint and its neighbours. In FPFH, firstly Simplified PFH's (SPFH) 
are computed between the keypoint and each of its neighbours and then the descriptor is constructed as a weighted sum of SPFH's. 3D Shape Contexts (3DSC) [57] superimpose a spherical grid aligned with the surface normal at the considered keypoint and calculate a weighted sum of points that fall in each partition along radial, azimuth and elevation directions. Unique Shape Contexts (USC) [143] was proposed as an extension to 3DSC, wherein a unique and repeatable local reference frame is employed to superimpose the $3 \mathrm{D}$ spherical grid rather than a surface normal. Lastly, RoPS [66] descriptor is constructed by accumulating various statistics calculated from the distribution matrices that are constructed by rotating the 3D surface patch along $x, y, z$ axes and projecting it onto $y z, z x, x y$ planes. However, we do not compare with RoPS descriptor, because RoPS requires mesh connectivity information and hence it can not readily work on raw point clouds that have $[x, y, z]$ information alone. Mesh computation from raw point clouds involves calculation of surface normals and other post-processing steps that add significant computational overhead. The proposed low dimensional 3D descriptor and all other above mentioned descriptors in this chapter work directly on raw point clouds, which are readily available from depth sensors and do not require the computation of mesh representation.

\section{Drawbacks of the existing 3D descriptors: Memory or Computational Complexity}

While the memory footprint and matching complexity reciprocate with descriptor's dimensionality, the extraction complexity depends on the hand crafted design of the descriptor. Though FPFH has only 33 dimensions, its extraction time is significantly higher compared to others because the effective support size for descriptor extraction becomes twice for computing SPFH [124]. With this, as the support size increases, the number of points in $3 \mathrm{D}$ volume increase exponentially, hence making FPFH computationally expensive in high density point clouds or with larger support size [64]. On the other hand, USC and 3DSC, with a dimensionality of 1980, employ an excessively high number of bins and represent each bin, only with a single value, hence they lose on performance and have high computational complexity. In the case of SHOT descriptor with 352 dimensions, surface normal computation takes up most part of the computational power, however, their idea of imitating SIFT by representing each grid with a histogram (not with a single value as employed in 3DSC and USC) of normals pays off with better performance on point clouds that have small surface variations.

SHOT (352 dim) and USC (1980 dim) are relatively faster in descriptor extraction compared to FPFH but demand higher memory footprint and matching complexity because of their dimensionality. Even if PCA based dimensionality reduction is employed, it would still be necessary to extract those high dimensional descriptors in the firstplace on the target mobile 
devices and store the PCA transformation matrices, thus resulting in high memory and computational footprints.

Contribution: Therefore, in this chapter, we propose 3DHoPD, a low dimensional (18-dim) $3 \mathrm{D}$ descriptor that is extremely fast to compute and offers stable and competitive performance and a tailored descriptor matching technique. The novelty lies in the design of 3DHoPD (3D+HoPD) involving two steps:

1. 3D: We propose to transform the detected 3D keypoints to a new 3D space where keypoints arising from similar 3D surfaces lie close to each other.

In this new 3D space, for a given source keypoint, a list of probable keypoint matches with high recall can be retrieved with a simple radial search. This reduces the search space by $90 \%$ by removing the false positive matches and aids in descriptor matching.

2. HoPD: From this set of high probable keypoint matches, the exact match is then found by proposing a fast 3D descriptor, HoPD (Histogram of Point Distributions).

The source code of the proposed 3DHoPD descriptor will be made available at https://sites.google.com/site/3dhopd/

\subsection{DHoPD: The Proposed 3D Feature Descriptor}

Lets consider an input source point cloud $P_{\text {source }}$ on which $N$ keypoints, $K_{n}$, where $n=$ $\{1,2, \ldots, N\}$ are detected using a 3D keypoint detector. Then, for each of these source keypoints, $K_{n}$, a 3DHoPD feature descriptor is created from its neighbourhood surface Sur face $_{n m}$ containing $M$ points, where $m=\{1,2, \ldots, M\}$. The neighbourhood surface or the 3D surface patch around the keypoint is determined by the support size used to construct the $3 \mathrm{D}$ descriptor. Notation example: A keypoint $K_{i}$ has a Surface $_{i m}$, where $i$ represents the keypoint index and $m$ represents the points in the $3 \mathrm{D}$ surface patch around the keypoint $K_{i}$.

\subsubsection{Construction}

The 18 dimensional 3DHoPD descriptor encodes the 3D keypoint position in the first three dimensions and the HoPD descriptor in the next 15 dimensions. 


\subsubsection{3D Keypoint Transformation technique to encode the 3D keypoint position}

The considered 3D keypoint is transformed to a new 3D space and its new 3D coordinates are stored in the first three dimensions of the 3DHoPD descriptor. The task of extracting feature descriptors around keypoints and matching them to find correspondences can be seen as trying to find exact 3D keypoint correspondences, where each keypoint correspondence arises from similar 3D surface patch/neighbourhood. Hence, we transform the keypoints to a new 3D space where the keypoints arising from similar 3D surfaces lie close to each other. To achieve this, let us consider a 3D surface patch Sur face $_{i m}$ with $m$ neighbourhood points around the keypoint $K_{i}$. First, we find the mean point mean $n_{p t}$ i.e., the centroid of all other points in the considered 3D surface patch Surface $_{i m}$. Second, we subtract the found mean $n_{p t}$ from all the 3D points

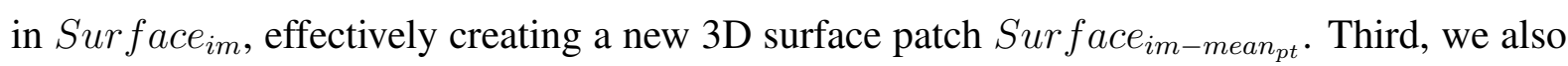
subtract the found mean $_{p t}$ from the considered keypoint $K_{i}$ as well, resulting in $K_{i-m e a n}$. Finally, we estimate the local reference frame $[\mathbf{R F}]_{3 \times 3}$, as explained later, from this new $3 \mathrm{D}$ surface patch Surface im-mean $_{p t}$, and then transform the keypoint $K_{i-\text { mean }_{p t}}$ to a new 3D space as shown below:

$$
\left[K_{i R F}\right]_{3 \times 1}=[\mathbf{R F}]_{3 \times 3}\left[K_{i-\text { mean }_{p t}}\right]_{3 \times 1}
$$

where $K_{i R F}$ represents the new 3D coordinates (in new 3D space) of the considered keypoint $K_{i}$ arising from a 3D surface patch Surface $_{i m}$. And, $\left[K_{i R F}\right]_{3 \times 1}$ forms the first three dimensions of the constructed 3DHoPD descriptor.

Local Reference Frame: It is estimated from the eigenvectors of the modified covariance matrix $\mathbf{C}$ calculated from the points in the 3D surface patch Surface $_{i m-\text { mean }_{p}}$, as shown in Eqn. 5.2. Similar to the local reference frame calculated in SHOT [128], the 3D points, Surface $_{\text {im-mean }_{p t}}$, where $m=\{1,2, \ldots, M\}$, that lie in the neighbourhood defined by support radius $\mathbf{r}$, are weighed based on their distance from the considered keypoint $K_{i-\text { mean }_{p t}}$ as shown in Eqn. 5.2. For readability, we represent $M$ points in 3D surface $S u r f a c e_{i m-\text { mean }_{p t}}$ by $\mathbf{q}_{\mathbf{m}}$ and $K_{i-\text { mean }_{p t}}$ by q.

$$
\mathbf{C}=\frac{1}{\sum_{m: d_{m} \leq \mathbf{r}}\left(\mathbf{r}-d_{m}\right)} \sum_{i: d_{m} \leq \mathbf{r}}\left(\mathbf{r}-d_{m}\right)\left(\mathbf{q}_{\mathbf{m}}-\mathbf{q}\right)\left(\mathbf{q}_{\mathbf{m}}-\mathbf{q}\right)^{\mathbf{T}}
$$

where $d_{m}=\left\|\mathbf{q}_{\mathbf{m}}-\mathbf{q}\right\|_{2}$. To create a unique local reference frame and remove the sign ambiguity, the direction of the local $\mathrm{x}$ and $\mathrm{z}$ axes are oriented towards the majority direction of the vectors that they represent [15]. Finally, the local $y$ axis is obtained by the cross product of $\mathbf{z}$ and $\mathbf{x}$, i.e., $\mathbf{y}=\mathbf{z} \times \mathbf{x}$. The local reference frame is defined as $[\mathbf{R F}]_{3 \times 3}=\left[\mathbf{x}^{T} \mathbf{y}^{T} \mathbf{z}^{T}\right]^{T}$. 


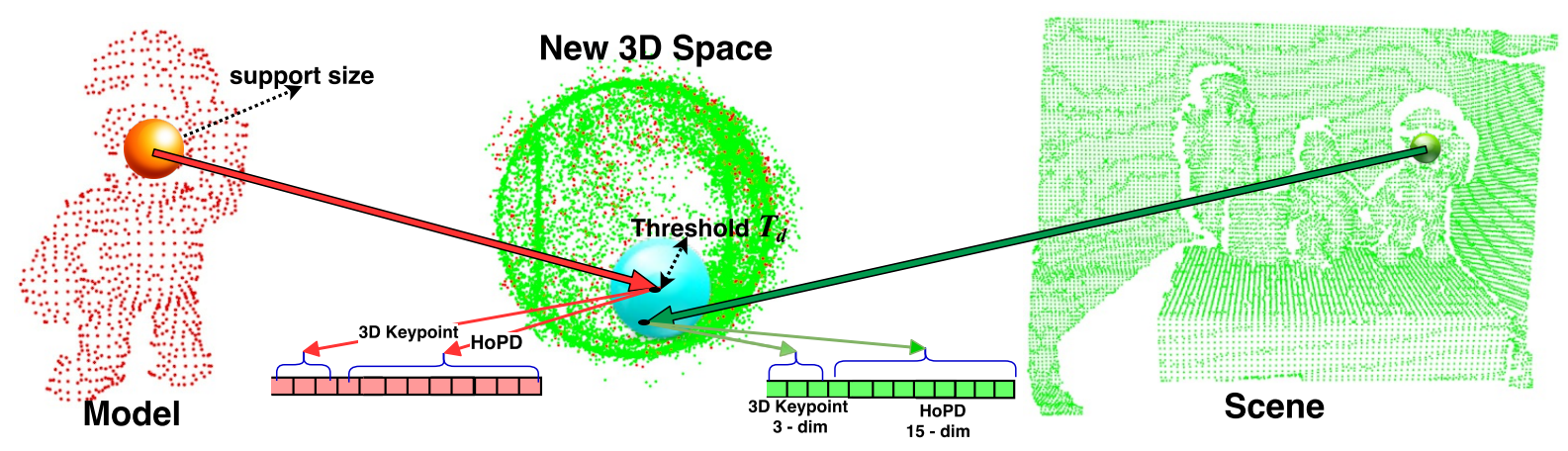

Figure 5.1: The uniform model keypoints are shown in red while the scene keypoints are shown in green. The red and green spheres represent the support size for LRF and descriptor construction. There is one to one correspondence between keypoints in original space and the new 3D space. The model and scene keypoints are transformed to a new 3D space with the local reference frame. Then, for every model keypoint, a list of scene keypoints are retrieved with a radial search of radius $T_{d}$ (shown as cyan colored sphere in new 3D space). From this retrieved list of scene keypoints, the one whose HoPD descriptor is closest to the considered model keypoint's descriptor, is assigned as the match.

Rotational Invariance : To achieve rotational invariance, the standard procedure that is followed in the most widely used SIFT [90] descriptor and some of the best 3D feature descriptors such as SHOT and RoPS descriptors, is applied in the proposed 3DHoPD descriptor as well. These state-of-the-art 2D and 3D descriptors find a principal direction of the patch/neighbourhood around a keypoint and rotate/transform the patch based on the estimated local reference frame. The rotational invariance in 3DHoPD is achieved by transforming the 3D surface patch around the considered 3D keypoint using the estimated local reference frame $[\mathbf{R F}]_{3 \times 3}$, as described above.

Intuition behind the 3D Keypoint Transformation technique: In the first step of encoding the 3D keypoint position, we transform the 3D keypoints arising from similar 3D surface patches into a new space where they lie in vicinity of each other. To achieve this, we remove the mean point (geometric offset) or the centroid $\left(\right.$ mean $\left._{p t}\right)$ of local surface patch from all its points and then transform the keypoint to a new 3D space using the local reference frame. This local reference frame $[\mathbf{R F}]_{3 \times 3}$ estimated from the covariance matrix approximately captures the local 3D surface pattern. It cannot be said that the closest point in the new 3D space corresponds to the true feature match because of various practical abnormalities, such as keypoint detection ambiguity [147], noisy sensor data and the inability of the local reference frame to accurately capture the variations in the local surface. However, the true match lies in its vicinity, hence drastically reducing the search space and resulting in a small set of points to find the exact keypoint match from. 
In Fig. 5.1, we show uniformly extracted model keypoints in red to the left, and uniform scene keypoints in green to the right. The red and green spheres on model and scene represent the support size used to construct the local reference frame and the HoPD descriptors. These model and scene keypoints are then transformed into a new 3D space that spans the volume of a unit 3D sphere, by removing the offset and multiplying them with a normalized local reference frame. There is a one to one correspondence between every model keypoint in their original space and the new 3D space, and similarly between every scene keypoint in their original space and the new $3 \mathrm{D}$ space.

Now, to find a match for the considered model keypoint in this new 3D space, we perform a radial search with a radius of $T_{d}$ as shown by a cyan colored sphere and retrieve a list of probable matches. From this list of probable matches, the one whose HoPD descriptor is closest to the considered model keypoint's HoPD descriptor, is considered as a match. This proposed 3D keypoint transformation method drastically reduces the search space, removes about $90 \%$ of false positives as shown later, and makes the job of descriptors easier, thus enabling the design of fast descriptors that offer good performance.

\subsubsection{Histogram of Point Distributions (HoPD) Descriptor}

The HoPD descriptor encapsulates the actual point distributions in the neighbourhood of the considered 3D keypoint into histogram. Following the same notation as in Sec. 5.2.1, the 3D surface patch around a keypoint $K_{i}$ from the source point cloud would be Sur face $_{i m-\text { mean }_{p t}}$ with $m$ neighbourhood points in the support region and mean $n_{p t}$ being the centroid. This $3 \mathrm{D}$ surface patch is transformed with the estimated local reference frame $[\mathbf{R F}]_{3 \times 3}$ to achieve rotational invariance. To construct HoPD, a $D$-dimensional histogram is created for $x$ axis, by finding the minimum and the maximum $x$ coordinate value of the points in the 3D surface patch. Then the found range between the minimum and the maximum is divided into $D$-bins and a $D$ dimensional histogram is constructed by counting the number of points that fall into each bin. The same procedure is repeated for $y$ and $z$ axes too, effectively creating a $3 \times D$-dimensional HoPD descriptor. Our experiments have showed that $D=5$ offers a good trade-off between performance and dimensionality, hence resulting in a 15-dimensional HoPD descriptor. The advantage of the HoPD descriptor is that its extremely fast to compute, as it does not require any computation of surface normals.

The idea is to first generate a list of highly probable matches based on the '3D' keypoint position encoded in the first three dimensions and then find an exact match with the help of fast 15-dim 'HoPD' descriptor. 


\subsubsection{Matching}

Capitalizing on the fact that the 3D keypoints arising from similar 3D patches lie close to each other in the new 3D space, we perform the matching of 3DHoPD descriptors as follows. Firstly, the keypoints extracted on the source and the target point clouds are transformed in to the new 3D space. Then, for every source 3D keypoint $K_{i R F \text { source }}$ in the new $3 \mathrm{D}$ space, we find a list of nearest target 3D keypoints $K_{\text {iRFtarget }}$ in the new 3D space that lie within a distance threshold $T_{d}$. We perform a radial nearest neighbour search with radius $T_{d}$ by employing KdTree for fast retrieval. Hence, we use the first three dimensions of 3DHoPD descriptor to generate the list of probable matches for every source keypoint. Then, in the next step, we find the exact match for the considered source keypoint from the above generated list of probable matches by employing Euclidean metric to find the exact nearest neighbour in the 15-dim HoPD descriptor's space.

\subsection{Experimental Evaluation}

We perform extensive evaluation on four publicly available datasets [64] namely, Retrieval, Queens, Random Views and Kinect, based on widely used precision-recall curves [97, 64, 128, 66] for descriptor matching experiments. These datasets come with models, scenes and the groundtruth 3D transformation between them. The term 'model' represents an object point cloud, while the term 'scene' represents a point cloud containing various objects in different orientations, noise, occlusion and clutter.

\subsubsection{Evaluation of the 3D Keypoint Transformation Technique}

The main contribution of this work is to transform 3D keypoints to a new 3D space, based on the local reference frame, so that the keypoints arising from similar 3D surface patches lie close to each other. We perform the following experiments to evaluate and validate the proposed concept on all four datasets, whose point cloud data comes arises from various sensing modalities. Each dataset comes with a set of scene-model pairs and a groundtruth 3D transformation between them.

1. ISS [162] keypoints with parameters as mentioned later, are detected on a scene and they are transformed on to the model based on the available groundtruth transformation, hence the groundtruth 3D keypoint correspondences are known.

2. The model keypoints and the scene keypoints are transformed into the new 3D space by multiplying with the estimated LRF as mentioned in Sec. 5.2.1. 
Table 5.1: This table presents average number of (i) model keypoints, (ii) scene keypoints, (iii) the average size of the list of probable scene keypoint matches retrieved for every model keypoint (based on the '3D' keypoint transformation in the new 3D space) and (iv) the percentage of the retrieved lists that actually contain the groundtruth keypoint match, in each of the datasets.

\begin{tabular}{|c|c|c|c|c|}
\hline Dataset & $\begin{array}{l}\text { Model Keypoints } \\
\text { Avg. }\end{array}$ & Scene Keypoints & $\begin{array}{c}\text { Avg. size of } \\
\text { the retrieved list } \\
(\% \text { of Scene Keypoints retrieved })\end{array}$ & $\begin{array}{l}\text { \% of retrieved lists } \\
\text { with groundtruth } \\
\text { (Recall) }\end{array}$ \\
\hline \multicolumn{5}{|c|}{ Threshold: $T_{d}=0.005$} \\
\hline Retrieval & 63 & 63 & $5->(7.9 \%$ of scene keypoints $)$ & $99.28 \%$ \\
\hline Random Views & 33 & 99 & $4->(4.04 \%$ of scene keypoints $)$ & $22.5 \%$ \\
\hline Queens & 175 & 862 & $28->(3.24 \%$ of scene keypoints $)$ & $14.7 \%$ \\
\hline Kinect & 88 & 1254 & $48->(3.82 \%$ of scene keypoints $)$ & $35.61 \%$ \\
\hline \multicolumn{5}{|c|}{ Threshold: $T_{d}=0.0075$} \\
\hline Retrieval & 63 & 63 & $9->(14.28 \%$ of scene keypoints $)$ & $99.61 \%$ \\
\hline Random Views & 33 & 99 & $10->(10.10 \%$ of scene keypoints $)$ & $34.08 \%$ \\
\hline Queens & 175 & 862 & $81->(9.39 \%$ of scene keypoints $)$ & $28.01 \%$ \\
\hline Kinect & 88 & 1254 & $113->(9.01 \%$ of scene keypoints $)$ & $50.40 \%$ \\
\hline \multicolumn{5}{|c|}{ Threshold: $T_{d}=0.02$} \\
\hline Retrieval & 63 & 63 & $31->(49.2 \%$ of scene keypoints $)$ & $99.76 \%$ \\
\hline Random Views & 33 & 99 & $50->(50.5 \%$ of scene keypoints $)$ & $77.8 \%$ \\
\hline Queens & 175 & 862 & $559->(64.84 \%$ of scene keypoints $)$ & $78.8 \%$ \\
\hline Kinect & 88 & 1254 & $611->(48.72 \%$ of scene keypoints $)$ & $84.28 \%$ \\
\hline
\end{tabular}

3. In this new 3D space, for every model keypoint, a radial search is performed with a radius of $T_{d}$ and a list of nearest neighbours, which form the set of probable scene keypoint correspondences, are retrieved.

4. Finally, we check, if the list of retrieved probable scene keypoint correspondences for every model keypoint contains the actual groundtruth scene keypoint match or not.

The results are presented in Table 5.1 and for each dataset. The table shows the average number of (i) model keypoints, (ii) scene keypoints, (iii) average size of the retrieved list of scene keypoints in the new 3D space for every model keypoint, and finally (iv) the percentage of retrieved lists that contain the groundtruth correspondence in them. In the fourth column of Table 5.1, we also present the size of the retrieved list in terms of the percentage of scene keypoints, so that it gives an idea of how many false positives are removed. We run the experiments with three different radial thresholds, $T_{d}=\{0.005,0.0075,0.02\}$ that determines the number of the nearest neighbours retrieved in the new 3D space. In all the experiments, the support size of descriptors is set to $0.06 \mathrm{~m}$, which is the same for local reference frame computation as well. In the Retrieval dataset, the models and the scenes are essentially the same, which is the reason for having same number of model and scene keypoints in Table 5.1. However, scenes come with added Gaussian noise of $\sigma=0.1 \mathrm{mr}$ (mesh resolution) and scene-model pairs are highly oriented/rotated in this dataset. 
It can be seen from Table 5.1 that, for each dataset, as $T_{d}$ increases, the 'avg. size of the retrieved list' and the '\% of retrieved lists with groundtruth (recall)' also increases. As the size of the retrieved list increases, many false positives creep in and a highly descriptive descriptor is required to filter the false matches and find the exact true keypoint match. If the retrieved list is small, which is achievable with small $T_{d}$, the $\%$ of retrieved lists with groundtruth also decrease. Hence, it is ideal to choose a reasonable value that gives a fair size of retrieved list and in all the descriptor matching and evaluation experiments carried out later on, we employed $T_{d}=0.0075$, as it offered good trade-off between the size of the retrieved list and the $\%$ of groundtruth matches it contains.

For 3D keypoint matching, each model keypoint descriptor has to be matched/compared against each and every scene keypoint descriptor to find the final exact match. The biggest advantage with the proposed 3D keypoint transformation is that, by using just three dimensions of the descriptor which is the 3D keypoint location, a small list of highly probable source keypoint matches can be retrieved and hence, a simple and fast descriptor can be designed to find the exact match. It can be seen from Table 5.1 that, by employing a threshold $T_{d}=0.0075$, approximately only $10 \%$ of the source keypoints need to be searched to find an exact match for every model keypoint and effectively reduces the search space by $90 \%$. This proposed '3D' keypoint transformation to a new 3D space greatly removes the false positives and makes the job of descriptors easier. Our experiments have shown the practical effectiveness of setting the value of $T_{d}$ to 0.0075 . Hence, it can be said that, the threshold $T_{d}$ can be chosen in such a way that the size of retrieved list approximately amounts to $10 \%$ of the scene keypoints. This reduces the search space by $90 \%$, removes false positive and eases the job of the 3D descriptor. Moreover another direct implication from reducing the search space by $90 \%$ is the reduction of the computational time required for descriptor matching.

It may seem from Table 5.1 that at $T_{d}=0.0075$, the $\%$ of retrieved lists with groundtruth (recall) for Random Views and Queens datasets is lower, however, the results of descriptor evaluation, as shown later, highlight that only a few matches are found even by the state-ofthe-art descriptors. For example, on the Queens dataset, with $T_{d}=0.0075$, the $\%$ of retrieved lists with groundtruth is $28 \%$, which means that with an ideal descriptor, the highest achievable recall would be 0.28 . However, the best recall achieved by the state-of-the-art/proposed descriptor was just $\approx 0.04$, as shown in Fig. 5.4. This means that, there is lot of scope for improvement in the design of descriptors.

Another main reason behind Queens and other datasets having relatively less \% of retrieved lists with groundtruth is that, the detected keypoints can lie on planar or slightly curved regions 
of the point clouds. In such scenarios, it is hard to capture the 3D surface characteristics as it is not constrained in $x, y, z$ directions. Moreover, Queens dataset has noisy point cloud data with low resolution and the inability of the local reference frame to accurately characterize or capture the nature of $3 \mathrm{D}$ surface patch is another reason that effects the $\%$ of the retrieved lists that contain groundtruth (recall). The performance of the 3D descriptors varies with the nature of the point cloud data as can be seen by the distinctly varying performance on various datasets. Essentially, 3D descriptors are designed for the same purpose, i.e., to capture the characteristics of $3 \mathrm{D}$ surface patch in a unique way and in this work, we propose a very fast way through which the search space can be drastically reduced, thereby enabling the design of simple and fast 3D descriptors that offer good performance.

\subsubsection{DHoPD Descriptor Evaluation}

We compare the proposed 3DHoPD with the state-of-the-art 3D descriptors on four publicly available datasets, as mentioned above, using precision recall curves.

\subsubsection{Steps to calculate Precision-Recall Curves:}

Firstly, a set of keypoints are detected on a scene and those detected scene keypoints are transformed onto the model with the available groundtruth. In this way, all the possible false positives that may arise from the background and clutter from the scene are also accounted for. Next, we extract the considered 3D feature descriptors at these 3D keypoints on both the scene and the model. For every model keypoint's descriptor, we find the first and the second nearest scene keypoint's descriptor based on Euclidean distance metric. If the ratio of the Euclidean distance of first to the second nearest neighbour is less than or equal to a threshold $\alpha$, then it is considered as a correspondence $C_{r}$. The threshold $\alpha$ is varied from 0 to 1 and accordingly the number of correspondences $C_{r \alpha}$ on the considered scene-model pair at various values of $\alpha$ is estimated.

Specifically, we set the values of $\alpha=\{0.2,0.4,0.6,0.75,0.85,0.925,0.95,0.975,1.0\}$ and calculate the correspondences $C_{r \alpha}$. The groundtruth matches $G_{m}$ is equal to the number of model keypoints because, for every model keypoint there lies a corresponding scene keypoint, which can be found via the available groundtruth. The true correspondences True corr $_{\text {are }}$ the correspondences from the set of $C_{r \alpha}$ that align with the groundtruth. Specifically, a correspondence in $C_{r \alpha}$ is considered as a true correspondence $T_{c o r r}$, if the matched scene keypoint lies within a threshold $\epsilon$ to the transformed model keypoint based on the groundtruth. Finally precision and recall at a specific $\alpha$ is calculated as shown below: 


$$
\text { Precision }=\frac{\text { True }_{\text {corr }}}{C_{r \alpha}} \quad \text { Recall }=\frac{\text { True }_{\text {corr }}}{G_{m}}
$$

where $G_{m}$ is the number the groundtruth matches, True corr $_{r}$ represents true correspondences and $C_{r \alpha}$ represents the correspondences that pass the nearest neighbour based ratio test at the considered $\alpha$. Then, the precision and the recall values at a specific $\alpha$ is the averaged value estimated over all scene-model pairs in the considered dataset.

Parameters: ISS keypoint detector was employed for keypoint detection as it offered good keypoint repeatability [147] and efficient keypoint matching performance when combined with various 3D feature descriptors [64]. All the considered 3D feature descriptors are extracted with a support size of $0.06 \mathrm{~m}$ around the detected ISS keypoints. In the case of 3DHoPD, $T_{d}$ as mentioned in Sec. 5.2.2 is set to $0.0075 \mathrm{~m}$. The following are the parameters used for ISS keypoint detection which is publicly available from PCL [126]: model resolution $m r=0.0015 m$, iss_gamma_21 $=i s s \_g a m m a \_32=0.8$, salient_radius $=10 \times$ mr,non_max_radius $=$ $6 \times m r$, normal_radius $=6 \times m r$ and border_radius $=2 \times m r$.

\subsubsection{Comparative Evaluation of 3DHoPD}

The proposed 3DHoPD is compared against state-of-the-art descriptors, SHOT [128], USC [143], FPFH [124] and 3DSC [57]. Each descriptor has varied dimensionality (memory footprint) and accordingly require varied computational time for descriptor matching at full dimensional settings. As the proposed 3DHoPD is significantly lower in dimensionality and in order to have a fair comparison, we create low dimensional representations of the considered SHOT, FPFH, USC and 3DSC descriptors using Principal Component Analysis (PCA) as described in our previous work [110].

In order to learn the PCA transformation matrix for a specific 3D descriptor, we extract

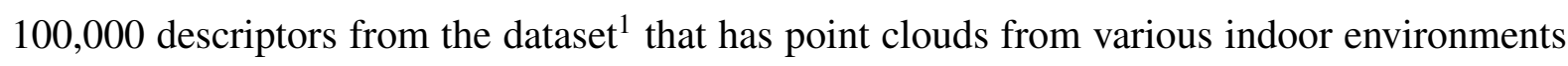
and perform PCA on them. These learnt PCA transformation matrices are then used to create 18-dim variants represented as PCA-SHOT, PCA-FPFH, PCA-USC and PCA-3DSC descriptors. All the learnt PCA transformation matrices will be made publicly available through the project website. Our previous work [110] shows that, after PCA based dimensionality reduction, about $70 \%$ of SHOT descriptors's performance is retained in the first 20 dimensions while $95 \%$ of FPFH descriptor's performance is retained in the first 20 dimensions, of their low dimensional representations. We also present the performance of 15-dim HoPD descriptor to show the enhancement offered by the proposed '3D' keypoint transformation.

\footnotetext{
${ }^{1}$ https://sites.google.com/site/3dkeypoints/
} 


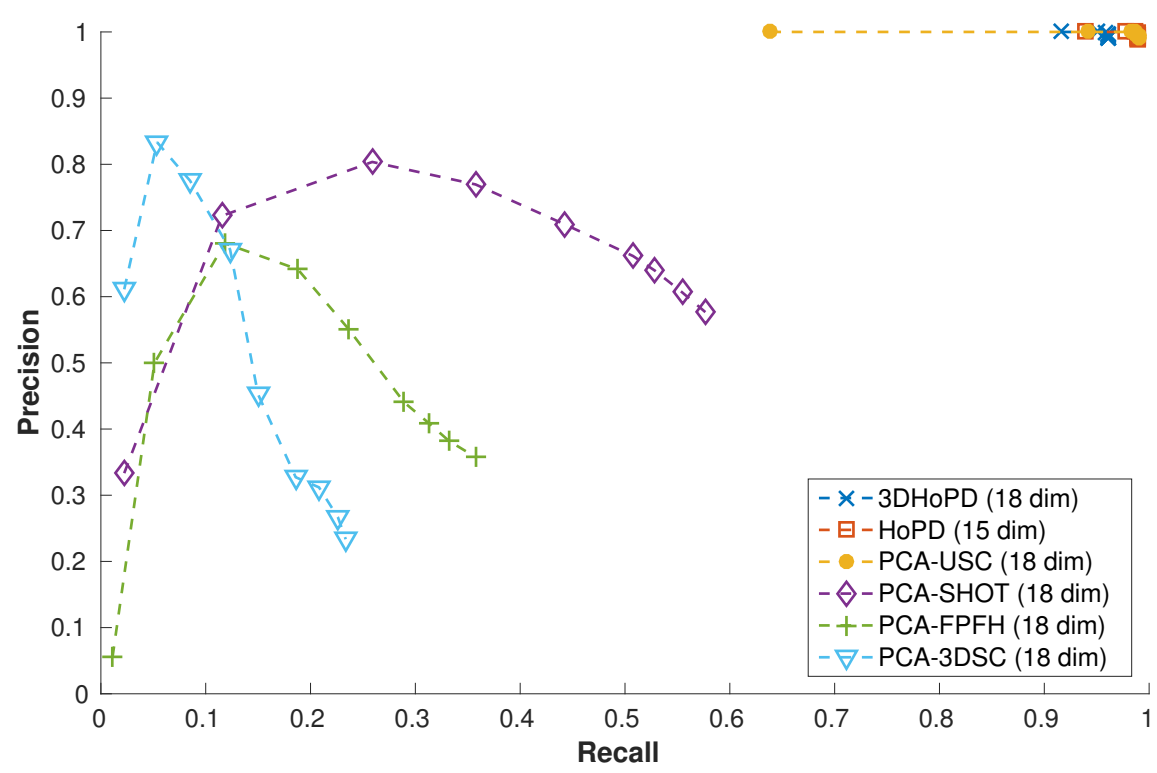

(a) Retrieval dataset with Gaussian noise of $\sigma=0.1 \mathrm{mr}$

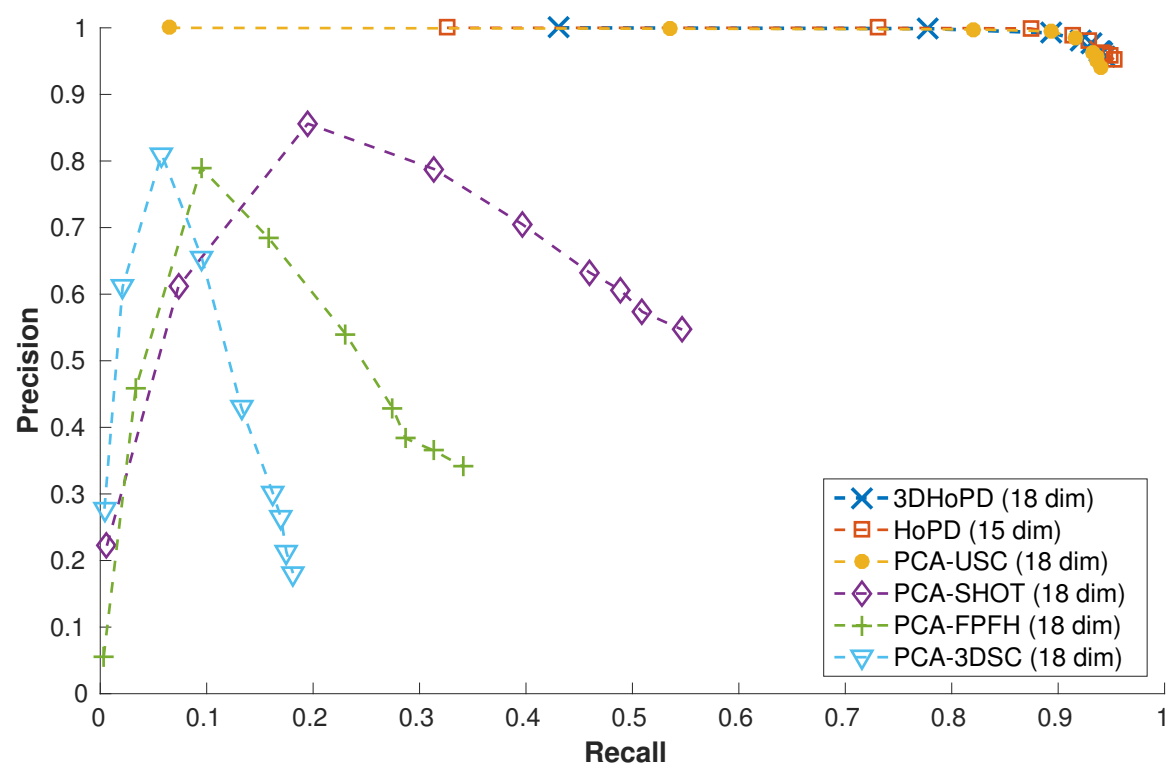

(b) Retrieval dataset with Gaussian noise of $\sigma=0.5 \mathrm{mr}$

Figure 5.2: Performance on Retrieval dataset with varying amount of Gaussian noise with standard deviation $\sigma=\{0.1,0.5\} \mathrm{mr}$.

Performance on Retrieval dataset: Here, we evaluate the performance of 3DHoPD and the considered state-of-the-art 3D descriptors on Retrieval dataset with varying amount of Gaussian noise added on to them. In this dataset, the model and the scene point clouds are the same, however, the scene point clouds are corrupted with noise. Fig. 5.2(a) shows the performance when Gaussian noise with a standard deviation of $0.1 \mathrm{mr}$ (mesh resolution) is added while in Fig. 5.2(b), Gaussian noise with $\sigma=0.5 \mathrm{mr}$ is added. It can be seen from Fig. 5.2(a) 
\& (b) that HoPD, 3DHoPD and PCA-USC offer state-of-the-art performance.

Secondly, a closer look at Fig. 5.2(b) shows that 3DHoPD offers greater precision and recall at a particular $\alpha$ when compared to PCA-USC $(18 \mathrm{dim})$. Please note that the first marker on each precision-recall curve in the figure refers to $\alpha=0.2$ and Fig. 5.2(a) shows that 3DHoPD starts with a recall of $\approx 0.9$ while PCA-USC ( $18 \mathrm{dim}$ ) starts with a recall of $\approx 0.65$. The same observation can be made from Fig. 5.2(b) that 3DHoPD starts with a recall of $\approx 0.45$ while PCA-USC(18 dim) starts with a recall of $\approx 0.1$ at the same value of $\alpha=0.2$. This high recall of 3DHoPD at lower $\alpha$ validates the claim that a list of probable matches with high recall is first generated (by transforming the 3D keypoints to a new 3D space) and then the exact match is later found with HoPD descriptor, offering state-of-the-art performance.

The reasons behind HoPD and 3DHoPD offering nearly same performance is because in this dataset, the scene and the model point clouds are exactly the same, except that the scenes are corrupted with noise, which can be very well captured by the proposed 15-dim HoPD descriptor. However, in the following experiments on other datasets that cater for occlusion and background clutter, the enhancement offered by the proposed 3D keypoint transformation technique stands out significantly by boosting the performance of HoPD.

Performance on Random Views dataset: Fig. 5.3(a) \& Fig. 5.3(b) show the performance of 3DHoPD, HoPD and the low dimensional state-of-the-art descriptors on the Random Views dataset with two settings, where the scenes are corrupted with Gaussian noise of $\sigma=\{0.1,0.5\} \mathrm{mr}$ (mesh resolution). Fig. 5.3(a) shows that for $\alpha>0.925$, 3DHoPD offers both high precision and recall compared to all other descriptors. For descriptor based keypoint matching, having a high recall is preferable because RANSAC can later be used to improve the precision by removing the false positive correspondences. Moreover, $\alpha=1$ boils down to nearest neighbour association, which is the most preferred type of descriptor matching in practical applications. It can be seen from Fig. 5.3(a) that 3DHoPD offered high precision and recall at the most practical operating point, $\alpha=1$, in real world applications.

In the case with higher amount of Gaussian noise $(\sigma=0.5 \mathrm{mr})$, as shown in Fig. 5.3(b), 3DHoPD offered better precision and recall for $\alpha>0.9$, when compared to others. At a few values of $\alpha$ in both Fig. 5.3(a) \& (b), PCA-3DSC has offered good performance in this dataset, however, it performed poorly on all other datasets. It can be seen from Fig. 5.3(a) \& (b) that 3DHoPD offers much better performance than HoPD, which is because of the proposed 3D keypoint transformation method. As, higher recall is preferred in practical scenarios so that the descriptors can provide more number of true matches, it can be said that 3DHoPD is 


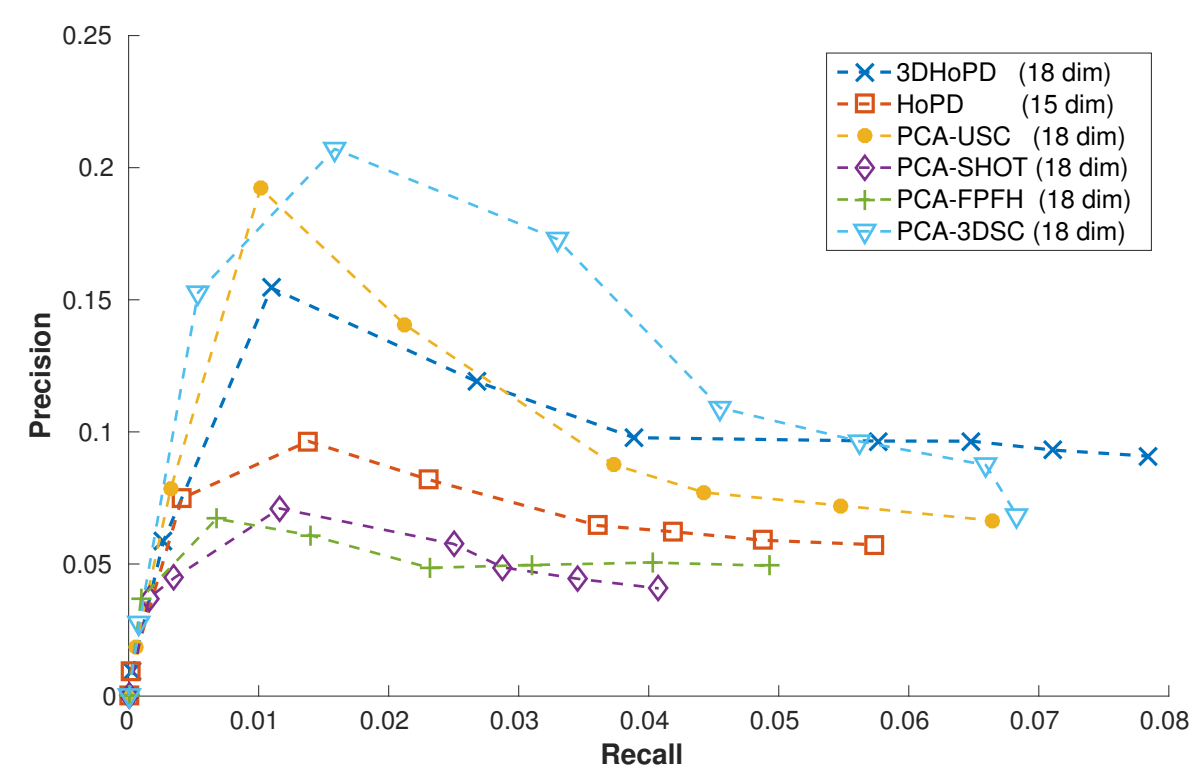

(a) Random Views dataset with Gaussian noise of $\sigma=0.1 \mathrm{mr}$

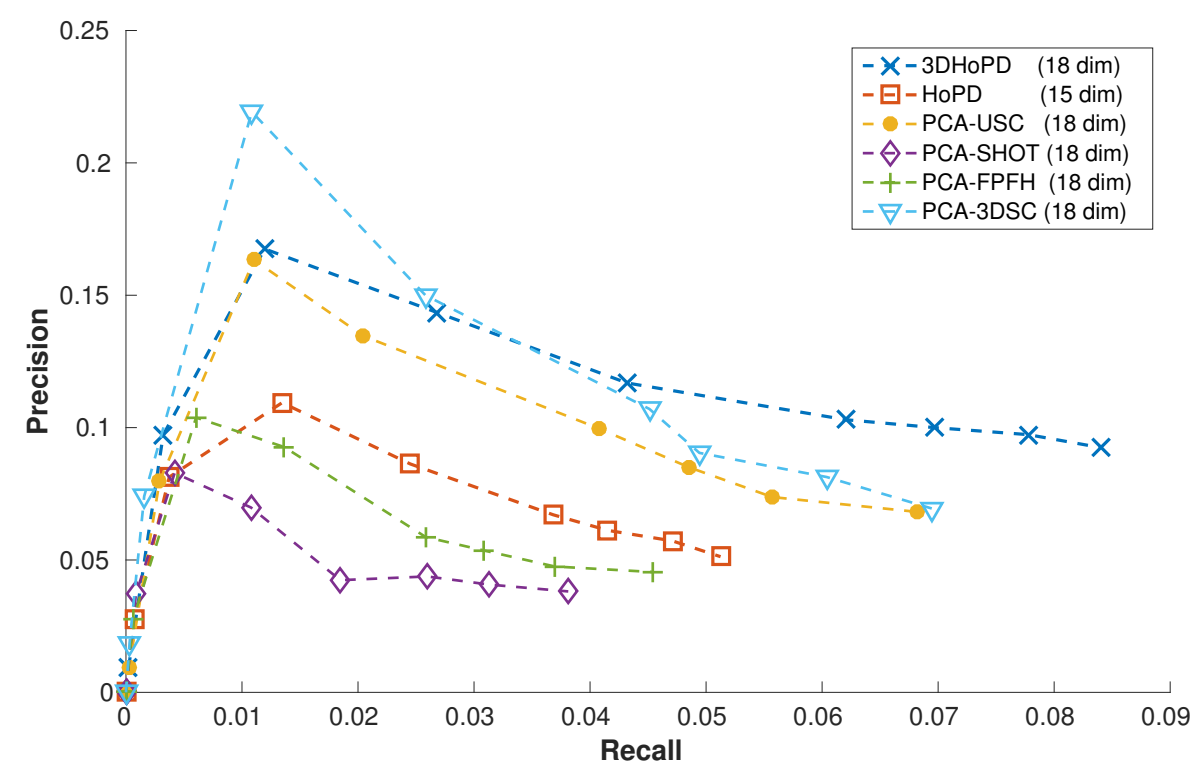

(b) Random Views dataset with Gaussian noise of $\sigma=0.5 \mathrm{mr}$

Figure 5.3: Performance on Random Views dataset with Gaussian noise of standard deviation $\sigma=\{0.1,0.5\} m r$.

more preferable as it consistently offers higher recall across different noise settings showing its dominance over others.

Performance on Queens dataset: Fig. 5.4 shows the performance on Queens dataset and it can be seen that 3DHoPD outperforms all the considered 3D descriptors significantly. This experiment again bolsters the fact that the proposed 3D keypoint transformation significantly enhances the performance of HoPD, as can be seen by the difference in performance 


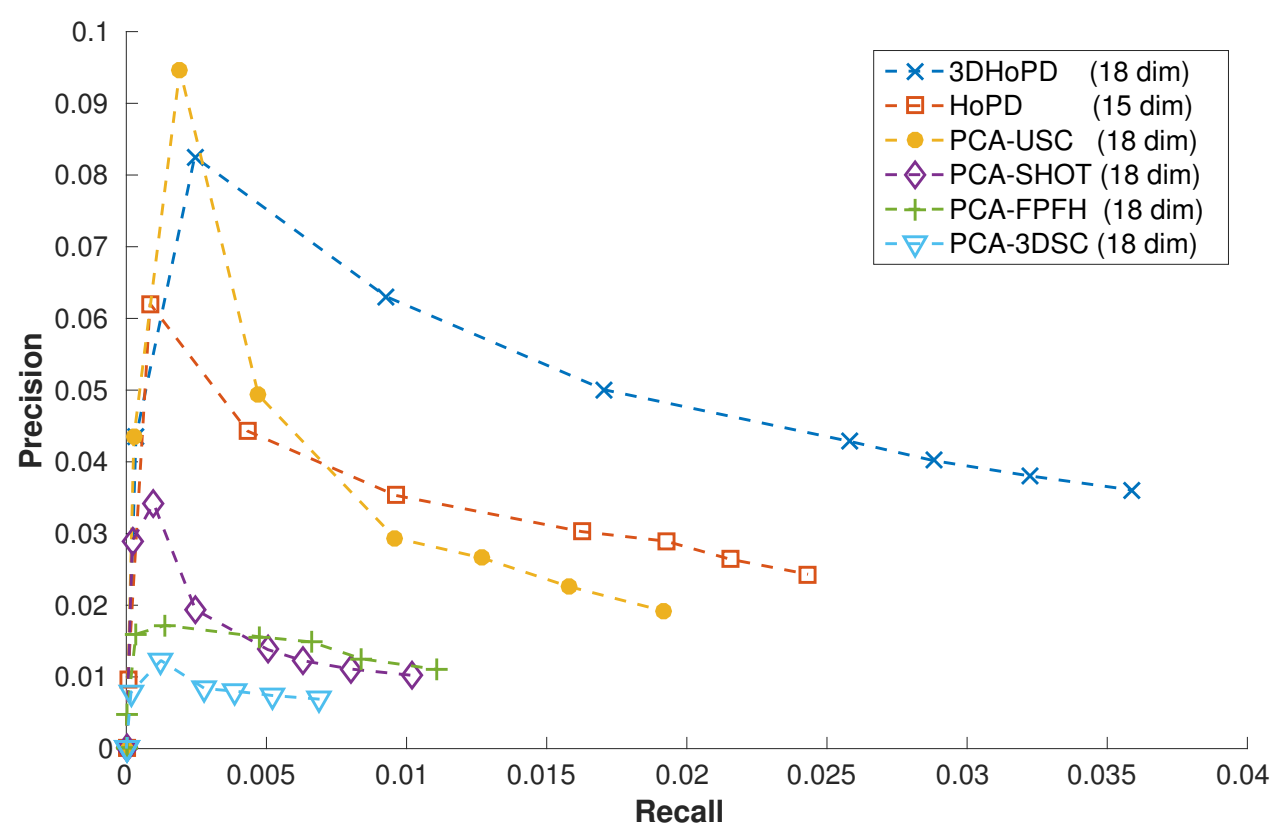

Figure 5.4: Performance on Queens dataset

of 3DHoPD and HoPD descriptors in Fig. 5.4. PCA-USC offers the third best performance followed by PCA-FPFH, PCA-SHOT and PCA-3DSC.

Performance on Kinect dataset: It was observed that the original Kinect dataset does not have significant rotation between the models and the scenes. Hence to accommodate and evaluate the rotational invariance of the considered 3D descriptors, we applied a random 3D transformation between each model-scene pair and then performed the experiments. We also present the performance comparison with full dimensional USC, SHOT, FPFH and 3DSC descriptors for this dataset, whereas the results for other datasets are available on the project website. It can be seen from Fig. 5.5 that full dimensional SHOT, USC and FPFH offer better performance than 3DHoPD. However, please note the huge dimensionality of USC (1980 $\mathrm{dim}$ ) and SHOT (352 dim) while compared to 3DHoPD (18 dim). Next, FPFH requires nearly $275 x$ more computational resources than 3DHoPD, as shown in Sec. 5.3.2.3. While comparing 3DHoPD with 18-dim PCA equivalents, then 3DHoPD comes after PCA-SHOT and PCA-FPFH. PCA-SHOT's and PCA-FPFH's success can firstly be attributed to their design which captures the variations in surface normals and secondly, to the specific characteristic of this dataset for having less surface variations that get accurately captured by surface normals rather than spatial point distributions. However, these PCA-SHOT and PCA-FPFH offer significantly lower performance than 3DHoPD in other datasets while 3DHoPD offers most stable performance among all the considered 3D descriptors across all the datasets. 


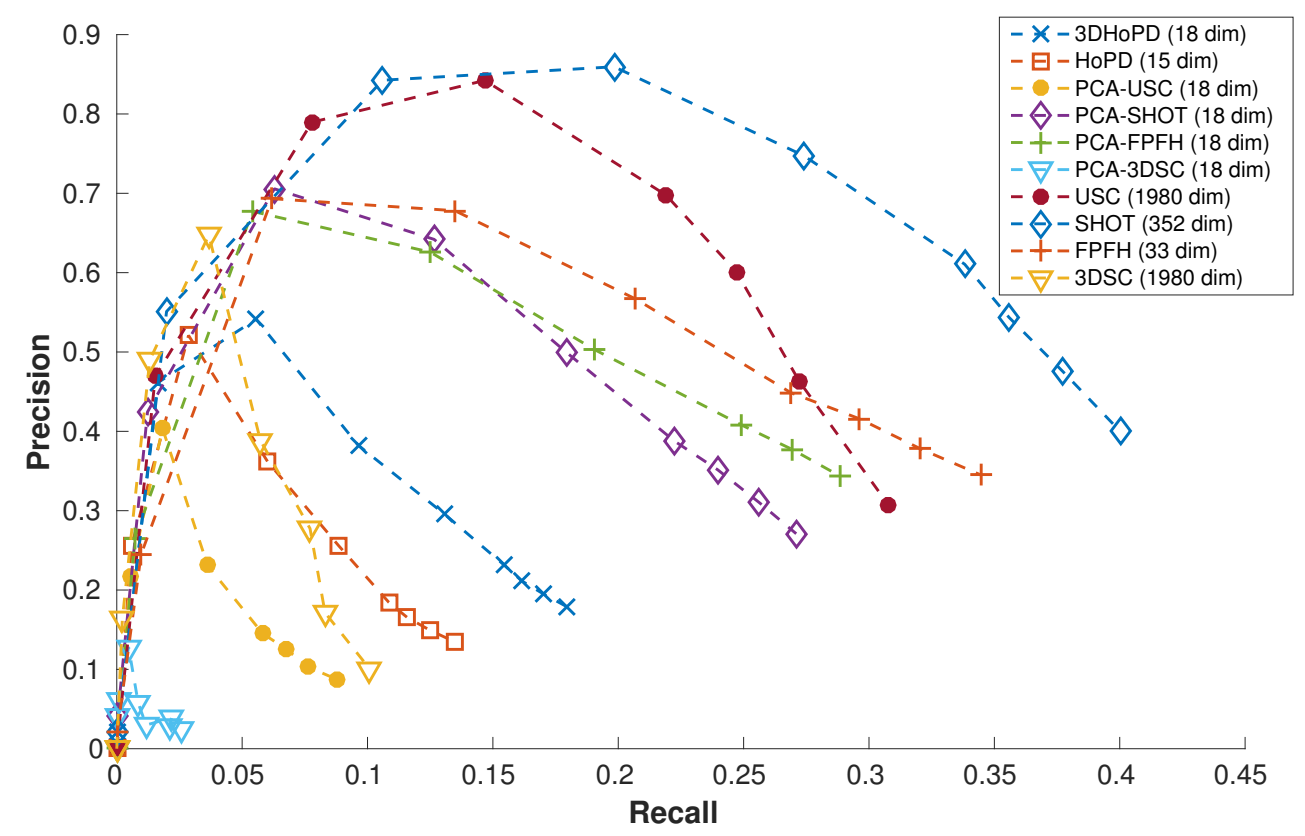

Figure 5.5: Performance on Kinect dataset

Table 5.2: Average number of keypoints and the computational time (in seconds) required for extracting feature descriptors around those keypoints on the Kinect dataset.

\begin{tabular}{cccccc}
\hline \hline No. of Keypoints & 3 DHoPD & USC & SHOT & $3 D S C$ & FPFH \\
\hline 1342 & $\mathbf{1 . 1 2} \mathbf{~ s e c}$ & 9.48 & 14.52 & 25.17 & 276.12 \\
\hline
\end{tabular}

\subsubsection{Computational Requirements For Descriptor Extraction}

We employed a CPU with an Intel Xeon(R) CPU E5-16500@3.20GHz $\times 12$ and 16 GB RAM with $U B U N T U 14.04$ operating system. The ISS keypoints were detected and 3D descriptors were extracted with exactly same settings as in previous experiments on the Kinect dataset, which has a mesh resolution of $\approx 0.001 \mathrm{~m}$. Table 6.3 reports the average number of keypoints detected and the time taken to extract 3D descriptors around them. It can be seen that to extract 1342 descriptors, 3DHoPD requires just $1.12 \mathrm{sec}$ (10x faster) while all others require significantly higher computational time. The average number of points in the local patch used for descriptor extraction in our experiments turned out to be 12300. The high computational requirements of FPFH descriptor can be justified from the survey paper [64], which mentions that FPFH is only efficient when the number of points are lower than 5000 and its computational requirements increase later on.

Also note that for other descriptors, we did not account the time taken to create low dimensional representations (through PCA transformation matrices) and only reported the time taken 
to extract full dimensional feature descriptors. 3DHoPD has a great computational edge over other descriptors as it does not involve expensive surface normal estimation. This shows that 3DHoPD stands out from the existing 3D descriptors in terms of fast descriptor extraction, low memory footprint and stable performance across various datasets.

Memory footprint: According to standard memory allocation in $\mathrm{C} / \mathrm{C}++$ standards, the proposed 18 dimensional 3DHoPD requires $18 * 4=72$ bytes of memory to store a single descriptor. This is significantly lower when compared to the widely used 352 dimensional SHOT descriptor, which requires $352 * 4=1408$ bytes of memory for a single SHOT descriptor.

\subsection{Observations and Discussions}

Firstly, USC, SHOT and the proposed 3DHoPD descriptors employ the same technique to estimate the local reference frame and then transform the $3 \mathrm{D}$ surface patch accordingly to achieve rotational invariance.

Secondly, 3DSC and USC descriptors create huge number of partitions (1980) in the superimposed spherical grid and represent each grid with just a single value calculated from the weighted sum of points that fall in each partition. In this way, they lose out on actual surface variations along $x, y, z$ directions by only accounting for the 'number' of points present in each partition, which can be reason for their low performance. SHOT descriptor has less number of partitions compared to USC, however, it represents each partition with a histogram of normals, which does capture low level surface variations as observed in the Kinect dataset, however, coarse partitioning of spherical grid and not accurately accounting for the number of points is the reason for its low performance on other datasets.

Thirdly, FPFH strongly relies on normals for Darbaux frame computation and hence offers good performance on Kinect dataset but it loses out on other datasets as it does not directly and accurately account for spatial distributions and variations of the surface points along $x, y, z$ directions. Moreover, high computational requirements of FPFH descriptor on dense point clouds is a huge drawback.

Our previous work [110] shows that, after PCA based dimensionality reduction, about $70 \%$ of SHOT descriptors's performance is retained in the first 20 dimensions while $95 \%$ of FPFH descriptor's performance is retained in the first 20 dimensions, of their low dimensional representations. Hence, dimensionality reduction also accounts for the loss in performance of the descriptors on these datasets. 
Next, the competitive and the stable performance of 3DHoPD across multiple datasets can be strongly attributed to the proposed 3D keypoint transformation to a new 3D space from which a small list of highly probable matches can be retrieved, effectively getting rid of large number of false positives and hence making the job for descriptor matching easier.

Finally, HoPD actually considers the surface variations along and $x, y, z$ directions and also accounts for the number of points through histogram normalization. However, histogram based binning does not account for little surface variations (which normals are good at) and hence offers reduced performance in the Kinect dataset. 3DHoPD's stable performance across datasets and its very low memory and computational requirements, compared to other descriptors, makes it a preferable choice in mobile applications.

\subsection{Conclusion}

A new low dimensional (18-dim) 3D descriptor, 3DHoPD, that is extremely fast to compute is introduced in this chapter. It has less memory footprint and low computational requirements. A new concept, 3D keypoint transformation technique is proposed, in which, the 3D keypoints are transformed to a new 3D space, where keypoints arising from similar 3D surface patches lie close to each other. Capitalizing on this idea, for a given source keypoint, a list of probable target keypoint matches are retrieved with high recall, effectively reducing the search space by $90 \%$. Then, histogram of point distributions (HoPD) is proposed to find an exact correspondence from the found list of probable matches. Experiments have showed that 3DHoPD offers stable and competitive performance across multiple datasets and has dramatically low computational costs, when compared to existing state-of-the-art 3D descriptors at similar dimensionality.

This chapter presented a new low dimensional 3D descriptor '3DHoPD', that has both low memory and computational footprints. However, it is still a real valued 3D descriptor. In 2D image domain, binary descriptors are heavily being researched because they require single bit for each dimension and they can be matched extremely fast using Hamming distance metric, which is equivalent to XOR operation. When the target application requires storage and matching of thousands of descriptors, then the memory and computational gains offered by binary descriptors become very significant. Hence, in the next chapter, we present the first attempt in creating a binary $3 \mathrm{D}$ feature descriptor. 


\section{Chapter 6}

\section{B-SHOT: A Binary 3D Feature Descriptor}

\subsection{Introduction}

In the previous chapter, we presented yet an other real valued 3D feature descriptor, 3DHoPD. Compared to existing state-of-the-art real valued descriptors, 3DHoPD, has both low dimensionality and extraction complexity. In a real valued descriptor, each value has a floating point representation. A floating point value is allocated 4 bytes of memory or 32 bits based on IEEE floating point standards. Real valued feature descriptors are generally matched by calculating Euclidean distance in their high dimensional vector spaces and this is a computationally expensive step. Moreover, the computational requirements for feature descriptor matching and the memory footprint increases proportionally with the increase in the dimensionality of the feature descriptor.

To develop mobile applications that require less memory and lower computational power, binary feature descriptors $[19,141,4,85,18,149]$ have been heavily researched and developed in 2D image domain. They are extensively used in various applications such as $2 \mathrm{D}$ object detection/tracking, image retrieval [163], visual odometry [86] and place recognition [59] in SLAM for robotics. Binary feature descriptors win over traditional real valued feature descriptors as they can be matched extremely fast via Hamming distance metric and have dramatically less memory footprint. Therefore, in this chapter, we present the first attempt in literature in creating a binary 3D feature descriptor for keypoint point matching on 3D point clouds.

Why do we need binary 3D feature descriptors?

Recently, there has been a surge of consumer applications that employ 3D depth sensors and process dense depth data on mobile devices such as Google Tango [1] and Intel RealSense camera. With the availability of these mobile 3D data acquisition devices, there is a need to develop applications that have a low memory footprint and require less computational power. 
Moreover, with the development of hand-held 3D scanning algorithms, such as KinectFusion [103] and CopyMe3D [139] [27], various applications such as, 3D object recognition [63, 8] and 3D shape retrieval [142] are gaining a lot of research attention. These factors necessitate the development of binary feature descriptors in 3D domain as well to develop applications for memory and power constrained devices. Apart from reducing memory footprint and computational costs, a 3D binary feature descriptor can be used to aid 2D feature descriptor matching to achieve much higher accuracy.

\section{Challenges in developing binary 3D feature descriptors from raw point clouds:}

Though there are quite a few binary feature descriptors in the $2 \mathrm{D}$ image domain, there are no binary feature descriptors in the 3D point cloud domain. There are three main reasons for this. Firstly, there is no attribute in $3 \mathrm{D}$ point cloud data, $[x y z]$, that is equivalent to the pixel intensity in 2D image domain. Secondly, 3D data from affordable sensors like Microsoft Kinect is quite noisy, hence simple thresholding, which is often used in 2D binary feature descriptors, turns out to be ineffective. Lastly, the 3D point cloud data can be unordered, i.e., neighbourhood connectivity information may not be present, whereas 2D images are ordered.

Contribution: As it is challenging to directly create a binary 3D descriptor from raw point clouds for the above mentioned reasons, we propose a new binarization technique and apply it on a state-of-the-art 3D feature descriptor, SHOT [128], to create its binary counterpart, BSHOT. The advantages of B-SHOT over the traditional SHOT feature descriptor are as follows. Firstly, SHOT, which is of 352 dimensions (requiring 1408 bytes) is reduced to 352 bits of binary data, therefore reducing the memory footprint, by 32 times. Secondly, keypoint matching can be performed much faster with Hamming distance metric when compared to real valued 3D feature descriptors (Experiments show a $6 \mathrm{x}$ improvement). Then, we propose an evaluation metric for 3D feature descriptor evaluation that considers 3D keypoint detection ambiguity and all possible false feature correspondences. A comprehensive evaluation on benchmark datasets shows that B-SHOT offers comparable keypoint matching performance to that of the state-ofthe-art 3D feature descriptors with added advantages of having low memory and computational requirements.

\subsection{Related Work}

Existing real valued 3D feature descriptors were reviewed in Chapter 2 and in Sec. 2.5.2, the construction principle behind SHOT [128], which is extensively used throughout this chapter is also presented. Here, in this section, we present an overview of 2D binary feature descriptors. 


\subsubsection{D Binary Feature Descriptors}

Coming to the binary feature descriptors in the 2D image domain, most of them $[19,85,4]$ are constructed by comparing the intensity values of pixels that lie in the neighbourhood/support region. They vary in terms of the sampling pattern employed for comparison. BRIEF [19] compares the intensity values of the center pixel with another being chosen from an isotropic Gaussian distribution. FREAK [4] and BRISK [85] use sampling patterns of overlapping concentric circles and differ in the number of points that lie in inner and outer rings. It is not straightforward to extend the pixel based thresholding methods [19, 4, 85] employed in the 2D image domain to 3D point clouds as there is no attribute in 3D point clouds that is equivalent to pixel intensity in 2D images and the 3D point clouds can be unordered.

There are other works that employ hashing [18, 9, 127], learning based approaches [149, $157,61]$ to create 2D binary feature descriptors as well. Recently, deep learning [82, 83, 77], capitalizing on lot of labelled training data has been instrumental in transforming $2 \mathrm{D}$ image domain. It was shown that the pre-trained deep convolutional neural networks models can be fine tuned to achieved state-of-the-art image segmentation $[89,88]$ and object detection [60, 117, 69]. Following these lines, a 2D binary descriptor called DeepBit [87] and others $[115,159,52]$ has been proposed to learn compact binary patch descriptors, as a replacement to SIFT. However, the lack of standard and publicly available 3D data for training purposes, the difference that arises from $2.5 \mathrm{D}$ and $3 \mathrm{D}$ depth data and point cloud density variations make it difficult to import these learning based techniques. Hence, we propose a binarization method that is data-independent and do not require any learning, to convert a real valued 3D feature descriptor into a binary descriptor for fast keypoint matching on 3D point clouds.

There are other class of works in 2D image domain which binarize the real valued 2D feature descriptors, such as SIFT [90], to create a binary feature descriptor. B-SIFT [163] calculates the median of a considered SIFT feature descriptor, assigns a binary value of ' 1 ' if the descriptor value is greater than the calculated median and ' 0 ' otherwise, which results in a 128 bit binary SIFT descriptor. They also extend it to a 256 bit descriptor by thresholding with two medians taken from the considered SIFT feature descriptor. Dominant-SIFT [141] uses the binary coded position of the cumulative maximum value in each sub-histogram of the SIFT feature descriptor to create a 48-bit binary feature descriptor.

We take inspiration from B-SIFT [163] and Dominant-SIFT [141] algorithms in proposing our binarization technique to convert a real valued feature descriptor to a binary feature descriptor. Our experiments showed that the Dominant - SIFT's binarization technique performed very 
poorly on SHOT feature descriptor. There are two reasons, firstly, the encoded binary position of the maximum value in each sub-histogram can vary significantly in noisy 3D data [64, 128]. Secondly, many feature descriptors can have exactly same binary representation (in the case of Dominant-SIFT) [141], resulting in many false correspondences. Our proposed binarization scheme is adaptive and stores more information thereby resulting in better performance when compared to B-SIFT's quantization scheme. Moreover, when we applied our binarization technique to SIFT in the 2D domain, it offered superior performance to Dominant SIFT [141] and B-SIFT [163] on benchmarks proposed by Mikolajczyk et al. [97]. However, here, we confine ourselves to evaluating the proposed binarization technique comprehensively on $3 \mathrm{D}$ feature descriptors alone.

Chapter Organization: In Sec. 6.3, we explain the proposed technique to binarize SHOT to create B-SHOT. Later, in Sec. 6.4.2, we apply the proposed binarization technique on SHOT, RoPS and FPFH to create B-SHOT, B-RoPS and B-FPFH. Then based on three experiments, first one based on precision recall curves, second one based on distance distributions and third one based on true keypoint correspondences, it is shown that B-SHOT outperforms B-RoPS and B-FPFH. In other words, the proposed technique works well with SHOT compared to others such as RoPS and FPFH. Then, in Sec. 6.4.3, we present the drawbacks of Recognition Rate metric, enhance it and propose Robust Recognition Rate (RRR)metric. In Sec. 6.4.4, we compare B-SHOT with SHOT, RoPS and FPFH descriptors using $R R R$ metric. Later, in Sec. 6.5, parameter evaluation of two free parameters in the proposed binarization technique is performed. Lastly, in Sec. 6.6, the computational and memory requirements are presented.

\subsection{The Proposed Technique to Binarize SHOT}

The SHOT feature descriptor is a 352 dimensional vector ${ }^{1}$ comprising of 11 bin histograms arising from 32 spatial grids in 3D space. Each histogram represents the angles that the surface normals in a certain spatial grid make with the local reference frame at the considered keypoint. To create a B-SHOT feature descriptor, we encode the 352 dimensional SHOT descriptor into a 352 bit binary descriptor. Let us consider a SHOT descriptor $S_{i}$, where $i=\{0,1,2, . ., 351\}$ and each value of $S_{i}$ can be any decimal value between 0 and 1 . Let us represent the newly created B-SHOT feature descriptor by $B_{i}$, where $i=\{0,1,2, . ., 351\}$ and each value of $B_{i}$ is either 0 or 1 . This binarization of SHOT to B-SHOT is performed in an iterative procedure that

\footnotetext{
${ }^{1}$ We employ the default implementation of SHOT feature descriptor available through Point Cloud Library at www.pointclouds.org
} 
takes ' $m$ ' consecutive values from the beginning of $S_{i}$ and encodes them into corresponding $m$ binary bits in $B_{i}$. In the proposed binarization technique, we roughly preserve the shape of the sub-histograms from various spatial grids that are concatenated to form a SHOT feature descriptor.

As mentioned above, ' $m$ ' represents the number of real values that are encoded into binary bits. For the case where $m=4$, consider four values $\left\{S_{0}, S_{1}, S_{2}, S_{3}\right\}$ from the SHOT feature vector, $S_{i}$, and its corresponding four bits $\left\{B_{0}, B_{1}, B_{2}, B_{3}\right\}$ to be encoded into B-SHOT descriptor, $B_{i}$.

Following are the various possibilities of encoding $S_{i}$ into $B_{i}$ :

Let $S_{\text {sum }}=S_{0}+S_{1}+S_{2}+S_{3}$.

- Case A: If all four values in $S_{i}$ are zeros, then the corresponding four bits of $B_{i}$ are also set to zero, i.e., $\left\{B_{0}, B_{1}, B_{2}, B_{3}\right\}$ will be $\{0,0,0,0\}$ in this case.

- Case B: If Case A does not hold, we check if there is a single value, $S_{i}, S_{i} \in\left\{S_{0}, S_{1}, S_{2}, S_{3}\right\}$ that amounts to $90 \%$ of $S_{\text {sum }}$. If yes, then its position is coded in a binary fashion. For example, if $S_{1}$ 's value amounts to more than $90 \%$ of the $S_{\text {sum }}$, then the encoded $\left\{B_{0}, B_{1}, B_{2}, B_{3}\right\}=\{0,1,0,0\}$. In this way, four cases are covered and the possible values of $\left\{B_{0}, B_{1}, B_{2}, B_{3}\right\}$ are $\{1,0,0,0\},\{0,1,0,0\},\{0,0,1,0\},\{0,0,0,1\}$.

- Case C: If Case A and Case B do not hold, then we check if the sum of any two values amount to $90 \%$ of $S_{\text {sum }}$. For example, if the sum of $S_{0}$ and $S_{3}$ amounts to more than $90 \%$ of $S_{\text {sum }}$, then the encoded $\left\{B_{0}, B_{1}, B_{2}, B_{3}\right\}=\{1,0,0,1\}$. In this way, the possible values of $\left\{B_{0}, B_{1}, B_{2}, B_{3}\right\}$ are $\{1,1,0,0\},\{1,0,1,0\},\{1,0,0,1\},\{0,1,1,0\},\{0,1,0,1\}$ and $\{0,0,1,1\}$. Pseudocode for this case is shown in Algorithm 3.

- Case D: If Case A, Case B and Case C do not hold, then we check if the sum of any three values amounts to more than $90 \%$ of $S_{\text {sum. }}$. The possible values of $\left\{B_{0}, B_{1}, B_{2}, B_{3}\right\}$ in this case turn out to be $\{1,1,1,0\},\{0,1,1,1\},\{1,1,0,1\}$ and $\{1,0,1,1\}$.

- Case E: If none of the above conditions hold, then it means that $\left\{S_{0}, S_{1}, S_{2}, S_{3}\right\}$ are nearly the same values and the encoded $\left\{B_{0}, B_{1}, B_{2}, B_{3}\right\}$ would be $\{1,1,1,1\}$.

We apply the proposed binarization technique on all consecutive $m$ sets in a 352 dimensional SHOT descriptor, i.e., $\left\{S_{0}, S_{1}, S_{2}, S_{3}\right\}$ is encoded as $\left\{B_{0}, B_{1}, B_{2}, B_{3}\right\},\left\{S_{4}, S_{5}, S_{6}, S_{7}\right\}$ is encoded as $\left\{B_{4}, B_{5}, B_{6}, B_{7}\right\}$, and so on, to create a 352 bit binary descriptor, B-SHOT. When 


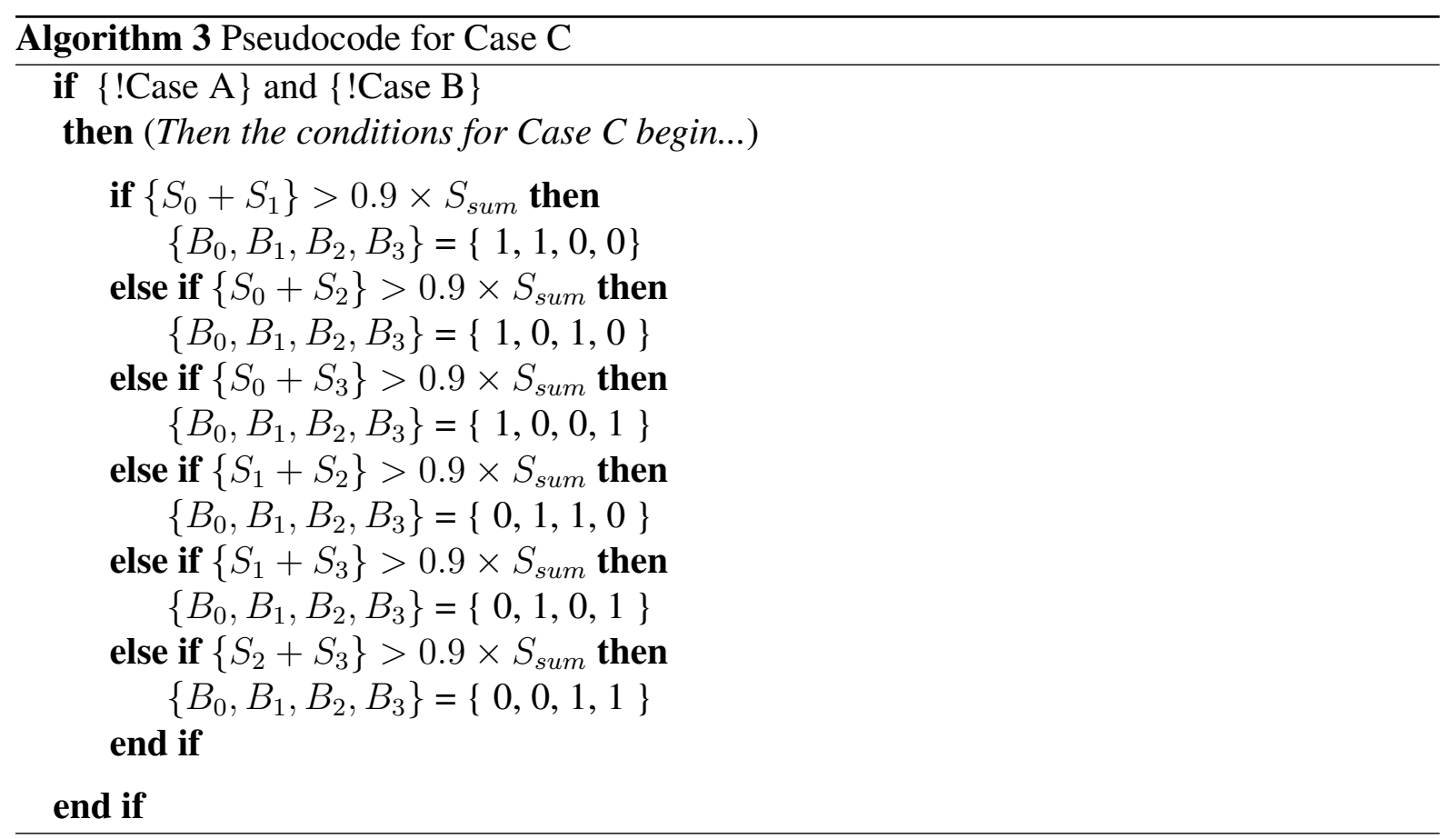

there are multiple possible subcases in Case C and Case D where sum of $\left\{S_{0}, S_{1}, S_{2}, S_{3}\right\}$ add upto $90 \%$ of $S_{\text {sum }}$, then the proposed algorithm chooses the first encountered case to maintain consistency in the binarization scheme.

In the proposed binarization technique, there are two free variables, $m$ (number of values that are being encoded into binary bits) and $E_{r}$ (encoding ratio which was set to 0.9 or $90 \%$ in the above scenario). Depending on the value of $m$, there will be $2^{m}$ conditions to check for, while performing the binarization. This evaluation of $2^{m}$ conditions can be easily simplified by employing a sorting algorithm that sorts the $m$ input real values and then encodes the indices of the minimal set of sorted values that amount to $E_{r} \times S_{\text {sum }}$. We present the pseudocode for this in Algorithm 4. We take $m$ real values as the input and create an array, vector_pair, in which each element is $\left\{S_{i}, i\right\}$. The array, vector_pair is sorted only based on the values of $S_{i}$. Then we iterate through vector_pair and find the first $j$ elements that amount to $90 \%$ of the $S_{\text {sum }}$. To create the binary vector of $m$ values, the corresponding indices of the $j$ elements are set to 1 while others remain 0 .

Intuition behind B-SHOT: The proposed binarization method was developed based on the intuitions gained from the structure and the distribution of SHOT descriptors on various 3D point clouds. The following observations were made from various experiments on different $3 \mathrm{D}$ point clouds, for example, let us consider an indoor scene, a 3D point cloud as shown in the project website ${ }^{2}$, and extract 1000 SHOT descriptors from uniform keypoints on it. Fig. 6.1(a)

\footnotetext{
${ }^{2}$ https://sites.google.com/site/bshotdescriptor/scene-for-shot
} 
Algorithm 4 Pseudocode to create a binary vector from a real valued vector in which there is no need to hard-code any conditions into the program.

Input: $\left\{S_{0}, S_{1}, \ldots, S_{m-1}\right\}$ real values, $E_{r}, m$

Output: Bit $=\left\{B_{0}, B_{1}, \ldots, B_{m-1}\right\}$ binary values.

Initialization: Create a vector_pair with $S_{\text {values }}:\left\{S_{0}, S_{1}, \ldots, S_{m-1}\right\}$ and its index values as vector_pair $=\left\{\left\{S_{0}, 0\right\},\left\{S_{1}, 1\right\}, \ldots,\left\{S_{m-1}, m-1\right\}\right\}$

$S_{\text {sum }}=S_{0}+S_{1}+\ldots+S_{m-1}$

Temporary variables: temp_sum, bit_count, $i, j, k$, check $=0$.

Step 1: Sort the vector_pair only based on the $S_{i}$ values.

\section{Step 2:}

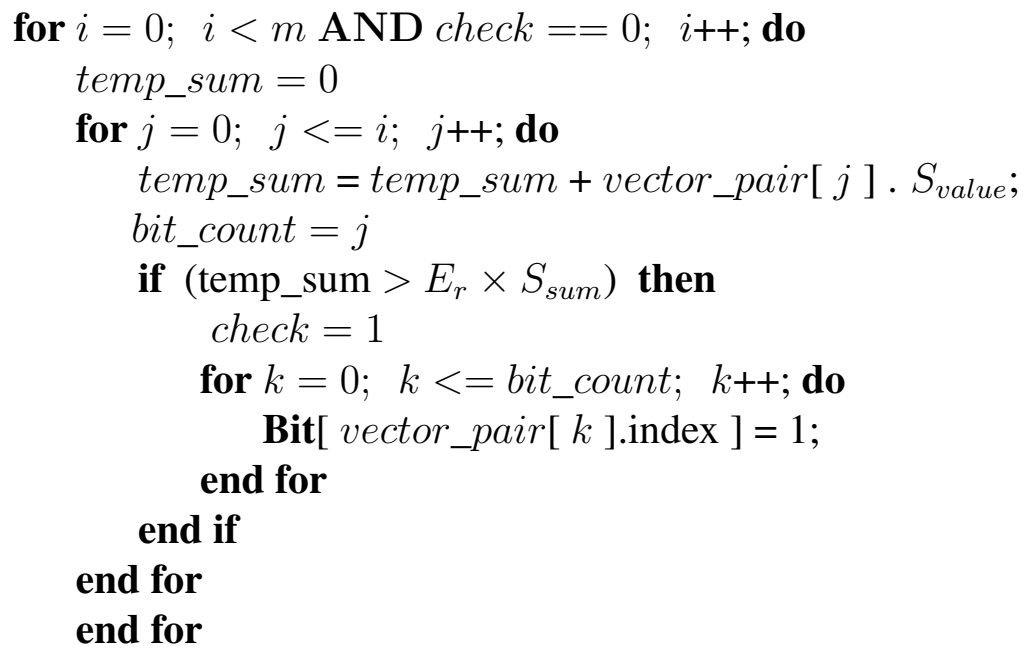

shows the distribution of these 1000 SHOT descriptors. It can be seen from Fig. 6.1(a) that there exists a repetitive pattern in SHOT descriptor's values, and this was the reason behind choosing an iterative method to binarize a chunk of real valued SHOT descriptor to a binary BSHOT. Next, Figs. 6.1(b)(c)\&(d) show three randomly picked SHOT descriptors from the same scene. It can be observed from these figures that there can be peak values in SHOT descriptor with neighbourhood bins going to nearly zero or with nearly same neighbourhood values or they can decrease gradually.

To accommodate all the possible scenarios, the proposed binarization method had Case B, which catered for peaked values, Case C \& Case D, which catered for gradual decrease in the histogram neighbourhood values and lastly Case $\mathrm{E}$ that checked if all the neighbourhood bins are similar. However, still the chunk size $m$ and the encoding ratio $E_{r}$, which catered for the peaked value in the chunk are yet to be determined. Hence, we perform a parameter evaluation experiment by varying these two free parameters, $m$ and $E_{r}$, and show the performance of BSHOT in Sec. 6.5. It was observed from the experiments in Sec. 6.5 that B-SHOT is robust to these two parameter variations, $m$ and $E_{r}$, hence making it reliable. However, with the 


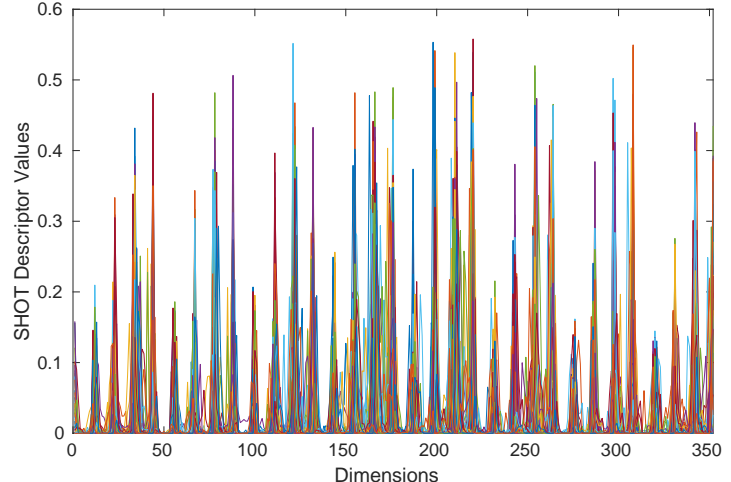

(a)

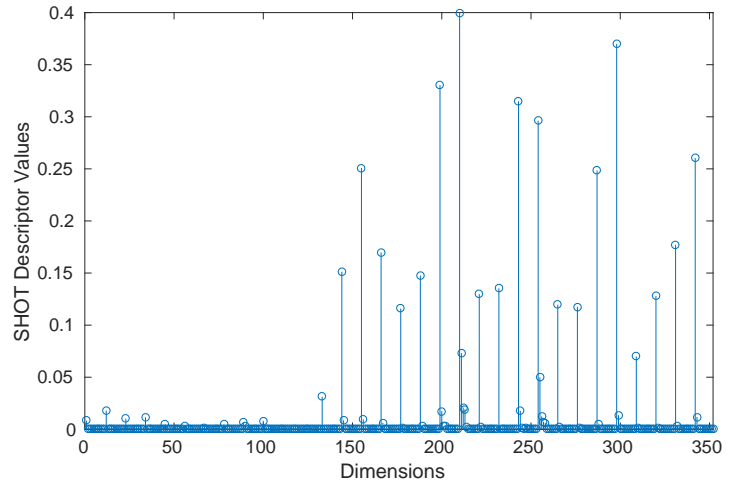

(c)

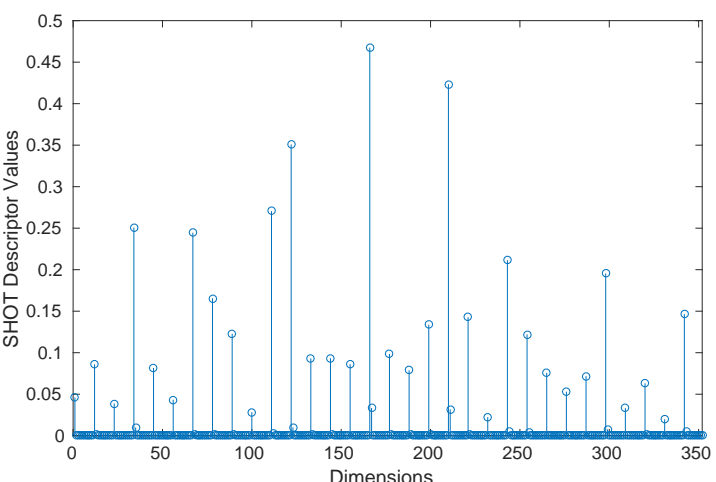

(b)

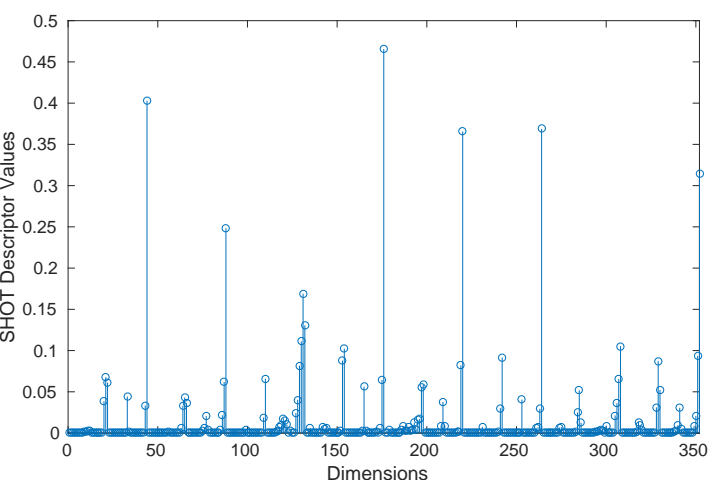

(d)

Figure 6.1: (a) illustrates the distribution of 1000 SHOT feature descriptors from a scene. (b), (c) and (d) shows three randomly picked SHOT descriptors from the scene.

values of $m=4$ and $E_{r}=0.9$, there is a slight better performance and the implementation boils down to a set of 16 'if-else' conditions. Hence we use these settings while evaluating the performance of B-SHOT.

It is important to note that there is loss of information while converting a SHOT feature descriptor into a B-SHOT feature descriptor. Mainly, in Case C and Case D, the individual contributions made by each of the four values of $S_{i}$ are ignored if they sum to $90 \%$ of $S_{\text {sum }}$. For example, let us look at this extreme case, if $\left\{S_{0}, S_{1}, S_{2}, S_{3}\right\}=\{0.65,0.20,0,0\}$, then the encoded $\left\{B_{0}, B_{1}, B_{2}, B_{3}\right\}$ would be $\{1,1,0,0\}$. As can be seen from the above example, the binary representation highlights that bits $B_{0}$ and $B_{1}$ are the same, but in reality they are not. It should be noted that, as a result of information loss and quantization effects in the proposed binarization technique, a slightly lower number of correspondences are found by BSHOT when compared to those found by SHOT. We added more information about the relative largeness of the encoded values into the binary descriptor with few more extra bits, but it did not improve the performance of B-SHOT, highlighting that the current binarization scheme holds the required information in a compact form. The way we added the extra information about the 
relative largeness and the experimental results are available online ${ }^{3}$.

3D feature descriptors are mainly employed to calculate approximate 3D transformation between a source and a target point cloud, which is used to initialize an ICP[13] algorithm that performs fine and accurate registration. Hence, a marginal reduction in the number of correspondences is acceptable if the found correspondences can roughly estimate the 3D transformation matrix.

\subsection{Experimental Evaluation}

In this section, we apply the proposed binarization technique on three state-of-the-art 3D feature descriptors, SHOT, RoPS and FPFH and show that it offers good performance when applied on SHOT descriptor. Later, we provide an extensive performance evaluation of the B-SHOT binary feature descriptor in terms of its capability to estimate $3 \mathrm{D}$ transformation and quantitative evaluation of the established keypoint correspondences while comparing with the state-of-theart real valued 3D feature descriptors.

The source code of B-SHOT and additional experimental results are publicly available at https://sites.google.com/site/bshotdescriptor/

\subsubsection{Experimental Setup}

In all our experiments, we have used a CPU with an Intel Xeon $(R) C P U$ E5-1650 $0 @ 3.20 \mathrm{GHz}$ $\times 12$ and 16 GB RAM with UBUNTU 14.04 operating system.

\subsubsection{Dataset}

We employ the publicly available Kinect dataset ${ }^{4}$ [147] for experimental evaluation because of its practical relevance in real world applications. The relevance of the Kinect dataset to practical applications was also mentioned in [64], as it has inherent noise that arises from the widely used Microsoft Kinect sensor. The Kinect dataset provides models, scenes and respective ground truth 3D transformations between them. In this dataset, models represent the objects whereas scenes contain a collection of various objects in different orientations and occlusions, with a back ground. The ground truth information provides the 3D transformation between a scene and an object model present in the considered scene. Each scene has about 3 models (objects) present in it and there are 49 scene-model samples in total. Hence, we perform extensive

\footnotetext{
${ }^{3}$ http://tinyurl.com/eb-shot

${ }^{4} \mathrm{http} / / /$ vision.deis.unibo.it/research/80-shot
} 

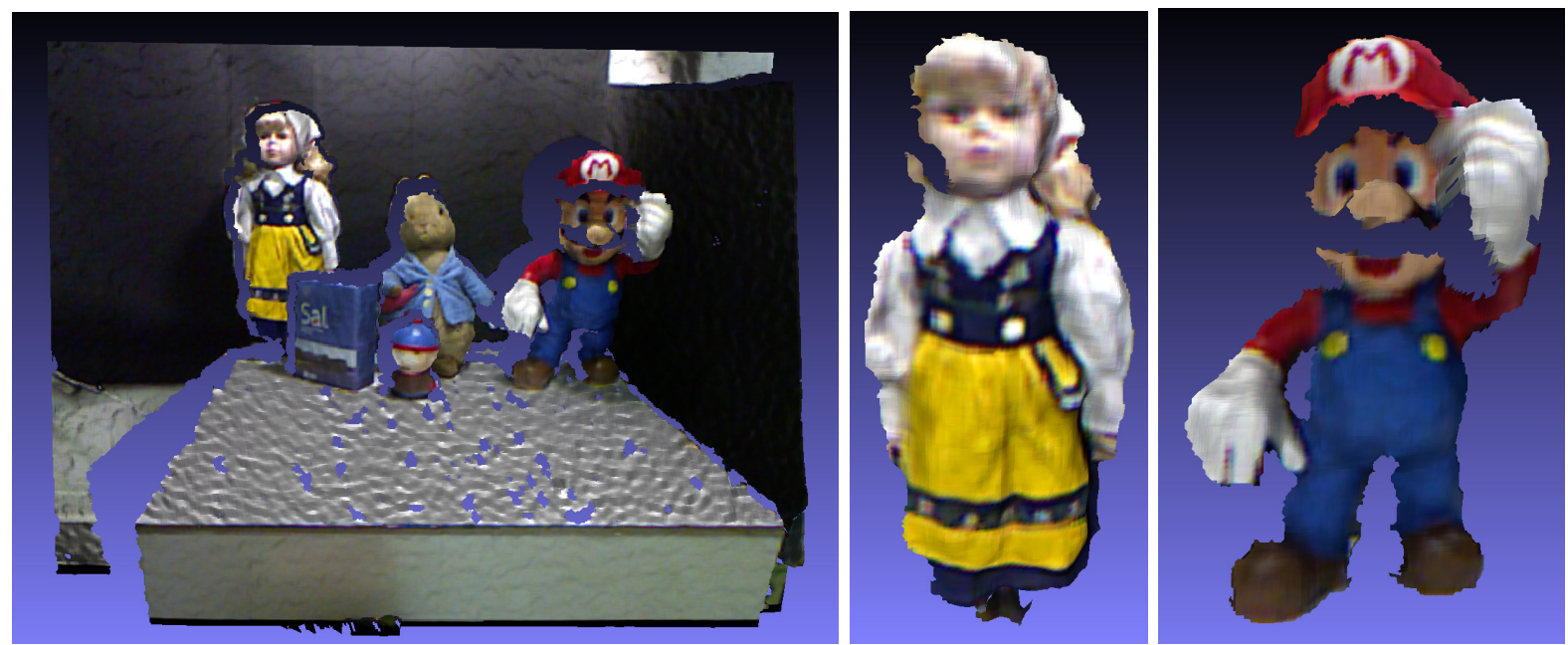

Figure 6.2: A scene named scene005 (left) and two models named dol1018 (middle) and mario000 (right) from the Kinect dataset.

evaluation on the Kinect dataset and compare the proposed B-SHOT descriptor with SHOT, FPFH and RoPS feature descriptors and also with their binary counterparts. From the literature $[64,128]$, SHOT turns out to be the state-of-the-art 3D feature descriptor while the FPFH feature descriptor and RoPS offer competitive performance [64]. All these implementations are publicly available though the Point Cloud Library [126].

\subsubsection{On Selecting B-SHOT over B-RoPS and B-FPFH}

Guo et al. [64] have presented an extensive evaluation of local 3D feature descriptors in various test scenarios and concluded that SHOT [128], RoPS [66] and FPFH [124] feature descriptors offer good performance with some trade-off's in terms of descriptiveness, computational requirements and memory footprint. Hence, the proposed binarization technique was applied to SHOT, RoPS and FPFH feature descriptors to create their binary counterparts, namely, B-SHOT, B-RoPS and B-FPFH. Our experiments with various point clouds have shown that B-SHOT offers a better feature descriptor matching performance compared to B-RoPS and BFPFH, i.e. B-SHOT finds more number of true keypoint correspondences when compared to B-RoPS and B-FPFH. To bolster this fact that B-SHOT offers better performance when compared to B-RoPS and B-FPFH, we employ conventional precision recall curve based evaluation $[128,64]$ and also illustrate an interesting observation from the concept of distance distributions, as mentioned in BRIEF [19], the first binary 2D feature descriptor. Moreover, we also show the number of true correspondences established by B-SHOT, B-RoPS and B-FPFH and show that B-SHOT offers more number of true correspondences. 


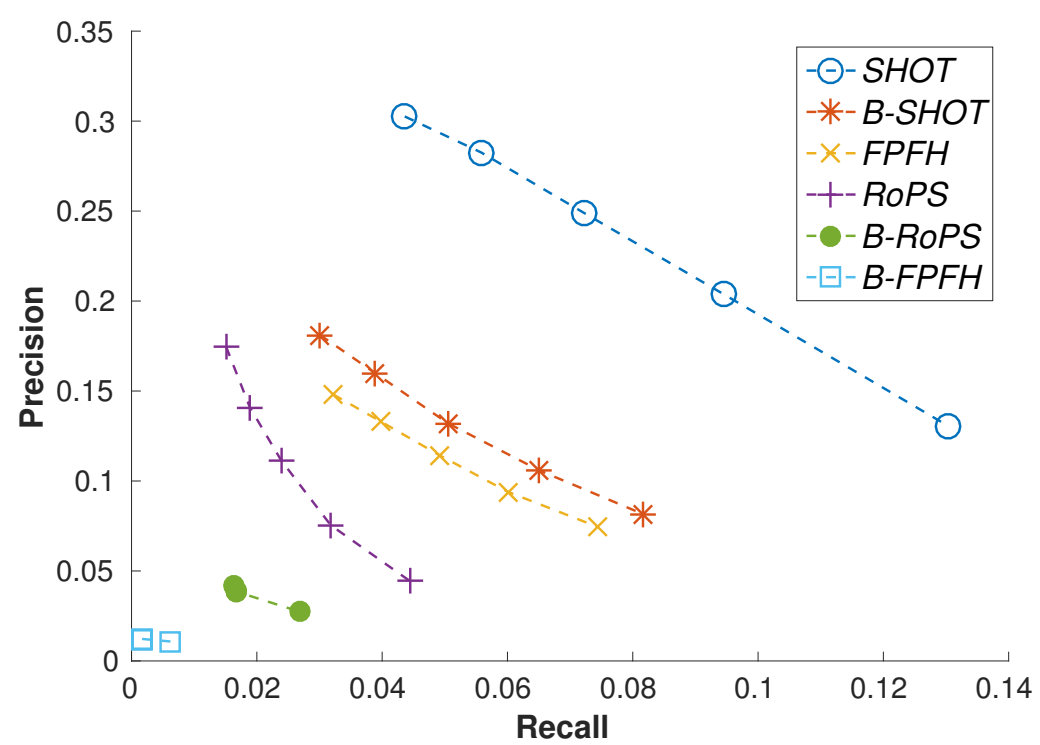

(a) Settings: Uniform keypoints are detected for every $0.01 \mathrm{~m}$ and a support size of $0.12 \mathrm{~m}$ was used to extract feature descriptors.

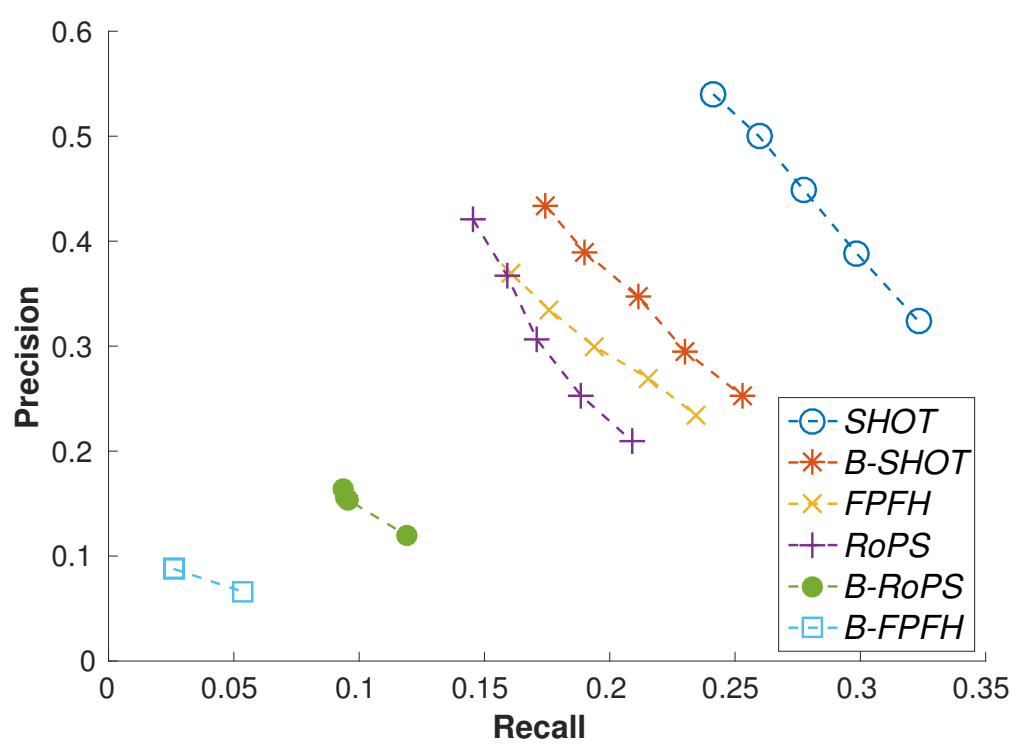

(b) Settings: Uniform keypoints are detected for every $0.02 m$ and a support size of $0.08 m$ was used to extract feature descriptors.

Figure 6.3: Precision Recall curves based evaluation of real valued feature descriptors, SHOT, RoPS, FPFH and their binary counterparts, B-SHOT, B-RoPS and B-FPFH. It can be observed from the above figure that the performance trend is as follows, SHOT $>$ B-SHOT $>$ FPFH $>$ RoPS $>$ B-RoPS $>$ B-FPFH with the employed settings on the Kinect dataset.

Precision-Recall Curve Based Evaluation: Following the evaluation criterion used in state-of-the-art works on 3D feature descriptors, we employ precision-recall curves to compare SHOT, RoPS, FPFH, and their binary versions B-SHOT, B-RoPS and B-FPFH, on the Kinect 
dataset with 49 scene-model pairs. Firstly, we detect uniform keypoints on the model and then based on the available groundtruth 3D transformation, we find scene keypoints that correspond to the previously detected model keypoints. In this way, the 3D feature detector's inaccuracy in finding repeatable keypoints is unaccounted and only the ability of 3D feature descriptor's descriptiveness is evaluated. Then, 3D feature descriptors are calculated at the found model and scene keypoints, and the first and the second nearest scene feature descriptors for every model feature descriptor is found. If the ratio of the first to the second nearest neighbour is less than a threshold $\alpha$, then it is considered as a match or else it is discarded.

To calculate precision and recall, firstly the groundtruth matches $G_{M}$, which represent the number of model keypoints that lie on the scene are calculated. Secondly, the threshold $\alpha$ is varied and the feature descriptor matches, $F D_{\alpha}$ are found based on the above mentioned distance ratio test. Thirdly, the true matches, True $_{\alpha}$, are calculated by finding the number of $F D_{\alpha}$ that comply with the ground truth matches $G_{M}$. Finally, as mentioned by Guo et al. [64], precision and recall at each $\alpha$ is calculated as shown below.

$$
\text { Precision }=\frac{\text { True }_{\alpha}}{F D_{\alpha}} \quad \text { Recall }=\frac{\text { True }_{\alpha}}{G_{M}}
$$

The difference in precision-recall curve experiments presented here compared to the one in Sec. 5.3.2.1 are that, here, first keypoints are detected on the model and then they are transformed on to the scene, while its the opposite in Sec. 5.3.2.1. Here, in the experiments, five values of $\alpha=\{0.9,0.925,0.95,0.975,1\}$ were used for evaluation. The precision and recall values at each value of $\alpha$ is estimated and averaged over 49 scene-model pairs. We performed two experiments by setting the uniform keypoint detection radius $R_{k p}=\{0.01 m, 0.02 m\}$ and the support size $R_{f d}=\{0.12 m, 0.08 m\}$ that is used for calculating feature descriptors. The results of these two experiments are shown in Fig. 6.3 and they highlight that B-SHOT offers second best performance when compared to all others, most importantly, B-SHOT is better slightly better than RoPS and FPFH. The performance trend from Fig. 6.3 can be summarized as SHOT $>$ B-SHOT $>$ FPFH $>$ RoPS $>$ B-RoPS $>$ B-FPFH with the employed settings on the Kinect dataset.

Distance Distributions Based Evaluation: Here we perform the distance distributions experiment as mentioned in BRIEF [19], a 2D binary descriptor. Accordingly, for this experiment, we consider the scene scene005, and the models dol1018 and mario000 from the Kinect dataset, as shown in Fig 6.2. Let us consider scene005 and mario000 as the first case, and scene 005 and dol1018 as the second case. In both cases, we find keypoints with a uniform 

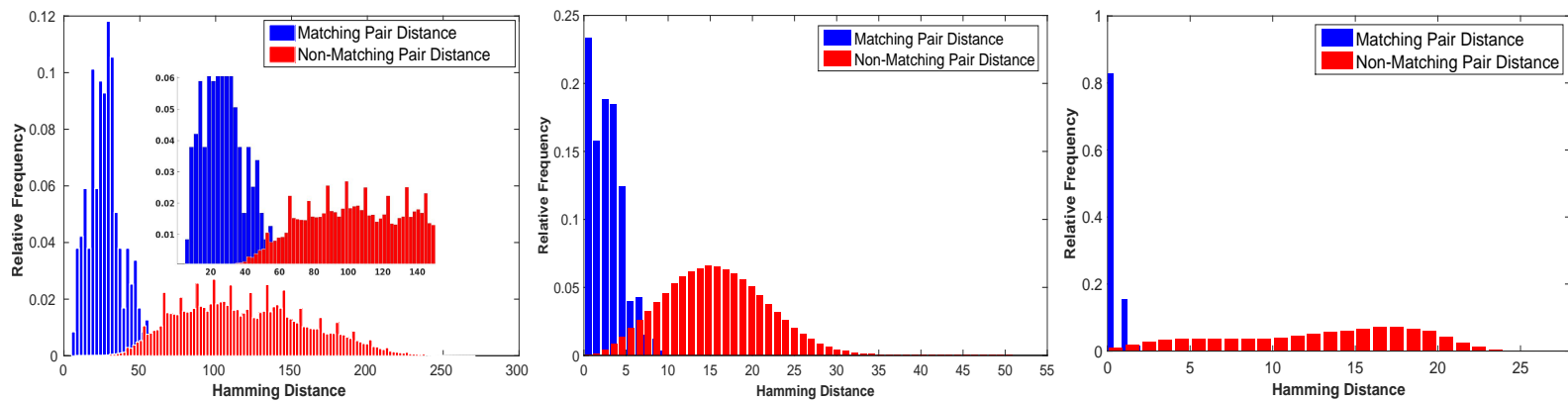

(a) First Case: Distance distributions between scene005 and mario000 of B-SHOT (left), B-RoPS (middle) and B-FPFH (right).
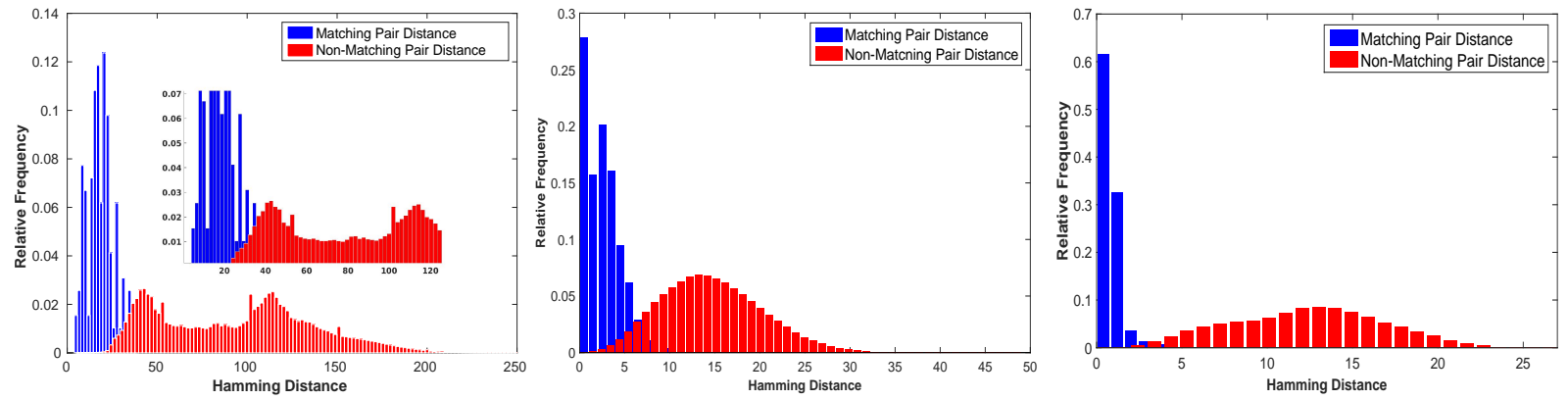

(b) Second Case: Distance distributions between scene0 05 and doll 018 of B-SHOT (left), B-RoPS (middle) and B-FPFH (right).

Figure 6.4: Distance Distributions of B-SHOT, B-RoPS and B-FPFH binary feature descriptors. As can be seen from Fig 6.4(a) and Fig 6.4(b), the left most distance distributions in both cases, which represent B-SHOT, are well separated suggesting the high descriptiveness of B-SHOT when compared to B-RoPS and B-FPFH.

keypoint detector all over the scene and the model with $0.01 \mathrm{~m}$ radius. Then SHOT, FPFH and ROPS descriptors are extracted at all those keypoints with the support radius of $0.12 m$ and their corresponding B-SHOT, B-FPFH and B-RoPS binary feature descriptors are constructed. We match the constructed binary feature descriptors based on the Hamming distance metric and apply RANSAC [53] to remove false correspondences.

Finally, in Fig. 6.4, we show the distribution of Hamming distances between the matching keypoint pairs and the non-matching keypoint pairs in both the test cases with B-SHOT, BFPFH and B-RoPS descriptors. The motivation behind performing the distance distribution experiments is that if the matching pair distance distribution and the non-matching pair distance distribution are well separated, the developed descriptors are highly discriminative [19]. Hence, a simple Hamming distance based classifier can be employed to separate these two classes effectively. 
Table 6.1: Number of true keypoint correspondences found between the scene scene 005 , and models dol1018, and mario000 using B-SHOT, B-RoPS and B-FPFH feature descriptors. A uniform keypoint detector with $R_{k p}=\{0.01 \mathrm{~m}, 0.02 \mathrm{~m}, 0.03 \mathrm{~m}\}$ and feature descriptor support size of $R_{f d}=0.10 \mathrm{~m}$ was employed.

\begin{tabular}{cccc}
\hline \hline scene005 & B-SHOT & B-RoPS & B-FPFH \\
\hline Keypoint Radius: 0.01 $\mathrm{m}$ & & & \\
\hline Doll018 (909 keypoints) & $\mathbf{1 2 8}$ & 74 & 28 \\
Mario000 (860 keypoints) & $\mathbf{2 1 3}$ & 135 & 43 \\
Keypoint Radius: 0.02 $\mathrm{m}$ & & & \\
\hline Doll018 (252 keypoints) & $\mathbf{5 7}$ & 25 & 13 \\
Mario000 (261 keypoints) & $\mathbf{9 4}$ & 53 & 19 \\
Keypoint Radius: 0.03 $\mathrm{m}$ & & & \\
Doll018 (123 keypoints) & $\mathbf{3 3}$ & 17 & 8 \\
Mario000 (125 keypoints) & $\mathbf{4 1}$ & 21 & 15 \\
\hline
\end{tabular}

Findings from Distance Distributions: It can be seen from Fig. 6.4 that in both the cases, as shown in Fig. 6.4(a) and Fig. 6.4(b), B-SHOT's distance distributions are well separated whereas the distance distributions of B-RoPS and B-FPFH are not highly separable. It can be seen from Fig. 6.4(a)(left) that the overlap ends at the Hamming distance of '40' while in Fig. 6.4(b)(left) the overlap ends at a Hamming distance of ' 20 '. This can be seen by zooming the figures. Hence, based on this observation that there is no overlap for Hamming distances less than '20'; a simple Hamming distance based classifier with a threshold of '20' can classify and produce only true correspondences in the case of B-SHOT. In the case of B-RoPS, the threshold for the Hamming distance metric has to be less than ' 2 ' to find true correspondences alone. For, B-FPFH, a simple classifier may not always produce true correspondences irrespective of the threshold in some of the cases, because there is overlap even at a Hamming distance of ' 0 ', as can be seen from Fig. 6.4(a-b)(right).

True Keypoint Correspondences Based Evaluation: Finally, in Table. 6.1, we show the exact number of true keypoint correspondences established by B-SHOT, B-RoPS and BFPFH binary feature descriptors. We consider two test cases as before, i.e., scene 005 and dol1018, and scene005 and mario000. We employ uniform keypoint detector and vary the keypoint radius $R_{k p}=\{0.01 m, 0.02 m, 0.03 m\}$ to detect a varied number of keypoints. As can be seen from Table. 6.1, in both cases with a different number of keypoints, B-SHOT finds a higher number of true correspondences when compared to B-RoPS and B-FPFH. This also holds for other scene-model pairs in the Kinect dataset.

Other Factors for B-SHOT's dominance: It should be noted that there are others factors forming the reasons behind B-SHOT offering good performance, when compared to B-RoPS 
and B-FPFH. Firstly, B-SHOT and B-RoPS are constructed from SHOT and RoPS, which are hybrid feature descriptors [128] that take the advantages of being a combination of signature and histogram based methods, whereas FPFH is just a histogram based method. Secondly, BSHOT contains more information as it has 352 bits, while B-RoPS is 135 bits and B-FPFH is 33 bits in size, hence, making B-SHOT comparatively more descriptive.

Based on these three experiments, the first one based on the precision recall curves, the second one based on distance distributions and the last one based on established true keypoint correspondences, it can be claimed that the proposed binarization technique works better when applied to SHOT rather than RoPS and FPFH.

In Fig. 6.5, we illustrate the B-SHOT correspondences after the RANSAC algorithm between a scene and a model named scene005 and dol1018, from the Kinect dataset, which are shown in Fig. 6.2. In Fig. 6.5, uniform keypoints were detected with radius $R_{k p}=0.01 \mathrm{~m}$ and the employed B-SHOT descriptor support size was $R_{f d}=0.12 m$. As can be seen from the figure, there is no false correspondence after reciprocal nearest neighbour based feature matching and RANSAC. In the figure, the scene and model point clouds are represented in white colour while the keypoints detected on them are shown in blue and red colours respectively.

\subsubsection{Robustifying Recognition Rate for 3D Descriptor Comparison}

Existing works in the 2D image domain $[19,157]$ and 3D point cloud domain $[128,64]$ employ Recognition Rate and Precision vs Recall curves for performance evaluation of feature descriptors. In both these experimental frameworks, they detect keypoints on the model, apply the ground truth $3 \mathrm{D}$ transformation and find the exact same keypoints on the scene. These keypoints are then matched via feature descriptors. In Recognition Rate, the number (or ratio) of correct feature matches are reported. Precision vs Recall curves are generated by varying the threshold that is used to establish the correspondences based on the ratio of the first and the second best nearest neighbours. In Sec. 6.4.2, we presented experimental results using precision-recall curves while selecting B-SHOT over, B-RoPS and B-FPFH. Here, we perform extensive evaluation using a robustified Recognition Rate metric.

Drawbacks of Recognition Rate metric: In the existing Recognition Rate evaluation framework, there are two main drawbacks. Firstly, they do not consider the keypoint detection ambiguity which is significant in 3D domain ${ }^{5}$. Secondly, they apply the available groundtruth 3D transformation on the model keypoints to find the exactly same keypoints on the scene,

\footnotetext{
${ }^{5}$ State-of-the-art 3D keypoint detectors achieve at most 0.5 relative repeatability [147], i.e., only half of the detected keypoints between a scene and a model lie exactly at the same positions.
} 


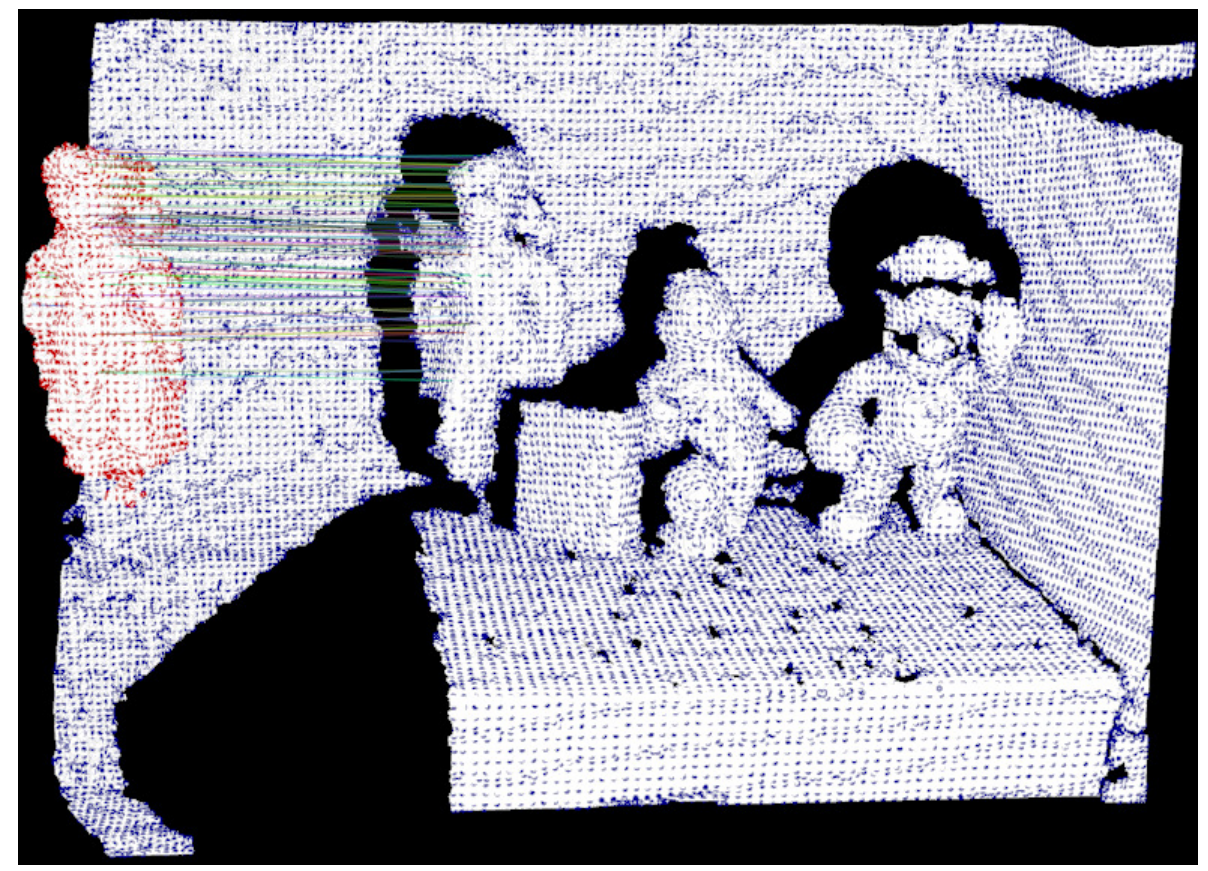

(a) Front View

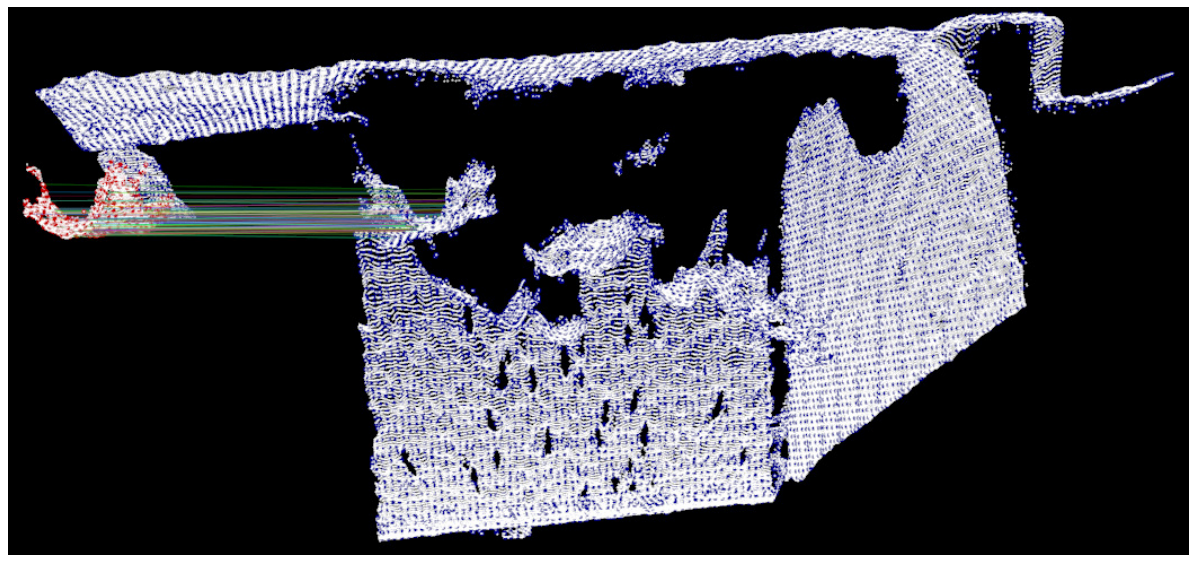

(b) Top View

Figure 6.5: B-SHOT correspondences after RANSAC on a scene scene005 and model dol1 018 from the Kinect dataset as shown in Fig. 6.2. It can be seen that there is no false correspondence. Scene and model point clouds are represented in white colour while the keypoints detected on them in blue and red colours respectively.

hence they do not consider the false positives that may arise from the back ground of the scene in which models are present. Therefore we propose an evaluation metric, Robust Recognition Rate that addresses the above-mentioned issues.

The two main important traits that are expected from a good 3D feature descriptor:

1. Ability to match the keypoints that arise from similar areas with small position ambiguity due to the imperfection of current 3D keypoint detectors. 
2. Avoiding false positives that arise from back ground and from other models that are present in the scene.

Hence, we propose to employ a uniform keypoint detector to detect keypoints with the highest ambiguity in keypoint repeatability (to account for the worst case scenario). The uncertainty in the keypoint detection can be varied accordingly with the keypoint detection radius $R_{k p}$. Next, this uniform keypoint detector is used to find keypoints all over the scene and the model to allow for all the possible false correspondences from the back ground and other models present in the scene. Our experiments with SHOT, FPFH and RoPS have shown that even these real valued state-of-the-art 3D feature descriptors generate some false correspondences in almost all of the scenarios. It is therefore inevitable to use RANSAC [53] based 3D transformation estimation, to remove those false correspondences. Moreover, the distance ratio based nearest neighbour matching, in general, is not employed in any real world application, instead the first nearest neighbour is assigned as a match and RANSAC is later employed to prune outliers. Hence we propose a robust evaluation metric.

\section{Steps to estimate Robust Recognition Rate (RRR):}

1. Detect uniform 3D keypoints with a voxel grid filter of radius $R_{k p}$ on both the scene and the model independently. This caters for the possible ambiguity from 3D keypoint detection, which can be varied accordingly with $R_{k p}$ and considers all the possible false correspondences from back ground of the scene as well.

2. Extract 3D feature descriptors with a support radius of $R_{f d}$ around the detected keypoints and establish nearest neighbour correspondences that are reciprocal.

3. Apply RANSAC to find the final set of correspondences and estimate the 3D transformation $T_{R}$ using the maximal consensus set.

$T_{d i f f}$ Error Metric or Frobenius Norm: We then compute the difference, $T_{\text {diff }}$, between the estimated 3D transformation via RANSAC, $T_{R}$, and the available ground truth transformation, $T_{G T}$, by employing the Euclidean metric as shown below:

$$
T_{\text {diff }}=\sqrt{\sum_{i=0}^{n} \sum_{j=0}^{n}\left(T_{R_{i j}}-T_{G T_{i j}}\right)^{2}}
$$

where $n=3$ in the case of $3 \mathrm{D}$ homogeneous transformation matrices, and $T_{R_{i j}}$ and $T_{G T_{i j}}$ represent the corresponding elements at indices $\{i, j\}$ in the transformation matrices. The $T_{d i f f}$ 
error metric provides a quantitative measure of how well the established correspondences fulfil the purpose of accurately estimating the 3D transformation between the scene and the model. A greater value or a peak in $T_{\text {diff }}$ implies that the estimated 3D transformation is considerably different from the groundtruth highlighting the possibility of no true keypoint correspondences.

Once the 3D transformation is estimated, the next step is to evaluate how many detected keypoints are positively matched via feature descriptors. To quantify this, we find the number of true RANSAC correspondences by verifying their compliance with the available groundtruth transformation.

4. We transform the detected scene keypoints onto the model by applying the groundtruth transformation and check if the established RANSAC keypoint correspondences lie within a neighbourhood of $\epsilon$. If a RANSAC keypoint correspondence lies within this $\epsilon$, it is considered to be a true RANSAC match.

We provide the ratio of true RANSAC matches to the number of detected keypoints that are present in both the scene and the model, and term it as Robust Recognition Rate (RRR). To find the number of keypoints present in both the scene and the model, we use the same method as employed in repeatability tests [147], wherein, we transform the model keypoints onto the scene and verify if there is any keypoint present in the scene within a neighbourhood of $\epsilon$. If yes, it is considered to be a keypoint that is present in both the scene and the model.

Robust Recognition Rate ( $R R R$ ) metric: $R R R$ is an enhancement to the Recognition Rate that was employed in $[19,20]$. In short, there are three enhancements, firstly, in $R R R$, the ambiguity in 3D keypoint detection is considered by employing a uniform keypoint detector. Secondly, the feature descriptor's capability in avoiding all possible false correspondences is evaluated as the keypoints are detected on both the model and scene independently without applying the groundtruth transformation. Lastly, a more realistic setting is added where the first nearest neighbours are assigned as the matches and RANSAC is employed to prune outliers. Then, Robust Recognition Rate $(R R R)$ is estimated as shown below.

$$
R R R=\frac{\text { True matches in RANSAC correspondences } \times 100}{\text { Number of keypoints in both model and scene }}
$$

\section{$T_{d i f f}$ and $R R R$ metrics for descriptor's performance evaluation:}

The $T_{\text {diff }}$ and $R R R$ metrics complement each other in providing substantial information about the feature descriptor's performance. A low value in the $T_{\text {diff }}$ metric at a specific scene-model pair index highlights that the estimated transformation is close to the groundtruth while the 
corresponding $R R R$ metric at the same scene-model pair index provides a quantitative measure of the established true correspondences. A peak in the $T_{\text {diff }}$ metric highlights a potential failure case and the corresponding $R R R$ metric goes to zero, as there will be no true correspondences. In general, we found that a $T_{\text {diff }}$ value greater than 1.5 or 2 is a failure case where the feature descriptors cannot establish any true feature correspondences and correspondingly the $R R R$ metric goes to zero. It is easy to infer a successful case by looking solely at the $R R R$ metric, as it would have a high $R R R$ value. But, when the $R R R$ value is close to zero, then the $T_{\text {diff }}$ metric turns out to be helpful in concluding if the estimated transformation is correct or not.

\subsubsection{Comparison of B-SHOT with SHOT, RoPS and FPFH}

In this section, we evaluate and compare B-SHOT with other real valued feature descriptors based on $T_{\text {diff }}$ and $R R R$ metrics. $T_{\text {diff }}$ evaluates if the found correspondences successfully estimate the 3D transformation between the scene and the model, whereas $R R R$ provides the ratio of correspondences found between the scene and the model. Through these experiments, it can observed that there is a small drop in the number of correspondences found using B-SHOT when compared to SHOT, however, the found correspondences are good enough to estimate the $3 \mathrm{D}$ transformation, which is the main application of $3 \mathrm{D}$ feature descriptors.

We perform two experiments on the Kinect dataset with different parameter settings for keypoint detection and feature description. In Experiment 1, the keypoints are close to each other and the neighbouring feature descriptors have large overlap, while in Experiment 2, keypoints are farther away with higher position ambiguity when compared to feature descriptor's support size.

Experiment 1: In Fig. 6.6, we compare B-SHOT with the state-of-the-art real valued feature descriptors, SHOT, RoPS and FPFH, based on the $T_{\text {diff }}$ (Eqn. 6.1) and $R R R$ (Eqn. 6.2) metrics on the Kinect dataset. The keypoints were detected using a voxel grid filter (uniform keypoint detector) with leaf size, $R_{k p}=0.01 \mathrm{~m}$ and feature descriptors were extracted with support size, $R_{f d}=0.12 \mathrm{~m}$. Reciprocal nearest neighbour correspondences between scene and model keypoints are established by feature descriptor matching. The Euclidean distance metric is employed for real valued feature descriptors, SHOT, FPFH and RoPS and the Hamming distance metric is used for the B-SHOT. Finally, the best consensual set of matches are found using the RANSAC algorithm and the $T_{\text {diff }}$ and $R R R$ metrics are estimated. We perform these steps on all 49 scene-model pairs in the Kinect dataset. In Fig. 6.6, the employed parameter values of $R_{k p}=0.01 \mathrm{~m}$ for uniform keypoint detector, and $R_{f d}=0.12 \mathrm{~m}$ for descriptor support radius, highlight the scenario where the detected keypoints are close to each 


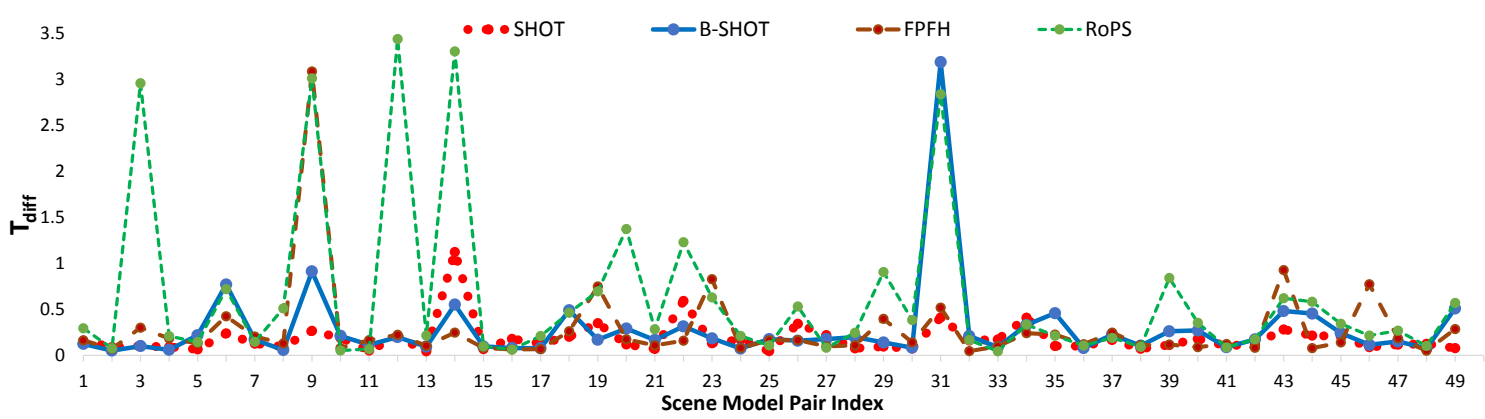

(a) Comparison of B-SHOT with SHOT, RoPS and FPFH based on $T_{\text {diff }}$ metric on the Kinect dataset.

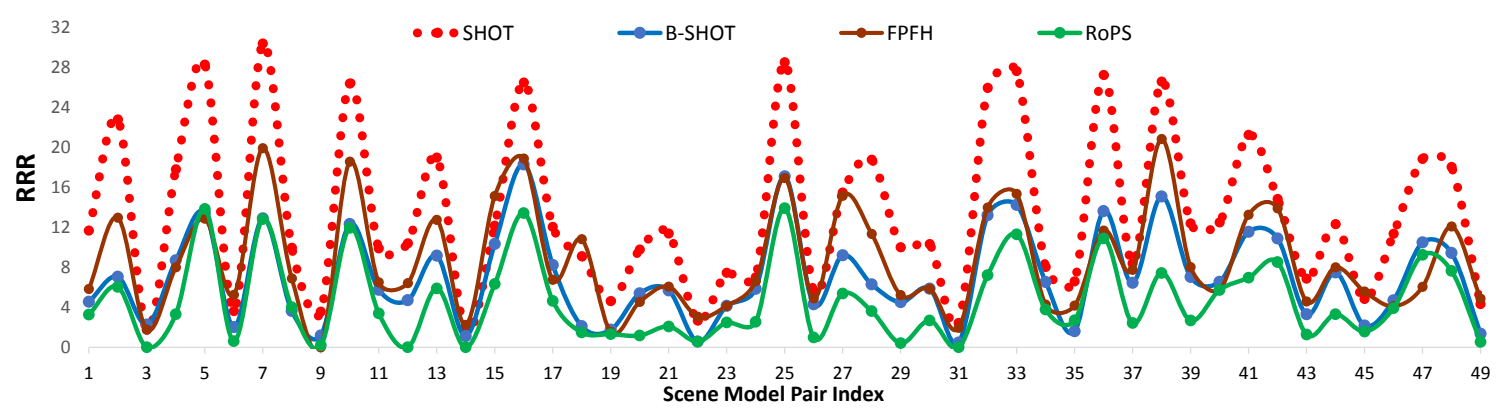

(b) Comparison of B-SHOT with SHOT, RoPS and FPFH based on $R R R$ metric on the Kinect dataset.

Figure 6.6: Comparison of B-SHOT with SHOT, RoPS and FPFH based on the $T_{\text {diff } f}$ and $R R R$ metrics. Parameters: $R_{k p}=0.01 \mathrm{~m}$ and $R_{f d}=0.12 \mathrm{~m}$. These values of $R_{k p}=0.01 \mathrm{~m}$ and $R_{f d}=0.12 m$ highlight the possibility where the detected keypoints are very close and there is large overlap and interference between the neighbourhood keypoint descriptors. A peak in the $T_{\text {diff }}$ metric highlights a failure case where there is a significant error between the estimated $3 \mathrm{D}$ transformation and the available groundtruth. A value close to zero in $R R R$ metric highlights that the number of true RANSAC correspondences are much lesser when compared to the detected keypoints.

other, have less position ambiguity, while having large overlap and interference between the neighbouring keypoint descriptors.

Observations: It can be seen from the $T_{\text {diff }}$ metric, as shown in Fig. 6.6(a), that RoPS fails in more cases when compared to others. It can be simultaneously observed from the $R R R$ metric, (Fig. 6.6(b)), that at those corresponding indices where $T_{\text {diff }}$ has peaks (for example, index $=\{3,12\})$, the $R R R$ value of RoPS goes to zero, indicating that there are no true correspondences. B-SHOT fails only in one case (index = 31) out of the 49 scenemodel pairs. Though B-SHOT has slightly higher $T_{\text {diff }}$ values at indices 6 and 9, they are not failure cases as the corresponding $R R R$ values are not exactly zero, highlighting the presence of few true correspondences.

From Fig. 6.6(b), it can be seen that RoPS offers lower true RANSAC correspondences 
ratio when compared to B-SHOT, SHOT and FPFH descriptors on the Kinect dataset with these parameter settings. B-SHOT offers a lower ratio of true correspondences when compared to SHOT and FPFH because of the previously mentioned characteristics of being a binary 3D feature descriptor. If $R R R$ metric alone is seen (Fig. 6.6(b)), it may give an impression that B-SHOT offers a lower number of true RANSAC correspondences ratio, but through the $T_{\text {diff }}$ metric (Fig. 6.6(a)) it can be ensured that this decrease in feature correspondences does not affect the main purpose of $3 \mathrm{D}$ transformation estimation via feature descriptor matching. Once a rough 3D transformation is estimated, ICP algorithm [13] can be used to perform fine and accurate registration, which inherently provides more number of correspondences if required.

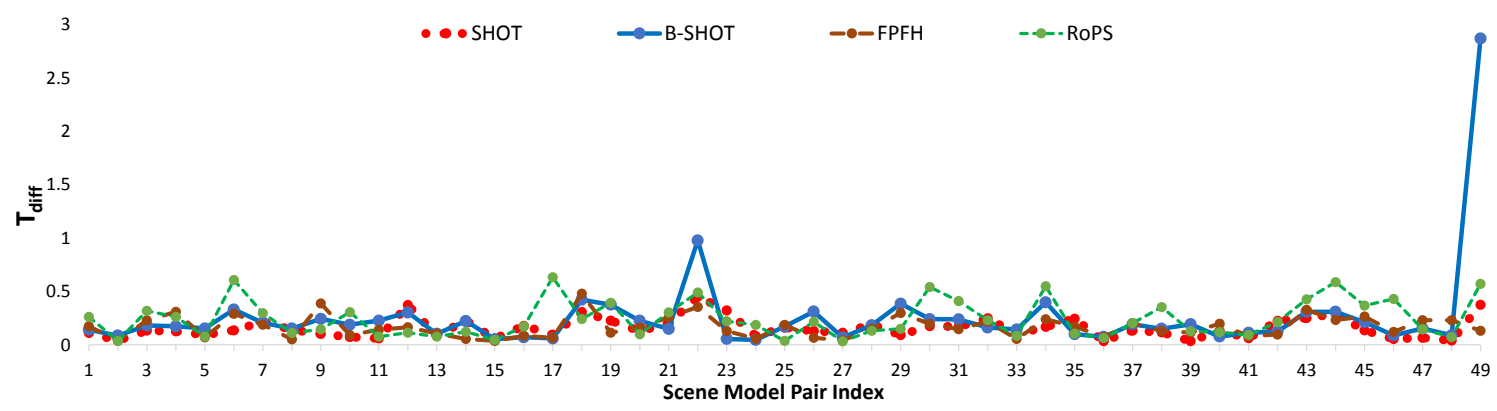

(a) Comparison of B-SHOT with SHOT, RoPS and FPFH based on $T_{\text {diff }}$ metric on the Kinect dataset.

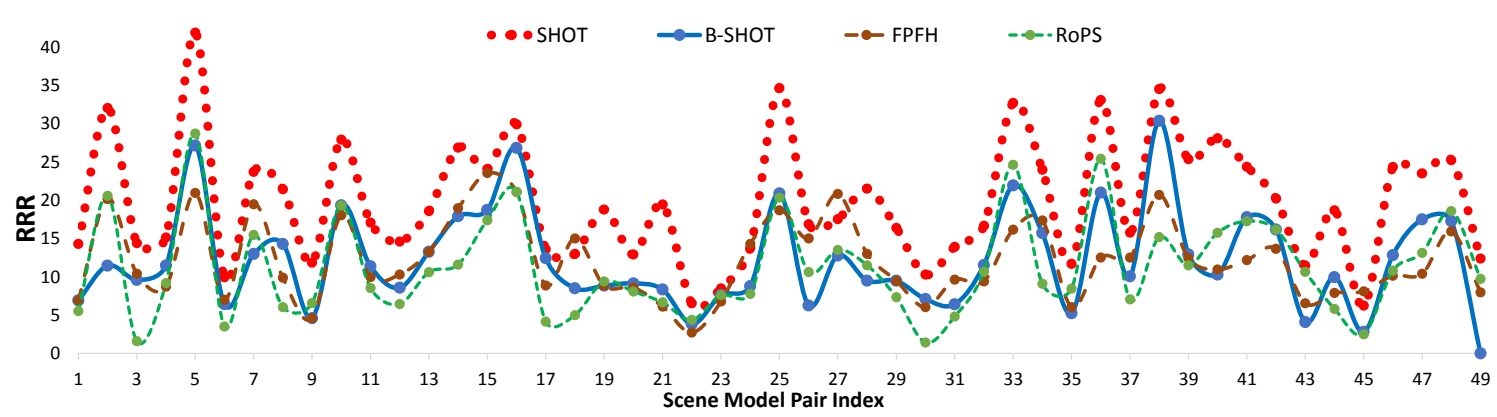

(b) Comparison of B-SHOT with SHOT, RoPS and FPFH based on $R R R$ metric on the Kinect dataset.

Figure 6.7: Comparison of B-SHOT with SHOT, RoPS and FPFH based on the $T_{\text {diff }}$ and $R R R$ metrics. Parameters: $R_{k p}=0.02 \mathrm{~m}$ and $R_{f d}=0.08 \mathrm{~m}$. These values of $R_{k p}=0.02 \mathrm{~m}$ and $R_{f d}=0.08 \mathrm{~m}$ highlight the possibility where the detected keypoints are bit further when compared to the feature descriptor's support size. It can be seen that B-SHOT fails only in one case (index value $=49$ ) while performing well on all other scene-model pairs.

Experiment 2: In this experiment, keypoints were detected with $R_{k p}=0.02 \mathrm{~m}$ and feature descriptors were extracted with support size, $R_{f d}=0.08 \mathrm{~m}$. This resembles a scenario where the detected keypoints $\left(R_{k p}=0.02 m\right)$ are farther when compared to the employed descriptor's support size $\left(R_{f d}=0.08 m\right)$, while having greater position ambiguity in keypoint detection and 
Table 6.2: Robust Recognition Rate $(R R R)$ as offered by various feature descriptors in experiments $1 \& 2$ on the Kinect dataset.

\begin{tabular}{ccc}
\hline \hline Descriptor & $R R R_{\operatorname{Exp~} 1}$ & $R R R_{\operatorname{Exp} 2}$ \\
\hline SHOT & 13.43 & 19.78 \\
FPFH & 8.6 & 12.20 \\
B-SHOT & 7.03 & 12.20 \\
RoPS & 4.59 & 11.16 \\
\hline
\end{tabular}

lesser overlap in neighbouring feature descriptors. We perform the same steps of finding true RANSAC matches and the consensual transformation to estimate $T_{\text {diff }}$ and $R R R$ metrics for all the scene-model pairs in the Kinect dataset.

Observations: It can be seen from the $T_{\text {diff }}$ metric, as shown in Fig. 6.7(a), that B-SHOT fails in one case (index $=49$ ) while working well in all other cases. Fig. 6.7(b) shows that B-SHOT's $R R R$ value goes to zero at index $=49$. The reason behind this is that the created BSHOT descriptors were not discriminative enough to find true correspondences in this specific scene-model pair. Except the one failure case, B-SHOT's performance based on the $R R R$ metric is comparable to RoPS in this scenario. In comparison with the previous settings, it can be seen that the $R R R$ value of B-SHOT and other feature descriptors is higher because there is less interference in the neighbourhood keypoint descriptors.

FPFH offered good feature matching performance on the Kinect dataset but its computational time increases exponentially with the increase in the support radius ${ }^{6}$. Guo et al. [64], through their extensive performance evaluation, highlighted that FPFH is preferable when the points in the local neighbourhood are relatively less. Therefore FPFH is not preferable in terms of computational requirements when the point clouds are dense and the support size for the feature descriptor is relatively bigger. It turns out that in our experiments, B-SHOT offers better performance than the RoPS feature descriptor on the Kinect dataset with the employed settings.

In Table. 6.2, we present the averaged $R R R$ values offered by SHOT, FPFH, B-SHOT and RoPS descriptors on the Kinect dataset in Experiments $1 \& 2$. Please note that in both these experiments the normal estimation radius is set to $0.02 \mathrm{~m}$ while the $R A N S A C_{\text {inlier }}$ threhsold is set to 0.01. It can be seen from Table. 6.2 that SHOT established more number of true correspondences, followed by FPFH, B-SHOT and the RoPS. 


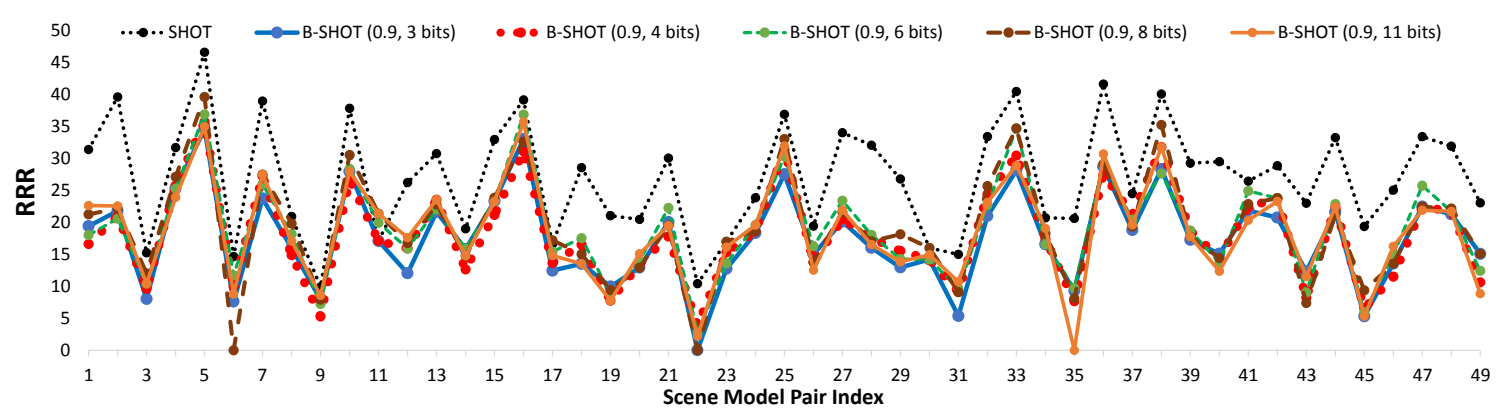

(a) B-SHOT's performance with the change in the number of encoded real values, $m=\{3,4,6,8,11\}$, on the Kinect dataset. Used Parameters: $R_{k p}=0.02 m$ and $R_{f d}=0.10 m$. The estimated mean standard deviation is 1.64 .

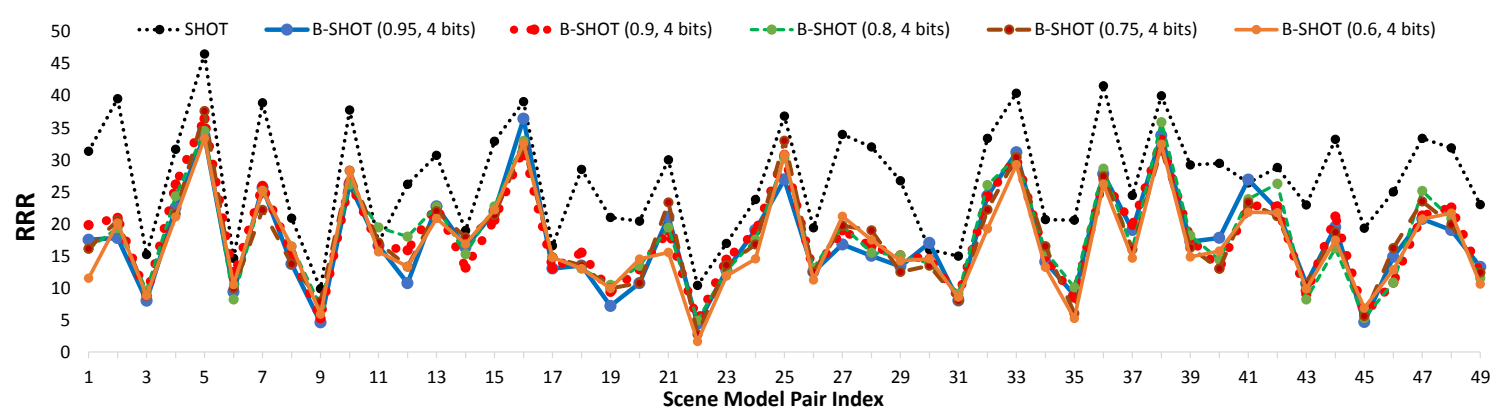

(b) B-SHOT's performance with the change in encoding ratio, $E_{r}=\{0.95,0.9,0.8,0.75,0.6\}$, on the Kinect dataset. Used Parameters: $R_{k p}=0.02 \mathrm{~m}$ and $R_{f d}=0.10 \mathrm{~m}$. The estimated mean standard deviation is 1.53 .

Figure 6.8: Performance of B-SHOT with the change in free parameters, $m$ (number of encoded real values) and $E_{r}$ (encoding ratio) evaluated using $R R R$ metric on the Kinect dataset.

\subsection{Parameter Evaluation}

The proposed binarization technique in Sec. 6.3 that is used to convert a real valued feature descriptor to a binary feature descriptor has two free parameters. Firstly, $m$, the number of real values that are encoded into binary values. Secondly, the encoding ratio, $E_{r}$, which is employed to quantize the real values to create binary representation. In all the experiments performed in the previous section, $m$ was set to 4 and the encoding ratio, $E_{r}$ was set to 0.9 . In this section, we vary these two parameters, $m$ and $E_{r}$, and show the performance of B-SHOT. We use the $R R R$ metric, which represents the ratio of true RANSAC matches to the actual keypoints to evaluate the performance of B-SHOT with the change in the parameters, $m$ and $E_{r}$. For these parameter evaluation experiments, we use the Kinect dataset, extract keypoints with $R_{k p}=0.02 \mathrm{~m}$ and feature descriptors with $R_{f d}=0.10 \mathrm{~m}$ while the false correspondences are removed with RANSAC.

\footnotetext{
${ }^{6}$ This can also be seen from Fig. 9 of Salti et al. [128]
} 
Performance of B-SHOT with variation in $m$ parameter: In Fig. 6.8(a), we show the performance of B-SHOT while varying the parameter $m=\{3,4,6,8,11\}$ and keeping $E_{r}=$ 0.9 as a constant. For reference, we also show the performance of SHOT feature descriptor with the same settings of $R_{k p}=0.02 m$ and $R_{f d}=0.10 m$ in the figure. It can be seen from Fig. 6.8(a) that there is no significant variation but a minimal change (std. dev $=1.64$ ) in B-SHOT's performance with the change in the parameter $m$. In particular, there is a drop in the performance, in the cases where $m=8$ and $m=11$ at index values of 6 and 35, correspondingly. There is a slight drop in the performance at index values of 12 and 31, when $m=3$. In general, $m=\{4,6\}$ offered similar performance and can be considered as a good choice for the proposed binarization technique to create B-SHOT from SHOT.

Performance of B-SHOT with variation in $E_{r}$ parameter: In Fig. 6.8(b), we show the performance of B-SHOT while varying the values of $E_{r}=\{0.95,0.9,0.8,0.75,0.6\}$ and keeping $m=4$ as a constant. The values of $R_{k p}$ and $R_{f d}$ are exactly the same as above where we change the parameter $m$. It can be seen from Fig. 6.8(b) that there is no significant variation ( $\operatorname{std} . \mathrm{dev}=1.53$ ) in the performance of B-SHOT with the change in the $E_{r}$ parameter, except for small fluctuations when $E_{r}=0.95$ and $E_{r}=0.6$ at the index values of 19 and 22, correspondingly.

From this extensive performance evaluation based on $T_{\text {diff }}$ and $R R R$ metrics, evaluating the performance of B-SHOT with the change in free parameters, $m$ and $E_{r}$ and considering both the success and failure scenarios, it can be claimed that B-SHOT, being a binary descriptor, offers a competitive keypoint matching performance to the state-of-the-art real valued 3D feature descriptors. However, B-SHOT's advantages of having a very low memory footprint with faster descriptor matching capabilities outweigh its loss in descriptiveness.

\subsection{Computational \& Memory Requirements}

One of the biggest advantages of a binary feature descriptor is that feature descriptor matching can be performed extremely quickly. B-SHOT feature descriptors are matched using the hamming distance metric. The hamming distance between two binary vectors can be computed as the bitcount of the vector that results from the XOR operation of those two input binary vectors. This can be computed even faster on CPU's that have SSE 4.1 enabled, which supports the POPCNT instruction. 
Table 6.3: Computational time (in seconds) required for matching B-SHOT and SHOT feature descriptors

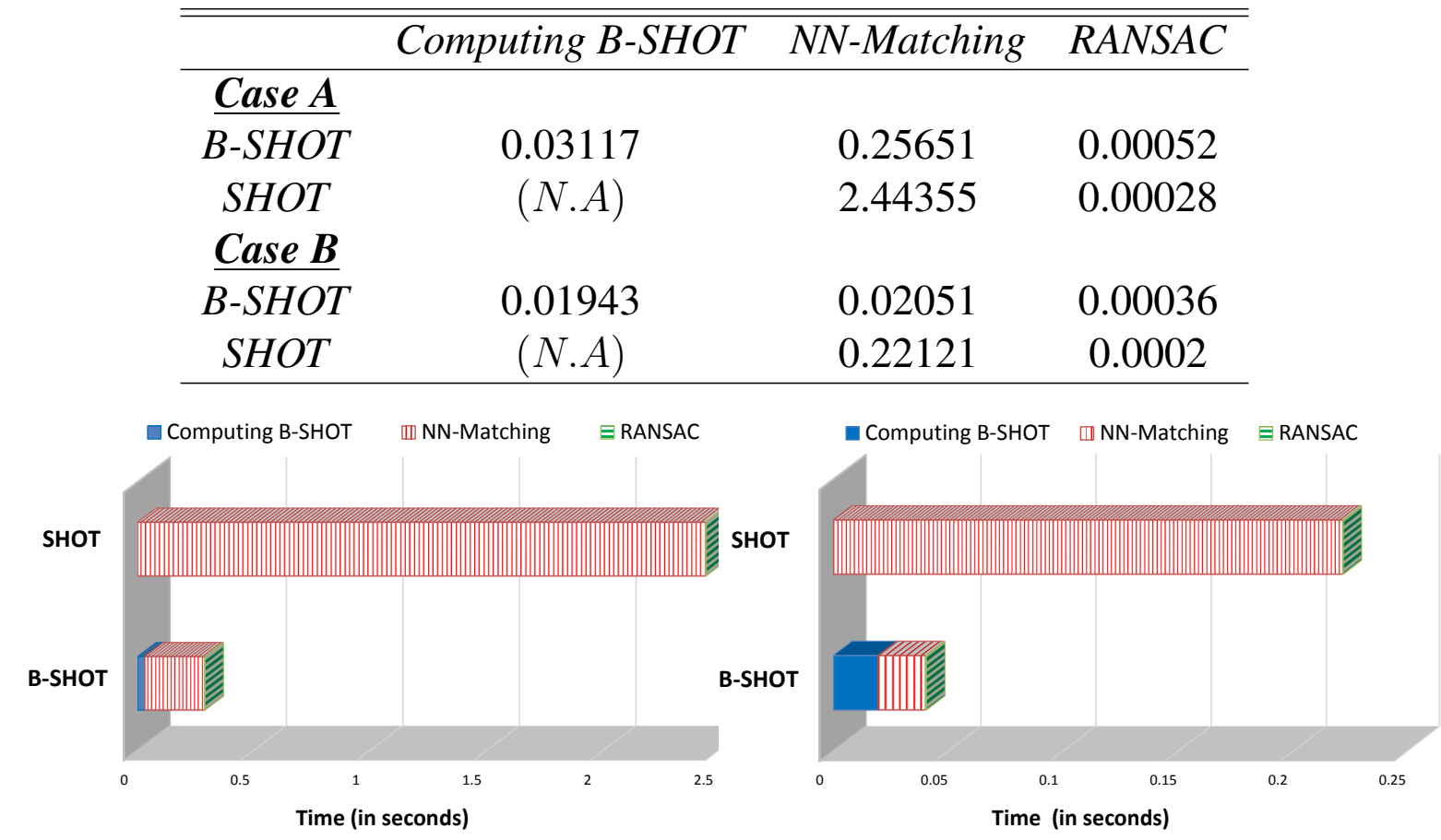

(a) Case A: $\left\{R_{k p}=0.01 m\right.$ and $\left.R_{f d}=0.12 m\right\}$. (b) Case B: $\left\{R_{k p}=0.02 m\right.$ and $\left.R_{f d}=0.08 m\right\}$.

Figure 6.9: Computational time (in seconds) for B-SHOT and SHOT feature descriptor based matching of keypoints with different parameters on the Kinect dataset.

\subsubsection{Comparing the Computational Requirements of B-SHOT \& SHOT}

Experimental Settings: We compare the computational time required for SHOT and B-SHOT descriptor based matching of keypoints in Fig. 6.9 and Table. 6.3. As the B-SHOT descriptor is created from the SHOT descriptor, the extra time required to create B-SHOT from SHOT is also considered in these experiments as shown in Fig. 6.9. Similar to previous experiments as performed in Fig. 6.6 and Fig. 6.7, two cases with exactly same parameter settings on the Kinect dataset are considered for computational time evaluation. In Case A, as shown in Fig. 6.9(a), $R_{k p}$ was set to $0.01 \mathrm{~m}$ and $R_{f d}$ was set to $0.12 \mathrm{~m}$. In the Case $B, R_{k p}=0.02 \mathrm{~m}$ and $R_{f d}=0.08 \mathrm{~m}$ and the computational time is shown in Fig. 6.9(b). The number of detected keypoints is much lower in Case $B$ when compared to Case $A$ because of the value of $R_{k p}$. The time taken for computing B-SHOT from SHOT, matching B-SHOT descriptors between the model and the scene keypoints, and finding the final correspondences via RANSAC are all computed and averaged over the 49 samples in the Kinect dataset. Please note that the nearest neighbours for SHOT feature descriptor matching were obtained by creating a kdtree 
representation ${ }^{7}$ and retrieving only the reciprocal correspondences.

Observations: It can be observed from Fig. 6.9 that the time taken for B-SHOT computation and matching is much lesser than the SHOT feature descriptor based NN-matching alone. Table. 6.3 shows the exact values of the computational time for the same. Case A refers to that in Fig. 6.9(a) while Case B refers to that in Fig. 6.9(b). In Case A, B-SHOT feature descriptor computation and matching with RANSAC is about 8.4 times faster than SHOT descriptor matching, whereas in Case B, B-SHOT is 5.2 times faster than SHOT descriptor matching. This is because, in Case A, there are a greater number of keypoints and hence SHOT descriptor matching was even more computationally intensive when compared to Case B. From these experiments, we can conclude that B-SHOT feature descriptor matching is around 6 times faster than SHOT descriptor matching, thereby having immense potential in applications such as online 3D object recognition $[63,47]$ and large scale 3D point cloud retrieval applications [28].

B-SHOT has lower descriptor matching complexity but not extraction: In general there are two steps for 3D keypoint matching via feature descriptors, the first being feature descriptor extraction while the second is feature descriptor matching. B-SHOT offers significant boost in feature descriptor matching stage as it can be matched using Hamming distance metric. An important point to note is that, as both B-SHOT and SHOT feature descriptors have the same offset computation time for extracting SHOT feature descriptors, which is not shown in the figure and the table as it would overshadow the enhancement offered by B-SHOT in feature descriptor matching stage. The actual computation time required for extracting SHOT feature descriptors on a single scene model pair in Case A and Case B is $50.1 \mathrm{sec}$ and $16.42 \mathrm{sec}$ respectively, while running on a single thread.

Multithreaded and GPU implementations for faster extraction: The SHOT implementation as available in Point Cloud Library [126] comes with a multi-threaded implementation. On the employed CPU for experimentation which had 12 CPU cores, the SHOT feature descriptor extraction required $10.4 \mathrm{sec}$ and $3.5 \mathrm{sec}$ of wall clock time for Case $A$ and Case B respectively. This SHOT feature descriptor extraction time also varies with the number of keypoints, i.e., in Case A, keypoints are extracted for every $0.01 \mathrm{~m}$ while in CaseB, they were extracted at $0.02 \mathrm{~m}$ and hence is the difference in extraction time. Moreover, the SHOT feature descriptor ported on GPU [105, 75], can offer 40x-54x speed up in feature descriptor extraction and hence feature descriptor extraction is not a bottleneck with available multi-core CPUs

\footnotetext{
${ }^{7} \mathrm{We}$ employ pcl::registration::CorrespondenceEstimation class from Point Cloud Library (www.pointclouds.org) to estimate reciprocal correspondences, which inherently uses a kdtree for faster matching and retrieval.
} 
and GPUs. More importantly, in the applications such as large scale retrieval or 3D mobile visual search where feature descriptors are extracted only once but feature descriptor matching is carried out multiple times with a database of point clouds, faster descriptor matching and low memory footprint as offered by B-SHOT turn out to be more advantageous.

Memory Requirements: As mentioned earlier, binary descriptors have an edge over conventional feature descriptors in the memory required to store and represent them. While SHOT requires 1408 bytes (as a float value requires 4 bytes according to IEEE 754 single-precision binary floating-point format), as it is a 352 dimensional vector with each value ranging from 0 to $1, \mathrm{~B}-\mathrm{SHOT}$ requires only 352 bits of binary data for its representation. There is a $32-$ fold reduction in the memory required to represent and store B-SHOT feature descriptors when compared to SHOT feature descriptors. This can be helpful in applications that require online transfer of feature descriptors as binary feature descriptors require much lesser bandwidth.

\subsection{Conclusion}

In this chapter, we have introduced the very first binary 3D feature descriptor, B-SHOT, for fast keypoint matching on 3D point clouds. Specifically, a binarization method is proposed to convert a real valued feature descriptor to a binary feature descriptor. We applied the proposed binarization onto three state-of-the-art real valued 3D feature descriptors and showed qualitatively and quantitatively that it offers best performance when applied to the SHOT [128] feature descriptor. Later, we robustified existing Recognition Rate metric for 3D feature descriptor evaluation, and called, Robust Recognition Rate $(R R R)$, by considering all possible false feature correspondences and catering for the ambiguity in 3D keypoint detection. Our experiments showed that the proposed B-SHOT offers comparable keypoint matching performance while having 32-fold less memory footprint and is approximately 6 times faster in feature descriptor matching. This work highlights that binary 3D feature descriptors are feasible and opens up a research direction for creating highly efficient binary 3D feature descriptors.

The proposed B-SHOT in this chapter requires 352 bits and offers reduced keypoint matching performance than original SHOT descriptor. In the next chapter, we explore if we can further reduce the number of bits required for a single SHOT descriptor while improving the performance. It is shown that this is possible through lattice quantization and we present a spectrum of possible bitrates and achievable performance of the created low bitrate 3D descriptors. 


\section{Chapter 7}

\section{Low Bit-rate 3D Feature Descriptors with Lattice Quantization}

\subsection{Introduction}

\section{Application: Online transfer of 3D feature descriptors}

Let us consider an application scenario, wherein a 3D point cloud captured with a hand-held mobile device has to be matched with a database of 3D point clouds present on a remote server. This is relevant in applications, in which a user, or an autonomous robot deployed in an unknown environment, needs to perform tasks such as 3D object recognition [47], retrieval [129], reconstruction [113], 3D point cloud search and registration [65, 112, 64], Simultaneous Localization and Mapping (SLAM) $[43,111]$ and loop closure, while interacting with a remote server. One of the most important steps in all the above mentioned applications, is to establish reliable 3D keypoint correspondences between a source and a target point cloud. 3D feature descriptors are the preferred choice to find reliable 3D keypoint correspondences between two arbitrarily oriented source and target point clouds.

It was observed in 2D image domain that compressing and transmitting 2D feature descriptors requires less bandwidth, has lower power consumption and offers better user experience than transmitting a downsampled JPEG image [40, 39]. Based on this observation, for the considered scenario of matching a source point cloud with a set of database point clouds, it is preferable to compress and transmit 3D feature descriptors, instead of compressing and transmitting the whole point cloud $[100,102]$. There are three desirable characteristics while compressing 3D descriptors for online transfer:

- First, the compressed feature descriptors should be represented in less number of bits for efficient online transfer with low network latency. 
- Second, the compressed 3D feature descriptors should offer high keypoint matching accuracy to retrieve accurate results from the server's database.

- Third, the compressed descriptors should be matched directly without decompression, hence lowering the memory and computational power requirements on the end-devices.

\section{Challenges with existing real-valued and binary 3D feature descriptors:}

Existing real valued 3D feature descriptors that offer good keypoint matching performance require very high bandwidth for their transfer over the network. On the other hand, the existing binary 3D feature descriptor, B-SHOT, presented in the previous chapter, requires less bandwidth (352 bits per descriptor), when compared to real valued descriptors, however, it offers reduced keypoint matching performance. Moreover, when dealing with hundreds to thousands of descriptors extracted on a single point cloud, a handful of bits reduction per descriptor will have pronounced effect on the bandwidth requirements.

Contributions: In this chapter, we explore how low we can go in compressing real valued 3D feature descriptors via lattice quantization. We apply lattice quantization technique to compress three state-of-the-art 3D feature descriptors, namely, SHOT [128], RoPS [66] and FPFH [124] and present their achievable keypoint matching performance. We perform compressed domain matching, where the compressed feature descriptors are matched by querying a pre-computed look up table, which is significantly faster than the widely used kd-tree [126] based matching technique. We also propose a double stage lattice quantization to compress the SHOT feature descriptor even further. Based on the extensive evaluation on the Kinect dataset, we provide a spectrum of possible bitrates and achievable keypoint matching performances of these three feature descriptors with some interesting findings. This can help in selecting the appropriate compressed 3D feature descriptor for an application, as per its bandwidth and performance requirements. The source code will be made publicly available at https://sites.google.com/site/dslqshot/

\subsection{Related Work}

In this chapter, we use three state-of-the-art descriptors, SHOT [128], RoPS [66] and FPFH [124] extensively, whose design was briefly presented in Chapter 2. Moreover, in this chapter, we apply the proposed binarization technique in the previous chapter to RoPS and FPFH descriptors to create B-RoPS and B-FPFH descriptors and compare them with the created low bitrate 3D descriptors. Now, we present relevant works that create low bitrate variants of $2 \mathrm{D}$ and $3 \mathrm{D}$ feature descriptors via various compression techniques. 


\subsubsection{Compression Techniques in 2D Domain}

The process of standardizing compact 2D feature descriptors for visual search applications by MPEG $[38,39]$ has resulted in the development of various techniques to compress $2 \mathrm{D}$ feature descriptors, especially SIFT [90]. The final adopted technique in the MPEG standard is based on scalar quantization $[107,106,38]$, which employs small linear transforms and ternary scalar quantization to compress SIFT feature descriptor. The linear transforms and the selection of a subset of transformed descriptor values to be transmitted, were tailored/optimized specifically for SIFT feature descriptor. It is not intuitive to apply those linear transforms to 3D feature descriptors and choosing only a subset of the transformed values, because of the fundamental difference in the construction principles of SIFT and considered 3D feature descriptors, RoPS [66], FPFH [124] and SHOT [128].

The standard also adopted a multi-stage vector quantization [26] from various submitted proposals based on vector quantization. These vector quantization methods require a codebook that is learned from visual vocabularies, which is difficult in 3D domain for two main reasons. First, there is no standard or pre-built vocabularies that are publicly available. Second, it is not clear on how to accommodate the differences that arise from point cloud density variations and types of depth data, such as $2.5 \mathrm{D}$ and 3D.

In contrast to the previous methods, i.e., handcrafted scalar quantization, or vector quantization that requires pre-built vocabularies, there is lattice quantization technique [23, 24], which directly compresses normalized histograms or probability distributions based on a fixed codebook without any need for clustering or learning. Lattice quantization has been applied on 2D feature descriptors [24] for low bit rate image retrieval. Firstly, lattice quantization based compression has offered better performance when compared to Huffman and other tree based coding techniques $[58,23]$. Moreover, Huffman tree based coding technique has a major drawback, which is that the number of possible Huffman trees increase drastically with the number of bins in the histogram, necessitating the need for large memory to store those codebooks. Secondly, in lattice quantization, the codebook or the quantization levels are fixed based on the initially set parameters, and this enables compressed domain matching. Compressed domain matching directly matches the compressed descriptors with few look up table queries and hence there is no need to decompress the transmitted descriptors. After considering these advantages, we employed lattice quantization to compress 3D feature descriptors in this work, to achieve greater compression while offering good keypoint matching ${ }^{1}$ performance.

\footnotetext{
${ }^{1}$ By the term 'good keypoint matching performance', the authors refer to the performance in terms of both precision and recall among the compared descriptors.
} 


\subsubsection{Compressed 3D Feature Descriptors}

There was only a single attempt [91], as per our knowledge, to compress 3D feature descriptors for efficient online transfer. The authors tried to employ lattice quantization (also known as type coding) to compress 3D feature descriptors [91], however, there were many shortcomings in their proposal. First, they need to decompress the transmitted (compressed) feature descriptors to establish correspondences based on Euclidean distance metric. This means that the authors' proposal does not offer any reduction in memory footprint on both the source and the target devices and needs additional computational power for both compression and decompression. Second, they need to employ a specific software library [91] to handle extremely large integers of size 256 bits, which is not desirable to have, on mobile devices. Third, the highest compression that they have reported, required 528 bits to represent a 352 dimensional SHOT feature descriptor. Moreover, they complicate the lattice quantization process by appending the normalization factors at the end of the descriptors and it is not clear if their proposal can be applied to RoPS and FPFH feature descriptors as well. Finally, the computational requirements were not reported and it was only mentioned that the system requires significantly higher amount of time, which makes it is difficult to comprehend its practical use in real world applications.

We propose to employ lattice quantization to compress 3D feature descriptors in a much simpler way and overcome the above mentioned shortcomings of [91]. First, we perform compressed domain matching using a look up table and hence there is no need to decompress the compressed feature descriptors to find keypoint correspondences, thus offering significant reduction in memory footprint on end-devices. Second, our implementation does not require any additional software library, and we can compress a 352 dimensional SHOT feature descriptor to 176 bits (best performing compressed variant), thus requiring much lesser bandwidth, memory and power requirements. Moreover, we improve the conventional lattice quantizer by a double stage lattice quantization technique and show that it can offer even higher compression in the case of SHOT 3D descriptor. Also, we compress three state-of-the-art 3D feature descriptors, SHOT [128], RoPS [66] and FPFH [124] and perform rigorous evaluation on achievable bitrate and performance, and highlight the findings based on which, the user can choose the apt compressed 3D feature descriptor for their application. Finally, the compressed feature descriptors in our proposal require much lesser computational time than uncompressed original 3D feature descriptors, which makes them preferable even for on-device applications.

In the next section, we present lattice quantization and double stage lattice quantization, to compress 3D feature descriptors. 


\subsection{Lattice Quantization}

Scalar quantization can be seen as a function that maps the set of all real values to a finite set of output values, which forms the codebook. The cardinality of the codebook and the distribution of the output values determine the resolution and the quality of the performed quantization. Vector quantization is the generalized extension of scalar quantization to multiple dimensions. While scalar quantization is extensively used in analog to digital conversion, vector quantization, at times is used for data compression.

Lattice quantization is a special case of vector quantization where the codebook is regular, i.e., the real valued input vectors are quantized to a set of output vectors that are equally spaced in the representation space. The cardinality of the codebook determines the number of bits required to represent each possible output vector. All the possible output vectors in the codebook are encoded with a unique index, and this index alone is transmitted, hence drastically reducing the number of bits to represent a high dimensional vector.

\subsubsection{Lattice Quantization to Compress 3D Feature Descriptors}

Let us represent an $N$-dimensional 3D feature descriptor by $\mathbf{A}=\left\{a_{1}, a_{2}, \ldots, a_{N}\right\}$, which is formed by an aggregation of $N / m$ number of $m$-dimensional sub-vectors. These $m$-dimensional sub-vectors represent various properties that are repeatedly calculated around a keypoint. For example, a 352-dimensional SHOT feature descriptor is an aggregation of 32 number of 11dimensional histograms. Similarly, a 135-dimensional RoPS feature descriptor is made up of 27 number of 5-dimensional sub-vectors and a 33-dimensional FPFH feature descriptor is made of 3 number of 11-dimensional histograms. Let us represent the repeatable $m$ dimensional subvector by $\mathbf{B}=\left\{b_{1}, b_{2}, \ldots, b_{m}\right\}$ and $N / m$ such repeatable sub-vectors combine to form an $N$ dimensional 3D feature descriptor $\mathbf{A}$, where $\mathbf{A}=\left\{\mathbf{B}_{1}, \mathbf{B}_{2}, \ldots, \mathbf{B}_{N / m}\right\}$.

In lattice quantization, a single $m$-dimensional real valued sub-vector $\mathbf{B}$, is quantized and mapped to a specific vector in the codebook. Then a unique index representing the mapped vector in the codebook is transmitted over the network. Existing works [23, 24] on compressing $2 \mathrm{D}$ feature descriptors claim that, it is ideal to quantize and transmit a single sub-vector $\mathbf{B}$ at a time, which would result in transmission of $\frac{N}{m}$ indices for every $N$-dimensional feature descriptor. It was reported that there was no improvement in the performance when joint-histogram compression was employed to compress the 2D HOG descriptor [24]. In contrast to that, we have found that, in the case of 3D SHOT feature descriptor, a set of consecutive histograms can be compressed simultaneously, and this offers both higher compression and performance 
gain, when compared to single histogram compression. For example, we quantized two consecutive sub-vectors at a time, which would require transmission of only $\frac{N}{2 m}$ indices for every $N$-dimensional descriptor, and this had offered better performance while requiring less number of bits compared to single histogram compression, as shown in Experiment 1 later on.

Therefore, the parameter $m$ may not be limited to the length of a single repeatable subvector, and can be extended to accommodate consecutive sub-vectors, which results in much higher compression. Therefore, the parameter $m$ can take any value for which $\frac{N}{m}$ is always a natural number, thus it is not limited to the length of the repeatable sub-vector or its combinations. Another important parameter in lattice quantization is $n$, which determines the number of quantization levels in each dimension of the lattice. With an increase in $n$, the resolution of the quantized vector increases and makes them more distinctive. A codebook with high resolution quantized vectors requires more number of indices to encode them, resulting in greater number of bits to be transmitted per index.

Formally, a lattice quantization process initiated with $m$ and $n$ parameters, involves the construction of a lattice, where each point in the lattice is a $m$-dimensional probability distribution $\mathbf{Q}=\left\{q_{1}, q_{2}, . ., q_{m}\right\}$, and each quantity $q_{i}$ has $n$ quantization levels i.e.,

$$
q_{i}=\frac{c_{i}}{n}, \text { where }\left\{c_{i}, n\right\} \in Z_{+} \text {and } \sum_{i} c_{i}=n
$$

Example: A lattice constructed with parameters $m=5$ and $n=3$ consists of lattice points that span the space of all possible five dimensional probability distributions, where each dimension can have three possible states $\{0.33,0.66,1\}$, other than zero (from Eqn. 7.1). Some points from this lattice are $\{0.66,0.33,0,0,0\},\{0,0.33,0.33,0,0.33\}$, and $\{0,0,0,0,1\}$.

The constructed lattice is referred to as $A_{m-1}$ lattice and this technique is also referred to as lattice coding or type coding or $A_{m-1}$ lattice quantization in previous works [23, 24, 118]. The number of lattice points in the constructed lattice are given by the multiset coefficient:

$$
\left(\left(\begin{array}{c}
m \\
n
\end{array}\right)\right)=\left(\begin{array}{c}
n+m-1 \\
m-1
\end{array}\right)=\frac{(n+m-1) !}{(m-1) ! n !}
$$

Every input $m$-dimensional sub-vector $\mathbf{B}$, where $\mathbf{B}=\left\{b_{1}, b_{2}, \ldots, b_{m}\right\}$ is normalized to form a probability distribution $\overline{\mathbf{B}}=\left\{\overline{b_{1}}, \overline{b_{2}}, . ., \overline{b_{m}}\right\}$, where $\bar{b}_{i}=b_{i} / \sum b_{i}$. If the input sub-vector $\mathrm{B}$ has any negative values, then the largest of those negative values can be added to each element of $\mathbf{B}$, to make it non-negative and then normalization should be performed. After normalization, the resultant probability distribution $\overline{\mathbf{B}}$ is quantized to the closest lattice point $\mathbf{Q}=\left\{q_{1}, q_{2}, . ., q_{m}\right\}$ in the constructed lattice, based on the steps mentioned below in Algorithm 1. Then index of the mapped lattice point $\mathbf{Q}=\left\{q_{1}, q_{2}, . ., q_{m}\right\}$ is calculated based on 
lexicographic enumeration. Finally, $\log _{2}\left(\left(\begin{array}{c}m \\ n\end{array}\right)\right)$ bits (from Eqn. 7.2) are required to represent each index, with fixed length coding. Employing variable length coding can further reduce the number of bits required to represent the indices.

\section{Algorithm 1: To find the nearest lattice point}

We now present the algorithm $[118,24]$ to quantize a sub-vector $\mathbf{B}=\left\{b_{1}, b_{2}, . ., b_{m}\right\}$ to the nearest lattice point $\mathbf{Q}=\left\{q_{1}, q_{2}, . ., q_{m}\right\}$ in the lattice constructed with parameters $m$ and $n$, which represent the dimension of the input vector and the number of quantization levels in each dimension respectively.

1. Normalize the input sub-vector $\mathbf{B}$ to form a probability distribution $\overline{\mathbf{B}}=\left\{\overline{b_{1}}, \overline{b_{2}}, . ., \overline{b_{m}}\right\}$, where $\bar{b}_{i}=b_{i} / \sum b_{i}$.

2. Compute $\overline{c_{i}}$ for $(i=1,2, \ldots, m)$, where $\bar{c}_{i}=\left\lfloor n \bar{b}_{i}+\frac{1}{2}\right\rfloor$ and $n^{\prime}=\sum_{i} \bar{c}_{i}$.

3. If $n^{\prime}=n$, then the computed $\bar{c}_{i}$ values form the corresponding $q_{i}$ components of the quantized distribution $\mathrm{Q}$, which is the nearest lattice point.

4. If $n^{\prime} \neq n$, residual errors $e_{i}$ are computed, where $e_{i}=\overline{c_{i}}-n \overline{b_{i}}$, and $e_{i}$ are sorted in ascending order, i.e., $-\frac{1}{2} \leq e_{j_{1}} \leq e_{j_{2}} \leq \ldots \leq e_{j_{m}} \leq \frac{1}{2}$.

5. If $\delta>0$, where $\delta=n^{\prime}-n$, then $\delta$ values of $\bar{c}_{i}$ with largest errors are decremented, and the corresponding $q_{i}$ components of the quantized distribution $\mathbf{Q}$ are estimated as

$$
q_{j_{i}}= \begin{cases}\bar{c}_{j_{i}} & j=1,2, \ldots, m-\delta-1, \\ \bar{c}_{j_{i}}-1 & i=m-d, \ldots, m\end{cases}
$$

6. Else if $\delta<0$, then $|\delta|$ values of $\bar{k}_{i}$ with smallest errors are incremented, and the corresponding $q_{i}$ components of the quantized distribution $\mathrm{Q}$ are estimated as

$$
q_{j_{i}}=\left\{\begin{array}{lc}
\bar{c}_{j_{i}}+1 & i=1,2, \ldots,|\delta|, \\
\bar{c}_{j_{i}} & i=|\delta|+1, \ldots, m .
\end{array}\right.
$$




\section{Enumeration: Calculating the unique index}

The next step is to find a unique index $\alpha$ that corresponds to the lattice point $\mathbf{Q}=\left\{q_{1}, q_{2}, . ., q_{m}\right\}$ in the constructed lattice with $m$ and $n$. A lexicographic enumeration $[31,24,118]$ technique is employed to find the unique index $\alpha$ as:

$$
\alpha(\mathbf{Q})=\sum_{j=1}^{n-2} \sum_{i=0}^{q_{j}-1}\left(\left(\begin{array}{c}
m-j \\
n-i-\sum_{d=1}^{j-1} q_{d}
\end{array}\right)\right)+q_{n-1}
$$

This unique index $\alpha$ is estimated for every sub-vector $\mathbf{B}$ of the input 3D feature descriptor A. Please note that $\alpha$ is a whole number and it is bounded by the number of lattice points in the constructed lattice, as shown in Eqn. 7.2. The estimated indices $\alpha_{k}$ that correspond to $\mathbf{B}_{k}$, are concatenated to form the compressed feature descriptor corresponding to the original feature descriptor $\mathbf{A}$, where $k=1,2, \ldots, N / m, N$ is the dimension of the $3 \mathrm{D}$ feature descriptor $\mathbf{A}$ and $m$ represents the dimension of the sub-vector $\mathbf{B}_{k}$.

\subsubsection{Compressed Domain Matching}

The advantage of lexicographic enumeration [31] process is that, for a fixed lattice structure defined by $m$ and $n$, there is a one to one correspondence between the enumerated indices $\alpha_{k}$ and all the lattice points $Q_{k}$. Based on this observation, one can pre-compute the Euclidean distances between all pairs of lattice points and store them in a look up table. The look up table $(L U T)$ is a square matrix with each element representing the distance between the lattice points or the probability distributions that correspond to the indices of the $L U T$. The size of the $L U T$ depends on the number of lattice points (Eqn. 7.2) in the constructed lattice, as defined by parameters $m$ and $n$.

Finally, the distance between two feature descriptors $\mathbf{A}_{\beta}$ and $\mathbf{A}_{\gamma}$, whose compressed variants are represented by a set of indices $\left\{\beta_{1}, \beta_{2}, \ldots, \beta_{N / m}\right\}$ and $\left\{\gamma_{1}, \gamma_{2}, \ldots, \gamma_{N / m}\right\}$, can be easily calculated by accessing LUT as

$$
\operatorname{Dist}\left(\mathbf{A}_{\beta}, \mathbf{A}_{\gamma}\right)=\sum_{i=1}^{N / m} \operatorname{LUT}\left(\beta_{i}, \gamma_{i}\right)
$$

where $N$ is the dimension of $\mathbf{A}_{\beta}$ and $m$ is the dimension of sub-vector that is compressed via lattice quantization. Hence, the compressed feature descriptors, which are made up of the indices, can be directly matched in the compressed domain, without any need for decompression. Compressed domain matching is significantly faster than kd-tree based feature descriptor 


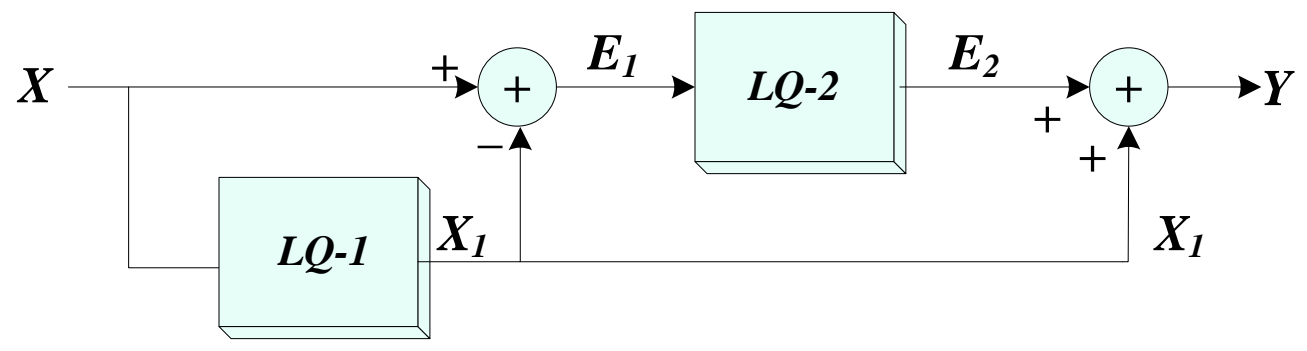

Figure 7.1: Flow chart of Double Stage Lattice Quantization (DSLQ)

matching, because the former requires few look up table queries and their summation to calculate their relative distance.

Though previous works $[23,24]$ in $2 \mathrm{D}$ feature descriptor compression have mentioned that the histogram based distance metrics such as KL divergence and Earth movers distance in the LUT offered higher number of keypoint correspondences, it was found that in matching 3D feature descriptors, Euclidean metric offered higher number of keypoint correspondences. The same observation that Euclidean metric offers better performance when compared to other distance metrics has also been made a survey [16] on 3D feature descriptors.

\subsubsection{Double Stage Lattice Quantization}

Gaining inspiration from the success of multistage vector quantization [26] in effectively quantizing the input vector, we propose double stage lattice quantization in this work. Please note that, in general, vector quantization involves learning and storage of codebooks while lattice quantization does not require those steps. Based on our knowledge, this is the first work to propose double stage lattice quantization (DSLQ) and evaluate its feasibility.

The basic idea behind DSLQ is to divide the quantization process into two stages. In the first stage, lattice quantization is performed on the original input vector, while in the second stage, the error vector between the input vector and the first stage output is quantized. The flowchart of DSLQ is shown in Fig. 7.1. Lets represent the input vector by $\mathbf{X}$, which is quantized by the first stage lattice quantizer $L Q-1$ and produces the quantized distribution $\mathbf{X}_{l}$. The second stage lattice quantizer $L Q-2$ operates on the vector difference $\mathbf{E}_{1}$, where $\mathbf{E}_{1}=\mathbf{X}-\mathbf{X}_{1}$, and generates the second stage quantized output $\mathbf{E}_{2}$. At last, the final quantized approximation of the input vector $\mathbf{X}$ is given by $\mathbf{Y}$, where $\mathbf{Y}=\mathbf{E}_{2}+\mathbf{X}_{1}$. Hence, DSLQ uses two indices, one from the first stage and the other from the second stage to represent the input vector.

Multistage vector quantization, in general, requires more number of bits to represent a vector, however, the quantization error is lower when compared to single stage vector quantization. 

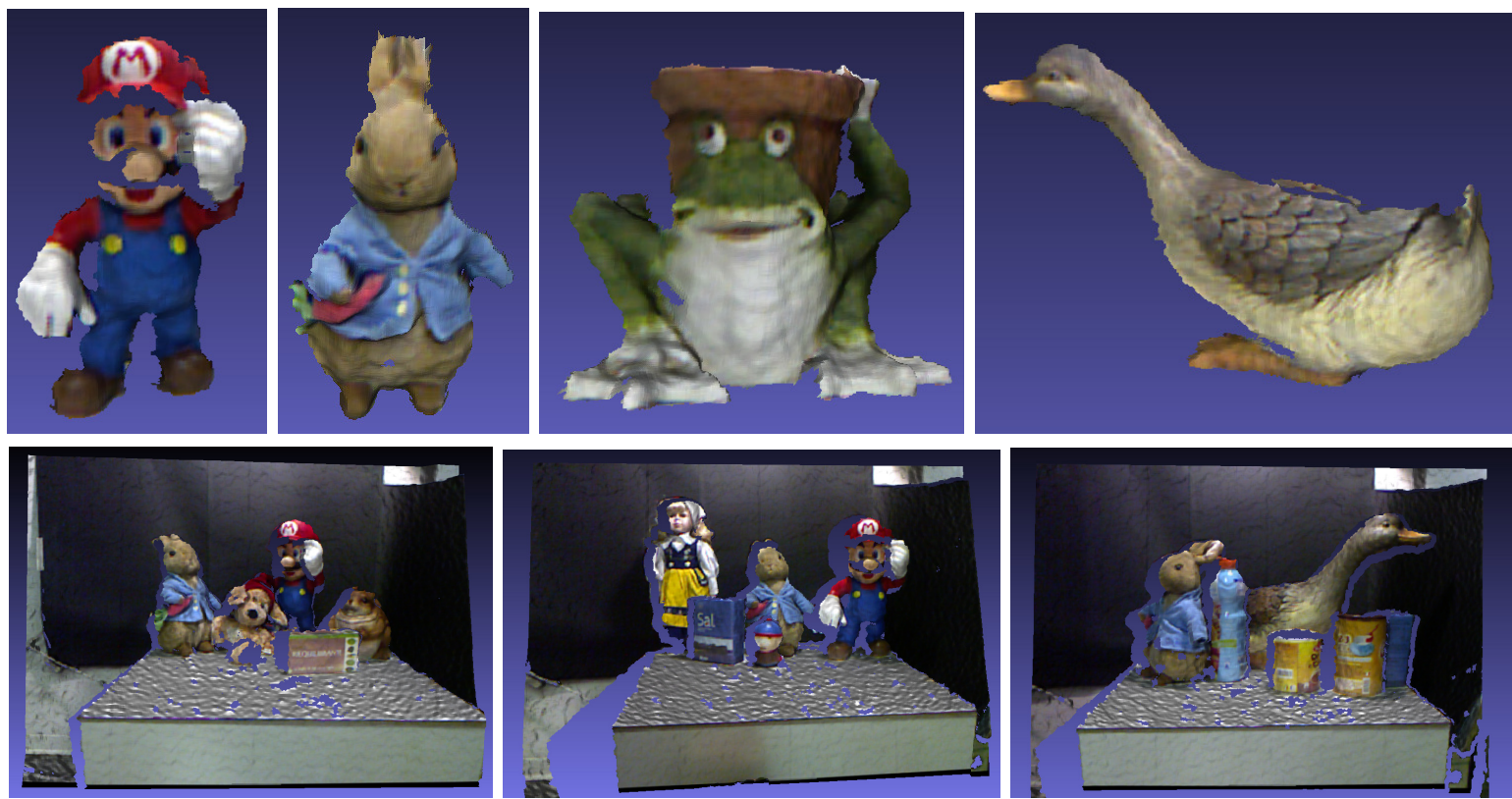

Figure 7.2: Various models and scenes from the Kinect dataset are shown here. The first row shows models named (from left to right) as mario000, PeterRabbit009, frog000 and duck009. The second row shows scenes named (from left to right) as scene003, scene005 and scene011.

In particular, we show in this work that, with appropriate choice of lattice quantization parameters ( $m$ and $n$ ), DSLQ can further compress the SHOT feature descriptor when compared to single stage lattice quantization as shown in Experiment 2 later on.

Please note that DSLQ based compressed feature descriptors are not matched in the compressed domain as in single stage lattice quantizers. This is because of the fact that, a better approximation to input vector is achievable with DSLQ, only when the two quantized distributions from both stages are reconstructed by summation on the decoder side. Hence, as in multistage vector quantization, queries to two vector tables and their summation is enough to reconstruct the final output vector from the transmitted indices. Then the reconstructed descriptors can be matched efficiently with kd-tree, resulting in the same computational complexity as traditional 3D feature descriptor matching. Another way to match DSLQ descriptors is to capitalize on the lexicographic enumeration process to generate the quantized distributions, which would save the memory as there wont be any need to store the vector tables; however, it would require additional computations to generate quantized distributions from transmitted indices. 


\subsection{Experimental Setup}

We employ the Kinect dataset ${ }^{2}$ that was used for evaluation of B-SHOT descriptor in the previous chapter, because of its high relevance [64] to real world applications. The depth data has similar kind of noise that is imminent in hand-held mobile devices such as Microsoft Kinect and Google Tango. This is also relevant in applications run on hand-held mobile devices or on autonomous robots equipped with sensors such as Microsoft Kinect and Asus Xtion Pro Live, that need to interact with a server. Moreover, innumerable applications for indoor mobile robots and $3 \mathrm{D}$ object recognition $[47,7]$ are developed using low-cost Kinect-style depth sensors.

\subsubsection{Evaluation Methodology}

We evaluate and compare the keypoint matching performance of the compressed and uncompressed 3D feature descriptors based on Precision Recall curves [64, 128, 66]. To generate precision recall curves, uniform keypoints are detected on scene, and with the available groundtruth, these scene keypoints are transformed onto the model. By employing uniform keypoints rather than random keypoints [128], we account for keypoints arising from all parts of the scene.

Next, detecting the keypoints on the scene and then finding their corresponding keypoints that only lie on the model, ensures that we account for all possible false positives that arise from the background of the scene and other object models. Once the keypoints on the model and scene are detected, the considered 3D descriptors for evaluation are extracted around these keypoints. A uniform keypoint detector with a radius $R_{k p}$ of $0.01 m$ was used while all the considered feature descriptors are extracted with a support radius $R_{f d}$ of $0.06 \mathrm{~m}$.

The exact steps to compute precision-recall curves have been presented in Sec. 5.3.2.1 and we follow the same here. For every model keypoint's feature descriptor, we find the first and the second nearest scene keypoint's feature descriptor. If the ratio of the distance of the first to the second nearest neighbour is less than or equal to a threshold $\alpha$, then the first nearest neighbour is considered as a correspondence. The threshold $\alpha$ is varied from 0 to 1 and accordingly the number of correspondences are calculated and the precision-recall are generated.

\section{Why Precision-Recall curve based evaluation and not $\operatorname{Robust} \operatorname{Recognition} \operatorname{Rate}(R R R)$ ?} It can be seen that Robust Recognition Rate $(R R R)$ metric boils down to a specific case of precision-recall curve based evaluation, when the threshold $\alpha=1$ and applying RANSAC on

\footnotetext{
${ }^{2}$ The dataset is available at http://vision.deis.unibo.it/research/80-shot
} 
the found correspondences to remove the false positives. This is because at $\alpha=1$, nearest neighbours are assigned as respective matches and applying a tight RANSAC algorithm to remove all false positives would only find true matches, equalling them to groundtruth matches. Next, as mentioned above, we import two salient characteristics of $R R R$ in to our precisionrecall curves based evaluation. First, we employ a uniform keypoint detector that caters for highest keypoint detection ambiguity. Second, we first detect the keypoints on the scene and then transform them on to the model, to cater for the false positives that arise from the background. Therefore, precision-recall curve based evaluation already considers $R R R$ metric and much more.

\subsection{Compressing Various 3D Feature Descriptors}

In this section, we compress three state-of-the-art 3D feature descriptors [64], SHOT [128], RoPS [66] and FPFH [124], and compare them based on the above mentioned evaluation methodology. As the amount of compression depends on the parameters $m$ and $n$, here, we experimentally determine the parameters that offer best keypoint matching performance and report the achievable compression.

\subsubsection{Compressing SHOT Feature Descriptor}

\section{Experiment 1: Single Stage Lattice Quantization of SHOT}

In this experiment, we compress SHOT feature descriptor by applying lattice quantization with varying $m$ and $n$ parameters. The $m$ and $n$ parameters determine the number of lattice points based on Eqn. 7.2, and hence, the number of bits required to represent the feature descriptor, i.e., achievable compression. As SHOT is an aggregation of 32 sub-vectors, where each subvector is a 11-bin histogram, we experimented by compressing each 11-bin histogram, i.e., $m$ is set to 11 and varied the parameter $n$. Then we explored if the consecutive histograms can be combined and compressed together in a single instance, hence we experimented with $m=\{22,44,88\}$. After fixing the parameter $m$, the value of the parameter $n$ is chosen in such a way that the number of lattice points in the constructed lattice is less than 8000 so that time and memory and computational requirements for lattice construction are tractable.

The results are shown in Fig. 7.4, where the compressed SHOT feature descriptors are shown in this representation, Type-SHOT (m,n) (ABC Bits), where (m,n) are the set $m$ and $n$ parameters while (ABC Bits) tells the number of bits required to represent the compressed descriptor via fixed-length coding. 

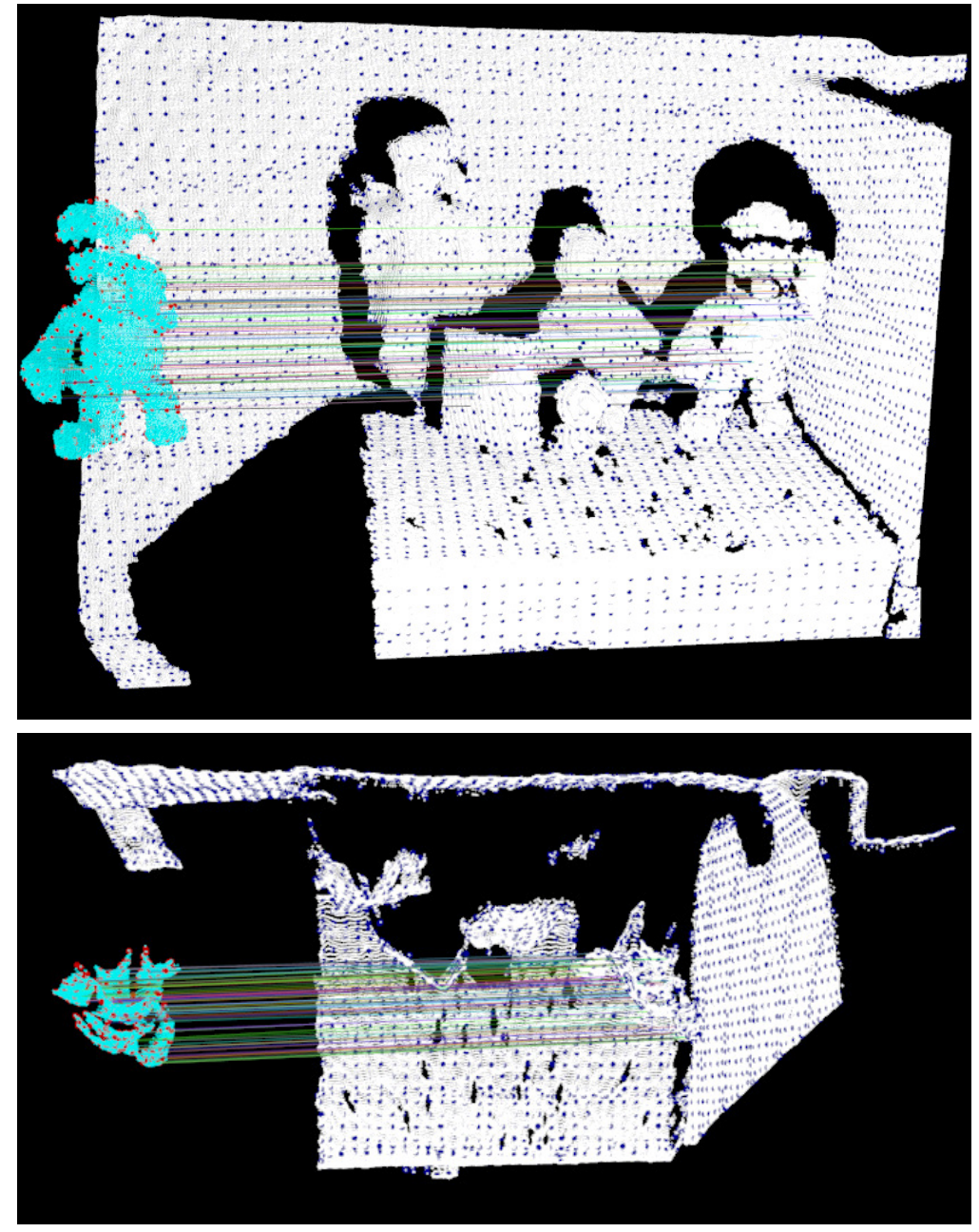

Figure 7.3: The front view and the top view are shown in this figure to illustrate the correspondences established with the Type-SHOT (22,3) (176 Bits) compressed descriptor between the model mario000 and the scene scene0 05 with keypoints extracted uniformly at $0.02 \mathrm{~m}$. The false positive matches are removed using the RANSAC algorithm.

It can be seen from Fig. 7.4 that Type-SHOT $(22,3)$ (176 Bits) is the most preferable compressed variant as it achieves consistently high recall and precision, specifically when compared to the higher bitrate variants such as Type-SHOT $(11,3)(288$ Bits) and Type-SHOT $(11,5)(384$ Bits). Existing works in 2D feature descriptor compression [23, 24] have reported that there was no improvement in the performance with joint histogram compression. In contrary to that observation, it was found that joint histogram compression with two consecutive 11-bin histograms, i.e., $m=22$ offers considerably better keypoint matching performance than TypeSHOT with $m=11$ on this dataset. Another observation is that if we try to compress SHOT even further than 176 bits with single stage lattice quantization, the performance of Type-SHOT drops as can be seen with other low bit variants such as Type-SHOT (22,2) (128 Bits) and TypeSHOT $(44,2)$ (80 Bits). Hence, with the use of single stage lattice quantization, SHOT feature 


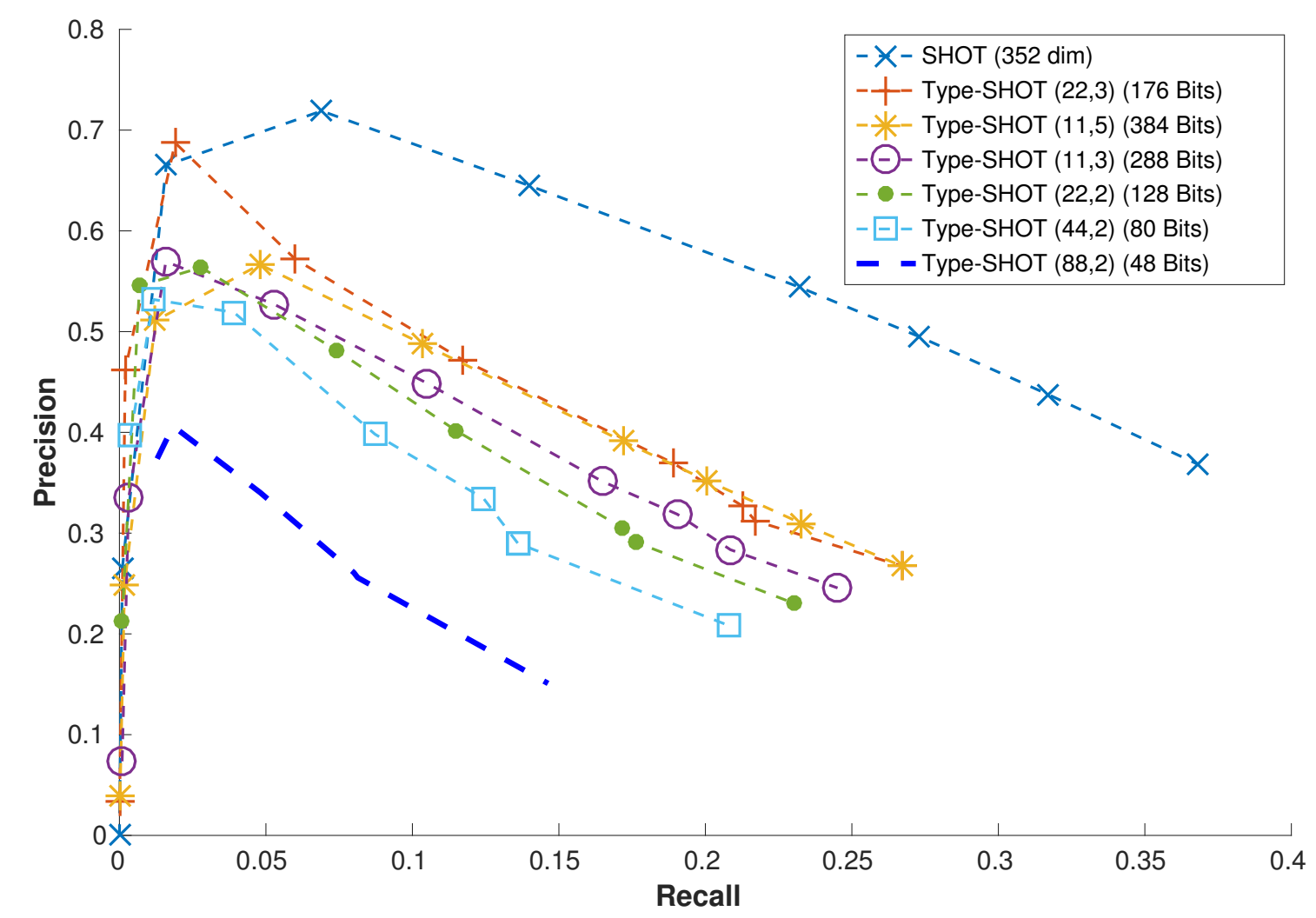

Figure 7.4: Performance evaluation of Type-SHOT feature descriptors with variation in $m$ and $n$ parameters as described in Experiment 1.

descriptor with 176 bits offers comparatively the best keypoint matching performance. As an example, in Fig. 7.3, we visualize the correspondences established by Type-SHOT $(22,3)$ (176 Bits) feature descriptors between the model mario000 and the scene scene005 from the Kinect dataset with keypoints extracted uniformly at a radius of $0.02 \mathrm{~m}$.

\section{Experiment 2: Double Stage Lattice Quantization of SHOT}

In this experiment, we apply double stage lattice quantization to compress SHOT feature descriptors even further, and compare the performance with single stage and B-SHOT binary 3D feature descriptor. We employed B-SHOT as it is the only publicly available low bitrate 3D feature descriptor. Please note that the original uncompressed SHOT descriptor requires 11,264 bits (based on IEEE floating point standard) while B-SHOT requires 352 bits.

The results are shown in Fig. 7.5. Double stage lattice quantization has two sets of $m$ and $n$ parameters. In Fig. 7.5, the double stage lattice quantized SHOT feature descriptors are represented as DSLQ-SHOT $\left(\mathrm{m}_{1}, \mathrm{n}_{1}\right)\left(\mathrm{m}_{2}, \mathrm{n}_{2}\right)$ (ABC Bits), where $\left(\mathrm{m}_{1}, \mathrm{n}_{1}\right)$ and $\left(\mathrm{m}_{2}, \mathrm{n}_{2}\right)$ are the $m$ and $n$ parameters of the first and second stages respectively, and (ABC Bits) tells the number of required bits. 


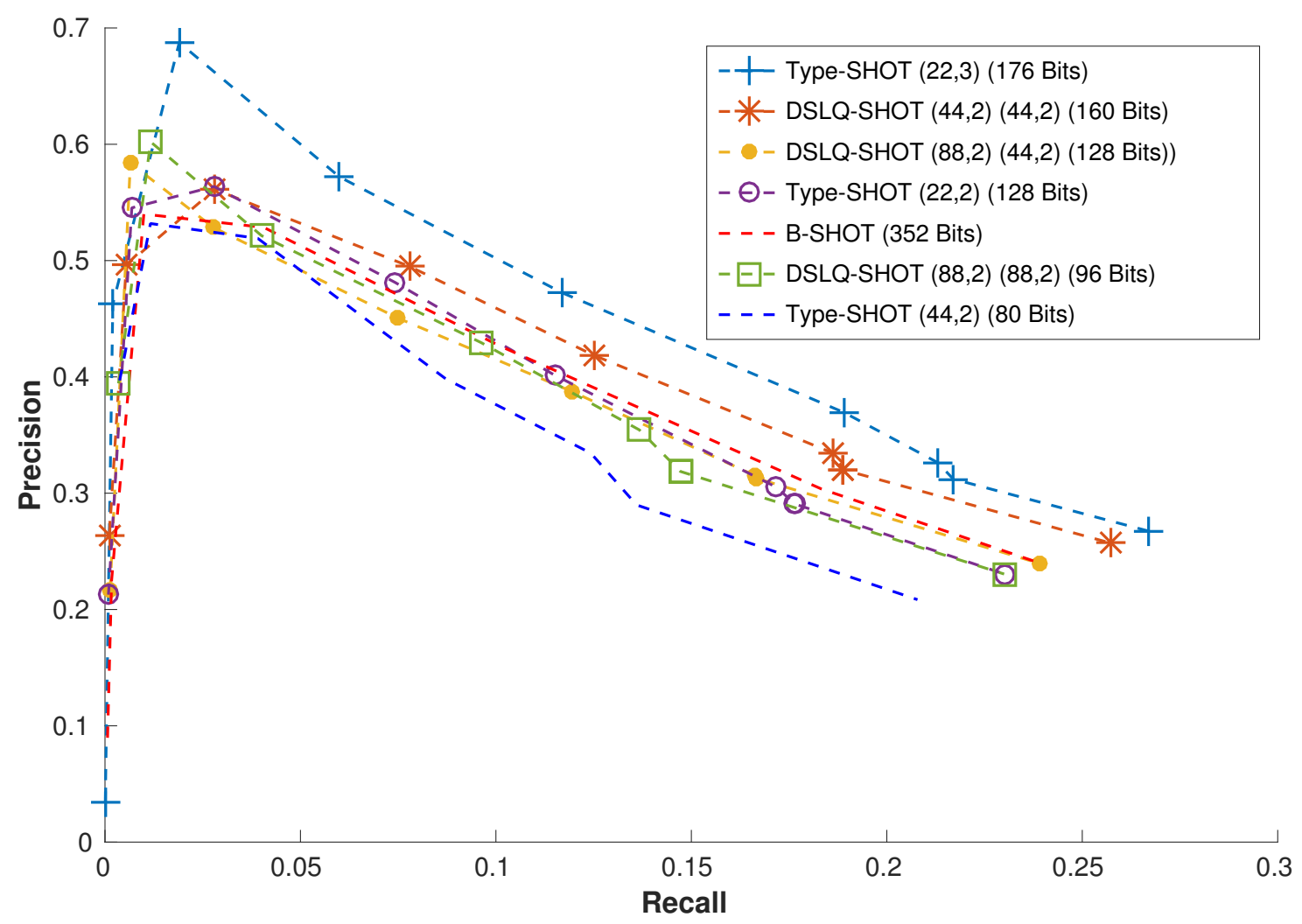

Figure 7.5: Performance evaluation of Type-SHOT and DSLQ-SHOT compressed descriptors as explained in Experiment 2.

From Fig. 7.5, DSLQ-SHOT $(44,2)(44,2)$ (160 Bits) offers the second best performance with Type-SHOT (22,3) (176 Bits) being the first. We consider the case where nearest neighbours are directly used as correspondences, i.e., $\alpha=1$, the point of highest recall in the figure. This is because, in most practical applications in $3 \mathrm{D}$, the first nearest neighbour is directly assigned as a match, rather than finding two nearest neighbours and thresholding their distance ratio. From those nearest neighbour matches, outliers can be easily removed via RANSAC, i.e., precision can be increased by post processing based on RANSAC. Hence, it is preferable to have more absolute inliers that are present at the high recall end. Though outliers appear at high recall, they can be removed using RANSAC, but is important to have more inliers(high recall), so that the transformation can be estimated in the first place. In such scenarios, where high recall is preferred, DSLQ-SHOT $(88,2)(44,2)$ (128 Bits) offered better performance than TypeSHOT $(22,2)$ (128 Bits). Hence, at a budget of 128 bits per SHOT descriptor, DSLQ-SHOT is preferable over Type-SHOT, highlighting the effectiveness of double stage lattice quantization. It can be seen from Fig. 7.5 that B-SHOT with 352 bits offers similar performance as that of DSLQ-SHOT at 128 bits. Again, DSLQ-SHOT $(88,2)(88,2)$ (96 Bits) offering similar performance as that of Type-SHOT $(22,2)$ (128 Bits) highlights the effectiveness of double stage 
lattice quantization in encoding the extra information in less number of bits. It can be noticed that further compression of SHOT with single stage lattice quantization, i.e., Type-SHOT $(44,2)$ (80 Bits) offers much lower performance because of the fact that performance drops at lower bitrate.

Double stage lattice quantization offers two reliable compressed SHOT variants that require 128 and 96 bits which offer better performance than Type-SHOT at 128 bits. While choosing the parameters $m$ and $n$ for DSLQ-SHOT, the parameter $n$ was set to 2 for $m=44$ and 88 , because for $n>2$, there is a drastic increase in both the lattice points and the size of the look up table, which needs to be stored for compressed domain matching of Type-SHOT descriptors. DSLQ-SHOT descriptors either require vector look up tables or decompression based on lexicographic enumeration to reconstruct the quantized vectors, which correspondingly require additional memory or computational time when compared to single stage lattice quantization. However, as this matching and decompression happens in the server, memory and computational requirements should not be a bottleneck. Hence, double stage lattice quantization can be employed to achieve further compression of SHOT while having a boost in the performance when compared to Type-SHOT at the same bitrate.

\subsubsection{Compressing RoPS Feature Descriptor}

\section{Experiment 3: Lattice Quantization of RoPS}

In this experiment, we compress RoPS feature descriptor with single stage lattice quantization. As RoPS descriptor is made up of five properties of a distribution matrix calculated 27 times, we first experimented by setting $m=5$ and then varied $n$. Second, RoPS, unlike SHOT and FPFH, is not an aggregation of histograms, hence, we also tried setting $m$ to factors of 135 , i.e., $m=\{3,5,9,15,27,45\}$ and then varied $n$. Hence, we report the results of the compressed RoPS variants with $m=\{5,9\}$, however, setting $m \geq 15$, needed much higher quantization levels $(n)$ to establish feature descriptor correspondences, and the lattice points in the constructed lattice were so high that they required larger memory to store the look up table for compressed domain matching, making them infeasible.

The results are shown in Fig. 7.6, where RoPS represents the original uncompressed feature descriptor that requires 4320 bits, B-RoPS that requires 135 bits is created by applying the adaptive quantization method proposed in B-SHOT [109] to RoPS feature descriptor, and TypeRoPS (m,n) (ABC Bits) represents a compressed variant of RoPS feature descriptor.

As can be seen from Fig. 7.6, Type-RoPS $(5,8)$ (243 Bits) offered relatively better keypoint matching performance compared to other compressed RoPS variants. As the bitrate decreases, 


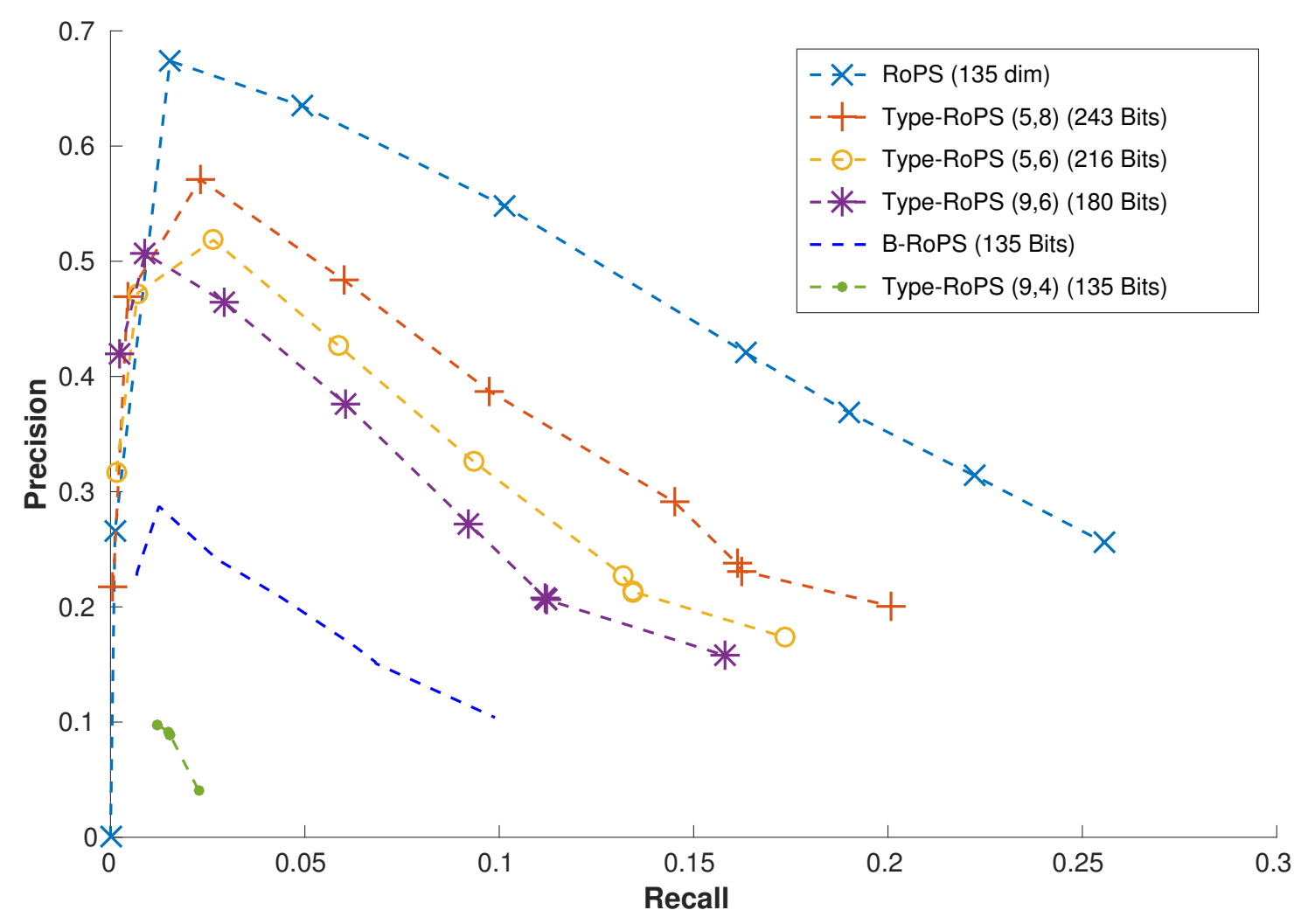

Figure 7.6: Performance evaluation of Type-RoPS feature descriptors with varying $m$ and $n$ parameters as explained in Experiment 3.

the performance also drops. However, it can be noted from the figure that at 135 bits, B-RoPS offered better performance than Type-RoPS $(9,4)(135$ Bits). This is because the number of quantization levels, i.e., $n=4$ is less and do not capture the variations in the RoPS descriptor. For example, Type-RoPS (5,8) (243 Bits) has eight quantization levels with $n=8$ and hence can capture the variations in the descriptor's values, resulting in high performing compressed RoPS variant. The reason behind this is that the RoPS descriptor is an aggregation of five statistical properties calculated over a matrix, which do not exhibit any resemblance with probability distributions. Hence, each value in RoPS descriptor can be very similar or close to each other, requiring higher precision/quantization levels to capture their variations and be discriminative from other compressed descriptors after normalization. From this experiment, it can be concluded that a single stage lattice quantized RoPS with 243 bits is the preferred compressed variant for RoPS descriptor.

The double stage lattice quantization (DSLQ) on RoPS descriptor did not offer more compression nor higher discriminative power. This is because RoPS descriptor is not made of histograms but statistical properties, which are similar to each other with less difference. Consequently, the normalization performed in the second stage in DSLQ renders the reconstructed 
RoPS descriptor to be less distinctive than a single stage lattice quantizer. The aim behind proposing DSLQ was to employ smaller lattice structures, so that less bits are required, and yet achieve good performance, which was not possible with the RoPS feature descriptor.

\subsubsection{Compressing FPFH Feature Descriptor}

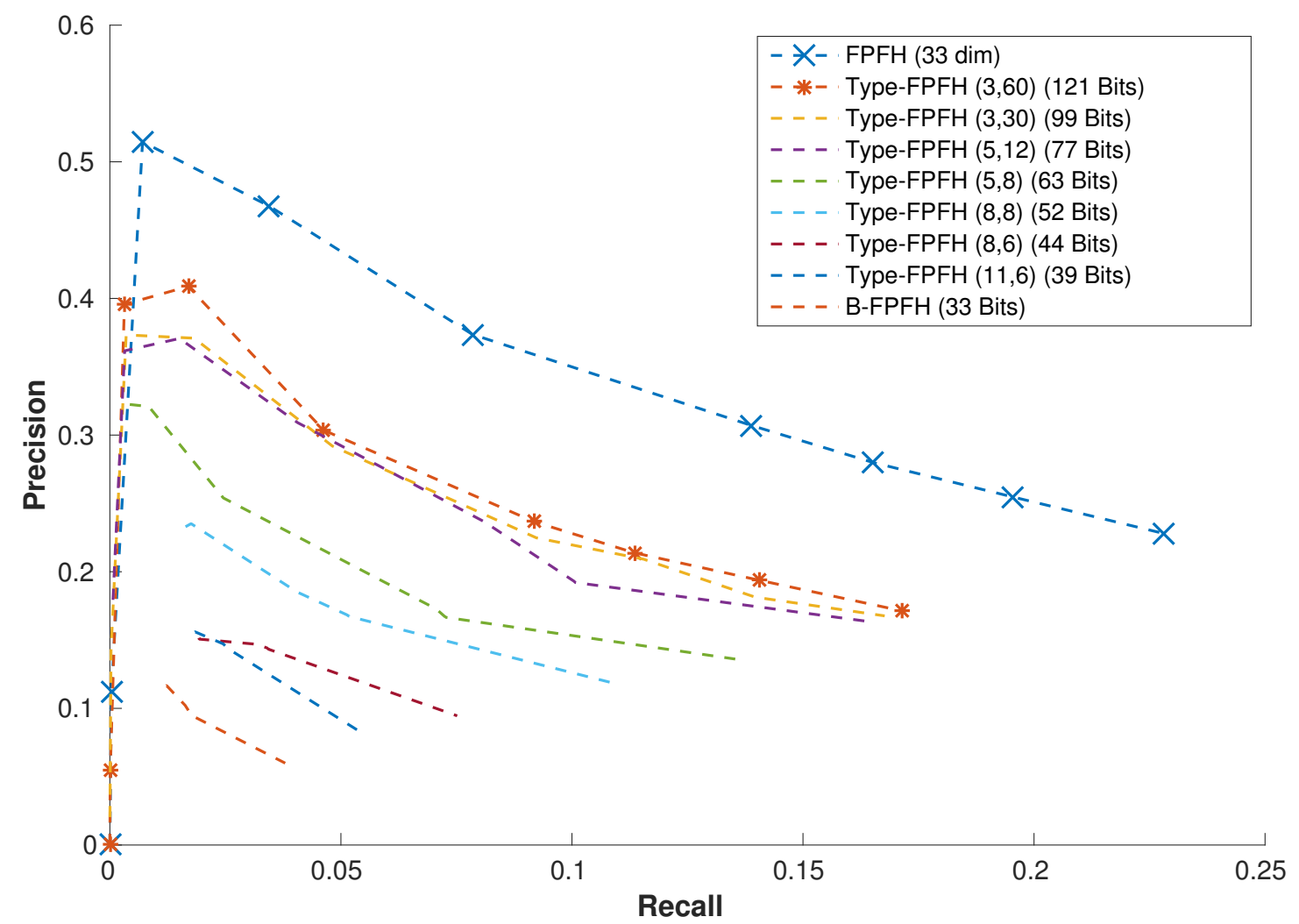

Figure 7.7: Performance evaluation of Type-FPFH feature descriptors with variation in $m$ and $n$ parameters as described in Experiment 4.

\section{Experiment 4: Lattice Quantization of FPFH}

We compress FPFH descriptor with the single stage lattice quantization technique. FPFH, being 33 dimensional, a combination of 3 histograms with 11 bins per each histogram, gives only two options for $m$, i.e., $m=\{3,11\}$. In order to have more compressed variants, we appended two zeros at the end of the 33 dimensional FPFH, which gave another possibility of $m=5$. Similarly, we only considered 32 dimensions generating another possibility of $m=8$. Therefore, we carried out experiments to evaluate the keypoint matching performance of the compressed variants of FPFH generated with $m=\{3,5,8,11\}$ and a correspondingly set $n$ value for which memory and computational requirements are tractable. For example, with $\{m, n\}=\{11,7\}$, the number of lattice points as calculated from Eqn. 3, are 19448, while for 
$\{m, n\}=\{11,6\}$, the number of lattice points are 8008 . Hence, we only evaluate till $n=6$ for a fixed value of $m=11$, as in this case, the memory and computational requirements for lattice construction and storage are significantly lower and tractable. B-FPFH, created by applying the adaptive quantization from B-SHOT [109] on to FPFH descriptor, is also considered for evaluation.

The results, as shown in Fig. 7.7, highlight that the performance of the compressed variants decrease accordingly with their bitrate. Type-FPFH (3,60) (121 Bits) offered high keypoint matching performance followed by Type-FPFH with 99, 77, 63 bits and so on. Type-FPFH $(11,6)$ with 39 bits offered better performance that B-FPFH with 33 bits, as can be seen from Fig. 7.7. Please note that the uncompressed FPFH descriptor requires 1056 bits for its representation, which shows that Type-FPFH (121 Bits) offers a compression ratio of $\approx 8.72$.

As seen in the compression of SHOT feature descriptor, double stage lattice quantizer was able to compress more than a single stage lattice quantizer, because it capitalized on compressing consecutive histograms with smaller lattice structures in both of its stages. This, consecutive histogram compression is not possible with FPFH descriptor as it is of 33 dimensional, with only three 11-bin histograms and a relatively smaller descriptor length, to see the advantage of double stage lattice quantization.

\subsubsection{Comparing the Compressed Variants of SHOT, RoPS and FPFH}

Here, in Fig. 7.8, we compare the best performing compressed variants of SHOT, RoPS and FPFH descriptors that were found in our previous experiments and the original uncompressed 3D feature descriptors. It can be seen from Fig. 7.8 that SHOT stands out as the best performing 3D feature descriptor. Next, for recall $<0.22$, RoPS performs better than Type-SHOT (176 Bits), however, for recall >0.22, Type-SHOT (176 Bits) outperforms RoPS. Also note that SHOT and RoPS require significantly higher memory requirements and feature descriptor matching time.

- Type-SHOT (176 Bits) is the best performing compressed descriptor and is also better than FPFH descriptor.

It can be said that DSLQ-SHOT (160 Bits) and DSLQ-SHOT (128 Bits) are the next best choices to have higher compression than 176 bits and yet achieve good keypoint matching performance. The uncompressed FPFP descriptor's performance comes next to DSLQ-SHOT (128 Bits). Type-RoPS (243 Bits) offered better performance than FPFH for recall $<0.13$, however, for recall $>0.13$ the uncompressed FPFH descriptor is better than Type-RoPS (243 Bits). 


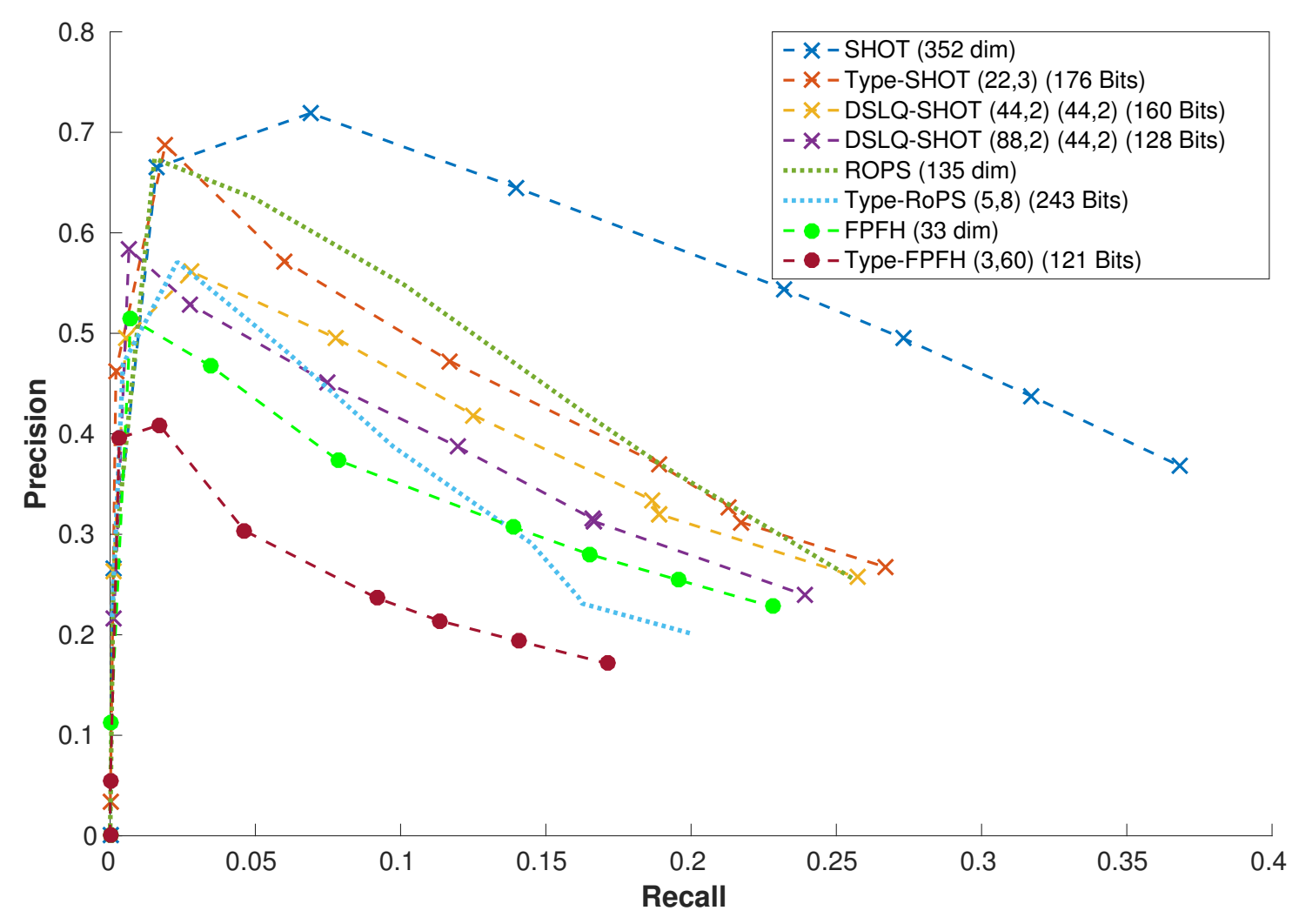

Figure 7.8: Comparison of the uncompressed and compressed variants of SHOT, RoPS and FPFH feature descriptors.

\subsection{Computational Requirements}

We present the computational time required to compute and match SHOT, B-SHOT and TypeSHOT (176 Bits) feature descriptors, as it offered highest keypoint matching performance. We considered a real world scenario where, detected keypoints are matched with feature descriptors and false positive matches are removed via RANSAC algorithm. Uniform keypoints were detected at $0.02 \mathrm{~m}$, considered feature descriptors were extracted with a support size of $0.05 \mathrm{~m}$ and the ransac inlier threshold was set to $0.04 \mathrm{~m}$. The reference implementation for SHOT and RANSAC are from the Point Cloud Library [126]. We employed a CPU with an Intel Xeon $(R)$ CPUE5-1650 $0 @ 3.20 \mathrm{GHz} \times 12$ and 16 GB RAM with UBUNTU 14.04 operating system for these experiments.

In this experiment, first, SHOT feature descriptors are extracted around the detected keypoints. Second, B-SHOT and Type-SHOT feature descriptors are calculated from SHOT and the time taken is shown in Fig. 7.9 as 'Computing from SHOT'. Third, reciprocal correspondences of SHOT are obtained using kdtree, while B-SHOT binary descriptors are matched via Hamming distance and Type-SHOT descriptors are matched in compressed domain. The 


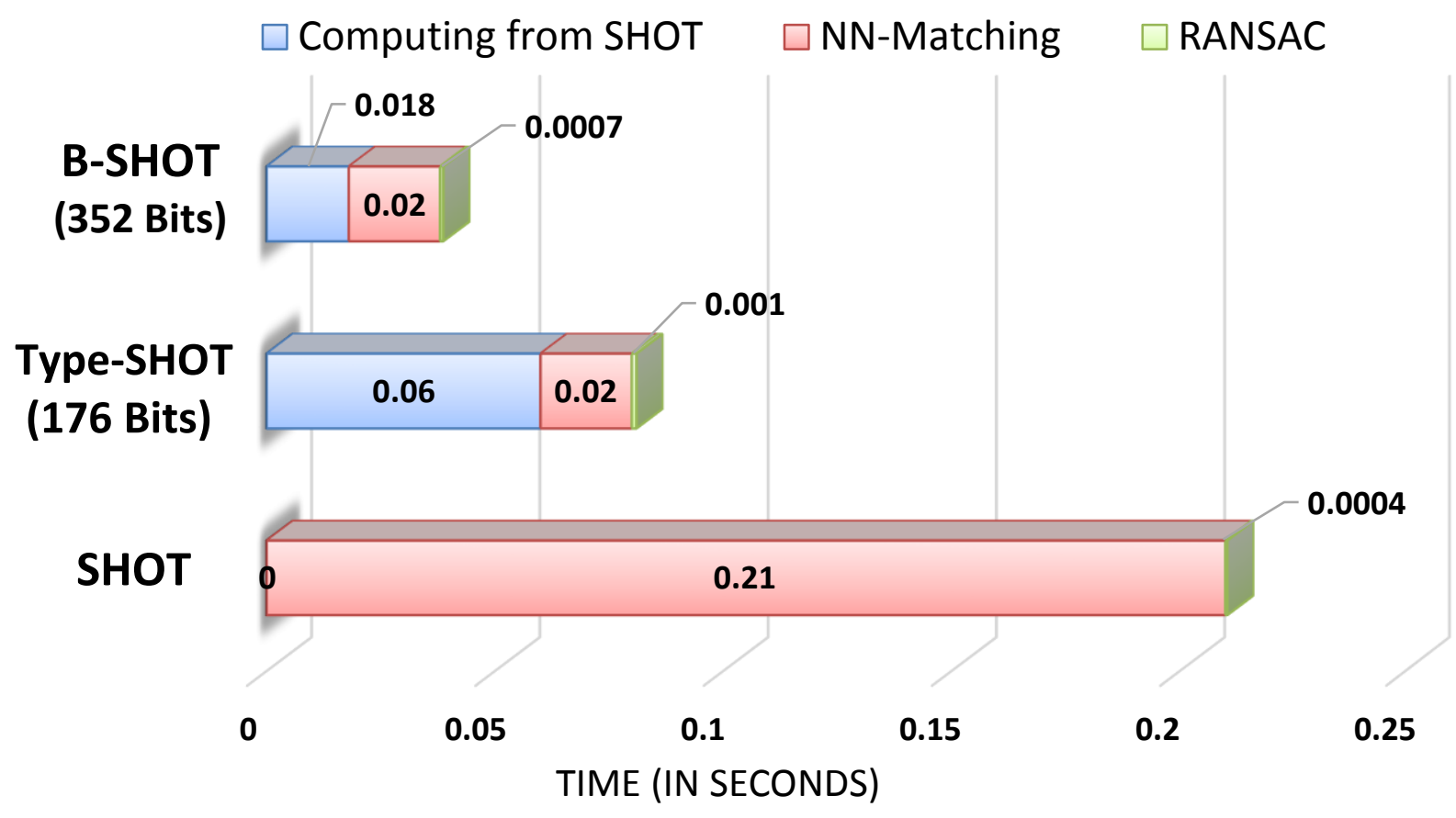

Figure 7.9: Comparing the computational time for B-SHOT, Type-SHOT (176 Bits) and SHOT feature descriptors.

corresponding time taken for matching is shown in Fig. 7.9 as 'NN-Matching'. Finally, the time taken by the RANSAC algorithm to find the true correspondences is shown in the figure as 'RANSAC'. Please note that all the reported values are averaged across all 49 scene-model pairs in the Kinect dataset.

It is evident from Fig. 7.9 that B-SHOT is the fastest, followed by Type-SHOT and SHOT feature descriptors. A closer look at the figure indicates that the NN-matching time of both BSHOT and Type-SHOT are equal and the difference arises only in computing the compressed variants. The fast $N N$-matching of Type-SHOT is justified by the fact that compressed domain matching involves only a few look up table queries and their summation to match two feature descriptors. Next, Type-SHOT takes more time for its creation from SHOT, when compared to B-SHOT, as it encodes much more relevant information through a relatively complex technique to achieve significantly higher keypoint matching performance. This experiment, highlights that Type-SHOT can also be employed in on-device applications as well to achieve faster matching with only a minimal loss in keypoint matching performance, when compared to original, uncompressed SHOT feature descriptor. 


\subsection{Conclusion}

In this chapter, we compressed three state-of-the-art 3D feature descriptors using lattice quantization and evaluated them on depth data acquired from Kinect-style sensors. More importantly, the compressed 3D feature descriptors can be directly matched in the compressed domain without any need for decompression, hence significantly reducing the memory footprint on the enddevices. We also proposed double stage lattice quantization to achieve even higher compression of SHOT 3D feature descriptor. It was found that the compressed SHOT feature descriptor at 176 bits offers best performance when compared to all other compressed variants and the uncompressed FPFH descriptor on the considered Kinect dataset. These compressed 3D feature descriptors have huge potential in applications such as 3D point cloud and object search, recognition and retrieval, as they, require significantly lower bandwidth for online transfer, are much faster in feature descriptor matching and have less memory footprint on end-devices. 


\section{Chapter 8}

\section{Conclusion and Future Work}

\subsection{Conclusion}

This thesis dealt with the proposal of salient 3D keypoint detectors and compact 3D feature descriptors for 3D perception applications. 3D keypoint detection and 3D feature description are the two most important steps in most of the 3D perception applications. The availability of affordable, hand-held and mobile 3D depth sensors such as Microsoft Kinect, Intel RealSense and Lenovo Phab2Pro smart phone enabled with Google Tango technology, insist the developed applications to be accurate, and efficient in terms of memory and computational resources.

Salient 3D keypoint detection: The first part of the thesis focussed on the design of salient 3D keypoint detectors. First, we proposed a keypoint detection algorithm that detects salient and repeatable keypoint sets by capitalizing on Histogram of Normal Orientations (HoNO). Second, we consider a specific 3D perception application, SLAM with an RGB-D camera, and proposed a new keypoint detection module that best suits the requirements, offering state-ofthe-art performance.

- Salient and repeatable keypoint sets via HoNO: Existing 3D keypoint detectors sometimes tend to detect keypoints on planar regions, or detect noisy glitches as keypoints, mainly because they do not employ robust criteria or they try to tackle two challenges in a single step. In our proposed method, we propose two independent steps, first to detect salient regions with efficient planar region removal and second to detect final keypoint sets from the salient regions. Histogram of Normal Orientations (HoNO), which represents the amount the surface variations, is proposed to effectively remove planar regions, based on its kurtosis, from the input point cloud. Next, the properties of HoNO and neighbourhood covariance matrix are employed to detect final keypoint sets. It is reliable to have repeatable keypoint sets at high curvature areas rather than having a single keypoint that might sometimes arise due to noise, or may lie in planar regions. 
- Keypoint based pose estimation from depth data: Sparse Depth Odometry (SDO)

We considered a 3D perception application that involves real time pose estimation of an RGB-D camera, solely from its depth data, and proposed a new keypoint detection module for this purpose. The new keypoint detection module enables real time and accurate pose estimation with Sparse Depth Odometry (SDO), as the keypoint detection module finds keypoints that are representative, offers accurate matches between frames with nearest neighbour association for fast matching. This new keypoint detection comprises of two existing keypoint detectors, SURE and NARF and they are selected based on extensive experimental and theoretical analysis. Finally, SDO powered with the proposed keypoint detection module offers competitive performance on standard RGB-D SLAM datasets, while working in real time with no GPU.

Compact 3D Feature Description: The second part of the thesis dealt with the proposals of a new real valued 3D feature descriptor, 3DHoPD, that is fast to compute. Then, to drastically lower the memory footprint and capitalize on Hamming distance based matching, the first binary 3D descriptor, B-SHOT is proposed. Next, we considered applications that require online transfer of $3 \mathrm{D}$ descriptors and proposed to employ lattice quantization to create even lower bitrate 3D descriptors that offer better performance than B-SHOT.

\section{- A fast low dimensional real valued 3D descriptor: 3DHoPD}

Existing real valued 3D feature descriptors are either high dimensional or demand more computational resources for their extraction and matching. We proposed 3DHoPD, a new low dimensional (18-dim) 3D feature descriptor that can be computed extremely fast, while offering competitive performance. Firstly, we proposed a new 3D keypoint transformation technique based on local reference frame. This technique transforms keypoints to a new 3D space where keypoints arising from similar surface patches lie close to each other. Secondly, a list of probable keypoint matches are grabbed in this new space, effectively removing up to $90 \%$ of false positive matches, thus making it easy to find the exact match. Lastly, a fast low dimensional descriptor, Histogram of Point Distributions (HoPD) is proposed to find the exact match from the retrieved list of probable matches.

\section{- The first binary 3D feature descriptor: B-SHOT}

There are many real valued 3D descriptors, however there is no binary 3D descriptor for keypoint matching on 3D point clouds. Hence, we proposed an adaptive binarization technique that converts a real valued 3D descriptor, SHOT to a binary descriptor, 
B-SHOT. B-SHOT offers 32 fold reduction in memory footprint while being $6 \mathrm{x}$ faster in descriptor matching when compared SHOT descriptor, however, B-SHOT offers a slightly reduced keypoint matching performance than SHOT, because of the fundamental reason that it encodes much lesser information than the latter.

\section{- Low bitrate 3D feature descriptors with lattice quantization}

While B-SHOT offered significant reduction in memory footprint and descriptor matching, it also offered reduced keypoint matching performance. In applications that require transfer of 3D descriptors online, it is required that the 3D descriptors have as low memory footprint as possible to handle large number of descriptors with lesser bandwidth, moreover, it is also necessary to retain their descriptive power as much as possible. Towards this direction, we applied lattice quantization to create even lower bitrate 3D descriptors than B-SHOT and yet offer improved performance. These low bitrate 3D descriptors can be matched in compressed without the need for decompression. We propose double stage lattice quantization to further compress SHOT descriptor. We also provide a spectrum of possible low bitrate variants and achievable performances of three state-of-the-art descriptors, SHOT, RoPS and FPFH.

\subsection{Future Research Directions}

There are three potential directions of research that stem out from the topics explored in this thesis. They are 3D keypoint detection, RGB-D odometry methods and 3D feature description.

\subsubsection{D Keypoint Detection:}

\section{- 3D keypoint matching with contextual information}

In Chapter 3, we proposed an algorithm to detect salient 3D keypoint sets, which essentially represent meaningful, high curvature regions or 'places' in the input point cloud. In general existing 3D feature descriptor based keypoint matching finds an exact match for a single keypoint. Instead, capitalizing on the detected keypoint sets that represent 'places' or regions, new 3D keypoint sets matching techniques can be proposed that combine multiple keypoint sets and match them in a single step, by considering contextual information [99, 12]. While this keypoint matching based on contextual information has been researched in 2D image domain $[132,116,84]$, it is yet to be explored in 3D. 


\section{- Learning based approaches for 3D keypoint detection}

Though there are couple of works that detect 3D keypoints on depth data using learning based approaches such as random forests [73] and boosting [32], still there is a need to develop more efficient and accurate learning based 3D keypoint detection techniques, that can be inspired from 2D image domain [98, 158, 151]. Towards this, a possible direction is to employ Deep Learning [83] for 3D keypoint detection, while generating labelled training data by employing multiple existing 3D keypoint detectors. As different keypoint detectors have varying criterion for keypoint detection, the learnt deep representations modelled on 3D data labelled with keypoints from multiple detectors may offer more robust and highly representative $3 \mathrm{D}$ keypoints.

\subsubsection{RGB-D Odometry Methods:}

\section{- Fusing Sparse and Dense RGB-D Odometry methods}

The research on developing accurate visual odometry methods is progressing in two directions. Firstly, sparse feature based odometry methods in which keypoints are tracked across frames to estimate pose, as can be seen by works such as ORB-SLAM [101] and RGBD-SLAM [43]. Secondly, dense methods are being pursued that do not detect keypoints, but employ gradient regions to minimize the photometric between consecutive frames and subsequently estimate the pose $[46,45]$. Sparse methods need texture in the environment but do not necessarily require consistent illumination, as the keypoint detectors can be robust to illumination variations. Dense methods do not require texture as they directly work on whole images or specific parts where there is gradient variations, but they require consistent illumination between frames. This clearly shows that combining sparse and dense methods for visual odometry would complement each other and result in accurate odometry systems with less failure cases.

\section{- Enhancing Sparse Depth Odometry with Bundle Adjustment}

The proposed 3D keypoint based pose estimation from dense depth data is efficient and accurate for small workspaces, however, SDO accumulates drift over time, due to noisy 3D keypoints. In SLAM literature [130, 56, 41], loop closure, either based on pose graph optimization or bundle adjustment is performed to reduce the drift when the same places are revisited. Here, we propose to employ bundle adjustment over pose graph optimization because it jointly optimizes both the pose information along with the detected 3D keypoints. This is better than pose graph optimization that solely optimizes the 
pose information alone. A local window based bundle adjustment can help in reducing the drift in odometry estimation while global bundle adjustment can perform large scale loop closures. Bundle adjustment requires accurate 3D keypoint correspondences, and here, the proposed low dimensional, binary and compressed 3D descriptors can prove to be extremely useful in establishing those accurate 3D keypoint correspondences with low memory and computational footprints.

\subsubsection{D Feature Description:}

\section{- Learning compact binary representations for 3D surface patches:}

Deep Learning has been extremely successful in 2D image domain for feature extraction $[115,159,52,131]$, where the deep learning based approaches outperformed conventional methods, mainly because of labelled training data and availability of huge computational resources. However, deep learning is still in its nascent stages in 3D because the number of convolutions and pooling operations show an exponential rise when moving from $2 \mathrm{D}$ to $3 \mathrm{D}$. There are few works where deep learning has been successful in specific applications such as 3D object recognition [42, 133, 95] or others [94], however, learning a generic $3 \mathrm{D}$ feature descriptor for $3 \mathrm{D}$ surface patches is yet to be explored.

There has been an attempt [161] to create a 3D descriptor based on deep learning, however, it had major shortcomings, such as huge computational time (few minutes to process one point cloud), as it employed signed distance function for surface representation. With the recent proposal of efficient 3D CNN [119], and directly working on raw 3D point clouds by capitalizing on the 3D keypoint transformation technique proposed in Chapter 5 , there is a possibility to learn deep representations of $3 \mathrm{D}$ surface patches, which can replace the existing handcrafted 3D feature descriptors such as SHOT and RoPS. 


\section{Bibliography}

[1] Project Tango, https://www.google.com/atap/project-tango/.

[2] Structure Sensor, http://structure.io/.

[3] Velodyne Lidar, http://velodynelidar.com/.

[4] Alexandre Alahi, Raphael Ortiz, and Pierre Vandergheynst. FREAK: Fast Retina Keypoint. In Computer Vision and Pattern Recognition (CVPR), 2012 IEEE Conference on, pages 510-517. IEEE, 2012.

[5] Andrea Albarelli, Emanuele Rodola, and Andrea Torsello. A Game-theoretic Approach to Fine Surface Registration without Initial Motion Estimation. In Computer Vision and Pattern Recognition (CVPR), 2010 IEEE Conference on, pages 430-437. IEEE, 2010.

[6] Andrea Albarelli, Emanuele Rodola, and Andrea Torsello. Loosely Distinctive Features for Robust Surface Alignment. In Computer Vision-ECCV 2010, pages 519-532. Springer, 2010.

[7] A. Aldoma, F. Tombari, L. Di Stefano, and M. Vincze. A Global Hypothesis Verification Framework for 3D Object Recognition in Clutter. Pattern Analysis and Machine Intelligence, IEEE Transactions on, PP(99):1-1, 2015.

[8] Aitor Aldoma, Zoltan-Csaba Marton, Federico Tombari, Walter Wohlkinger, Christian Potthast, Bernhard Zeisl, Radu Bogdan Rusu, Suat Gedikli, and Markus Vincze. Point cloud library: Three-Dimensional Object Recognition and 6 DoF Pose Estimation. IEEE Robotics \& Automation Magazine, 1070(9932/12), 2012.

[9] Alexandr Andoni and Piotr Indyk. Near-optimal hashing algorithms for approximate nearest neighbor in high dimensions. In 2006 47th Annual IEEE Symposium on Foundations of Computer Science (FOCS'06), pages 459-468. IEEE, 2006. 
[10] K.S. Arun, T. S. Huang, and S. D. Blostein. Least-Squares Fitting of Two 3-D Point Sets. Pattern Analysis and Machine Intelligence, IEEE Transactions on, PAMI-9(5):698-700, Sept 1987.

[11] Herbert Bay, Andreas Ess, Tinne Tuytelaars, and Luc Van Gool. Speeded-up Robust Features (SURF). Computer vision and image understanding, 110(3):346-359, 2008.

[12] F. Bellavia, D. Tegolo, and C. Valenti. Keypoint descriptor matching with context-based orientation estimation. Image and Vision Computing, 32(9):559 - 567, 2014.

[13] P. J. Besl and H. D. McKay. A Method for Registration of 3-D shapes. IEEE Transactions on Pattern Analysis and Machine Intelligence, 14(2):239-256, 1992.

[14] Michael Bosse and Robert Zlot. Place Recognition using Keypoint Voting in Large 3D Lidar Datasets. In Robotics and Automation (ICRA), 2013 IEEE International Conference on, pages 2677-2684. IEEE, 2013.

[15] Rasmus Bro, Evrim Acar, and Tamara G Kolda. Resolving the Sign Ambiguity in the Singular Value Decomposition. Journal of Chemometrics, 22(2):135-140, 2008.

[16] Anders G Buch, Henrik G Petersen, and Norbert Krüger. Local shape feature fusion for improved matching, pose estimation and 3d object recognition. SpringerPlus, 5(1):1, 2016.

[17] Erik Bylow, Jürgen Sturm, Christian Kerl, Fredrik Kahl, and Daniel Cremers. Real-time Camera Tracking and 3D Reconstruction using Signed Distance Functions. In Robotics Science and Systems (RSS), 2013.

[18] M. M. Bronstein C. Strecha, A. M. Bronstein and Pascal Fua. LDAHash: Improved Matching with Smaller Descriptors. IEEE Transactions on Pattern Analysis and Machine Intelligence, 34(1), 2012.

[19] M. Calonder, V. Lepetit, M. Ozuysal, T. Trzcinski, C. Strecha, and P. Fua. BRIEF: Computing a Local Binary Descriptor Very Fast. Pattern Analysis and Machine Intelligence, IEEE Transactions on, 34(7):1281-1298, July 2012.

[20] Michael Calonder, Vincent Lepetit, Christoph Strecha, and Pascal Fua. Brief: Binary Robust Independent Elementary Features. In Computer Vision-ECCV 2010, pages 778792. Springer, 2010. 
[21] D.R. Canelhas, T. Stoyanov, and A.J. Lilienthal. SDF Tracker: A Parallel Algorithm for On-line Pose Estimation and Scene Reconstruction from Depth Images. In Intelligent Robots and Systems (IROS), 2013 IEEE/RSJ International Conference on, pages 36713676, 2013.

[22] Umberto Castellani, Marco Cristani, Simone Fantoni, and Vittorio Murino. Sparse points matching by combining $3 \mathrm{~d}$ mesh saliency with statistical descriptors. In Computer Graphics Forum, volume 27, pages 643-652. Wiley Online Library, 2008.

[23] Vijay Chandrasekhar, Gabriel Takacs, DavidM. Chen, SamS. Tsai, Yuriy Reznik, Radek Grzeszczuk, and Bernd Girod. Compressed Histogram of Gradients: A Low-Bitrate Descriptor. International Journal of Computer Vision, 96(3):384-399, 2012.

[24] Vijay Ramaseshan Chandrasekhar. Low Bitrate Image Retrieval with Compressed Histogram of Gradients Descriptors. PhD thesis, Stanford University, 2013.

[25] Hui Chen and Bir Bhanu. 3D free-form Object Recognition in Range Images using Local Surface Patches. Pattern Recognition Letters, 28(10):1252-1262, 2007.

[26] Jie Chen, Ling-Yu Duan, Rongrong Ji, and Zhe Wang. Multi-stage Vector Quantization towards Low bit rate Visual Search. In Image Processing (ICIP), 2012 19th IEEE International Conference on, Sept 2012.

[27] Sungjoon Choi, Qian-Yi Zhou, and Vladlen Koltun. Robust Reconstruction of Indoor Scenes. In IEEE Conference on Computer Vision and Pattern Recognition (CVPR), 2015.

[28] Sungjoon Choi, Qian-Yi Zhou, Stephen Miller, and Vladlen Koltun. A Large Dataset of Object Scans. arXiv:1602.02481, 2016.

[29] Chin Seng Chua and Ray Jarvis. Point Signatures: A New Representation for 3D Object Recognition. International Journal of Computer Vision, 25(1):63-85, 1997.

[30] Ondrej Chum and Jiri Matas. Matching with PROSAC-Progressive Sample Consensus. In 2005 IEEE Computer Society Conference on Computer Vision and Pattern Recognition (CVPR'05), volume 1, pages 220-226. IEEE, 2005.

[31] T.M. Cover. Enumerative source encoding. Information Theory, IEEE Transactions on, 19(1):73-77, Jan 1973. 
[32] Clement Creusot, Nick Pears, and Jim Austin. A machine-learning approach to keypoint detection and landmarking on $3 \mathrm{~d}$ meshes. International journal of computer vision, 102(1-3):146-179, 2013.

[33] T. Darom and Y. Keller. Scale-Invariant Features for 3-D Mesh Models. Image Processing, IEEE Transactions on, 21(5):2758-2769, May 2012.

[34] Salvador Domínguez, Eduardo Zalama, Jaime Gómez García-Bermejo, Rainer Worst, and Sven Behnke. Fast 6D Odometry Based on Visual Features and Depth. In Frontiers of Intelligent Autonomous Systems, pages 5-16. Springer, 2013.

[35] Chitra Dorai and Anil K Jain. COSMOS-A Representation Scheme for 3D free-form Objects. Pattern Analysis and Machine Intelligence, IEEE Transactions on, 19(10):11151130, 1997.

[36] Chitra Dorai and Anil K. Jain. COSMOS-A Representation Scheme for 3D freeform Objects. Pattern Analysis and Machine Intelligence, IEEE Transactions on, 19(10):1115-1130, 1997.

[37] Ivan Dryanovski, Roberto G. Valenti, and Jizhong Xiao. Fast Visual Odometry and Mapping from RGB-D Data. In IEEE International Conference on Robotics and Automation (ICRA), 2013.

[38] L. Duan, V. Chandrasekhar, J. Chen, J. Lin, Z. Wang, T. Huang, B. Girod, and W. Gao. Overview of the MPEG-CDVS standard. Image Processing, IEEE Transactions on, PP(99):1-1, 2015.

[39] Ling-Yu Duan, Tiejun Huang, and Wen Gao. Overview of the MPEG CDVS Standard. In Data Compression Conference (DCC), 2015, pages 323-332, April 2015.

[40] Ling-Yu Duan, Jie Lin, Jie Chen, Tiejun Huang, and Wen Gao. Compact Descriptors for Visual Search. MultiMedia, IEEE, 21(3):30-40, July 2014.

[41] H. Durrant-Whyte and Tim Bailey. Simultaneous Localization and Mapping: Part I. Robotics Automation Magazine, IEEE, 13(2):99-110, June 2006.

[42] Andreas Eitel, Jost Tobias Springenberg, Luciano Spinello, Martin Riedmiller, and Wolfram Burgard. Multimodal deep learning for robust rgb-d object recognition. In Intelligent Robots and Systems (IROS), 2015 IEEE/RSJ International Conference on, pages 681-687. IEEE, 2015. 
[43] F. Endres, J. Hess, J. Sturm, D. Cremers, and W. Burgard. 3-D Mapping with an RGB-D Camera. Robotics, IEEE Transactions on, 30(1):177-187, Feb 2014.

[44] Felix Endres, Jürgen Hess, Nikolas Engelhard, Jürgen Sturm, Daniel Cremers, and Wolfram Burgard. An Evaluation of the RGB-D SLAM System. In in Proc. of IEEE International Conference on Robotics and Automation (ICRA), pages 1691-1696, 2012.

[45] Jakob Engel, Thomas Schöps, and Daniel Cremers. Lsd-slam: Large-scale direct monocular slam. In European Conference on Computer Vision, pages 834-849. Springer, 2014.

[46] Jakob Engel, Jörg Stückler, and Daniel Cremers. Large-scale direct slam with stereo cameras. In Intelligent Robots and Systems (IROS), 2015 IEEE/RSJ International Conference on, pages 1935-1942. IEEE, 2015.

[47] T. Faulhammer, A. Aldoma, M. Zillich, and M. Vincze. Temporal Integration of Feature Correspondences for Enhanced Recognition in Cluttered and Dynamic Environments. In Robotics and Automation (ICRA), 2015 IEEE International Conference on, pages 3003-3009, May 2015.

[48] Silvio Filipe and Lu1s A Alexandre. A Comparative Evaluation of 3D Keypoint Detectors. In 9th Conference on Telecommunications, Conftele, pages 145-148, 2013.

[49] Sílvio Filipe and Luís A Alexandre. A Comparative Evaluation of 3D Keypoint Detectors in a RGB-D Object Dataset. In Computer Vision Theory and Applications (VISAPP), 2014 International Conference on, volume 1, pages 476-483. IEEE, 2014.

[50] Silvio Filipe, Laurent Itti, and Luís A Alexandre. BIK-BUS: Biologically Motivated 3D Keypoint based on Bottom-up Saliency. IEEE Transactions on Image Processing, 24(1):163-175, 2015.

[51] Torsten Fiolka, Jörg Stückler, Dominik A Klein, Dirk Schulz, and Sven Behnke. SURE: Surface Entropy for Distinctive 3D Features. In Spatial Cognition VIII, pages 74-93. Springer, 2012.

[52] Philipp Fischer, Alexey Dosovitskiy, and Thomas Brox. Descriptor matching with convolutional neural networks: a comparison to sift. arXiv preprint arXiv:1405.5769, 2014.

[53] Martin A. Fischler and Robert C. Bolles. Random Sample Consensus: A Paradigm for Model Fitting with Applications to Image Analysis and Automated Cartography. Commun. ACM, 24(6):381-395, June 1981. 
[54] A Flint, A Dick, and A Van den Hengel. Local 3D Structure Recognition in Range Images. IET Computer Vision, 2(4):208-217, 2008.

[55] Alex Flint, Anthony Dick, and Anton van den Hengel. Thrift: Local 3D Structure Recognition. In 9th Biennial Conference of the Australian Pattern Recognition Society on Digital Image Computing Techniques and Applications (DICTA 2007).

[56] Friedrich Fraundorfer and Davide Scaramuzza. Visual Odometry: Part II: Matching, Robustness, Optimization, and Applications. Robotics \& Automation Magazine, IEEE, 19(2):78-90, 2012.

[57] Andrea Frome, Daniel Huber, Ravi Kolluri, Thomas Bülow, and Jitendra Malik. Recognizing Objects in Range Data using Regional Point Descriptors. In European conference on computer vision, pages 224-237. Springer, 2004.

[58] Travis Gagie. Compressing Probability Distributions. Information Processing Letters, 97(4):133 - 137, 2006.

[59] D. Galvez-LoİĄpez and J.D. Tardos. Bags of Binary Words for Fast Place Recognition in Image Sequences. Robotics, IEEE Transactions on, 28(5):1188-1197, Oct 2012.

[60] Ross Girshick, Jeff Donahue, Trevor Darrell, and Jitendra Malik. Rich feature hierarchies for accurate object detection and semantic segmentation. In Proceedings of the IEEE conference on computer vision and pattern recognition, pages 580-587, 2014.

[61] Yunchao Gong, Svetlana Lazebnik, Albert Gordo, and Florent Perronnin. Iterative Quantization: A Procrustean Approach to learning Binary Codes for Large-scale Image Retrieval. Pattern Analysis and Machine Intelligence, IEEE Transactions on, 35(12):29162929, 2013.

[62] M. Gordon, M. Hebel, and M. Arens. A Descriptor and Voting Scheme for Fast 3D Self-Localization in Man-Made Environments. In 2016 13th Conference on Computer and Robot Vision (CRV), pages 319-326, June 2016.

[63] Yulan Guo, M. Bennamoun, F. Sohel, Min Lu, and Jianwei Wan. 3D Object Recognition in Cluttered Scenes with Local Surface Features: A Survey. Pattern Analysis and Machine Intelligence, IEEE Transactions on, 36(11):2270-2287, Nov 2014. 
[64] Yulan Guo, Mohammed Bennamoun, Ferdous Sohel, Min Lu, Jianwei Wan, and NgaiMing Kwok. A Comprehensive Performance Evaluation of 3D Local Feature Descriptors. International Journal of Computer Vision, pages 1-24, 2015.

[65] Yulan Guo, F. Sohel, M. Bennamoun, Jianwei Wan, and Min Lu. An Accurate and Robust Range Image Registration Algorithm for 3D Object Modeling. Multimedia, IEEE Transactions on, 16(5):1377-1390, Aug 2014.

[66] Yulan Guo, Ferdous Sohel, Mohammed Bennamoun, Min Lu, and Jianwei Wan. Rotational Projection Statistics for 3D Local Surface Description and Object Recognition. International Journal of Computer Vision, 105(1):63-86, 2013.

[67] Yulan Guo, Ferdous Sohel, Mohammed Bennamoun, Jianwei Wan, and Min Lu. A novel local surface feature for $3 \mathrm{~d}$ object recognition under clutter and occlusion. Information Sciences, 293:196 - 213, 2015.

[68] Yulan Guo, Ferdous Ahmed Sohel, Mohammed Bennamoun, Min Lu, and Jianwei Wan. TriSI: A Distinctive Local Surface Descriptor for 3D Modeling and Object Recognition. In GRAPP/IVAPP, pages 86-93, 2013.

[69] Saurabh Gupta, Ross Girshick, Pablo Arbeláez, and Jitendra Malik. Learning rich features from rgb-d images for object detection and segmentation. In European Conference on Computer Vision, pages 345-360. Springer, 2014.

[70] Jungong Han, Ling Shao, Dong Xu, and Jamie Shotton. Enhanced Computer Vision with Microsoft Kinect Sensor: A Review. IEEE transactions on cybernetics, 43(5):1318$1334,2013$.

[71] Peter Henry, Michael Krainin, Evan Herbst, Xiaofeng Ren, and Dieter Fox. RGB-D mapping: Using Kinect-style depth cameras for dense 3D modeling of indoor environments. The International Journal of Robotics Research, 31(5):647-663, 2012.

[72] Huy Tho Ho and Danny Gibbins. Multi-Scale Feature Extraction for 3D Models using Local Surface Curvature. In Digital Image Computing: Techniques and Applications (DICTA), 2008, pages 16-23. IEEE, 2008.

[73] Stefan Holzer, Jamie Shotton, and Pushmeet Kohli. Learning to efficiently detect repeatable interest points in depth data. In Computer Vision-ECCV 2012, volume 7572 of Lecture Notes in Computer Science, pages 200-213. Springer Berlin Heidelberg, 2012. 
[74] Armin Hornung, Kai M Wurm, Maren Bennewitz, Cyrill Stachniss, and Wolfram Burgard. OctoMap: An Efficient Probabilistic 3D Mapping Framework based on Octrees. Autonomous Robots, pages 1-18, 2013.

[75] Linjia Hu and Saeid Nooshabadi. G-SHOT: GPU Accelerated 3D Local Descriptor for Surface Matching. Journal of Visual Communication and Image Representation, 30:343-349, 2015.

[76] Albert S Huang, Abraham Bachrach, Peter Henry, Michael Krainin, Daniel Maturana, Dieter Fox, and Nicholas Roy. Visual odometry and Mapping for Autonomous Flight using an RGB-D camera. In Interantional Symposium on Robotics Research (ISRR), 2011.

[77] Yangqing Jia, Evan Shelhamer, Jeff Donahue, Sergey Karayev, Jonathan Long, Ross Girshick, Sergio Guadarrama, and Trevor Darrell. Caffe: Convolutional architecture for fast feature embedding. In Proceedings of the 22nd ACM international conference on Multimedia, pages 675-678. ACM, 2014.

[78] Andrew E. Johnson and Martial Hebert. Using Spin Images for Efficient Object Recognition in Cluttered 3D Scenes. Pattern Analysis and Machine Intelligence, IEEE Transactions on, 21(5):433-449, 1999.

[79] Christian Kerl, Jürgen Sturm, and Daniel Cremers. Robust Odometry Estimation for RGB-D Cameras. In IEEE Interantional Conference on Robotics and Automation, ICRA, 2013.

[80] Kourosh Khoshelham and Sander Oude Elberink. Accuracy and resolution of kinect depth data for indoor mapping applications. Sensors, 12(2):1437-1454, 2012.

[81] Jan Knopp, Mukta Prasad, Geert Willems, Radu Timofte, and Luc Van Gool. Hough Transform and 3D SURF for Robust Three Dimensional Classification. In Computer Vision-ECCV 2010, pages 589-602. Springer, 2010.

[82] Alex Krizhevsky, Ilya Sutskever, and Geoffrey E Hinton. Imagenet Classification with Deep Convolutional Neural Networks. In Advances in neural information processing systems, pages 1097-1105, 2012.

[83] Yann LeCun, Yoshua Bengio, and Geoffrey Hinton. Deep learning. Nature, 521(7553):436-444, 2015. 
[84] Sunmin Lee and Yong Cheol Kim. Two-step clustering of sift keypoints and relaxation based matching of clusters. In Proceedings of the 2015 Conference on research in adaptive and convergent systems, pages 200-204. ACM, 2015.

[85] Stefan Leutenegger, Margarita Chli, and Roland Yves Siegwart. BRISK: Binary Robust Invariant Scalable Keypoints. In Computer Vision (ICCV), 2011 IEEE International Conference on, pages 2548-2555. IEEE, 2011.

[86] Stefan Leutenegger, Simon Lynen, Michael Bosse, Roland Siegwart, and Paul Furgale. Keyframe-based Visual-Inertial Odometry using Nonlinear Optimization. The International Journal of Robotics Research, 34(3):314-334, 2015.

[87] Kevin Lin, Jiwen Lu, Chu-Song Chen, and Jie Zhou. Learning compact binary descriptors with unsupervised deep neural networks. In Computer Vision and Pattern Recognition, 2016. CVPR 2016. IEEE Conference on. IEEE, 2016.

[88] Ziwei Liu, Xiaoxiao Li, Ping Luo, Chen-Change Loy, and Xiaoou Tang. Semantic image segmentation via deep parsing network. In Proceedings of the IEEE International Conference on Computer Vision, pages 1377-1385, 2015.

[89] Jonathan Long, Evan Shelhamer, and Trevor Darrell. Fully convolutional networks for semantic segmentation. In Proceedings of the IEEE Conference on Computer Vision and Pattern Recognition, pages 3431-3440, 2015.

[90] David G Lowe. Distinctive Image Features from Scale-Invariant Keypoints. International journal of computer vision, 60(2):91-110, 2004.

[91] F. Malaguti, F. Tombari, S. Salti, D. Pau, and L. Di Stefano. Toward Compressed 3D Descriptors. In 3D Imaging, Modeling, Processing, Visualization and Transmission (3DIMPVT), 2012 Second International Conference on, pages 176-183, Oct 2012.

[92] Zoltan-Csaba Marton, Dejan Pangercic, Nico Blodow, and Michael Beetz. Combined 2D-3D Categorization and Classification for Multimodal Perception Systems. The International Journal of Robotics Research, 30(11):1378-1402, 2011.

[93] Zoltan-Csaba Marton, Dejan Pangercic, Nico Blodow, Jonathan Kleinehellefort, and Michael Beetz. General 3D Modelling of Novel Objects from a Single View. In Intelligent Robots and Systems (IROS), 2010 IEEE/RSJ International Conference on, pages 3700-3705. IEEE, 2010. 
[94] Daniel Maturana and Sebastian Scherer. 3d convolutional neural networks for landing zone detection from lidar. In 2015 IEEE International Conference on Robotics and Automation (ICRA), pages 3471-3478. IEEE, 2015.

[95] Daniel Maturana and Sebastian Scherer. Voxnet: A 3d convolutional neural network for real-time object recognition. In Intelligent Robots and Systems (IROS), 2015 IEEE/RSJ International Conference on, pages 922-928. IEEE, 2015.

[96] A. Mian, M. Bennamoun, and R. Owens. On the Repeatability and Quality of Keypoints for Local Feature-based 3D Object Retrieval from Cluttered Scenes. International Journal of Computer Vision, 89(2-3):348-361, 2010.

[97] Krystian Mikolajczyk and Cordelia Schmid. A Performance Evaluation of Local Descriptors. Pattern Analysis and Machine Intelligence, IEEE Transactions on, 27(10):1615-1630, 2005.

[98] Kwang Moo Yi, Yannick Verdie, Pascal Fua, and Vincent Lepetit. Learning to assign orientations to feature points. In Conference on Computer Vision and Pattern Recognition (CVPR), pages 107-116. IEEE, 2016.

[99] Brittany Morago, Giang Bui, and Ye Duan. An ensemble approach to image matching using contextual features. IEEE Transactions on Image Processing, 24(11):4474-4487, 2015.

[100] Vicente Morell, Sergio Orts, Miguel Cazorla, and Jose Garcia-Rodriguez. Geometric 3D Point Cloud Compression. Pattern Recognition Letters, 50:55 - 62, 2014. Depth Image Analysis.

[101] Raul Mur-Artal, JMM Montiel, and Juan D Tardós. Orb-slam: a versatile and accurate monocular slam system. IEEE Transactions on Robotics, 31(5):1147-1163, 2015.

[102] Javier Navarrete, Vicente Morell, Miguel Cazorla, Diego Viejo, Jose Garcea-Rodreguez, and Sergio Orts-Escolano. 3DCOMET: 3D Compression Methods Test Dataset. Robotics and Autonomous Systems, pages -, 2015.

[103] Richard A Newcombe, Andrew J Davison, Shahram Izadi, Pushmeet Kohli, Otmar Hilliges, Jamie Shotton, David Molyneaux, Steve Hodges, David Kim, and Andrew Fitzgibbon. KinectFusion: Real-time Dense Surface Mapping and Tracking. In IEEE International Symposium on Mixed and augmented reality (ISMAR), pages 127-136, 2011. 
[104] John Novatnack and Ko Nishino. Scale-Dependent/Invariant Local 3D Shape Descriptors for Fully Automatic Registration of Multiple Sets of Range Images. In Computer Vision-ECCV 2008, pages 440-453. Springer, 2008.

[105] Daniele Palossi, Federico Tombari, Samuele Salti, Martino Ruggiero, Luigi Stefano, and Luca Benini. GPU-SHOT: Parallel Optimization for Real-time 3D Local Description. In Proceedings of the IEEE Conference on Computer Vision and Pattern Recognition Workshops, pages 584-591, 2013.

[106] S. Paschalakis et al. CDVS CE2: Local Descriptor Compression Proposal. In ISO/IEC JTC1/SC29/WG11/M25929.

[107] S. Paschalakis et al. Information Technology - Multimedia content descriptor interface Part 13: Compact Descriptors for Visual Search. in ISO/IEC DIS 15938-13.

[108] François Pomerleau, Francis Colas, Roland Siegwart, and Stéphane Magnenat. Comparing ICP Variants on Real-World Data Sets. Autonomous Robots, 34(3):133-148, 2013.

[109] Sai Manoj. Prakhya, Bingbing Liu, and Weisi. Lin. B-SHOT: A Binary Feature Descriptor for Fast and Efficient Keypoint Matching on 3D Point Clouds. In Intelligent Robots and Systems (IROS), 2015 IEEE/RSJ International Conference on, October 2015,.

[110] Sai Manoj. Prakhya, Bingbing Liu, and Weisi. Lin. On Creating Low Dimensional 3D Feature Descriptors with PCA. In Research Gate (Available Online), Dec 2016.

[111] Sai Manoj. Prakhya, Bingbing Liu, Weisi Lin, and Usman. Qayyum. Sparse Depth Odometry : 3D Keypoint based Pose Estimation from Dense Depth Data . In Robotics and Automation (ICRA), 2015 IEEE International Conference on, May 2015.

[112] S.M. Prakhya, Liu Bingbing, Yan Rui, and Weisi Lin. A Closed-form Estimate of 3D ICP Covariance. In Machine Vision Applications (MVA), 2015 14th IAPR International Conference on, pages 526-529, May 2015.

[113] Johann Prankl, Aitor Aldoma, Alexander Svejda, and Markus Vincze. Rgb-d object modelling for object recognition and tracking. In Intelligent Robots and Systems (IROS), 2015 IEEE/RSJ International Conference on, pages 96-103. IEEE, 2015.

[114] Rahul Raguram, Ondrej Chum, Marc Pollefeys, Jiri Matas, and Jan-Michael Frahm. USAC: A Universal Framework for Random Sample Consensus. IEEE transactions on pattern analysis and machine intelligence, 35(8):2022-2038, 2013. 
[115] Iaroslav Melekhov1 Juho Kannala1 Esa Rahtu. Image patch matching using convolutional descriptors with euclidean distance.

[116] Bharath Ramesh, Cheng Xiang, and Tong Heng Lee. Shape classification using invariant features and contextual information in the bag-of-words model. Pattern Recognition, 48(3):894-906, 2015.

[117] Shaoqing Ren, Kaiming He, Ross Girshick, and Jian Sun. Faster r-cnn: Towards realtime object detection with region proposal networks. In Advances in neural information processing systems, pages 91-99, 2015.

[118] Yuriy Reznik et al. An Algorithm for Quantization of Discrete Probability Distributions. In Data Compression Conference (DCC), 2011, pages 333-342. IEEE, 2011.

[119] Gernot Riegler, Ali Osman Ulusoys, and Andreas Geiger. Octnet: Learning deep 3d representations at high resolutions. arXiv preprint arXiv:1611.05009, 2016.

[120] Edward Rosten, Reid Porter, and Tom Drummond. Faster and Better: A Machine Learning Approach to Corner Detection. IEEE transactions on pattern analysis and machine intelligence, 32(1):105-119, 2010.

[121] Henry Roth and Marsette Vona. Moving Volume KinectFusion. In Proceedings of the British Machine Vision Conference, pages 112.1-112.11. BMVA Press, 2012.

[122] Ethan Rublee, Vincent Rabaud, Kurt Konolige, and Gary Bradski. ORB: An Efficient Alternative to SIFT or SURF. In 2011 International conference on computer vision, pages 2564-2571. IEEE, 2011.

[123] Szymon Rusinkiewicz and Marc Levoy. Efficient variants of the ICP algorithm. In 3-D Digital Imaging and Modeling, 2001. Proceedings. Third International Conference on, pages 145-152. IEEE, 2001.

[124] Radu Bogdan Rusu, Nico Blodow, and Michael Beetz. Fast Point Feature Histograms (FPFH) for 3D Registration. In Robotics and Automation, 2009. ICRA'09. IEEE International Conference on. IEEE, 2009.

[125] Radu Bogdan Rusu, Nico Blodow, Zoltan Csaba Marton, and Michael Beetz. Aligning Point Cloud Views using Persistent Feature Histograms. In Intelligent Robots and Systems, 2008. IROS 2008. IEEE/RSJ International Conference on, pages 3384-3391. IEEE, 2008. 
[126] R.B. Rusu and S. Cousins. 3D is here: Point Cloud Library (PCL). In Robotics and Automation (ICRA), 2011 IEEE International Conference on, pages 1-4, May 2011.

[127] Ruslan Salakhutdinov and Geoffrey Hinton. Semantic Hashing. International Journal of Approximate Reasoning, 50(7):969-978, 2009.

[128] Samuele Salti, Federico Tombari, and Luigi Di Stefano. SHOT: Unique Signatures of Histograms for Surface and Texture Description. Computer Vision and Image Understanding, 125:251-264, 2014.

[129] Michalis A Savelonas, Ioannis Pratikakis, and Konstantinos Sfikas. Partial 3D Object Retrieval combining Local Shape Descriptors with Global Fisher Vectors. 2015.

[130] Davide Scaramuzza and Friedrich Fraundorfer. Visual odometry [tutorial]. Robotics \& Automation Magazine, IEEE, 18(4):80-92, 2011.

[131] Edgar Simo-Serra, Eduard Trulls, Luis Ferraz, Iasonas Kokkinos, Pascal Fua, and Francesc Moreno-Noguer. Discriminative learning of deep convolutional feature point descriptors. In Proceedings of the IEEE International Conference on Computer Vision, pages 118-126, 2015.

[132] A. Sluzek. Contextual descriptors improving credibility of keypoint matching: Harrisaffine, hessian-affme and sift feasibility study. In 2014 13th International Conference on Control Automation Robotics Vision (ICARCV), pages 117-122, Dec 2014.

[133] Richard Socher, Brody Huval, Bharath Bath, Christopher D Manning, and Andrew Y Ng. Convolutional-recursive deep learning for 3d object classification. In Advances in Neural Information Processing Systems, pages 665-673, 2012.

[134] B. Steder, R.B. Rusu, K. Konolige, and W. Burgard. Point Feature Extraction on 3D Range Scans Taking into Account Object Boundaries. In Robotics and Automation (ICRA), 2011 IEEE International Conference on, pages 2601-2608, May 2011.

[135] F. Steinbruecker, J. Sturm, and D. Cremers. Real-Time Visual Odometry from Dense RGB-D Images. In Workshop on Live Dense Reconstruction with Moving Cameras at the Intl. Conf. on Computer Vision (ICCV), 2011.

[136] F. Steinbruecker, J. Sturm, and D. Cremers. Volumetric 3D Mapping in Real-Time on a CPU. In Int. Conf. on Robotics and Automation, Hongkong, China, 2014. 
[137] Jorg Stuckler and Sven Behnke. Integrating Depth and Color cues for Dense Multiresolution Scene Mapping using RGB-D Cameras. In IEEE International Conference on Multisensor Fusion and Integration for Intelligent Systems (MFI), pages 162-167, 2012.

[138] J. Sturm, N. Engelhard, F. Endres, W. Burgard, and D. Cremers. A Benchmark for the Evaluation of RGB-D SLAM Systems. In International Conference on Intelligent Robot Systems (IROS), 2012.

[139] Juergen Sturm, Erik Bylow, Fredrik Kahl, and Daniel Cremers. CopyMe3D: Scanning and Printing Persons in 3D. In Pattern Recognition, Lecture Notes in Computer Science, pages $405-414$.

[140] Jian Sun, Maks Ovsjanikov, and Leonidas Guibas. A Concise and Provably Informative Multi-Scale Signature Based on Heat Diffusion. Computer Graphics Forum, 28(5):1383-1392, 2009.

[141] Anh T. Tra, Weisi Lin, and Alex Kot. Dominant SIFT : A Novel Compact Descriptor. In Acoustics, Speech and Signal Processing (ICASSP), 2014 IEEE International Conference on, May 2015.

[142] Hedi Tabia, Hamid Laga, David Picard, and Philippe-Henri Gosselin. Covariance Descriptors for 3D Shape Matching and Retrieval. In Computer Vision and Pattern Recognition (CVPR), 2014 IEEE Conference on, pages 4185-4192. IEEE, 2014.

[143] Federico Tombari, Samuele Salti, and Luigi Di Stefano. Unique Shape Context for 3D Data Description. In Proceedings of the ACM Workshop on 3D Object Retrieval, 3DOR '10, pages 57-62, New York, NY, USA, 2010. ACM.

[144] Federico Tombari, Samuele Salti, and Luigi Di Stefano. Unique Signatures of Histograms for Local Surface Description. In Computer Vision-ECCV 2010, pages 356369. Springer, 2010.

[145] Federico Tombari, Samuele Salti, and Luigi Di Stefano. Performance Evaluation of 3D Keypoint Detectors. International Journal of Computer Vision, 102(1-3):198-220, 2013.

[146] Federico Tombari, Samuele Salti, and Luigi Di Stefano. A Combined Texture-Shape Descriptor for Enhanced 3D Feature Matching. In Image Processing (ICIP), 2011 18th IEEE International Conference on, pages 809-812. IEEE, 2011. 
[147] Federico Tombari, Samuele Salti, and Luigi Di Stefano. Performance Evaluation of 3D Keypoint Detectors. International Journal of Computer Vision, 102(1-3):198-220, 2013.

[148] Andrea Torsello, Emanuele Rodola, and Andrea Albarelli. Sampling Relevant Points for Surface Registration. In 3D Imaging, Modeling, Processing, Visualization and Transmission (3DIMPVT), 2011 International Conference on, pages 290-295. IEEE, 2011.

[149] Tomasz Trzcinski, Mario Christoudias, and Vincent Lepetit. Learning Image Descriptors with Boosting. Pattern Analysis and Machine Intelligence, IEEE Transactions on, 37(3):597-610, 2015.

[150] Ranjith Unnikrishnan and Martial Hebert. Multi-scale interest regions from unorganized point clouds. In Computer Vision and Pattern Recognition Workshops, 2008. CVPRW'08. IEEE Computer Society Conference on, pages 1-8. IEEE, 2008.

[151] Yannick Verdie, Kwang Moo Yi, Pascal Fua, and Vincent Lepetit. Tilde: A temporally invariant learned detector. In Computer Vision and Pattern Recognition (CVPR). IEEE, 2015.

[152] Dominic Zeng Wang and Ingmar Posner. Voting for Voting in Online Point Cloud Object Detection. Proceedings of the Robotics: Science and Systems, Rome, Italy, 1317, 2015.

[153] Shandong Wang, Lujin Gong, Hui Zhang, Yongjie Zhang, Haibing Ren, Seon-Min Rhee, and Hyong-Euk Lee. SDTP: A Robust Method for Interest Point Detection on 3D Range Images. volume 9025, pages 902500-902500-9, 2014.

[154] T. Whelan, M. Kaess, M.F. Fallon, H. Johannsson, J.J. Leonard, and J.B. McDonald. Kintinuous: Spatially Extended KinectFusion. In RSS Workshop on RGB-D: Advanced Reasoning with Depth Cameras, Sydney, Australia, Jul 2012.

[155] Thomas Whelan, Hordur Johannsson, Michael Kaess, John J Leonard, and John McDonald. Robust Real-time Visual Odometry for Dense RGB-D mapping. In IEEE Interantional Conference on Robotics and Automation, ICRA, 2013.

[156] Jiaolong Yang, Hongdong Li, Dylan Campbell, and Yunde Jia. Go-icp: A globally optimal solution to $3 \mathrm{~d}$ icp point-set registration. In IEEE Transactions on Pattern Analysis and Machine Intelligence (T-PAMI), 2016. 
[157] Xin Yang and Kwang-Ting Cheng. Local Difference Binary for Ultrafast and Distinctive Feature Description. Pattern Analysis and Machine Intelligence, IEEE Transactions on, 36(1):188-194, 2014.

[158] Kwang Moo Yi, Eduard Trulls, Vincent Lepetit, and Pascal Fua. Lift: Learned invariant feature transform. In European Conference on Computer Vision (ECCV), 2016.

[159] Sergey Zagoruyko and Nikos Komodakis. Learning to compare image patches via convolutional neural networks. In Proceedings of the IEEE Conference on Computer Vision and Pattern Recognition, pages 4353-4361, 2015.

[160] Andrei Zaharescu, Edmond Boyer, Kiran Varanasi, and Radu Horaud. Surface feature detection and description with applications to mesh matching. In Computer Vision and Pattern Recognition, 2009. CVPR 2009. IEEE Conference on, pages 373-380. IEEE, 2009.

[161] Andy Zeng, Shuran Song, Matthias Nießner, Matthew Fisher, and Jianxiong Xiao. 3dmatch: Learning the matching of local $3 \mathrm{~d}$ geometry in range scans. arXiv preprint arXiv:1603.08182, 2016.

[162] Yu Zhong. Intrinsic Shape Signatures: A Shape Descriptor for 3D Object Recognition. In Computer Vision Workshops (ICCV Workshops), 2009 IEEE 12th International Conference on, pages 689-696, Sept 2009.

[163] Wengang Zhou, Houqiang Li, Richang Hong, Yijuan Lu, and Qi Tian. BSIFT: Toward Data-Independent Codebook for Large Scale Image Search. Image Processing, IEEE Transactions on, 24(3):967-979, March 2015. 